\title{
Development of an Ammonia Reduction After-Treatment Systems for Stoichiometric Natural Gas Engines
}

Saroj Pradhan

Follow this and additional works at: https://researchrepository.wvu.edu/etd

\section{Recommended Citation}

Pradhan, Saroj, "Development of an Ammonia Reduction After-Treatment Systems for Stoichiometric Natural Gas Engines" (2017). Graduate Theses, Dissertations, and Problem Reports. 6447.

https://researchrepository.wvu.edu/etd/6447

This Dissertation is protected by copyright and/or related rights. It has been brought to you by the The Research Repository @ WVU with permission from the rights-holder(s). You are free to use this Dissertation in any way that is permitted by the copyright and related rights legislation that applies to your use. For other uses you must obtain permission from the rights-holder(s) directly, unless additional rights are indicated by a Creative Commons license in the record and/ or on the work itself. This Dissertation has been accepted for inclusion in WVU Graduate Theses, Dissertations, and Problem Reports collection by an authorized administrator of The Research Repository @ WVU.

For more information, please contact researchrepository@mail.wvu.edu. 


\title{
Development of an Ammonia Reduction After-treatment Systems for Stoichiometric Natural Gas Engines
}

\author{
Saroj Pradhan \\ Dissertation submitted to the \\ Benjamin M. Statler College of Engineering and Mineral Resources \\ at West Virginia University \\ in partial fulfillment of the requirements for the degree of \\ Doctor of Philosophy \\ in \\ Mechanical Engineering \\ Arvind Thiruvengadam, Ph.D., Chair \\ Marc C. Besch, Ph.D. \\ Mario Perhinschi, Ph.D. \\ Bharadwaj Shathiamoorthy, Ph.D. \\ Ross Ryskamp, Ph.D. \\ Department of Mechanical and Aerospace Engineering \\ Morgantown, West Virginia \\ 2017
}

Keywords: stoichiometric natural gas engines, three-way catalyst, SCR, ammonia emission, after-treatment modelling, artificial intelligence techniques, lean-burn spark ignited

Copyright 2017 Saroj Pradhan 


\section{ABSTRACT \\ Development of an Ammonia Reduction After-treatment Systems for Stoichiometric Natural Gas Engines}

\section{Saroj Pradhan}

Three-way catalyst (TWC) equipped stoichiometric natural gas vehicles have proven to be an effective alternative fuel strategy that shows significant low NOx emissions characteristics. However, recent studies have shown the TWC activity to contribute to elevated levels of tailpipe ammonia $\left(\mathrm{NH}_{3}\right)$ emissions. Although a non-regulated pollutant, ammonia is a potent pre-cursor to ambient secondary PM formation. Ammonia is an inevitable byproduct of fuel rich operation that results in lowest NOx slip through the TWC after-treatment system.

The main objective of the study is to develop a passive Ammonia Reduction Catalyst (passive-ARC) based $\mathrm{NH}_{3}$ reduction strategy that results in an overall reduction of ammonia as well as NOx emissions. The study investigated the characteristics of Fe-based and Cu-based zeolites SCR catalysts in storage and desorption of ammonia at high exhaust temperature conditions, that are typical of stoichiometric natural gas engines. Continuous measurements of NOx and $\mathrm{NH}_{3}$ before and after the SCR systems were conducted using a Fourier Transform Infrared Spectrometry (FTIR) gas analyzer. Results of the investigation showed that both, the Fe- and $\mathrm{Cu}$ zeolite SCRs adsorbed above $90 \%$ of TWC generated $\mathrm{NH}_{3}$ emissions below $350-$ $375^{\circ} \mathrm{C}$ SCR temperatures. Desorption or slipping of $\mathrm{NH}_{3}$ was observed at exhaust gas temperatures exceeding $400^{\circ} \mathrm{C}$. In terms of NOx conversions, Fe-zeolite showed efficiency between $50-80 \%$ above temperatures of $300-350^{\circ} \mathrm{C}$ while $\mathrm{Cu}$-zeolite performed well at lower SCR temperature from $250^{\circ} \mathrm{C}$ and above with a conversion efficiency of greater than $50 \%$.

In order to efficiently reduce both $\mathrm{NOx}$ and $\mathrm{NH}_{3}$ simultaneously over longer durations it was found that an engine-based air fuel ratio operation strategy for the passive-ARC system must be developed. To this extent, the study extended its objectives to develop an engine-based control strategy that results in stoichiometric ammonia production operation followed by brief lean operation to regenerate the saturated ammonia reduction catalyst using high NOx slip through TWC. The study presents comprehensive results of ammonia storage characteristics of SCRs pertaining to stoichiometric natural gas engine exhaust as well as an advanced engine control strategy approach to simultaneously reduce both $\mathrm{NOx}$ and $\mathrm{NH}_{3}$ using an alternating air -fuel ratio approach. 


\section{ACKNOWLEDGEMENT}

From the very first day my parents held my hands and dropped me off at kindergarten back in Kathmandu, to finally wrapping up the final chapters of academic career at $W V U$, this long journey far away from home wouldn't even have been possible without all their sacrifices, endless support and unconditional love. Thank you, dad and mom, for believing in me. Also, I would like to thank my loving sister, Srijana, for all your support and motivation.

I would like to express my extreme appreciation to my advisor, mentor, and friend Dr. Arvind. Your support and encouragement has been a tremendous guidance in this last 5 years of mine. And, our buddy Dr. Besch or Marc Sir!, truly a most humble, friendly, caring, and knowledgeable person I have ever met in my life. This CAFEE journey wouldn't have been the same without both of you. I have learned and experienced so much working and shadowing from you guys, and never would forget my first days of refuse-truck testing when I got the true flavor of LA.

I would like to thank rest of my committee: Dr. Perhinschi, for your insightful comments and your wonderful AI class which has helped me accomplish lot of task within this work. And, Dr. Ross and Dr. Bharadwaj, also my two good old friends for providing me guidance and support not only for this work but in other research work.

Furthermore, a special thanks to my buddies Beeko Berk and Paul Pragalath for all the support in this work, and in all the other projects we have tackled together. I am also grateful to Brad, Richard and Jason for helping me in the lab and guiding me towards the right tools necessary to complete this work on time. I am also thankful to all my work colleagues for helping me in the projects and giving me, five years of fun filled moments in CAFEE.

I also thank you Mridul Gautam for your guidance and inspirational speeches which has helped me a lot in growing my confidence, and I always aim to follow your footsteps. A very special thanks to Dan for all your support and motivation you gave us over the CAFEE years.

And finally, last but not the least, my special gratitude goes to all the people who has been a part of this journey and my life. I can't thank you all enough for the care, support and encouragement. 


\section{TABLE OF CONTENT}

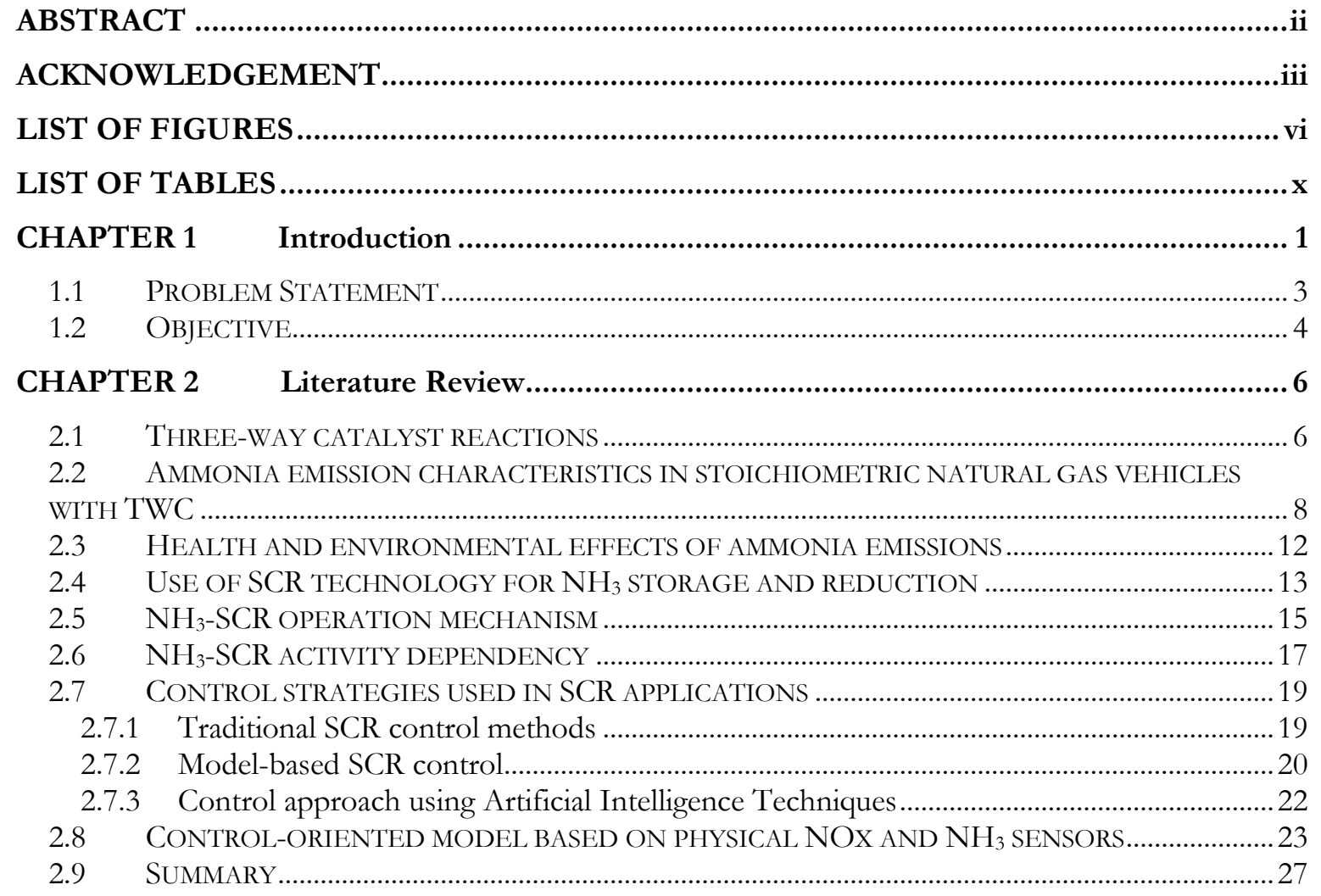

CHAPTER $3 \quad$ Experimental Setup …........................................................................... 29

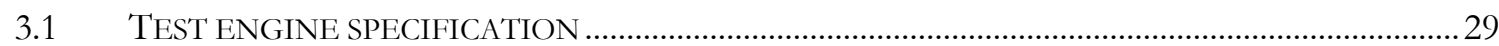

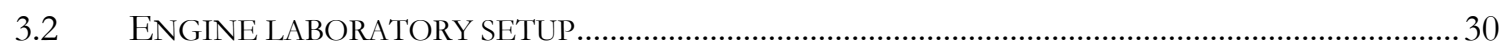

3.3 GASEOUS EMISSION MEASUREMENT SYSTEM USING FTIR ANALYZER ....................................31

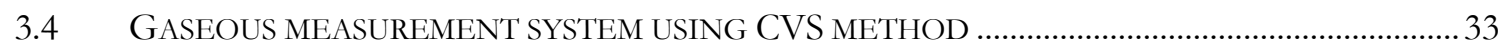

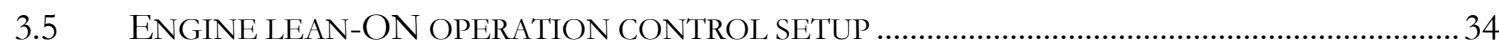

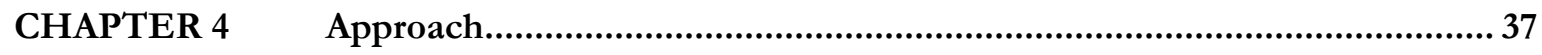

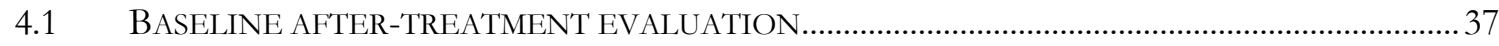

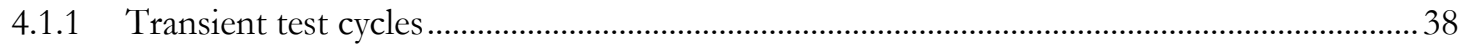

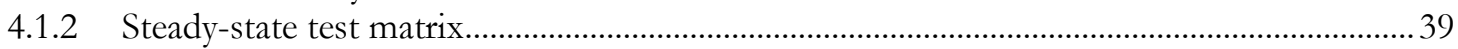

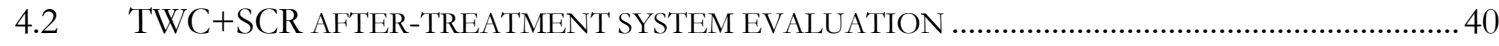

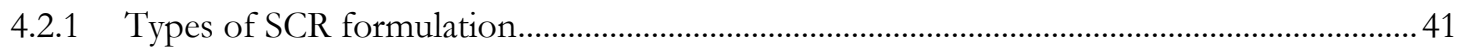

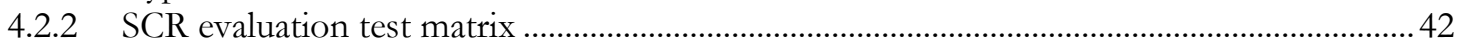

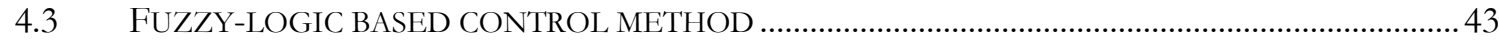

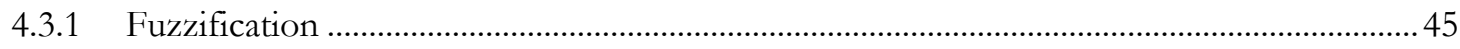

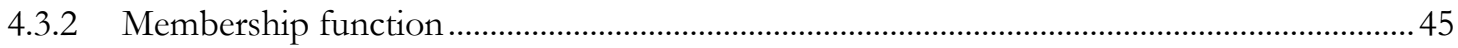

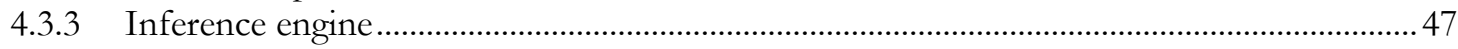

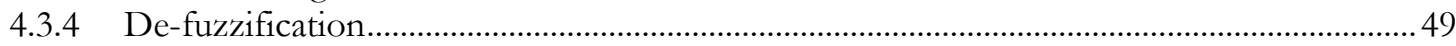

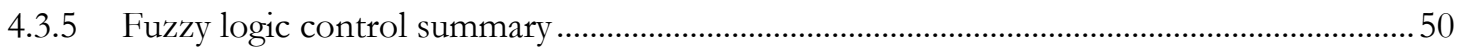

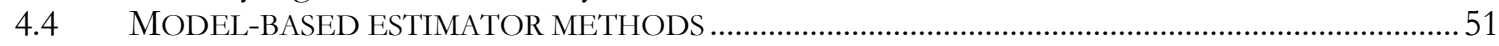

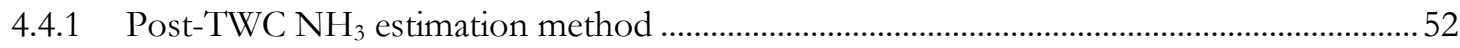

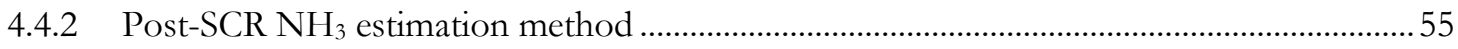

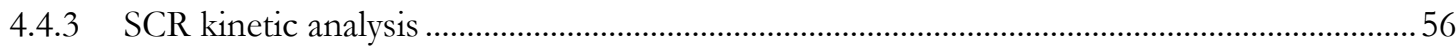




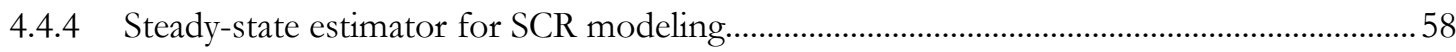

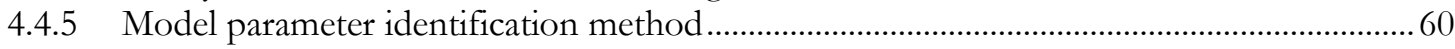

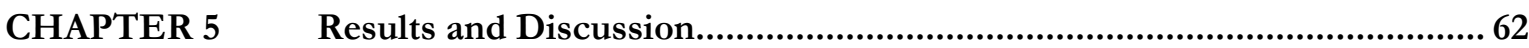

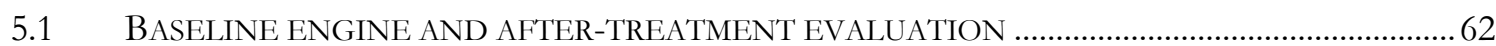

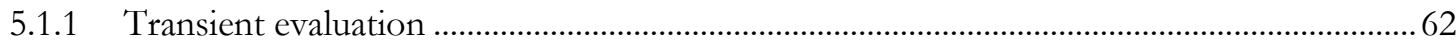

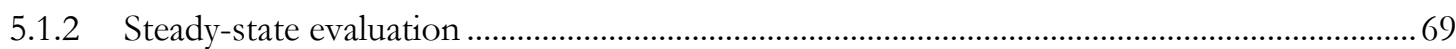

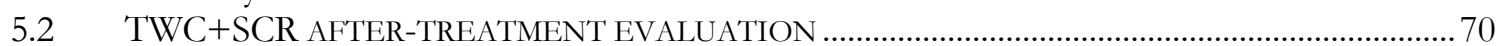

5.3 AMMONIA ADSORPTION/DESORPTION EVALUATION (SCR 4) …................................................. 77

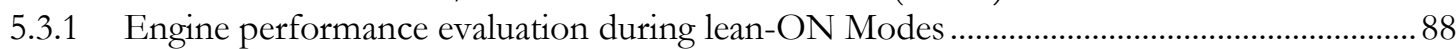

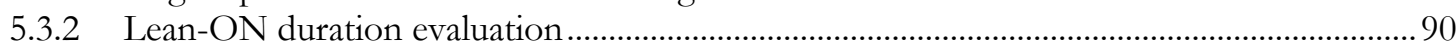

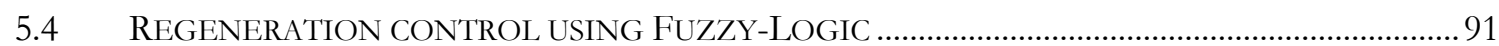

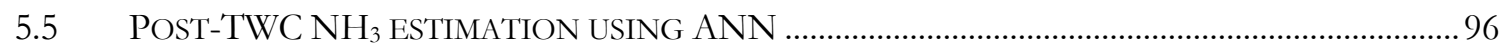

5.6 SCR MODEL CALIBRATED TO EXPERIMENTAL DATA …............................................................... 108

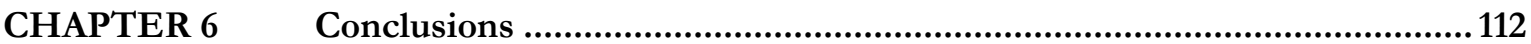

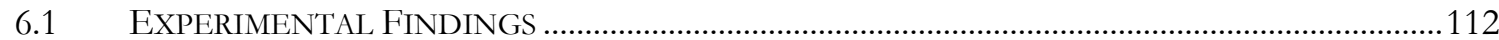

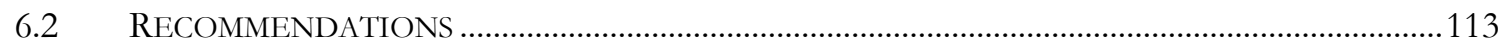

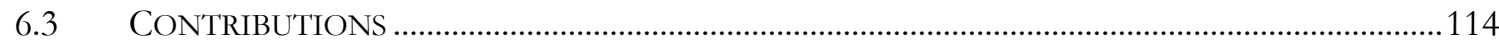

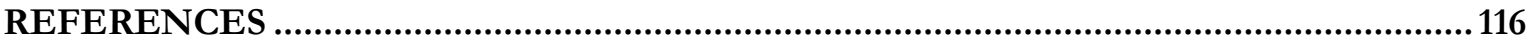

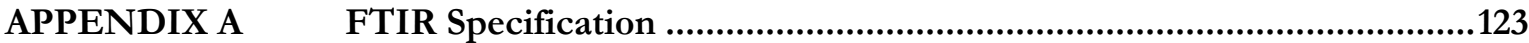

APPENDIX B $\quad$ Fuzzy Logic Controller Design and Program Code ..............................124 


\section{LIST OF FIGURES}

Figure 1. Effect of AFR sweep on $\mathrm{NH}_{3}$ formation over a TWC fir gasoline engine source (Li et al.

2010)

Figure 2. $\mathrm{NH}_{3}$ emission from a transit bus natural gas vehicle (Veh 4) over UDDS test

(Thiruvengadam et al. 2016)

Figure 3. $\mathrm{NOx}$ and $\mathrm{NH}_{3}$ emissions (g/bhp-hr) from five vocational natural gas vehicles

(Thiruvengadam et al. 2016)

Figure 4. Light-off curves corresponding to fresh versus aged TWC with different formulation

(González-velasco et al. 2000) ...

Figure 5. Ammonia storage capacity for two SCR formulation as a function of temperature (Eijnden et

al. 2009)

Figure 6. NOx conversion efficiency for vanadium-based and metal-exchanged zeolite-based SCR activity at varying temperatures under standard-SCR conditions (Kröcher 2007) ......................................17

Figure 7. Schematic of a NOx- $\mathrm{ZrO}_{2}$ sensor (Blanco-Rodriguez 2014) .....................................................24

Figure 8. Performance of $\mathrm{NOx}$ sensor $\mathrm{NH}_{3}$ cross-sensitivity factor using different error estimation

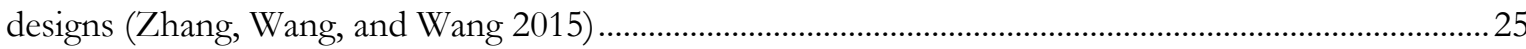

Figure 9. Schematic of $\mathrm{NH}_{3}$ sensor based on non-equilibrium electrochemical sensing principle (Moos

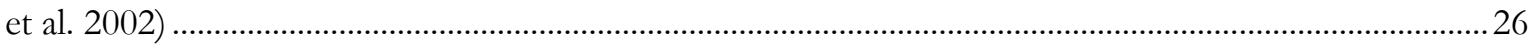

Figure 10. Baseline Cummins ISL-G test cell setup.................................................................................... 30

Figure 11. Cummins ISL-G (TWC+SCR) test cell setup ........................................................................ 31

Figure 12. Schematic diagram of an FTIR interferometer (Sanchonx 2017) ............................................. 32

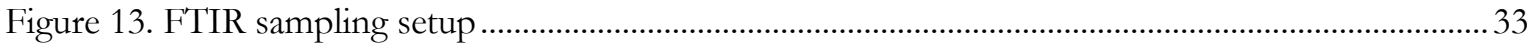

Figure 14. Schematic of dilute gaseous measurement system using CVS method......................................34

Figure 15. Manual lean-ON hardware control setup ……........................................................................... 35

Figure 16. Schematic of manual lean-ON control setup for intercepting on-board $\mathrm{O}_{2}$ sensor signal to

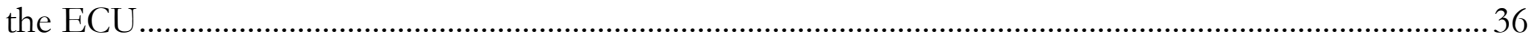

Figure 17. Schematic of the experimental setup for the baseline (engine + TWC) evaluation.................37

Figure 18. FTP normalized speed and torque profiles ................................................................................ 38

Figure 19. WHTC normalized speed and torque profiles ........................................................................... 38

Figure 20. Normalized distribution of engines operating points for the FTP and WHTC cycles ...........39

Figure 21. 20 engine operating modes for steady-state evaluation .......................................................... 40

Figure 22. Schematic of the experimental setup for the (engine + TWC + SCR) evaluation.................. 41

Figure 23. Transient bus real-world engine operation and 8-mode steady-state from clustering method

Figure 24. Flow-chart showing the fuzzy-logic based controller for controlling the passive-ARC

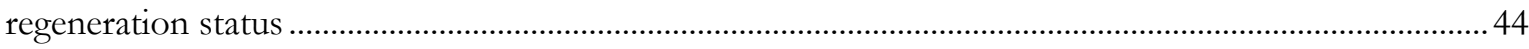

Figure 25. Membership function shapes for three input linguistic variables and its values......................46

Figure 26. Membership function for output variable.............................................................................. 47

Figure 27. 48 inference rule based matrix for fuzzy-logic output ............................................................. 48

Figure 28. De-fuzzification of the fuzzy-logic output using middle of maximum method...................... 49

Figure 29. Schematic of model-based $\mathrm{NH}_{3}$ estimation approach for the fuzzy-logic based controller.. 52 Figure 30. Example flow model architecture of ANN with seven inputs, three hidden layers and one

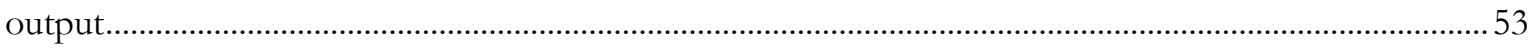

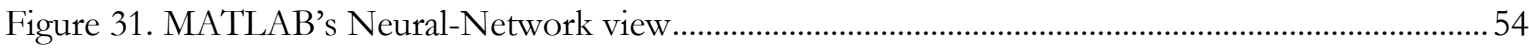


Figure 32. Schematic of ammonia storage distribution and CSTR model of SCR catalyst ......................58

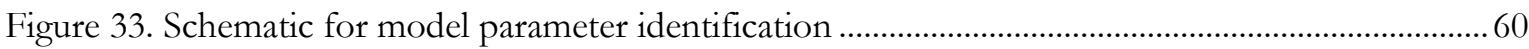

Figure 34. Averaged $\mathrm{NOx}, \mathrm{NH}_{3}, \mathrm{CO}$ and THC emission concentration before and after the TWC

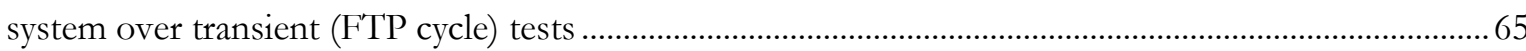

Figure 35. Averaged $\mathrm{NOx}, \mathrm{NH}_{3}, \mathrm{CO}$ and THC emission concentration before and after the TWC

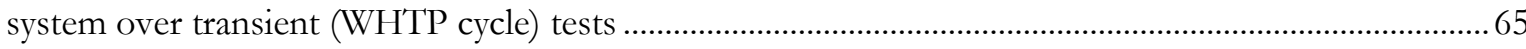

Figure 36. Engine transient operating (FTP-1 test) profiles showing engine speed (rpm), engine torque

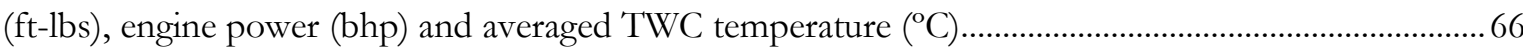

Figure 37. $\mathrm{NOx}$ and $\mathrm{NH}_{3}$ concentration before and after the TWC system over transient (FTP-2 test)

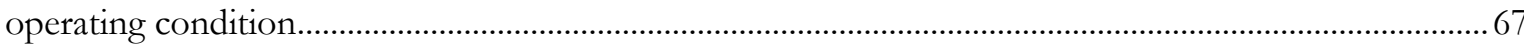

Figure 38. $\mathrm{NO}_{2}$ and $\mathrm{N}_{2} \mathrm{O}$ concentration before and after the TWC system over transient (FTP-1 test)

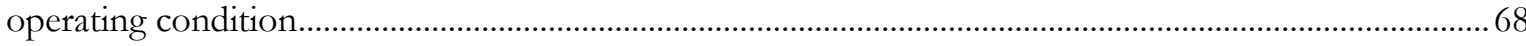

Figure 39. Averaged post-TWC NOx and $\mathrm{NH}_{3}$ concentration for temperature bins from FTP and WLTC cycles

Figure 40. Concentration counter plots from 20 steady-state mode tests represented under engine speed and torque region; (a) pre-TWC NOx (b) post-TWC NOx, (c) post-N2O and (d) post-TWC $\mathrm{NH}_{3}$

Figure 41. Profiles of NOx and $\mathrm{NH}_{3}$ concentration before and after the evaluated SCR over the FTP

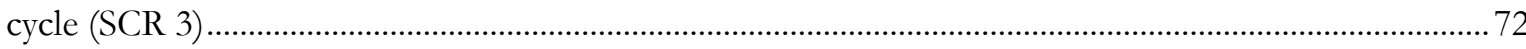

Figure 42. Cumulative profile of inlet $\mathrm{NH}_{3}, \mathrm{NH}_{3}$ storage and $\mathrm{NH}_{3}$ slippage from the evaluated SCR 3

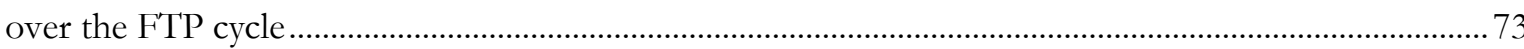

Figure 43: Comparison of averaged percent NOx conversion efficiency as a function of temperature

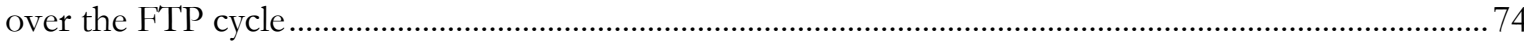

Figure 44: Comparison of averaged percent $\mathrm{NH}_{3}$ slip as a function of temperature over the FTP cycles

Figure 45. Average $\mathrm{NOx}$ conversion efficiency and $\mathrm{NH}_{3}$ slippage for all five $\mathrm{SCR}$ catalyst over the

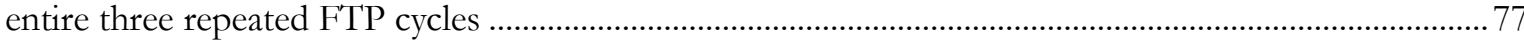

Figure 46: Mode 1 [engine speed $=751 \mathrm{rpm}$, engine torque $=247 \mathrm{ft}-\mathrm{lbs}$ ]; comparing pre-and-post

$\mathrm{NOx}$ and $\mathrm{NH}_{3}$ concentrations; includes lean-ON period (300-596 seconds) .......................................... 80

Figure 47: Mode 2 [engine speed $=851 \mathrm{rpm}$, engine torque $=84 \mathrm{ft}-\mathrm{lbs}$ ]; comparing pre-and-post NOx

and $\mathrm{NH}_{3}$ concentrations; includes lean-ON period (480-700 seconds)

Figure 48: Mode 3 [engine speed $=1249 \mathrm{rpm}$, engine torque $=316 \mathrm{ft}-\mathrm{lbs}$ ]; comparing pre-and-post

$\mathrm{NOx}$ and $\mathrm{NH}_{3}$ concentrations; includes two lean-ON periods (2040-2280 seconds \& 2970-3034

seconds)

Figure 49: Mode 4 [engine speed $=1296 \mathrm{rpm}$, engine torque $=37 \mathrm{ft}-\mathrm{lbs}$ ]; comparing pre-and-post

$\mathrm{NOx}$ and $\mathrm{NH}_{3}$ concentrations; includes lean-ON period (310-550 seconds)

Figure 50: Mode 5 [engine speed $=1480 \mathrm{rpm}$, engine torque $=597 \mathrm{ft}-1 \mathrm{bs}$ ]; comparing pre-and-post

$\mathrm{NOx}$ and $\mathrm{NH}_{3}$ concentrations; includes two lean-ON periods (65-122 seconds \& 774-1010 seconds) 85

Figure 51: Mode 6 [engine speed $=1521 \mathrm{rpm}$, engine torque $=162 \mathrm{ft}-\mathrm{lbs}]$; comparing pre-and-post

$\mathrm{NOx}$ and $\mathrm{NH}_{3}$ concentrations; includes two lean-ON periods (412-419 seconds \& 460-479).............. 86

Figure 52: Mode 7 [engine speed $=1575 \mathrm{rpm}$, engine torque $=337 \mathrm{ft}-\mathrm{lbs}$ ]; comparing pre-and-post

$\mathrm{NOx}$ and $\mathrm{NH}_{3}$ concentrations; includes two lean-ON periods (182-789 seconds \& 1245-1464 seconds) 
Figure 53: Mode 8 [engine speed $=1611 \mathrm{rpm}$, engine torque $=683 \mathrm{ft}-\mathrm{lbs}$ ]; comparing pre-and-post $\mathrm{NOx}$ and $\mathrm{NH}_{3}$ concentrations; includes three lean-ON periods (90-219 seconds, 577-752 seconds \& 1211-1362 seconds)

Figure 54. Comparison of brake specific fuel consumption between baseline operation versus lean-ON operation

Figure 55. Comparison of varying lean-ON periods (Mode 3)

Figure 56. Ammonia Slip (AS), Rate of Ammonia Slip (RAS), and NOx Slip (NOS) Membership Function .92

Figure 57. Fuzzy-logic controller response for steady-state Mode 8 (SCR 4).......................................... 94

Figure 58. Fuzzy-logic controller response for steady-state Mode 3 (SCR 4)........................................... 94

Figure 59. Fuzzy-logic controller response for transient FTP cycle (SCR 3) ............................................ 95

Figure 60. Transient FTP profile of the seven input variables .................................................................. 96

Figure 61. Results for measured versus estimated $\mathrm{NH}_{3}$ concentration using number of neurons and Levenberg-Marquardt training function .98

Figure 62. Comparison of measured and estimated $\mathrm{NH}_{3}$ concentration using different number of neurons and Bayesian Regularization training function

Figure 63: Comparison of MSE using EWMA filter with different filtering periods

101

Figure 64. Comparison of measured and estimated $\mathrm{NH}_{3}$ concertation applying the EWMA filter (Filter Period $=6)$ .101

Figure 65. Comparison of measured and estimated $\mathrm{NH}_{3}$ concentration; (Number of Inputs $=9$, Number of Neurons $=40$, Fitting Function $=$ Bayesian Regularization, EWMA with Period $=6) \ldots .102$ Figure 66. Comparison of number of moles of $\mathrm{NH}_{3}$ per catalyst volume (moles $/ \mathrm{m}^{3}$ ) calculated from actual versus estimated $\mathrm{NH}_{3}$ concentration. .103

Figure 67. Difference between actual and estimated number of moles of $\mathrm{NH}_{3}$ per catalyst volume...103 Figure 68. Comparison of actual versus estimated $\mathrm{NH}_{3}$ concertation using validation FTP Data Set 1

Figure 69 Comparison of actual versus estimated $\mathrm{NH}_{3}$ concertation using validation FTP Data Set 2

Figure 70. Comparison of actual versus estimated number of moles of $\mathrm{NH}_{3}$ per catalyst volume $\left(\mathrm{moles} / \mathrm{m}^{3}\right)$ for FTP Data Set 1

Figure 71. Comparison of actual versus estimated number of moles of $\mathrm{NH}_{3}$ per catalyst volume $\left(\mathrm{moles} / \mathrm{m}^{3}\right)$ for FTP Data Set 2 .....

Figure 72. Comparison of actual versus estimated number of moles of $\mathrm{NH}_{3}$ per catalyst volume $\left(\right.$ moles $\left./ \mathrm{m}^{3}\right)$ periods for FTP Data Set 2 using three different EWMA filtering 107 Figure 73. Difference between actual versus estimated number of moles of $\mathrm{NH}_{3}$ per catalyst volume $\left(\mathrm{moles} / \mathrm{m}^{3}\right)$ periods for FTP Data Set 2 using three different EWMA filtering ..... 107 Figure 74. Comparison of post-SCR NOx, post-SCR NH 3 and $\mathrm{NH}_{3}$ storage ratio predicted by 3 -state SCR model storage ratio for steady-state Mode 1 (baseline operation); Initial ammonia storage ratio = 0.15 109

Figure 75. Comparison of post-SCR NOx, post-SCR NH 3 and $\mathrm{NH}_{3}$ storage ratio predicted by 3-state SCR model for steady-state Mode 1 (baseline operation); Initial ammonia storage ratio $=0.7 ; \ldots \ldots \ldots . .109$ Figure 76. Comparison of post-SCR NOx, post-SCR $\mathrm{NH}_{3}$ and $\mathrm{NH}_{3}$ storage ratio predicted by 3 -state SCR model for steady-state Mode 1 (baseline operation); Initial ammonia storage ratio $=0.15 ; \Omega$ as identified parameter. .110

Figure 77. MATLAB's ${ }^{\circledR}$ 'Fuzzy-Logic Toolbox (FL Main Design Interface)..... .124 
Figure 78. MATLAB ${ }^{\circledR}$ 's Fuzzy-Logic Toolbox (FL Membership Function Design Interface) ..............124

Figure 79. MATLAB ${ }^{\circledR}$ 's Fuzzy-Logic Toolbox (FL Inference Rule Design Interface)............................125

Figure 80. MATLAB ${ }^{\circledR}$ 's Fuzzy-Logic Toolbox (FL Rule Viewer) ...........................................................125 


\section{LIST OF TABLES}

Table 1. Test Engine and After-treatment Specification ...........................................................................29

Table 2. List of five experimented SCR catalyst for ammonia reduction evaluation.................................. 41

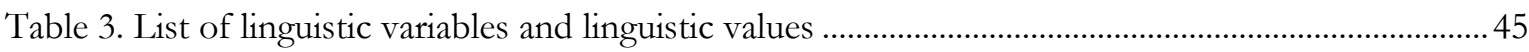

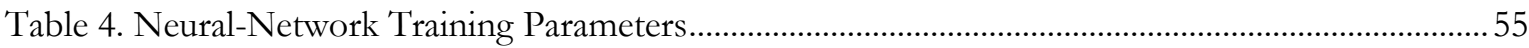

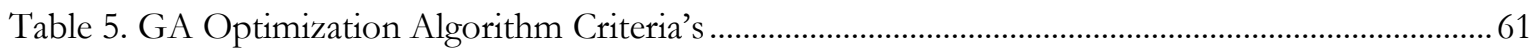

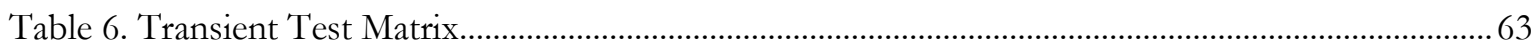

Table 7. Summary results from transient test cycles with baseline setup, CVS measured brake specific

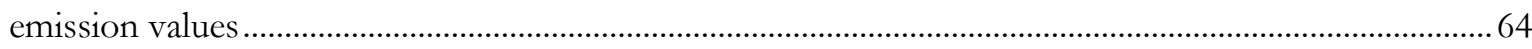

Table 8. Test matrix from the five SCR evaluation study ..................................................................... 71

Table 9. Steady-State mode test for SCR 4, engine and SCR temperature operating conditions ............ 78

Table 10. Summary comparison between baseline operation versus lean operation over eight steady-

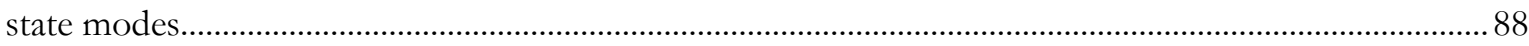

Table 11. Comparison of performance using different number of neurons and Levenberg-Marquardt

training function........................................................................................................................... 97

Table 12. Comparison of performance using different number of neurons and Bayesian Regularization

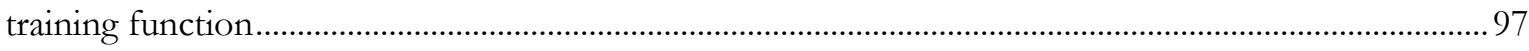

Table 13. Comparison of performance for four different number of neurons with Bayesian

Regularization training function and additional two first derivative input parameters ..........................100

Table 14. Reaction rate identified parameters in the three state SCR model............................................ 108

Table 15. Reaction rate identified parameters in the three state SCR model ............................................111

Table 16. FTIR Specification from Manufacturer...............................................................................123 


\section{CHAPTER 1 Introduction}

Considering today's in-use emission control devices that aid modern heavy-duty vehicles to comply with stringent USEPA emissions and $\mathrm{CO}_{2}$ greenhouse standards, natural gas fueled vehicles utilizing three-way catalyst (TWC) technology are known to provide better fuel consumption and lower tailpipe emission benefits when compared to the SCR equipped diesel counterparts (D. W. Stanton 2013). However, natural gas engines are precisely controlled to operate at a narrow stoichiometric combustion regime to effectively and simultaneously convert major toxic emissions such as nitrogen oxides (NOx), carbon monoxide (CO) and hydrocarbons (HC) in the TWC system. This has notably limited natural gas vehicles to operate the majority of their activity close to stoichiometric ratio, hence, leaving less room for fuel reduction improvements.

Efficiently converting the three major pollutants simultaneously from natural gas engine's exhaust stream over a TWC is commonly achieved by dithering the air-fuel ratio (AFR), a periodical switch between rich and lean combustion window operation (Defoort, Olsen, and Willson 2004). During the lean window, the three-way catalyst formulation is able to oxidize $\mathrm{HC}$ and $\mathrm{CO}$, and during the rich window favors redox reactions to reduce oxides of nitrogen (Shi et al. 2015). In addition to the standard reactions, studies have shown that under rich operating conditions, oxygen deficiency in the exhaust also promotes other two kinds of reactions known as water gas-shift and steam reforming reactions (Barbier and Duprez 1994). In such events, $\mathrm{CO}$ which is not oxidized due to lack of oxygen reacts with water vapor $\left(\mathrm{H}_{2} \mathrm{O}\right)$ to form $\mathrm{CO}_{2}$ and hydrogen $\left(\mathrm{H}_{2}\right)$. Further, under suitable conditions, the $\mathrm{H}_{2}$ molecules react with available $\mathrm{NO}$ and $\mathrm{CO}$ in the exhaust stream to form ammonia $\left(\mathrm{NH}_{3}\right)$, considered as a precursor emission (Suarez-Bertoa, Zardini, and Astorga 2014).

Gas-phase $\mathrm{NH}_{3}$ is also considered to be a critical atmospheric pollutant contributing to the formation of airborne particulate matter (Renner and Wolke, 2008). These secondary formed particles in the atmosphere have been studied by many researchers and institutions, and tend to show risk factors pertaining to human health (Suarez-Bertoa et al., 2014). As stated by International Agency for Research on Cancer, a part of the World Health Organization (WHO), particulate matter are the major constituents of outdoor air pollution and can cause cancer in humans (IARC, 2013). In addition to human health effects, secondary ammonia also impacts 
terrestrial vegetation (Krupa, 2003), and threatens ecosystem health by contributing to critical amount of nitrogen in remote ecosystems (Sun et al., 2016).

Numerous emission measurement conducted in laboratories, on-road in-use tests, roadside/tunnel studies by means of remote sensing methods and more have indicated high levels of tailpipe $\mathrm{NH}_{3}$ emissions from both gasoline and natural gas vehicles equipped with a TWC after-treatment system operating under stoichiometric combustion regime (Huai et al. 2003; Livingston, Rieger, and Winer 2009; Quiros et al. 2016). An emission inventory report published in August of 2000 by the South Coast Air Quality Management District estimated that mobile sources were the third largest source of $\mathrm{NH}_{3}$ emissions in the greater Los Angeles area, apart from agricultural and livestock waste sources, and accounted for approximately 18\% of the inventory. Similarly, a study conducted around the same time period showed that motor vehicles accounted for about $6 \%$ in North Carolina and 14\% in San Joaquin Valley (Battye Viney P., Roelle, Paul A. 2003). In Europe, a report published by the European Environment Agency's road transport sector showed an increase of 378\% in ammonia emission between 1990 and 2010.

Elevated levels of $\mathrm{NH}_{3}$ emission from natural gas vehicles could be attributed to several factors that promotes $\mathrm{NH}_{3}$ formation. Aging of the TWC over extensive vehicle operation has evidently shown to be one of the major contributor. Indeed, recent studies conducted by WVU have indicated high $\mathrm{NH}_{3}$ emission from such natural gas vehicles, and also reporting vehicle age attributing in varying levels of $\mathrm{NH}_{3}$ being emitted (Thiruvengadam et al. 2016). The deterioration of vehicle components and aging of the after-treatment system, as illustrated by the researchers, have shown to play a significant role in the operating characteristics of the TWC system. In specific to three-way catalyst performance, studies have shown that degradation of catalyst over time affects the chemical and/or thermal mechanisms of the catalyst material being used (Matam et al. 2012), and tend to influence oxidation storage capacity (Sabatini et al. 2016), emission conversion efficiencies, and light-off temperatures (Nagashima et al. 2000).

Although, a non-regulated pollutant in the US, increasing trend in $\mathrm{NH}_{3}$ emission from mobile sources, especially in urban areas and densely populated cities have received critical concerns over the years. In the heavy-duty transportation sector, natural gas-fueled vehicle population share a significantly lower market than the diesel-fueled vehicles. However, market studies have shown substantial penetration of natural gas-fueled vocational vehicles within urban areas, especially in the US (Delgado and Muncrief 2015). In vocational application captive 
fleets such as transit buses, refuse trucks, food/beverage delivery trucks and similar types, natural gas fueled vehicles are trending upward, attributed to the increasing number of fueling stations and vehicle procurement incentive programs at different federal and state levels. According to a conference paper presented by Stanton, a $11.9 \%$ average annual growth rate is projected between 2011 and 2040 for natural gas vehicles in the US (D. Stanton, Charlton, and Vajapeyazula 2013). As a result, such a trend could potentially increase vehicular $\mathrm{NH}_{3}$ emissions within major populated cities causing immediate as well as long term urban air quality degradation.

The above highlights pose a critical need to monitor $\mathrm{NH}_{3}$ emission from stoichiometric natural gas vehicles and develop strategies to capture or abate tailpipe $\mathrm{NH}_{3}$ emissions. In addition, $\mathrm{NH}_{3}$ reduction pathways can also lead to further reduction in $\mathrm{NOx}$ and GHG emissions, to potentially meet more stringent future regulations. The USEPA has initiated rulemakings for low-NOx emission standards, and if in force, the new standard would require all heavy-duty vehicles to meet $0.02 \mathrm{~g} / \mathrm{bhp}$-hr NOx regulation starting 2024 (USEPA 2016). This would force vehicle manufactures to reduce NOx emissions by almost $90 \%$ from the current standards, and would require significant advancement in emission control strategies and technologies.

\subsection{Problem Statement}

The study investigates the application of coupling an ammonia reduction technology to an existing stoichiometric natural gas after-treatment system downstream of the three-way catalyst (TWC) in order to reduce observed precursor- $\mathrm{NH}_{3}$ emissions. According to current industry practices, SCR systems have proven effective in reducing $\mathrm{NO}_{\mathrm{x}}$ emission by supplying of precisely controlled $\mathrm{NH}_{3}$, to aim selective reduction reactions over the catalyst. However, conversion performance of SCR system differs based on the catalyst formulation, where temperature and $\mathrm{NH}_{3}$ storage capability plays a critical role. The study considers investigating the characteristics and effects of different zeolite based SCR catalyst formulation on $\mathrm{NH}_{3}$ storage and $\mathrm{NO}_{\mathrm{x}}$ conversion efficiency operated over dynamic loading of a commercially used heavy-duty stoichiometric natural gas engine equipped with a TWC system.

A SCR system outlet of the TWC has mostly been explored for gasoline applications, as a lean-burn combustion strategy. This work however, demonstrates an active engine control regeneration approach to simultaneously reduce $\mathrm{NO}_{\mathrm{x}}$ and $\mathrm{NH}_{3}$, while at the same time obtain 
fuel reduction benefits. The regeneration process is initiated by precise control of air-fuel ratio, alternating between rich $\left(\mathrm{NH}_{3}\right.$ production mode) and lean $\left(\mathrm{NH}_{3}\right.$ regeneration mode) modes, where the ammonia absorbed during the normal stoichiometric operation in the SCR brick could be regenerated with the effect of high $\mathrm{NO}_{\mathrm{x}}$ slip through the TWC. The approach involves continuous monitoring of the SCR activity via utilizing additional $\mathrm{NH}_{3}$ and $\mathrm{NOx}$ sensors.

\subsection{Objective}

The global objective of this study is to demonstrate the applicability of an SCR system similar to one used in diesel application, to be used as an added Ammonia Reduction System (passive-ARC) that can store $\mathrm{NH}_{3}$ generated by the TWC system and then regenerated by brief, high NOx events when shifting the engine to lean-burn combustion. The theory behind the approach also leads to a pathway in further reducing overall NOx emissions and improve fuel consumption benefits for stoichiometric natural gas engines. The study is divided into two specific aims to achieve the global objective, and are provided as follows:

Specific Aim 1: Investigate $\mathrm{NOx}$ and $\mathrm{NH}_{3}$ reduction characteristics of different SCR catalyst over stoichiometric natural gas engine operating conditions. In summary, the specific aim is to identify a suitable system that performs well in ammonia storage capacity and NOx conversion characteristics over the wide operating conditions of a stoichiometric natural gas engine, and under the central hypothesis that a SCR catalyst is considered to be an adequate passive $\mathrm{NH}_{3}$ reduction system. The hypothesis was tested by performing SCR catalyst evaluation experiments over engine dynamometer procedure. Evaluation criteria included NOx conversion and $\mathrm{NH}_{3}$-slippage over different temperature ranges, emission constituent levels, and engine operating conditions. The SCR catalyst can then be used as a passive-ARC with a dual purpose of storing $\mathrm{NH}_{3}$ under specific conditions and then utilize the stored $\mathrm{NH}_{3}$ as a reducing agent in a strategic event of brief, lean-burn, high-NOx for ammonia regeneration (purge) which leads to the second specific aim of the study.

Specific Aim 2: Develop a SCR performance controlling technique consisting of a decision-making control feature, designed for actively regenerate ammonia stored within the catalyst by alternating between rich $\left(\mathrm{NH}_{3}\right.$ production) and lean $\left(\mathrm{NH}_{3}\right.$ regeneration using $\mathrm{NOx}$ reduction) modes with real-time feedback sensing based on either a physical sensor or from model-based estimation approach. Furthermore, the specific aim will also consider sensor 
reduction evaluation in conjunction to model-based control approach with SCR activity estimation methods focused on real-time, in-ECU application. 


\section{CHAPTER 2 Literature Review}

Three-way catalyst equipped stoichiometric natural gas vehicles have proven to be an effective alternative fuel strategy that has shown superior low NOx benefits in comparison to diesels equipped with SCR. However, in recent years, studies evaluating tailpipe emissions have shown the TWC activity to contribute to elevated levels of tailpipe ammonia emissions. Although a non-regulated pollutant, ammonia is a potent pre-cursor to ambient secondary PM formation. Ammonia is an inevitable catalytic byproduct of TWC during that results also corresponds to lowest $\mathrm{NOx}$ emissions. This chapter will focus on $\mathrm{NH}_{3}$ formation in the TWC, along with its known effects on the environment and human health. Also, an extensive review of available SCR technology utilizing $\mathrm{NH}_{3}$ will be presented. Additionally, a review will also be done on several types of after-treatment control strategies. In summary, the literature gathered for the study focuses on available research in the scientific and industry community that would potentially help in achieving the studies objectives.

\subsection{Three-way catalyst reactions}

The exhaust stream from a natural gas combustion primarily comprises of unburned hydrocarbons $(\mathrm{HC})$, carbon monoxide $(\mathrm{CO})$ and oxides of nitrogen $\left(\mathrm{NO}_{\mathrm{x}}\right)$. These emissions are effectively controlled with the help of commonly used three-way catalyst system, which uses combination of platinum $(\mathrm{Pt})$, rhodium $(\mathrm{Rh})$, and palladium $(\mathrm{Pd})$ as the active catalytic substances. Studies have shown that natural gas vehicles operated over stoichiometric combustion provide better performance over the older technology vehicles equipped with oxygen catalyst operated with lean-burn combustion in order to meet the more stringent 2010 $\mathrm{NO}_{\mathrm{x}}$ emission standards from the USEPA (Karavalakis et al. 2016). With the adequately designed three-way formulation, the catalyst promotes two kinds of reactions, oxidation reaction in the presence of oxygen to reduce $\mathrm{HC}$ and $\mathrm{CO}$ (given by Equation (1 and Equation (2), and reduction reaction to reduce $\mathrm{NO}_{\mathrm{x}}$ with presence of $\mathrm{CO}$ (given by Equation (3). 


$$
\begin{gathered}
\mathrm{CO}+1 / 2 \mathrm{O}_{2} \rightarrow \mathrm{CO}_{2} \\
\mathrm{CH}_{4}+2 \mathrm{O}_{2} \rightarrow \mathrm{CO}_{2}+\mathrm{H}_{2} \mathrm{O} \\
\mathrm{NO}+\mathrm{CO} \rightarrow 2 \mathrm{~N}_{2}+\mathrm{CO}_{2}
\end{gathered}
$$

Simultaneous reduction via the above-mentioned reaction of the three major gases in the natural gas exhaust is achieved via frequently varying the air-fuel ratio (AFR) of the engine combustion between rich and lean mixture, a commonly known strategy as fuel-dithering. This process have shown to be an effective approach in achieving greater efficiency in reducing NOx and $\mathrm{CO}$ during slightly rich operation and $\mathrm{HC}$ during slight lean operation (Defoort, Olsen, and Willson 2004). From a fuel-dithering optimization study for natural gas engine showed that the optimal dithering lambda-midpoint resides in slightly rich combustion, and precise control of frequency and amplitude of the fuel dithering tend to affect the overall performance characteristics of the TWC system (Shi et al. 2015).

In addition to the major chemical mechanism, two more reactions, water-gas shift reaction and steam reforming reactions have also known to commonly occur over the TWC system. Under suitable rich operating conditions of the engine when there is oxygen deficiency in the exhaust stream, favors unoxidized $\mathrm{CO}$ and $\mathrm{HC}$ to form $\mathrm{CO}_{2}$ and hydrogen $\left(\mathrm{H}_{2}\right)($ Barbier and Duprez 1994; Ohtsuka 2015), and expressed by Equation ((4) and ((5) below. Precursor $\mathrm{NH}_{3}$ is then generated over the catalyst in the presence of molecular $\mathrm{H}_{2}$ with available $\mathrm{NO}$ and CO (Nagashima et al. 2000), expressed mainly by the Equation (6) and (7).

$$
\begin{gathered}
\mathrm{CO}+\mathrm{H}_{2} \mathrm{O} \rightarrow \mathrm{CO}_{2}+\mathrm{H}_{2} \\
\mathrm{CH} 4+2 \mathrm{H}_{2} \mathrm{O} \rightarrow \mathrm{CO}_{2}+4 \mathrm{H}_{2} \\
2 \mathrm{NO}+5 \mathrm{H}_{2} \rightarrow 2 \mathrm{NH}_{3}+2 \mathrm{H}_{2} \mathrm{O} \\
2 \mathrm{NO}+2 \mathrm{CO}+3 \mathrm{H}_{2} \rightarrow 2 \mathrm{NH}_{3}+2 \mathrm{CO}_{2}
\end{gathered}
$$

From the above-mentioned reaction mechanisms, it shows that $\mathrm{NH}_{3}$ formation over the TWC is a chain effect of species and their kinetic behavior, and significantly impacted by varying 
AFR, either fuel rich combustion or oxygen rich combustion. Hence, different operating conditions lead to $\mathrm{NH}_{3}$ formation at different levels, which are reviewed in more details in the following sections.

\subsection{Ammonia emission characteristics in stoichiometric natural gas vehicles with TWC}

Ammonia formation mechanism have not been well studied in stoichiometrically operated heavy-duty natural gas engines equipped with a TWC system, however there have been multiple studies evidently showing high ammonia emission levels from such engine configuration and similar TWC system used in gasoline applications. These studies have shown that the characteristics in ammonia production is significantly dominated by engine operating at different AFR regime mainly to meet the required load demands and hence, directly affecting the amount of $\mathrm{NH}_{3}$ being produced over the catalyst (Defoort, Olsen, and Willson 2004). Figure 1 shows an example of how AFR impacts the dynamics of different species along with $\mathrm{NH}_{3}$ emission observed after a TWC operated in a gasoline engine application. According to the chart, the highest levels of TWC out $\mathrm{NH}_{3}$ occurs close to stoichiometric region in a typical gasoline engine exhaust.

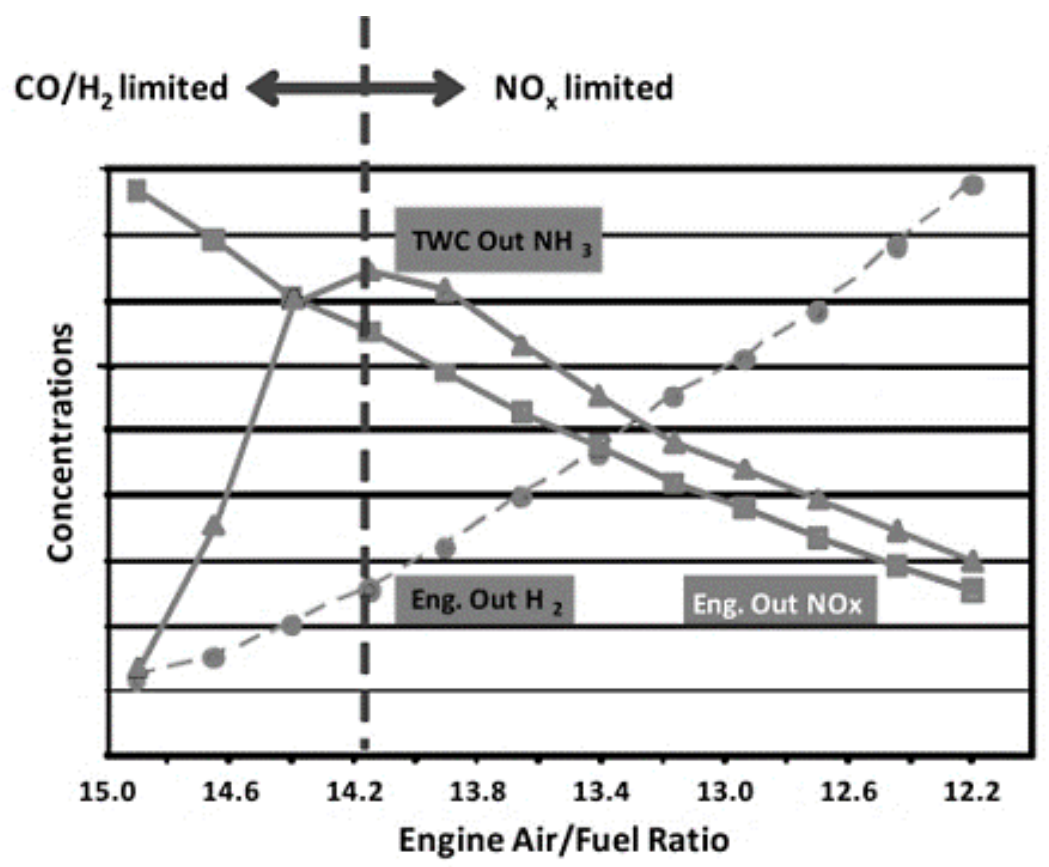

Figure 1. Effect of AFR sweep on $\mathrm{NH}_{3}$ formation over a TWC fir gasoline engine source (Li et al. 2010) 
From the point of power demand during on-road activity, driving behavior have shown as an influenceable factor in higher ammonia emissions seen from natural gas and gasoline sourced engines. Detailed emission measurement studies have shown that aggressive driving patterns or hard acceleration events result in high $\mathrm{NH}_{3}$ peaks in the exhaust (Huai et al. 2003). Sudden power demand with high acceleration corresponds to have richer AFR indicating sharper excursions in $\mathrm{NH}_{3}$ produced after the TWC. Recent studies conducted by WVU, also tend to evidently report such cycle trends in peak $\mathrm{NH}_{3}$ emissions (Thiruvengadam et al. 2016). The study measured $\mathrm{NH}_{3}$ concentration using Fourier Transform Infrared Spectroscopy (FTIR) analyzer from various natural gas transit buses and the study reported elevated levels of $\mathrm{NH}_{3}$ concentration, as high as $800 \mathrm{ppm}$, mainly produced during acceleration events as depicted in the Figure $2\left(\mathrm{NH}_{3}\right.$ formation observed only when the vehicle is in motion). In addition, studies have also shown high levels of $\mathrm{NH}_{3}$ emissions due to significant change in road grade, in instances where $\mathrm{NH}_{3}$ emission factors almost doubled when the road grade was increased from 0 to $7 \%$ (Sun et al. 2016).

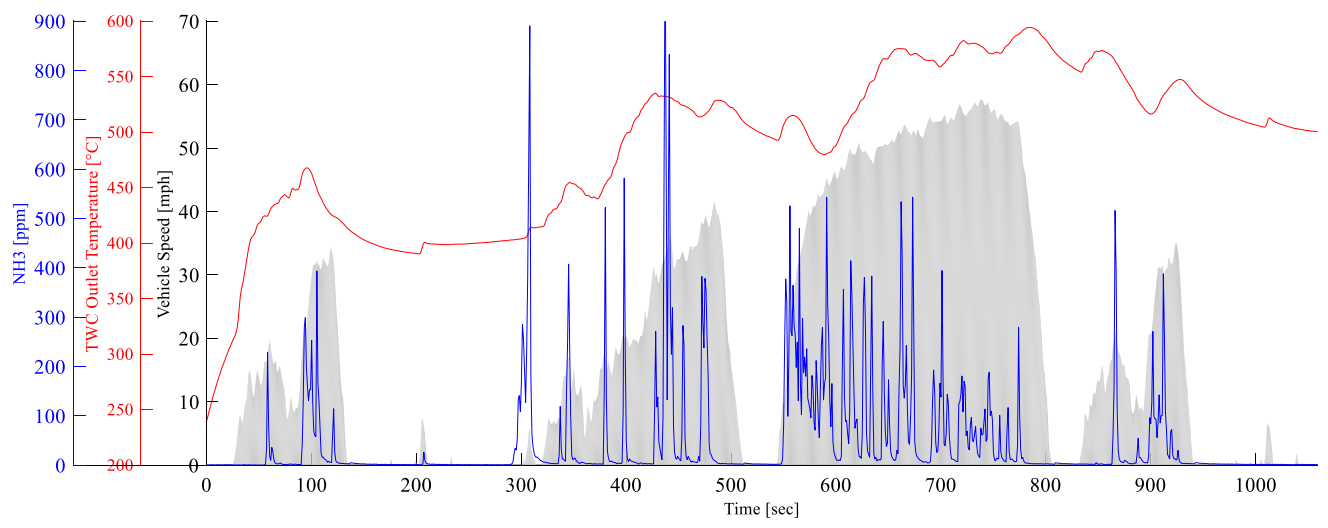

Figure 2. $\mathrm{NH}_{3}$ emission from a transit bus natural gas vehicle (Veh 4) over UDDS test

(Thiruvengadam et al. 2016)

In extension to the aforementioned WVU study that also tested similar natural gas vehicles having different accumulated vehicle mileage evidently showed higher brake-specific $\mathrm{NH}_{3}$ emissions, indicating vehicle age as a crucial factor. Figure 3 obtained from the study presents brake specific $\mathrm{NOx}$ and $\mathrm{NH}_{3}$ emissions for five different natural gas vehicles with two specific types of vocational application (Thiruvengadam et al. 2016). All five vehicles equipped with TWC system as the after-treatment technology were tested on a heavy-duty chassis 
dynamometer over the Urban Dynamometer Driving Schedule (UDDS) cycle. Results from the study highlights the fifth vehicle (Veh 5 as referred in the Figure 3) having the highest accumulated mileage, emitted the highest amount of $\mathrm{NH}_{3}$ emissions when compared to the other four vehicles. The brake-specific NOx results obtained from this vehicle also appeared to exceed the $0.2 \mathrm{~g} / \mathrm{bhp}-\mathrm{hr}$ USEPA 2010 NOx regulation levels for heavy-duty vehicle which was still considered to be below its useful life standards (USEPA 2011). The chart also shows result obtained from the fifth vehicle tested over real-world driving conditions which produced similar amounts of $\mathrm{NH}_{3}$ emission than the same vehicle tested over the chassis cycle but exhibit lower than $0.2 \mathrm{~g} / \mathrm{bhp}-\mathrm{hr}$ of NOx level.

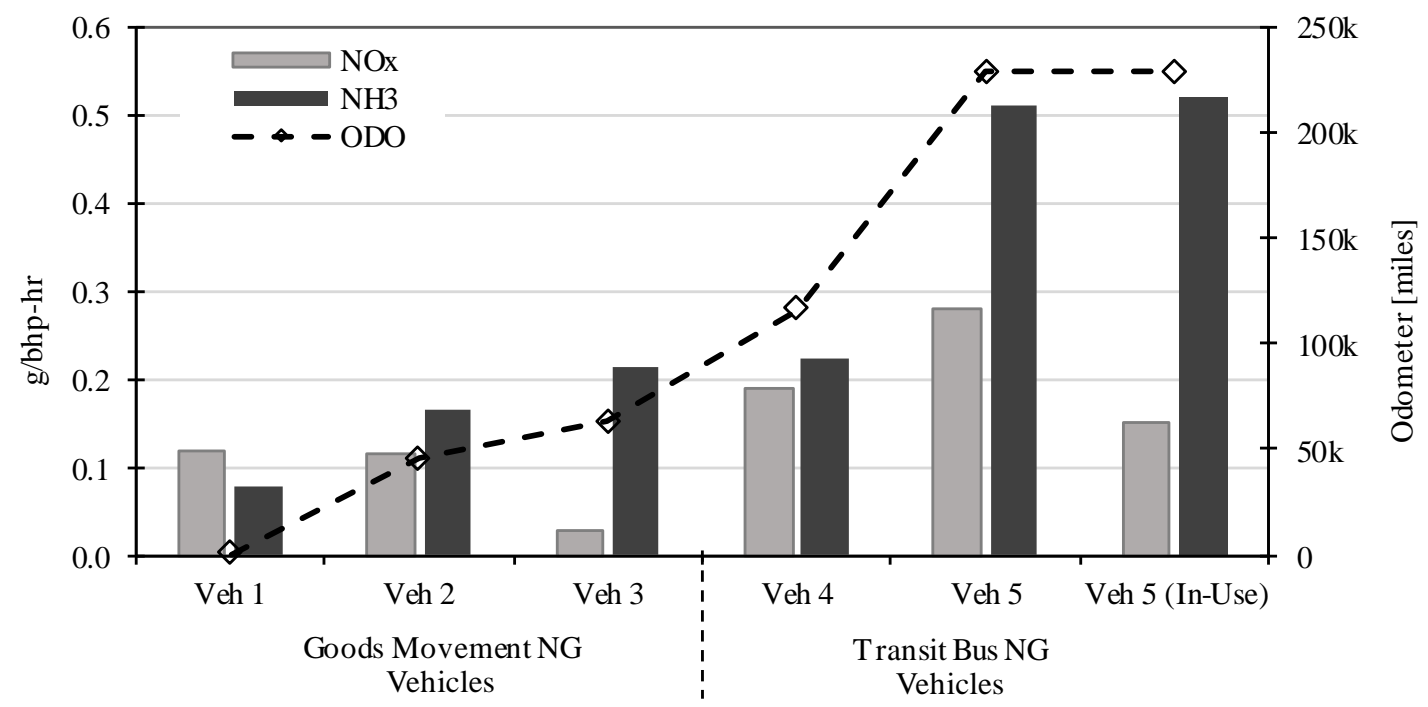

Figure 3. NOx and $\mathrm{NH}_{3}$ emissions (g/bhp-hr) from five vocational natural gas vehicles (Thiruvengadam et al. 2016)

Thiruvengadam et al. indicated degradation of emission control, such as the aging of an on-board feedback oxygen sensor could potentially cause sluggish response in the actual oxygen measurement, which in return affects the fuel dithering. And, this imbalance in engine operation control of the air-fuel ratio for the optimum TWC performance could lead to variability in tailpipe $\mathrm{NH}_{3}$ emissions (Thiruvengadam et al. 2016). A study by Wang et. al specifically studied the impact of oxygen sensor degradation on air-fuel ratio and emissions, and reported that a small shift in the signal from the oxygen sensor, for example, weather its upward, downward or even a slight delay in the sensor response, would deviate the conversion of the HC, $\mathrm{CO}$ and NOx to either increase or decrease (Dongliang Wang et al. 2011). 
Additionally, extensive experimental studies have also shown degradation of TWC due to catalyst aging, and are typically caused under chemical and/or thermal process of the TWC over repeated use in time, affecting the overall performance of the catalyst. Matam et al. studied the thermal and chemical aging in a model TWC with $\mathrm{Pd} / \mathrm{Al}_{2} \mathrm{O}_{3}$ for compressed natural gas vehicles exhaust and results showed that thermal aging leads to simply physical modification such as decreased surface area and dispersion of the catalyst material (Matam et al. 2012). The authors also highlights that physical modification clogs support pores along fouling of Pd nanoparticles. On the other hand, chemical aging was shown to be more detrimental than thermal aging for TWC performance, drastically decreasing the efficiency. The performance activity for a TWC to start converting $\mathrm{CO}, \mathrm{HC}$ and $\mathrm{NOx}$ above a certain efficiency greatly depends upon the light-off temperatures of catalyst material used, and such effect has been widely researched. For example, from a thermal aging study for a typical (Pd-Pt-Rh) combination based TWC, significant differences were observed in performance between fresh and aged catalyst (González-velasco et al. 2000). In this study it was shown that the light-off temperatures tend to shift towards a higher temperature with an aged catalyst and also considerably degrades performance in NOx conversion, as referred to the bottom right chart in Figure 4.

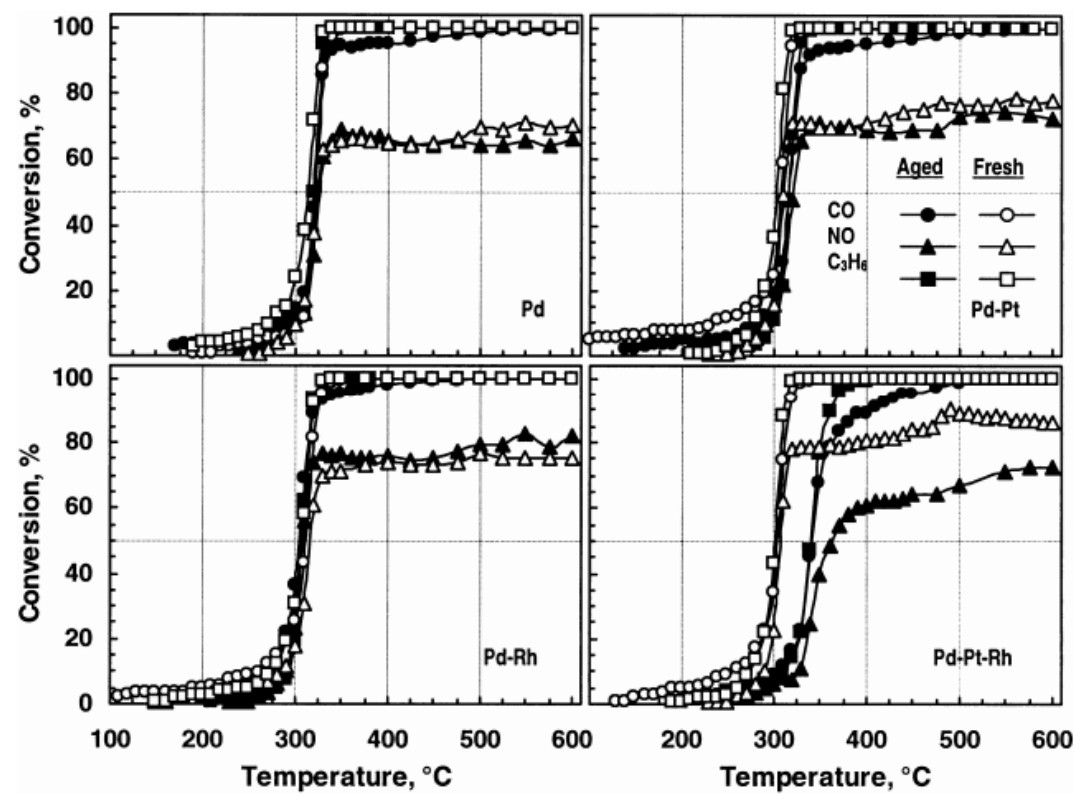

Figure 4. Light-off curves corresponding to fresh versus aged TWC with different formulation (González-velasco et al. 2000) 
Similarly, Sanatini et al. characterized the effects of aging on TWC, and reported aging substantially affects the oxygen storage dynamics of the catalyst and hence, affects the overall performance and formation of high $\mathrm{NH}_{3}$ emissions (Sabatini et al. 2016). The study mentions the importance of monitoring the oxygen concentration after the TWC to better understand the oxygen storage dynamics and proposes using a post lambda sensor as a good aging indicator. In order to better optimize oxygen storage capacity of the catalyst, loading interaction between Pt$\mathrm{Rh}$ and Pd-Rh in the TWC have contributed to be a major factor in achieving high performance even after extensive thermal aging (Nagashima et al. 2000). In addition to formulation loading, optimization of the substrate material such as alumina and Ce, also helps in compensating the high mobility of $\mathrm{Pt}$ at the high aging temperatures. Effects of Sulfur poising have also been studied for TWC activity which shown the degrade the TWC performance. Ohtsuka et. al examined Pt-Rh/ $\mathrm{CeO}_{2}-\mathrm{Al}_{2} \mathrm{O}_{3}$ TWC aged with $\mathrm{SO}_{2}$ under $400-500^{\circ} \mathrm{C}$ temperatures operating conditions, and presented results indicating significant decrease in performance activity, especially $\mathrm{CH}_{4}$ conversion (Ohtsuka 2015).

\subsection{Health and environmental effects of ammonia emissions}

Gas-phase ammonia has shown to contribute to the formation of airborne particulate matter (Renner and Wolke 2008). Research in this area has indicated that ammonia in ambient air neutralizes nitric and sulfuric acid to form ammonia nitrate $\left(\mathrm{NH}_{4} \mathrm{NO}_{3}\right)$ and sulfate $\left(\mathrm{NH}_{4}\right)_{2} \mathrm{SO}_{4}$, a two important components contributing to airborne fine particles or PM2.5 (Tengyu Liu Boguang Wang, Xiang Ding, Wei Deng, Sujun Lü, Yanli Zhang 2014). These secondary formed particulate matter in the atmosphere have been examined by many researchers and institutions, and tend to show risk factors pertaining to human health (SuarezBertoa, Zardini, and Astorga 2014). As has been stated by International Agency for Research on Cancer, a part of the World Health Organization (WHO), particulate matter are the major constituents of outdoor air pollution and can cause cancer in humans (IARC 2013). In addition to human health effects, secondary ammonia also impact terrestrial vegetation (Krupa 2003), and threatens ecosystem health by contributing to critical amounts of nitrogen in remote ecosystems (Sun et al. 2016).

Although studies have shown that exposure to ammonia at environmental concertation is unlikely to have direct adverse effects on human health, however, exposure to high concentration could cause irritation of the eyes, nose and throat as well as burning of the skin 
from direct contact. Ammonia, even at low concentrations, when released into air gives unpleasant odor, but most notably harm vegetation, particularly at high concentrations (Krupa 2003). On the other hand, ammonia in water bodies causes more serious harm due to the toxicity to aquatic organisms.

\subsection{Use of SCR technology for $\mathrm{NH}_{3}$ storage and reduction}

The use of SCR catalyst technologies have widely been investigated and proven to be an effective technology in removing NOx emissions from modern automotive vehicles equipped with compression ignited diesel engines (Tennison, Lambert, and Levin 2004; Johnson 2009; D. W. Stanton 2013; Nova Enrico 2014; Delgado and Muncrief 2015; Franco S. Francisco; German, John; Mock, Peter 2014). As widely adopted technology to reduce majority of the engine-out NOx emissions, large numbers of heavy-duty vehicle manufactures have also utilized engine-based control strategies in addition with the SCR system (D. Stanton, Charlton, and Vajapeyazula 2013). Engine-based strategy such as the exhaust gas recirculation (EGR) technology has provided manufactures in further meeting the stringent $\mathrm{NO}_{\mathrm{x}}$ emission standards.

Several studies have also demonstrated coupled after-treatment configurations with use of a SCR system downstream of the TWC, typically in spark ignited direct gasoline engines to achieve lean-burn fuel consumption benefits with NOx emission reductions (V. Y. Prikhodko et al. 2016; Li et al. 2010; Theis, Kim, and Cavataio 2015; Guralp et al. 2011). Researchers Guralp et al. demonstrated using a passive ammonia SCR system on an underfloor (U/F) SCR for storage of ammonia which is produced by the close-coupled TWC systems (Guralp et al. 2011). The study looked into oxygen tolerance and temperature requirements for the added SCR under stoichiometric conditions using a lab reactor experiment, and found that oxygen concentration was an important factor in order to get efficient NOx reduction over the catalyst. Based on the findings that NOx reduction efficiency was very low in the absence of oxygen, researchers experimented rich pulses operation where the SCR catalyst was able to store the ammonia produced on the close-coupled TWC and during lean pulse, NO was reduced by ammonia-SCR reaction over SCR catalyst. The study was also looked with use of combining with Deceleration Fuel Cut-Off (DFCO) and lean-idle (LI) operation strategies with the passive-SCR integration for fuel consumption improvement. The result indicated that in urban section of the FTP fuel consumption improved by $8.9 \%$ and additional improvement with a total of $11.1 \%$ when leanidle strategy also enabled. The work also highlights the benefits of using passive-SCR for 
stoichiometric application where the TWC can benefit from PGM loading requirements, performance improvement under varying operating strategies, increasing flexibility of air-fuel control and simplicity in the control techniques.

Study conducted by Prikhodko et al. demonstrated a 2.0L lean burn gasoline direct injection (GDI) engine utilizing a TWC system as an onboard $\mathrm{NH}_{3}$ producer, acquired during periodic rich engine operation and subsequently storing $\mathrm{NH}_{3}$ over a SCR (Cu-based) catalyst (V. Y. Prikhodko et al. 2016). The stored $\mathrm{NH}_{3}$ in the catalyst brick was then allowed to react with brief high levels of unreacted NOx slipped through the TWC during lean combustion operation. The study presented results of NOx conversion greater than $99 \%$ with the added passive SCR system and fuel consumption benefit ranging between (6-11\%) under certain engine condition when compared with its baseline stoichiometric operation. On the other hand, Prikhodko et al also studied a hybrid LNT+SCR system used to control NOx from a light-duty diesel engine with in-cylinder regeneration controls (J. P. and V. Prikhodko 2009). The results from the study showed that the $\mathrm{NH}_{3}$ formation increased strongly with extended regeneration of the LNT and cycling he control to lean-rich parameters enables controlling the ratio of NOx reduction between LNT and SCR catalysts. Additionally, the stored $\mathrm{NH}_{3}$ after multiple leanrich cycles can enable continued NOx reduction by the SCR the lean-rich period stops, assisting in better optimization during transient operation.

General Motors (GM) have published few different papers demonstrating passive ammonia-SCR system (PASS) indicating a potential low cost, simple and urea-free-system to enable the implementation of lean-burn gasoline engines ( $\mathrm{Li}$ et al., 2010, Kim, Perry, Viola, Li, \& Narayanaswamy, 2011). In the paper published by Li et al. showed NOx conversion efficiency greater than 85\% with passive SCR system for a 2.2L GDI engine. The experiments were evaluated with two kinds of zeolite-based SCRs, Cu- and Fe- tested over New European Driving Cycles (NEDC). On the other hand, the study published by Kim et al., indicated that the PASS needs to be adequately optimized for the PGM loading and oxygen storage control for $\mathrm{HC} / \mathrm{CO}$ control during both stoichiometric and rich operations. Some of the key parameters the authors mentions are the TWC wash-coat formulation that maximizes the efficiency of ammonia generation under mildly rich operating conditions, SCR formulation that maximizes $\mathrm{NH}_{3}$ storage and thermal stability at temperature greater than $400^{\circ} \mathrm{C}$. Comparison on ammonia yield and response time of a TWC and $\mathrm{Pd} / \mathrm{Al}_{2} \mathrm{O}_{3}$ catalyst combined with a passive SCR catalyst under dynamic engine operating conditions has also been investigated. It was observed that the yield 
of ammonia under rich condition was able to be maximized by using minimal valve overlap and an advanced spark timing, and additionally, use of low lambda values reduced the oxygen content of the exhaust gas, raising the $\mathrm{H}_{2}$ content resulting in increasing trend of the rate of $\mathrm{NO}$ conversion into ammonia. A study performed by Ford Motor Company also demonstrated a TWC but combining a lean-NOX trap (LNT) and a SCR catalyst for lean NOx control (Theis, Kim, and Cavataio 2015). The study showed equivalent $\mathrm{NOx}$ conversion and lower $\mathrm{NH}_{3}$ emissions, however, the approach resulted in higher fuel consumption due to LNT purge requirement and/or to generate $\mathrm{NH}_{3}$ for the SCR as compared to TWC+SCR only system. Additionally, the study also examined effect of sulfur poisoning on the combine configuration, and indicates that the SCR catalyst does not significantly get affected by sulfur poisoning, but the NH3 production and steam reforming capability of the TWC significantly degrades by sulfur during continuous operations at temperature near $500^{\circ} \mathrm{C}$. The authors also indicate that the closed coupled configuration would tend to remain in desulphated state with events caused from hot rich conditions during cold start, accelerations, and high load operations.

\section{5 $\mathrm{NH}_{3}-\mathrm{SCR}$ operation mechanism}

The basis of SCR application in diesel exhaust and, the main chemical reaction that occur over the SCR catalyst are provided by three main mechanisms. The first reaction provided by Equation (8), interpreted as "standard-SCR" (Koebel, Elsener, and Madia 2001), between $\mathrm{NH}_{3}, \mathrm{NO}$ and $\mathrm{O}_{2}$, where four mole of $\mathrm{NH}_{3}$ reacts with four mole of $\mathrm{NO}$ and one mole of $\mathrm{O}_{2}$ to produce $\mathrm{N}_{2}$ and $\mathrm{H}_{2} \mathrm{O}$. However, due to low availability of oxygen present in a typical stochiometric operated natural engine's exhaust, the reaction would occur at much slower rate, and potentially hinder $\mathrm{NO}$ to $\mathrm{NH} 3$ selective reduction reaction.

$$
4 \mathrm{NH}_{3}+4 \mathrm{NO}+\mathrm{O}_{2} \rightarrow 4 \mathrm{~N}_{2}+6 \mathrm{H}_{2} \mathrm{O}
$$

Subsequently, a faster known reaction (Bosch and Janssen 1988) between the reducing agent $\mathrm{NH}_{3}$, and mixture of an even ration of 1:1 between $\mathrm{NO}_{2}$ and $\mathrm{NO}$ is given by Equation (9). This reaction is also recognized as "fast-SCR" reaction.

$$
2 \mathrm{NH}_{3}+\mathrm{NO}+\mathrm{NO}_{2} \rightarrow 2 \mathrm{~N}_{2}+3 \mathrm{H}_{2} \mathrm{O}
$$


Conversely, the reaction is slower when $\mathrm{NO}_{2} / \mathrm{NO}_{\mathrm{x}}$ ratio exceeds $50 \%$ (Bosch and Janssen 1988), and such reaction, solely with $\mathrm{NO}_{2}$ is provided by the following Equation (10).

$$
8 \mathrm{NH}_{3}+6 \mathrm{NO}_{2} \rightarrow 7 \mathrm{~N}_{2}+12 \mathrm{H}_{2} \mathrm{O}
$$

In the SCR system, the ammonia decomposes on the catalyst surface and reacts with $\mathrm{NOx}$. The unreacted $\mathrm{NH}_{3}$ is stored in the catalyst through adsorption and the stored ammonia is either further reacted with $\mathrm{NOx}$ and oxidized with $\mathrm{O}_{2}$ or desorbs from the surface. The potential adsorption, desorption, oxidation and NOx conversion capability of the SCR catalyst to the ammonia depends on temperature, surface area and catalyst loading (Haga et al. 2015). For example, the amount of $\mathrm{NH}_{3}$ that can be stored in a SCR catalyst, decreases with rise in catalyst bed temperature. Additionally, the storage capacity as a function of temperature also tend to differ with the type catalyst formulation used. Eijnden et. al. showed zeolite based SCRs can store higher levels of $\mathrm{NH}_{3}$ at lower temperatures than for a Vanadium based catalyst SCR, and also indicates a non-linear behavior of storage with respect to temperature, as depicted in Figure 5 (Eijnden et al. 2009).

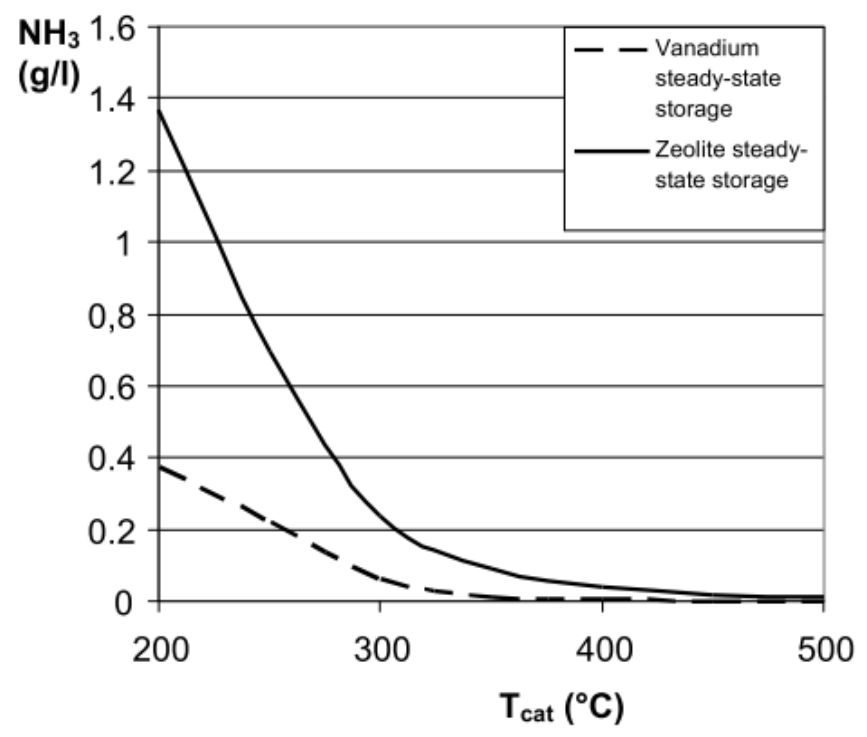

Figure 5. Ammonia storage capacity for two SCR formulation as a function of temperature (Eijnden et al. 2009) 


\section{6 $\mathrm{NH}_{3}$-SCR activity dependency}

The main target of the SCR system is the conversion efficiency of $\mathrm{NO}_{\mathrm{x}}$, and this efficiency highly depends upon multiple factors such as type of ammonia storage ratio, catalyst material, NO-to- $\mathrm{NO}_{2}$ ratio, and most importantly the catalyst temperatures at which the reaction takes place inside the SCR system (Keuper et al. 2011). Additionally, it presents that above mentioned parameters have interdependency within each other for optimum SCR functionality.

As reviewed earlier, the effect of temperature on SCR efficiency also depends on the type of catalyst coating used. Different catalysts-based material within the SCR system have varying light-off temperatures along with different temperature ranges for optimum catalyst activity. Figure 6 compares the catalytic activity of iron-based $(\mathrm{Fe})$, copper-based $(\mathrm{Cu})$ and vanadium-based $(\mathrm{V})$ coated catalysts given by the symbols $(\bullet),(\boldsymbol{\Delta})$ and $(\bullet)$, respectively.

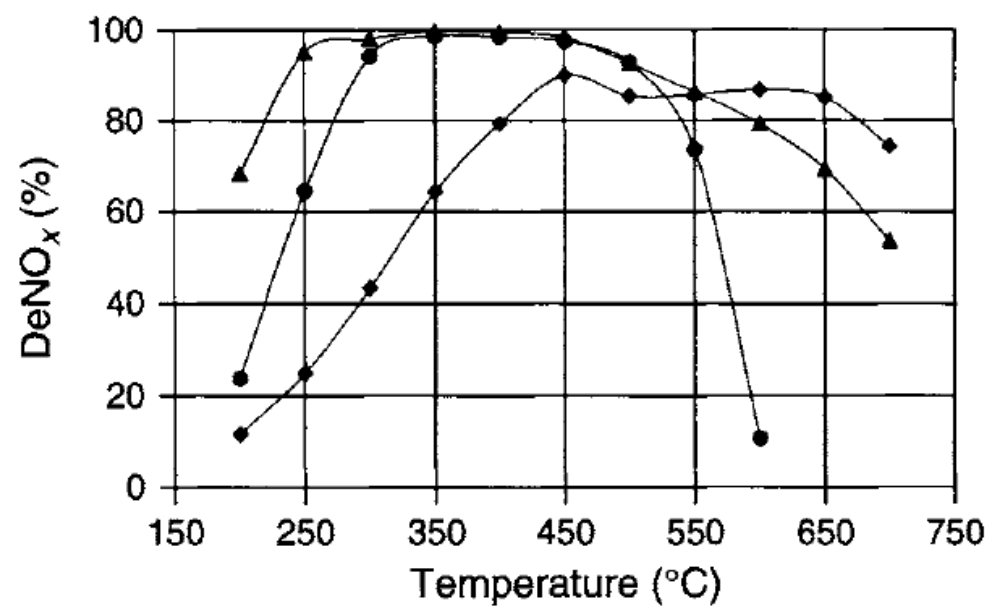

Figure 6. NOx conversion efficiency for vanadium-based and metal-exchanged zeolite-based SCR activity at varying temperatures under standard-SCR conditions

(Kröcher 2007)

A similar pathway could be approached towards a lean-burn natural gas engine application, and catalyst formulations plays a vital role in the overall performance of converting ammonia and NOx emissions. Based on different SCR formulation, the performance due to lean-burn catalytic operation can be varied with temperature, in changing the catalysts light-off temperatures (the minimum temperature at which the catalyst starts aiding the selective reaction) and the optimum conversion temperature ranges, where the catalyst effectively reacts in converting the targeted component (Colombo, Nova, and Tronconi 2010). In specific to heavy- 
duty diesel market, $\mathrm{Cu}$-zeolite and Fe-zeolite based and V-based are the two commonly used base metal SCRs that are adopted (Skaf et al. 2014). However, the Cu-zeolite based catalyst formulation are more dominantly used than compared to the other two due to its observed lower temperature performance suitable for after-treatment activity seen in modern heavy-duty diesel vehicles (Kamasamudram et al. 2010). In terms of their performances at different active temperature ranges, Cu-zeolite based have operating range between $\left(150-450^{\circ} \mathrm{C}\right), \mathrm{Fe}$-zeolite based have between $\left(350-600^{\circ} \mathrm{C}\right)$, and V-based have between $\left(300-450^{\circ} \mathrm{C}\right)$ (Majewski 2005). On the other hand, natural gas SI engines are typically known to operate at high combustion temperatures resulting in high exhaust stream temperatures. From a recent on-road study conducted by WVU with a $2013 \mathrm{CNG}$ vehicle, show exhaust temperatures as high as $600^{\circ} \mathrm{C}$ measured right after the TWC (Quiros et al. 2016). The zeolite-based SCRs seems more suitable in high exhaust temperature activity of natural gas engines; however, it is to be noted that the ammonia is a precursor of TWC reaction and its known to be generated irrespective to the aftertreatment state. Consequently, the ammonia storage capacity of the select SCR would be considered as an important factor in selecting the right formulation of a passive reduction system. A study performed by Frobert et al. indicated that the exhaust temperature, $\mathrm{NO}_{2} / \mathrm{NOx}$ ratio, and air mass flow were the three main parameters that influenced the ammonia storage characteristics (Frobert et al. 2009). Studies have also shown ammonia storage capacity tend to be higher at lower catalyst temperatures and decreases as temperature increases (Li et al. 2010). $\mathrm{Li}$ et al. specifically highlights results for Cu-zeolite based catalyst observing higher ammonia storage capacity as compared to the Fe-zeolite based catalyst, however, both catalyst showed ammonia storage at their lowest levels and an initiation of increasing trend in $\mathrm{NH}_{3}$ slippage (desorption) at temperatures of $400^{\circ} \mathrm{C}$ and above.

In contrast to any specific SCR catalyst, the $\mathrm{NH}_{3}$ adsorption and desorption characteristics highly depends upon factors like the gaseous concentration, catalyst surface temperature and the exhaust flow rates (Zhao et al. 2011; Colombo et al. 2012; I. Nova 2011; Keuper et al. 2011). Additionally, the SCR systems are highly nonlinear in behavior due to transient operations, reaction kinetics and the nature of thermal effects on the catalyst brick along with the catalysis processes occurring at different time scales (Figura et al., 2016) (McKinley and Alleyne 2009; Skaf et al. 2014). In regards to different time scales, author Skaf et al. distinguishes three time scales for an SCR system from a control point of view of $\mathrm{NH}_{3}$ dosing in their study (Skaf et al. 2014). From the three-time scale explained, the fastest time 
scale (referred to as Quasi stationary) occurs during the transport and reaction in the gas phase, which typically takes fraction of a second. The medium time scale (referred to as short term) is due to catalyst state, where effects from $\mathrm{NH}_{3}$ coverage and substrate temperature dynamics plays a role. And, then the third-time scale (referred to as long term) happens over long period of time due to sensor and actuator drifts and catalyst aging. Therefore, various control approaches have been exploited and implemented especially in performance of diesel aftertreatment devices which are oriented around the dynamics of the type catalyst system used and the performance targeted.

\subsection{Control strategies used in SCR applications}

In effective utilization of urea-SCR control technology, several advanced control techniques have been developed and studied for active urea dosage rates which are aimed at highest $\mathrm{NOx}$ conversion targets and minimum $\mathrm{NH}_{3}$ slippage. Development of such SCR controls related task require satisfying some of the key challenges of the complex after-treatment system. The sections below reviews some of the control strategies and the development in the research area of SCR control. The review also illustrates some of the control challenges, especially in today's modern heavy-duty after-treatment configuration and complexity of the systems working together to keep NOx emissions below the enforced limits.

\subsubsection{Traditional SCR control methods}

The control techniques typically involve either a feedforward open-loop control or feedback closed-loop control or combination of both architecture to improve the overall SCR performance (Haga et al. 2015; Chavannavar 2014). As described by Haga et al., the open-loop control technique which are considered as the conventional method in SCR control uses variables obtained from model based state estimation generally summarized in table lookups or maps which are defined either during offline (requiring calibration time) or even online (realtime learning/adaptive) basis. These methods are simple and less complex to implement but usually lack measurement error compensations causing inconsistency in catalyst performance over its usage time (Skaf et al. 2014). Plus, majority of the open-loop control strategies are based on the stoichiometric assumption of the SCR catalyst surface reactions which are limited due to the fact that not all the reactions taking place on the surface are known or have been identified (Chi 2009). 
On the other hand, closed-loop control technique uses feedback from its output quantity signal and, hence the control output becomes a function of the desired output error compensation, giving a dynamic control over more transient system response. Closed-loop based SCR control methods have become the prime technique in modern heavy duty aftertreatment technologies but are known to have its own challenges mainly due to its nature of dependency in measuring accurate output signals as most of the time delivered from physical sensors (Willems et al. 2007). Willems et al. mentions some of the major aspects that limits the proper applicability of the SCR performance controlling technique which are seen from slow catalyst dynamics, cross-sensitivity of the $\mathrm{NOx}$ and $\mathrm{NH}_{3}$ sensor to respective species, and time delay in the urea dosage system. The author also discusses relevant control strategies conducted via using combination of closed-loop along with open-loop control schemes as an integrated controller where the open-loop control kicks in as a fallback strategy in the case of feedback sensor failure. With similar approaches seen in SCR control systems, conventional controllers such as close-loop Proportional-Integral-Derivative (PID) controller mechanism have also widely been designed and implemented for error regime compensation along with including feedforward and adaptive compensator to overcome some of the inherent variability in the signal sources, model and hardware aging effects (Ong et al. 2010).

Furthermore, design challenges may include additional complexity underlying the dynamic changing process of the system and, eventually require rigorous tuning/calibration of the PID parameters of the control response, taking into account the control stability and the nonlinearity dependency on the temperature of the catalyst. The robustness of the closed-loop urea-SCR controllers greatly depends upon the feedback signal and its response to different transient behavior, in case of occurrence of over-dosage of urea and therefore, proper $\mathrm{NH}_{3}$ slip detection is critical.

\subsubsection{Model-based SCR control}

The characteristics of the SCR dynamics due to reaction kinetics, different catalyst state at different time scales, and strong nonlinearities originated from chemical reaction and temperature affect the adequacy of the control behavior. In a paper published by the Jiri Figura (Figura et al. 2016), the author briefly reviews different model-based estimators from numerous literature survey and identifies important set of features where the estimators have to satisfy in order to adequately characterize the SCR system and its performance. For example, the 
estimator needs to have moderate stability under feasible operating conditions, good steadystate and transient estimation, robustness to modeling uncertainties and measurement over different ambient and temperature conditions along with cost-effectiveness and low computational complexity. The author also mentions some of the proposed techniques in study and development of estimation of $\mathrm{NOx}$ concentration and $\mathrm{NH}_{3}$ coverage ratio. $\mathrm{A}$ better dynamic operation model-based control has been shown using a state estimation via $\mathrm{NH}_{3}$ coverage ratio, which is not directly measurable but can be estimated from kinetic model.

Model-based SCR control strategy based on estimating the state of the SCR system has been an effective methods in reducing design complexity, usually witnessed in classical controller methods (Herman et al. 2009). The state feedback and state estimation control feature with linear and nonlinear observer techniques have shown SCR control benefits than relying only on inlet-out SCR NOx and $\mathrm{NH}_{3}$ values, and in majority has led to sensor reduction. However, depending upon the inaccuracy of state estimation, such approach are known to sensitive to disturbance and error accumulation which causes variation in the SCR control (Skaf et al. 2014),. Simplified models on state estimation with assuming linearized SCR system, in order to predict gas phase $\mathrm{NH}_{3}$ slip and catalyst ammonia coverage ratio has also been studied (Upadhyay and Van Nieuwstadt 2006; Devarakonda, Johnson, and Parker 2012). Although such approach works well within a narrow operating conditions of the SCR, their accuracies are limited due to presence of nonlinearities, uncertainties and high variable disturbance (Skaf et al. 2014). Likewise, there have multiple studies using nonlinear models to estimate internal species along with $\mathrm{NH}_{3}$ coverage ratio. Studies have shown to use different estimation technique using non-linear models, such as sliding mode observer design for estimation of $\mathrm{NH}_{3}$ coverage ratio with incorporating temperature and inlet and out NOx sensors signals but require a mid-catalyst $\mathrm{NH}_{3}$ sensor to give robustness against the $\mathrm{NH}_{3}$ cross-sensitivity (M.-F. Hsieh and Wang 2011). Similarly, Chen et al. proposed an alternative design which did not require the $\mathrm{NH}_{3}$ sensor and estimated the mid-catalyst $\mathrm{NH}_{3}$ concentration with minimum $\mathrm{NH}_{3}$ cross sensitivity (Chen and Wang 2015). Some studies have also utilized using Extended Kalman Filter (EKF) technique as the nonlinear model estimator with different outlet sensor configuration with and without considering $\mathrm{NOx}$ sensor cross sensitivity to $\mathrm{NH}_{3}$ (M. F. Hsieh and Junmin Wang 2010; Figura et al. 2016). Numerous model-based studies have been considered with or even without using physical sensors such as the $\mathrm{NOx}$ and $\mathrm{NH}_{3}$ sensors as these sensors tend to add cost to the overall system (Chen and Wang 2014). 


\subsubsection{Control approach using Artificial Intelligence Techniques}

Currently, artificial intelligent techniques have been gaining lot of attraction in developing automotive virtual sensors mainly in the area of on-board after-treatment diagnostics, delivering similar accuracy, dynamic performance and faster time response (Arsie et al., 2013). Few examples of fuzzy-logic usage in automotive applications are: fault diagnosis for engine vacuum leak detection (Lu et al., 1998), control methods for plug-in hybrid electric vehicles (Ivanov, 2015), vehicle speed controller (Altrock, 1997), and more famously used in active vehicle suspension systems (Jinzhi et al., 2002). Some vehicle manufactures have also used it as engine-control solution in ECU architecture replacing some of the loop-up-tables (Altrock, 1997). A study published by Jinzhi et al. also demonstrated using fuzzy-logic controller with combination of other artificial technique such as GA-based optimization method for PID tuning and NN for parameter estimation (Jinzhi et al., 2002).

Similarly, fuzzy-logic decision-making strategy has also been efficiently utilized for aftertreatment controls (Niewiadomski and Kacprowicz, 2014, Meierjurgen et al., 2013, Theodore, 2008). In the field of SCR control development, Fuzzy-Logic (FL) based control algorithm has also been widely utilized in urea-dosage application (Soliman et al., 1998). In a thesis research, carried out by Theodore R. Adams from WVU (Theodore, 2008), studied a controller design developed for an open loop non-sensor based fuzzy logic urea dosage controller with employing development of urea injection maps. The study presented results with more than $50 \% \mathrm{NOx}$ reduction over two different transient cycles with maximum $\mathrm{NH}_{3}$ slippage of $10 \mathrm{ppm}$ and less. In a another study aimed at complete sensorless SCR system approach, two ANN was trained, one for predicting engine out NOx using engine parameters and the other for predicting SCR $\mathrm{NH}_{3}$ coverage ratio using a non-linear state-space plant model that simulated reduction reactions in the SCR (Meierjurgen et al., 2013). A fuzzy-logic based controller was then developed to control urea-dosing utilizing two inputs, consisting of the error function calculated from the output $\mathrm{NOx}$ of $\mathrm{ANN}$ with predetermined $\mathrm{NOx}$ target and $\mathrm{NH}_{3}$ surface coverage predicted from the second ANN.

The fuzzy-logic based control strategies can be designed for non-linear systems with just basic minimum knowledge of depended multiparametric behavior of the desired system using experimental experiences. This tends to reduce the complexity in decision-making mechanism approach by applying data-driven engineering heuristics and makes the control logic qualitative in nature. 


\subsection{Control-oriented model based on physical $\mathrm{NOx}$ and $\mathrm{NH}_{3}$ sensors}

Utilizing in conjunction with one or multiple sensors in order to monitor SCR state and activity have been common control approach, mainly in open-loop controls, in targeting efficient NOx reduction as a result of adequate urea injection upstream of the catalyst. For example, $\mathrm{NH}_{3}$ sensors has been used for pre-SCR ammonia concentration and mixing as a feedback signal to controllers for urea-dosage purpose. Similarly, such method is also further refined by including NOx signals monitoring NOx downstream of the SCR catalyst as an additional feedback signal. Combination of using NOx sensor pre-and-post of SCR also provides advantages, especially in the OBD applications. The flexibility and performance of the control related task also depends upon he integrity of the sensors used, for example, cross sensitivity of $\mathrm{NOx}$ sensor to $\mathrm{NH}_{3}$ play a key challenge in effective SCR activity. The following paragraphs reviews literature on using $\mathrm{NOx}$ and $\mathrm{NH}_{3}$ sensor for commercial SCR control applications and their ongoing development in widely available model-based and adaptive control techniques.

Similar to oxygen sensors, NOx sensors have been in development from early as 1990s, and was commercially introduced in 2002 for gasoline cars and extended onto diesel SCR system in 2005 (Jääskeläinen and Majewski 2016). The on-board applied NOx sensors have commonly been working under the same principle based on yttrium-stabilized $\mathrm{ZrO}_{2}$-based electrochemical sensing and have progressed over time on measurement ranges, accuracy and durability. Figure 7 provides the common layout of the working principle of the $\mathrm{ZrO}_{2}$-based $\mathrm{NOx}$ sensor used in common exhaust path. NOx sensor manufactured by NGK and in partnered with Continental, known as the UniNOx sensor, measures ranges of 0-500ppm or 0-1500ppm depending on the

application used for, with accuracy of $\pm 10 \%$ at $100 \mathrm{ppm}$ to $500 \mathrm{ppm}$ and $\pm 10 \mathrm{ppm}$ at $0 \mathrm{ppm}$ (NGK 2017). 


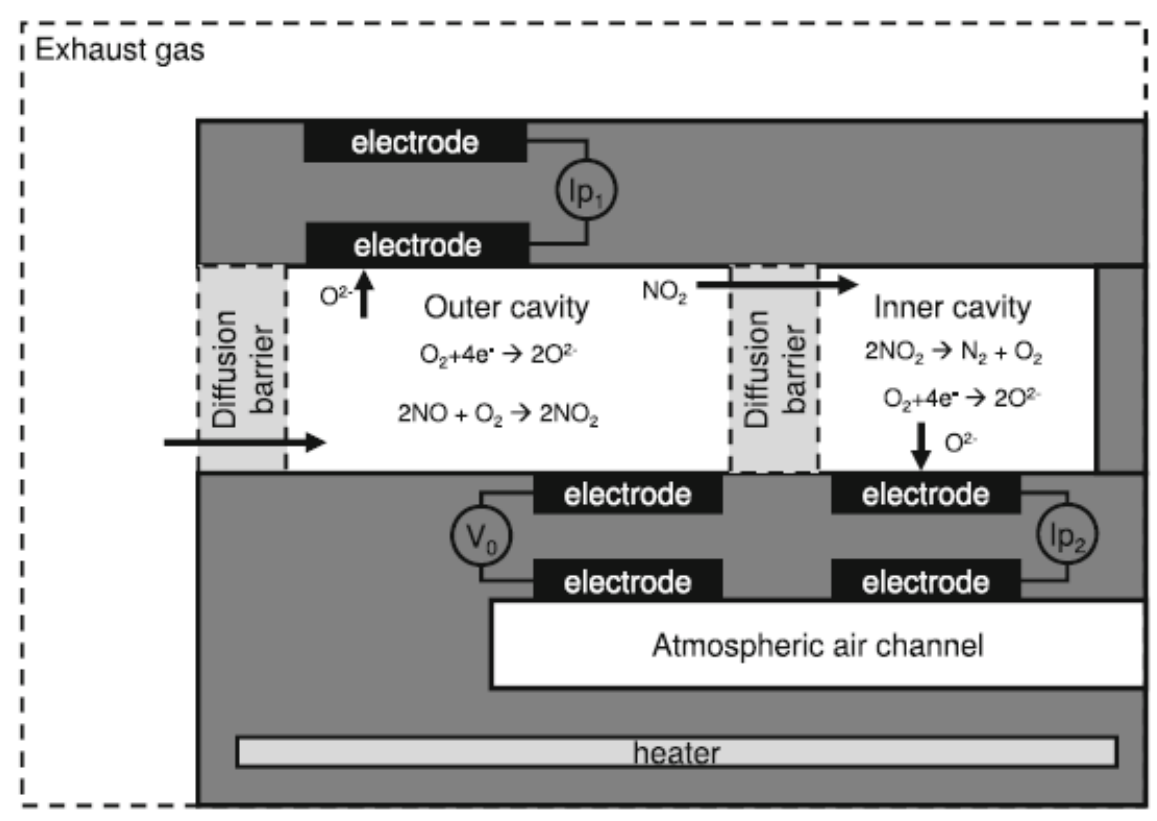

Figure 7. Schematic of a NOx- $\mathrm{ZrO}_{2}$ sensor (Blanco-Rodriguez 2014)

Current NOx sensor measurement method has a major challenge, that is such sensors are typically cross-sensitive to other nitrogen compounds at various levels. More importantly, cross-sensitivity due to gaseous $\mathrm{NH}_{3}$ have shown to corrupt $\mathrm{NOx}$ sensing measurement and varies with temperature (Zhang, Wang, and Wang 2015). This could potentially impact SCR urea control dependent upon NOx sensor feedback to accurately control at dynamic conditions of the exhaust. These could also lead to over or under dosing of urea, causing either urea cost or impact catalyst performance. There have been studies on eliminating the NOx sensor crosssensitivity to $\mathrm{NH}_{3}$ and estimate actual NOx concentration using cross-sensitivity correction factors, and typically modeled using the following expression (M. F. Hsieh and Junmin Wang 2010; Marins 2000):

$$
C_{N O, \text { sensor }}=C_{N O}^{*}+K_{C S} C_{N H 3}
$$

where, $C_{N O}^{*}$ is the actual NOx concentration, $K_{C S}$ is the cross-sensitivity factor, and $C_{N H 3}$ is the actual $\mathrm{NH}_{3}$ concentration. The model is used in predicting the actual NOx concentration using the sensor model as expressed above. The $K_{C S}$ cross-sensitivity factor is obtained using ammonia measurement. The authors address that the level of cross-sensitivity factor changes and varies between sensors. It shows that sensor aging causes inaccuracy in the 
factor, slowly with time, and becomes a challenge to develop models to adequately. Design based on Extended Kalman Filter (EKF) was proposed in the study to estimate NOx concentration and cross-sensitivity factor based on approximated model and the stochastic character of signals. Results obtained on FTP75 test cycle indicated the approach to accurately eliminate cross-sensitivity error caused by presence of ammonia and able to predict actual NOx concentration. In a recent study, the same authors utilized a different approach to remove NOx sensor ammonia cross-sensitivity from measurement in diesel SCR systems (Zhang, Wang, and Wang 2015). The study evaluates using $\mathrm{H}_{2} / \mathrm{H}_{\infty}$ approach, a design for gain-scheduling strategy consisting of an adaptive compensation for the existing nonlinearities and the involved timevarying parameters of the SCR model. Experiment results from the study indicated that the proposed model performed better over the EKF on estimating the cross-sensitivity of NOx sensor to ammonia, as shown in Figure 8. Additionally, EKF approach was also found to be slower than the alternative proposed method.

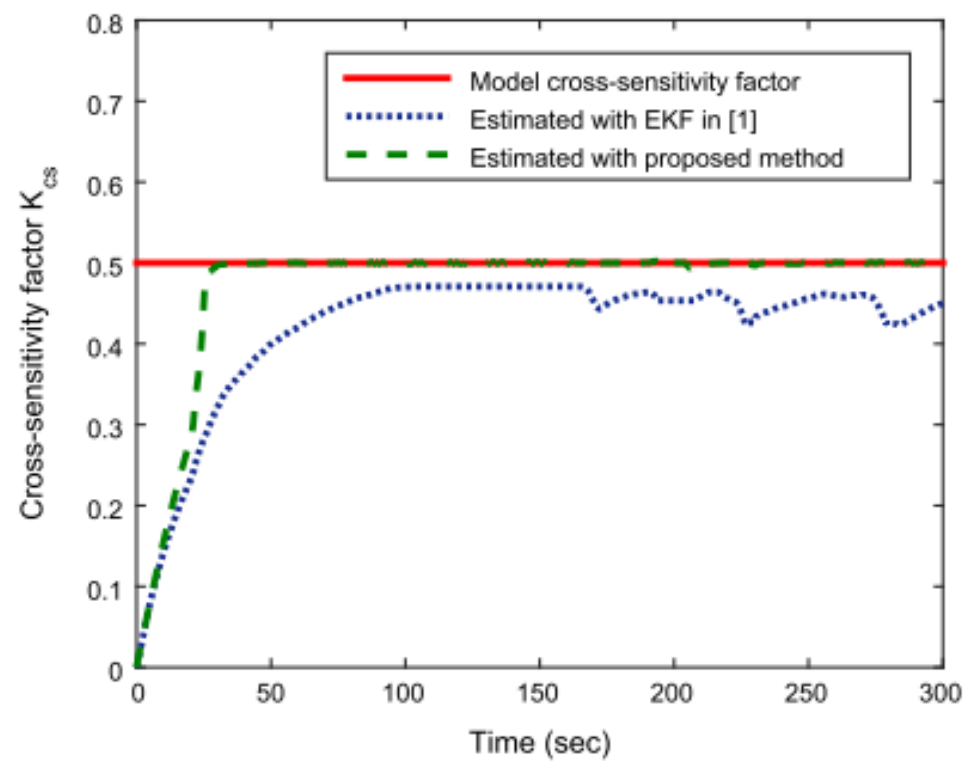

Figure 8. Performance of NOx sensor $\mathrm{NH}_{3}$ cross-sensitivity factor using different error estimation designs (Zhang, Wang, and Wang 2015)

NOx sensors have also been used to estimate $\mathrm{NH}_{3}$ concentration without requiring $\mathrm{NH}_{3}$ sensor through cross-sensitivity correlation between $\mathrm{NOx}$ and $\mathrm{NH}_{3}$. A method based on two NOx sensors with different temperature dependent $\mathrm{NH}_{3}$ cross-sensitivity characteristics at the 
SCR outlet was proposed to estimate also the SCR-out $\mathrm{NH}_{3}$ concentration (Chen and Wang 2015).
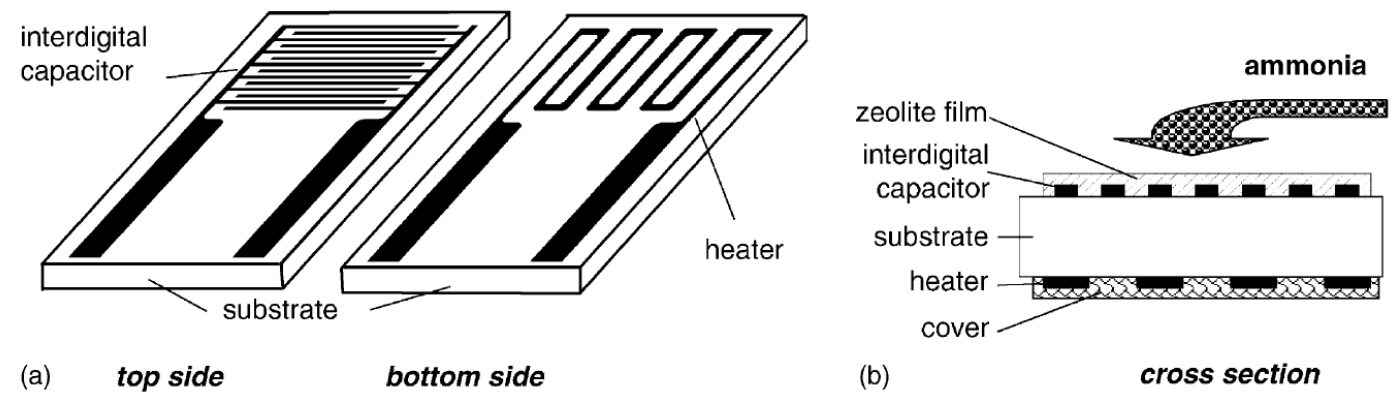

Figure 9. Schematic of $\mathrm{NH}_{3}$ sensor based on non-equilibrium electrochemical sensing principle (Moos et al. 2002)

Application of using $\mathrm{NH}_{3}$ sensors have also been widely used for measure $\mathrm{NH}_{3}$ concentration and provided benefits in developing robust SCR feedback control. The developed control strategies have also been used in conjunction with model-based approach in estimating $\mathrm{NH}_{3}$ storage capacity of the SCR as such storage characteristics is not directly measurable but including it in models benefits in state-estimation of the SCR under time and temperature dependent non-linear dynamics of the system. There have been only limited commercially developed $\mathrm{NH}_{3}$ sensors in market, operation based on a non-equilibrium electrochemical sensing principle where the sensing element used co-fired zirconia and alumina layers with $\mathrm{NH}_{3}$ sensing, a Pt reference electrode and an integrated heater circuit (Moos et al. 2002). Figure 9 presents a schematic of a commercially available $\mathrm{NH}_{3}$ sensor. Typically, the $\mathrm{NH}_{3}$ sensors are used post-SCR to detect any ammonia leak, and are designed to detect the range of ammonia in the exhaust. $\mathrm{NH}_{3}$ sensor designed by Delphi, has a measurement range of 0 to $100 \mathrm{ppm}$ with a tolerance of $\pm 5 \mathrm{ppm} \mathrm{NH}_{3}$ at $10 \mathrm{ppm}$ range (D Wang et al. 2007). However, these sensors are observed to be relatively insensitive to interference from nitrogen compounds and along with $\mathrm{HC}$ and $\mathrm{CO}$ but are reported to sensitive to interference from water and oxygen (D Wang et al. 2007). According to the study conducted by Wang et. al with the Delphi $\mathrm{NH}_{3}$ sensors, the water and oxygen tend to have inverse interference effect to the $\mathrm{NH}_{3}$ sensor. But at narrow range of water and oxygen in the exhaust, self-compensation effect is possible and is also possible with model based correction using air-fuel ratio and air humidity information. Ongoing work is being 
done for $\mathrm{NH}_{3}$ sensors for real-world applications and alternative technologies are being looked at by the industry and different research institutes.

\subsection{Summary}

When compared between diesel versus natural gas fueled vehicles in heavy-duty transportation application, diesel vehicles significantly dominates the market share (Delgado and Muncrief 2015). However, future demands in low fossil fuel dependent and low emission emitter vehicles are causing a trending technology shift, consequently resulting in popularity in the alternative fueled natural gas vehicles (Stanton, 2013). From a 2016 statistics data reported by the NGV Global, the natural gas powers roughly 24.4 million vehicles worldwide and projected to reach more than 30 million by the year 2024 (NGV Global 2017). Based on Stanton's review on development of efficient and clean engines to meet future GHG norms, an estimated $11.9 \%$ average annual growth rate is projected between 2011 and 2040 in the US. The growth in the use of heavy-duty natural gas vehicles in the US has been seen largely in centrally fueled fleets attributed by increasing domestic fuel availability and fueling stations along with increasing vehicle procurement incentives provided at different state and federal levels. Natural gas vehicles equipped with TWC system have shown to produce elevated levels of precursor ammonia emission. Gas-phase ammonia was considered to be a critical atmospheric pollutant contributing to the formation of airborne particulate matter (Renner and Wolke, 2008), threatening the ambient air quality and predominately effecting both human health (Krupa, 2003) and the ecosystem (Sun et al., 2016). The current regulation for heavy-duty engines excludes ammonia emission as a regulated criterion, however for the reasons discussed above there is critical need for ammonia reduction from heavy-duty stoichiometric natural gas vehicles.

The literature survey was conducted to identify a viable pathway in mitigating tailpipe $\mathrm{NH}_{3}$ emission produced in stoichiometric natural gas engine. Application of SCR technologies were reviewed in modern-heavy-duty diesel applications along with gasoline fueled engines to increase fuel benefits from lean-burn operation. However, exhaust temperature becomes a key challenge in maintaining overall performance of the SCR depending on the type of catalyst used. As catalyst formulations observed to be a key factor in $\mathrm{NOx}$ and $\mathrm{NH}_{3}$ performance over different temperature range, the study reviewed the three-major catalyst formulations used in SCRs. Furthermore, the study considered reviewing different traditional and AI based SCR control techniques which can be potentially utilized in developing active regeneration strategies 
to lower $\mathrm{NOx}$ and $\mathrm{NH}_{3}$. Finally, the study reviewed $\mathrm{NOx}$ and $\mathrm{NH}_{3}$ sensors from both, a physical sensor and estimator (virtual sensor) standpoint which are used for controlling the proposed passive-ARC system activity. 


\section{CHAPTER 3 Experimental Setup}

This chapter provides the general overview of the experimental setups, instrumentation and specifications used during the study. All measurement of the study presented herein were conducted at the Engine and Emissions Research Laboratory (EERL) at West Virginia University. The EERL is a part of West Virginia University's Center for Alternative Fuels, Engines and Emissions (CAFEE) and the transient engine dynamometer test cell is designed and operated according to recommendations set forth by Code of Federal Regulations (CFR), Title 40, Part 1065 (USEPA). It is to be noted that different sections of the experiments, herein, were conducted at different time due to unavailability in laboratory space and instruments required for the study. The following sections outlines details in experimental setup and procedure in order to meet the study's objectives.

\subsection{Test engine specification}

The after-treatment system analysis conducted using a stoichiometric natural gas engine on a heavy-duty engine dynamometer under controlled laboratory conditions. The study procured a commercial natural gas engine with its stocked after-treatment configuration as provided in Table 1 below.

Table 1. Test Engine and After-treatment Specification

\begin{tabular}{|r|l|}
\hline Manufacturer & Cummins \\
\hline Model $[M Y]$ & ISL-G280 [MY2008] \\
\hline Capacity & 8.9 Liter \\
\hline Rated Power & 280 hp @ $2200 \mathrm{rpm}$ \\
\hline Engine Hours & 13,800 hrs \\
\hline Fuel Type [Storage] & Natural Gas [CNG] \\
\hline After-treatment System & Three-way catalyst (TWC) \\
\hline After-treatment Strategy & Stoichiometric Combustion + Cooled EGR \\
\hline Engine Family & 8CEXH0540LBD \\
\hline NOx/PM Standards $[g / b h p-h r]$ & $0.20 / 0.01$ USEPA 2010 compliant \\
\hline
\end{tabular}

The Cummins ISL-G engines have found to be heavily used in urban fleet vehicles with major vocational application such as transit buses, refuse and food/beverage delivery trucks. The developed Cummins natural gas engine show significant advancement over other previous model year engines, in terms of fueling technology and after-treatment performance. The study 
used a 2008 model year Cummins ISL-G engine with baseline specification of 280hp rated power at $2200 \mathrm{rpm}$. The engine consisted of stoichiometrically fueling strategy with cooled EGR and was equipped with a TWC configuration which were manufacturer certified to comply to the USEPA 2010 emissions norms. The study also considered an older model high mileage vehicle to investigate the ranges $\left(\mathrm{NOx}\right.$ and $\left.\mathrm{NH}_{3}\right)$ of emission characteristics as observed from WVU's prior experience in testing natural gas vehicles. From ECU interrogation, the engine showed approximately 13,800 hours of operation before the experimental study.

\subsection{Engine laboratory setup}

The procured Cummins ISL-G engine and the stocked after-treatment system originated from a Class- 8 transit bus application vehicle, and was integrated to the engine dynamometer setup at EERL. The baseline engine experiments were conducted on a $500 \mathrm{HP}$ DC engine dynamometer capable of engine speeds up to $2500 \mathrm{rpm}$ was used for the experimental study and the baseline (engine + TWC) setup is shown by Figure 10. Due to unavailability of the 500HP dyno during the SCR evaluation, a 300HP AC engine dynamometer capable of engine speeds up to 3000 RPM was used for the remainder of the study. The baseline setup with the added SCR system is shown in Figure 11.

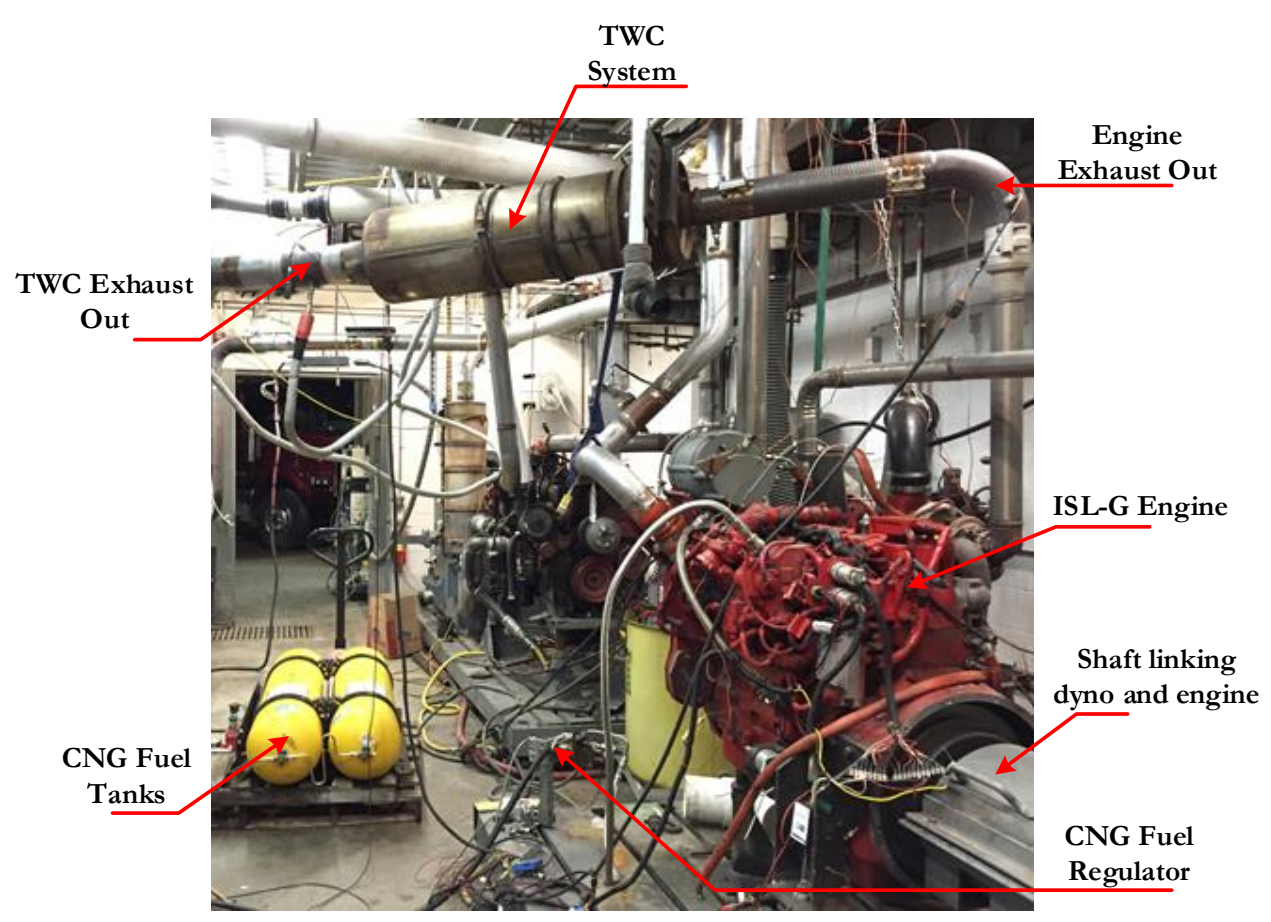

Figure 10. Baseline Cummins ISL-G test cell setup 
The test engine was coupled with the test cell dynamometer via a universal joint dynamometer shaft adapted to the engine flywheel. Throttle input and speed control were provided using WVU CAFEE's in-house engine dynamometer test cell software. CAN bus communication with SAE J1939 protocol was used between the test cell controller and the engine control unit ECU. The study conducted engine mapping procedure in order the map the engine to its rated power and torque, and verify proper functionality of the engine. Constant monitoring of the engine and after-treatment fault codes were also made to insure no fault detection detected during operating of the integrated systems.

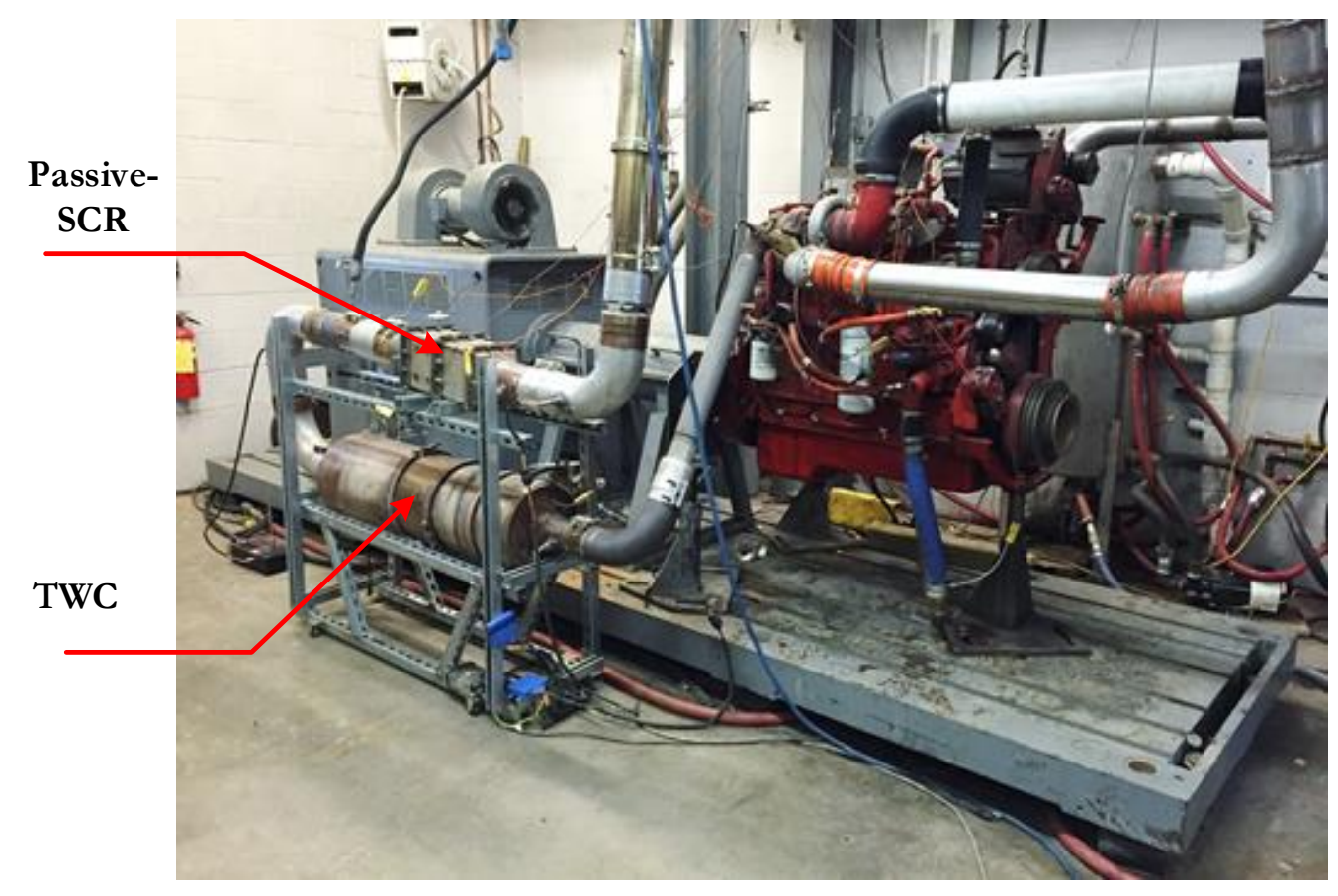

Figure 11. Cummins ISL-G (TWC+SCR) test cell setup

\subsection{Gaseous emission measurement system using FTIR analyzer}

There are numerous methods and systems commercially available in the current market to analyze various levels of constituents of gases in the exhaust from combustion sources. In order to characterize the exhaust species as raw measurement, the study utilized the MultiGas ${ }^{\mathrm{TM}}$ 2030 Fourier-Transform Infrared Spectroscopy (FTIR) continuous gas analyzer. The FTIR works in the principle of adsorption spectroscope and measures high spectral resolution data over a wide spectral gas range which had the gas speciation capability of 20 different exhaust 
species at a time. The FTIR consist of two infrared beams which originates from the same infrared source but sent to two different optical paths that varies in beam frequency, where one beam gets reflected onto a fixed mirror and the second onto a vibrating mirror as shown in the Figure 12. The combined beams are then passed through the gas chamber or cell where the gas molecules absorb energy at different levels based on spectral distribution of the molecules. Finally, the beam exiting the chamber is sent back to the detector to yield an interferogram. Fourier-transformation, a mathematical method is then used to obtain the intensity distribution of the interferometer as a function of frequency and wavelength. Intensity distribution in the infrared adsorption spectra of different gases are calibrated and given by the manufacturers and concentration of each gas measured during the continuous sampling are obtained using the preloaded calibrations.

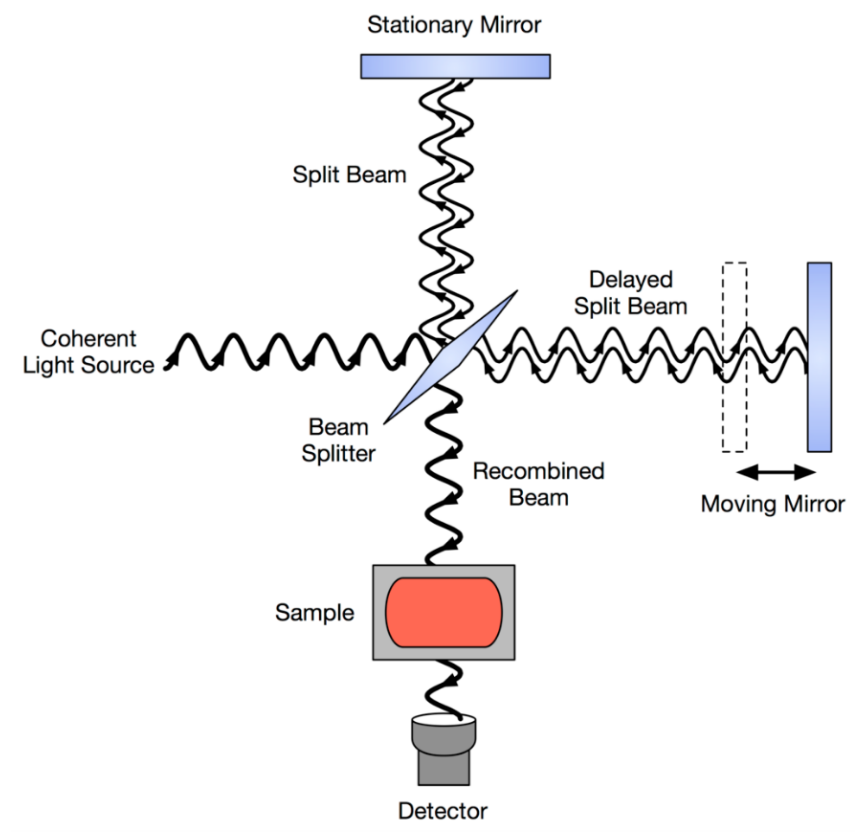

Figure 12. Schematic diagram of an FTIR interferometer (Sanchonx 2017)

The FTIR used in sampling the exhaust gases measured concentrations at 5 measurements per second $(5 \mathrm{~Hz})$ at parts per million (ppm) level. A pre-calibrated natural gas method obtained from the manufacture was used which typically consist of traditional emission components with ranges observed in natural gas engine exhaust. Since, the major objective of this study was to characterize $\mathrm{NH}_{3}$ and $\mathrm{NOx}$ before and after the after-treatment systems, the FTIR additionally had the capability of also measuring complete speciation of oxides of nitrogen 
compounds, such as $\mathrm{NO}$ and $\mathrm{NO}_{2}$. The analyzer did not measure monoatomic molecules such as $\mathrm{O}_{2}$ and $\mathrm{N}_{2}$, that's also present in the exhaust.

In order for the FTIR to continuously sample and measure emissions under transient and steady-state engine operating conditions, a sampling setup was built to draw part of the exhaust at a flow rate of (10-12) liters per minute and maintain the FTIR's cell pressure close to $1 \mathrm{~atm}$ (as per manufacturers specification). Figure 13 provides the FTIR instrument with the inhouse built sampling setup. The setup consists of a dual head diaphragm pump, which was specifically used to minimize sample pulsation and provide low flow characteristics required by the analyzer. The entire sampling lines carrying the exhaust gases entering and exiting the FTIR were controlled and maintained at $191^{\circ} \mathrm{C}$ temperature specification to avoid sample condensation. Additionally, the sampling setup also included a heated filter which was maintained at $191^{\circ} \mathrm{C}$ temperature. The heated filter was used to capture any solid particles entering the FTIR gas chamber to avoid beam obstructions. More detailed information of the FTIR specification used in the study are provided in APPENDIX A.

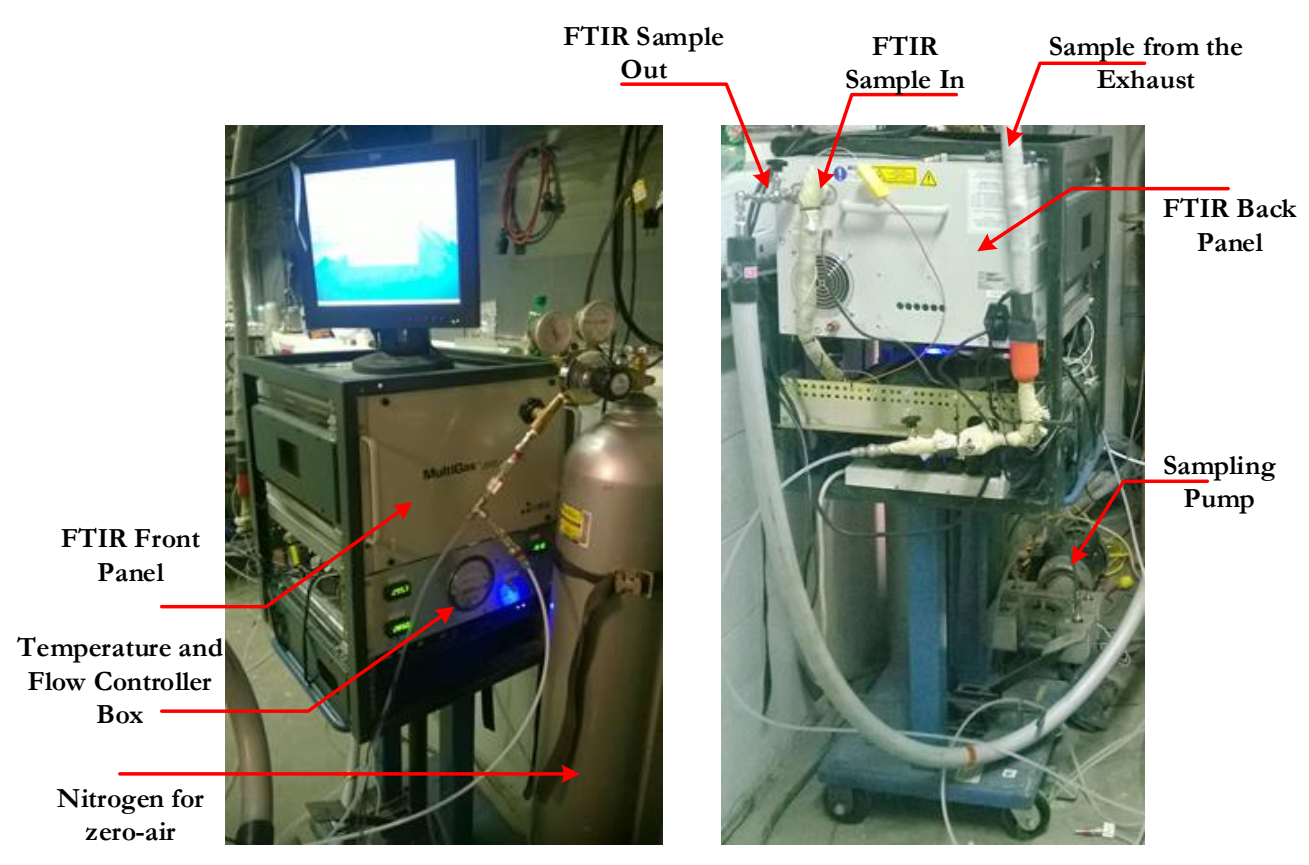

Figure 13. FTIR sampling setup

\subsection{Gaseous measurement system using CVS method}

The study also utilized the EERL's gaseous emission measurement system designed to be capable of measuring continuous engine exhaust under Constant Volume Sampling (CVS) 
method. Under this method the gaseous exhaust emission were measured on diluted basis using a full-flow constant volume sampling dilution tunnel as recommended by the Code of Federal Regulations, Title 40, Part 1065 (CFR40/1065/SubB 2017). The schematic of the gaseous sampling setup using CVS system is shown in Figure 14. The diluted samples are extracted from the dilution tunnel and routed via $191^{\circ} \mathrm{C}$ maintained heated line into the gaseous emission gaseous analyzer. Regulated gaseous emissions, including $\mathrm{CO}$, THC, $\mathrm{NOx}$ as well as $\mathrm{CO}_{2}$, $\mathrm{NO}$ and $\mathrm{CH}_{4}$ were measured using a Horiba ${ }^{\circledR}$ MEXA 7200D automotive emissions analyzer system which is also shown in the schematic below. The analyzer uses methods such as non-dispersive infrared (NDIR) to detect $\mathrm{CO}$ and $\mathrm{CO}_{2}$, chemiluminescence for $\mathrm{NOx}$ and $\mathrm{NO}$, and heatedflame ionization detector (HFID) for THC and $\mathrm{CH}_{4}$. The analyzer system did not have the capability of detecting $\mathrm{NH}_{3}$ emission. $\mathrm{CO}, \mathrm{CO}_{2}, \mathrm{NO}$ and $\mathrm{NOx}$ are measured using dry method (removes water in the sample using chilled mirror maintained below sample dewpoint), whereas THC and $\mathrm{CH}_{4}$ are measured wet. All analyzers were verified for operation as per Code of Federal Regulations, Title 40, Part 1065, Subpart D (CFR40/1065/SubD 2017) including manufacture's specifications.

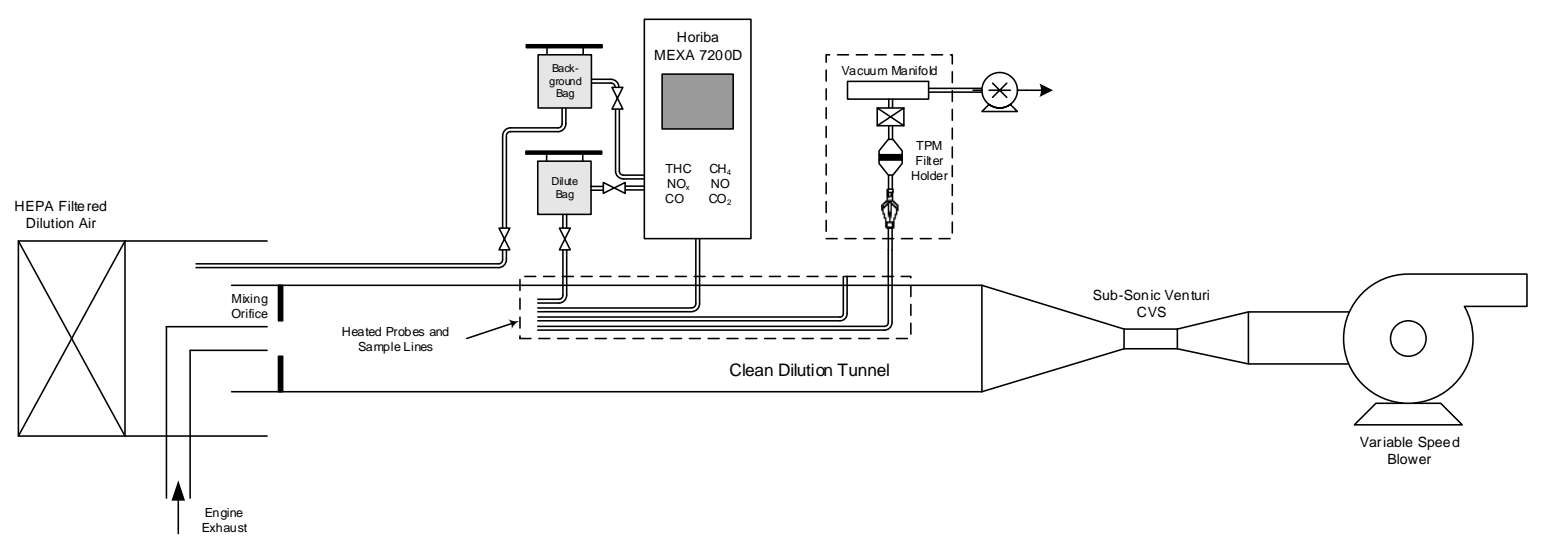

Figure 14. Schematic of dilute gaseous measurement system using CVS method

\subsection{Engine lean-ON operation control setup}

The tested stoichiometric engine used an oxygen sensor feedback signal to control the engine AFR. Since there were no access over the engine's ECU parameters, the study used an external signal that was designed to provide necessary feedback signal to the ECU in order to operate the engine into a modified AFR ratio when compared to baseline operation. This was performed by intercepting the original $\mathrm{O}_{2}$ sensor signal using an external hardware-in-loop (H- 
I-L) setup built for the study. The H-I-L setup basically consisted of using an Arduino DUI board with a relay shield and a National Instrument's (NI) multifunction I/O USB DAQ device (provide in Figure 15). The breadboard was used to connect pins between the Arduino, NI DAQ and the engine's $\mathrm{O}_{2}$ sensor signal wires.

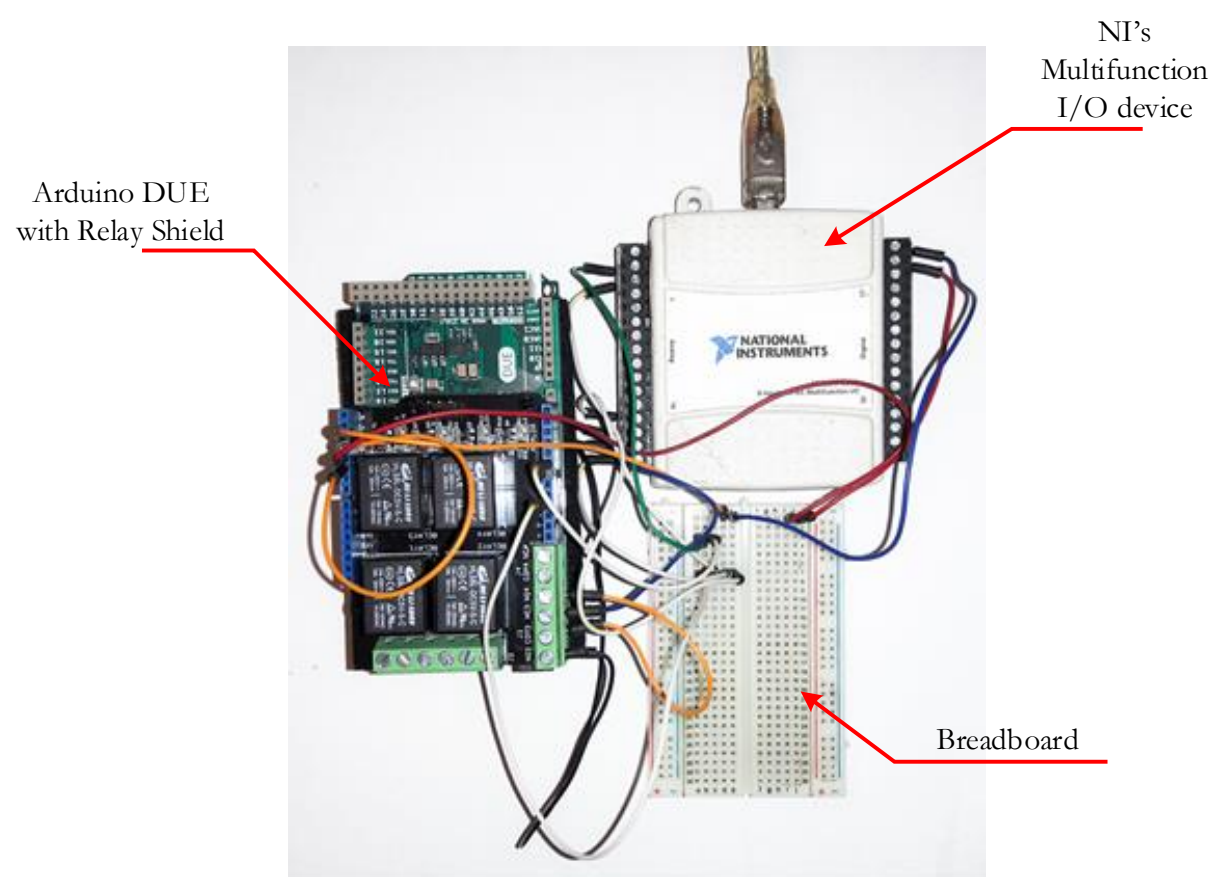

Figure 15. Manual lean-ON hardware control setup

Figure 16 presents the schematic showing the technique used in manually switch between the modified (shown by the blue signal) and the on-board $\mathrm{O}_{2}$ sensor signal (shown by the red signal). The Arduino board was used to code the voltage profile signal curves and a relay switch (added Arduino's Relay Shield) on the Arduino board was incorporated to switch between the baseline signal and the modified signal voltages. The power to switch the relay control was provided by NI's USB-6009 DAQ device and manually trigger using NI's device monitor, also shown in the schematic (referred to Figure 16). The stoichiometric engine utilized a narrow-band $\mathrm{O}_{2}$ sensor as a feedback signal which typically generates up to about 0.9 volts for fuel rich mixture and about 0.1 volts for fuel lean mixture based on the exhaust oxygen content. Although, different manufactures use different air fuel ratio strategy to dither the exhaust $\mathrm{O}_{2}$ sensor signal between the two voltages with certain amplitude and frequency, the study used dithering method with constant voltage of $0.8 \mathrm{~V}$ to approximate rich AFR condition and $0.2 \mathrm{~V}$ for lean AFR condition, which were selected based on ECU reading using Cummins INSITE 
engine diagnostic tool. The desired engine lean AFR operation was obtained based on the profile of the modified signal. Providing a dithering profile with higher duration of $0.8 \mathrm{~V}$ and a lower duration of $0.2 \mathrm{~V}$ converted the engine's ECU to interpret the engine's operation to be at rich side, hence eventually shifting the AFR to a leaner operation. Time of $2000 \mathrm{~ms}$ for rich-duration and $500 \mathrm{~ms}$ for lean-duration were found appropriate for the lean-ON operation trigger which was also based on observing the engine stability. High engine power fluctuation due to due fueling destabilization was the main visually inspected criteria used in maintaining the engine stability during the lean-ON operations.
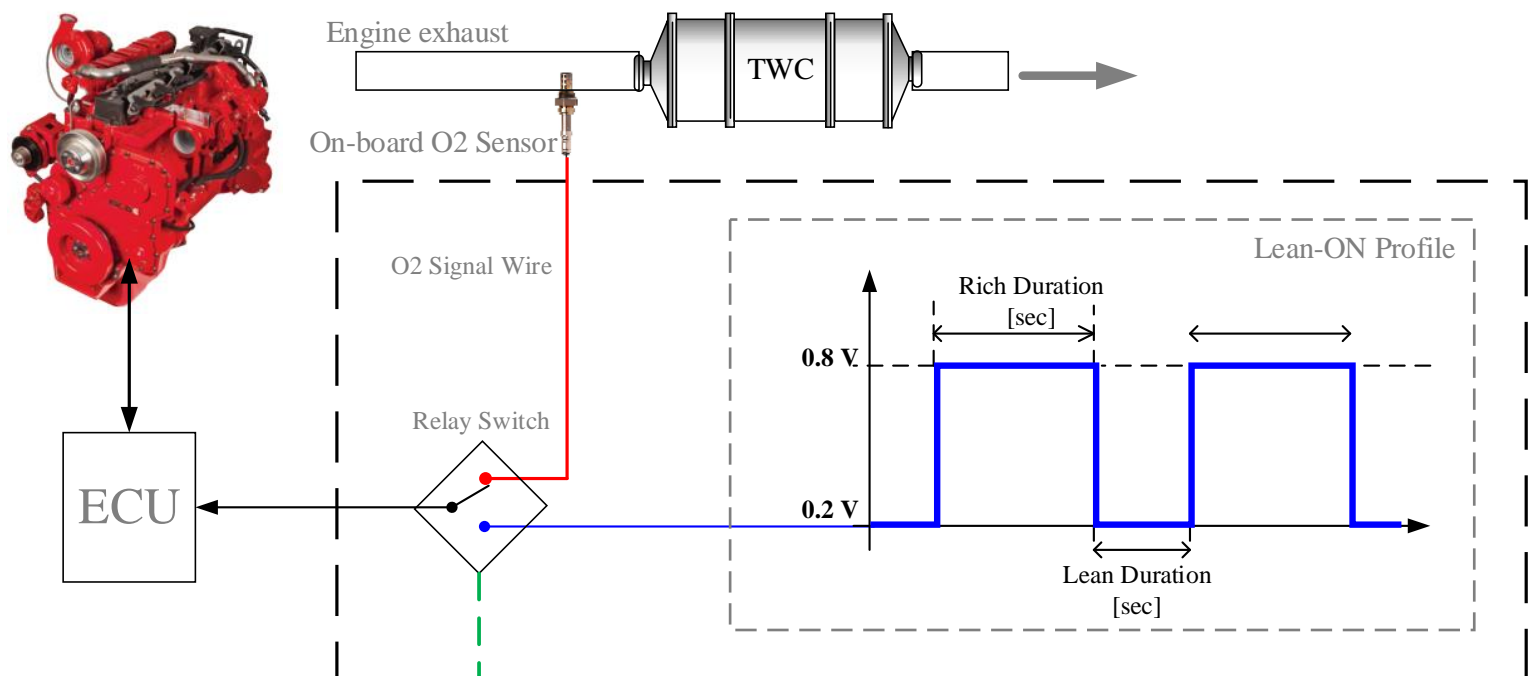

Arduino DUE Module
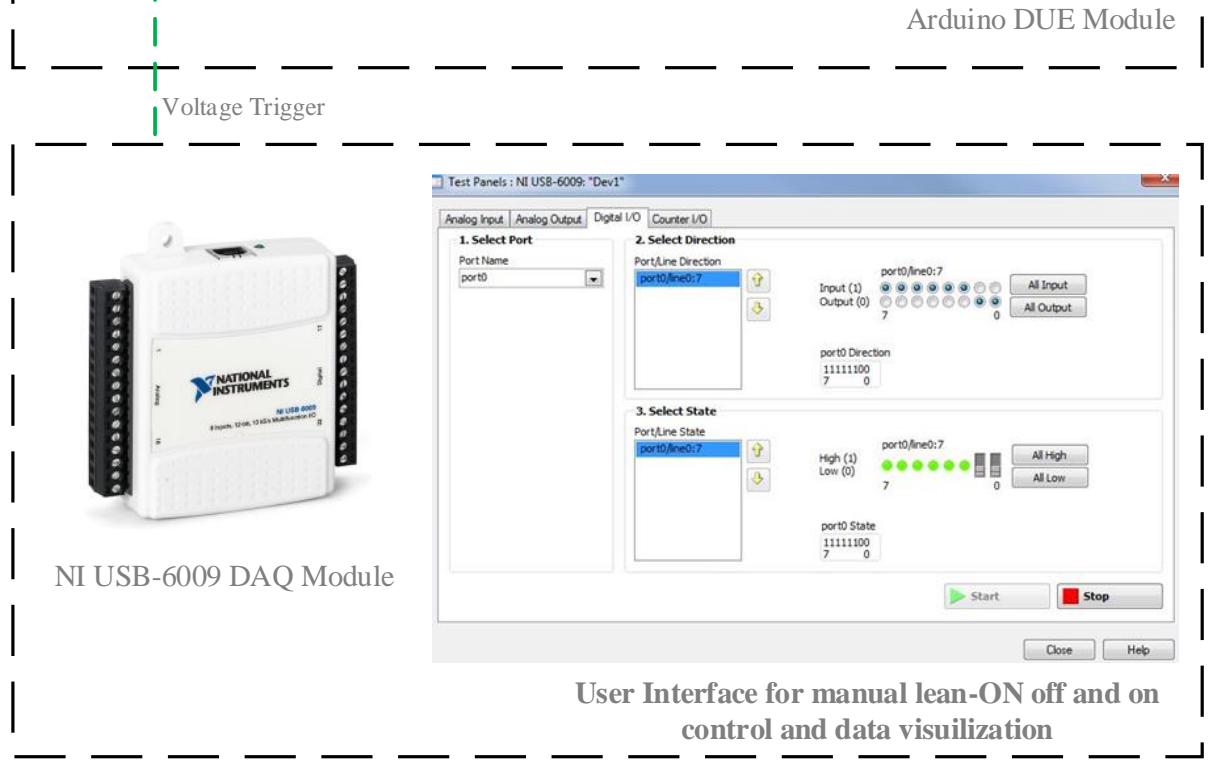

Figure 16. Schematic of manual lean-ON control setup for intercepting on-board $\mathrm{O}_{2}$ sensor signal to the ECU 


\section{CHAPTER 4 Approach}

The study evaluated a stoichiometric natural gas engine equipped with a TWC system for emission performance, and investigated feasible pathways required to control precursor ammonia emission observed from such vehicles. This chapter presents and discusses the studies approach in evaluating a 2008 Cummins ISL-G engine equipped with a stock TWC system and analysis required to investigate a SCR system to capture $\mathrm{NH}_{3}$ emission downstream of the TWC. To minimize slippage of $\mathrm{NH}_{3}$ from the proposed passive-ARC catalyst, the study extends its work with an engine based control strategy. The chapter further details the studies approach in estimating $\mathrm{NH}_{3}$ emissions after the TWC during different engine operating conditions and $\mathrm{NOx} / \mathrm{NH}_{3}$ and $\mathrm{NH}_{3}$ storage capacity from the stand-point of control-oriented and sensorless based application.

\subsection{Baseline after-treatment evaluation}

The baseline engine performance with the TWC system was evaluated under engine's dynamic operation to better understand the secondary $\mathrm{NH}_{3}$ production characteristics. Figure 17 depicts the schematic of the baseline experiment setup required for conducting the baseline engine plus TWC combined system. Inlet and outlet exhaust gas path of the TWC was instrumented with k-type thermocouples for temperature measurement. Two FTIR analyzer were setup to sample and measure up to 20 different exhaust constituents before and after the TWC system. The exhaust outlet of the TWC was routed to the CVS dilution tunnel to evaluate total emissions based on iterative carbon balance method for the baseline engine and aftertreatment configurations.

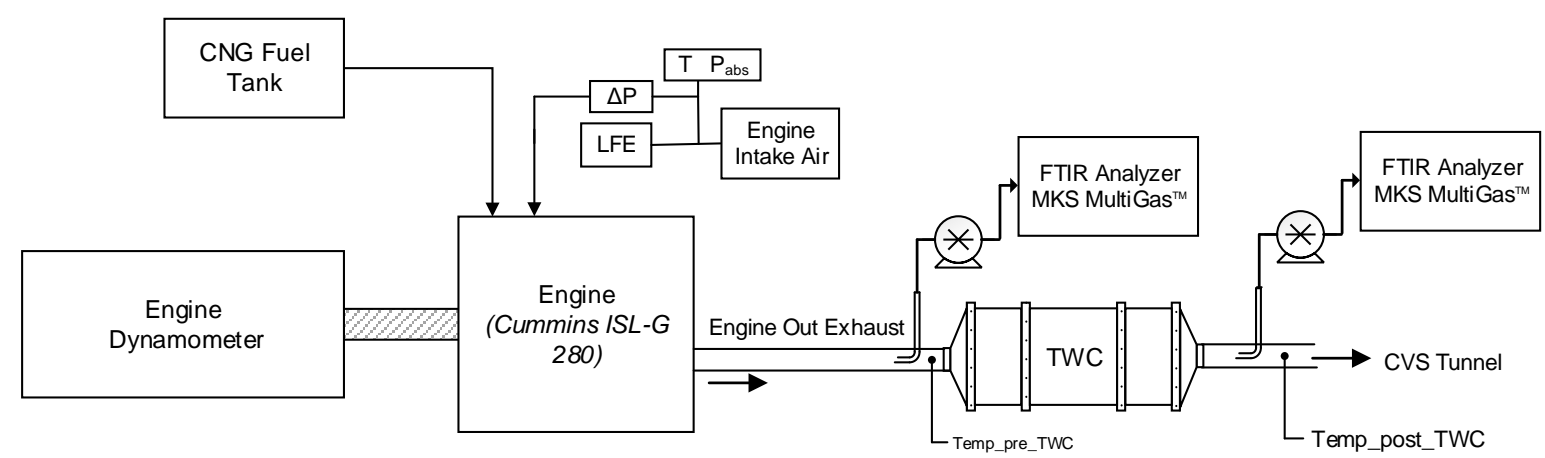

Figure 17. Schematic of the experimental setup for the baseline (engine + TWC) evaluation 


\subsubsection{Transient test cycles}

For the dynamic evaluation of the baseline setup, two heavy-duty transient cycles, Federal Transient Procedure (FTP) cycle and the World Harmonic Transient Cycle (WHTC) were selected. The cycles consisted of 1208 seconds and 1800 seconds of engine operation for the FTP and WHTC cycles, respectively. The normalized traces of the speed and torque profiles are given by Figure 18 for the FTP cycle and by Figure 19 for the WHTC cycle.

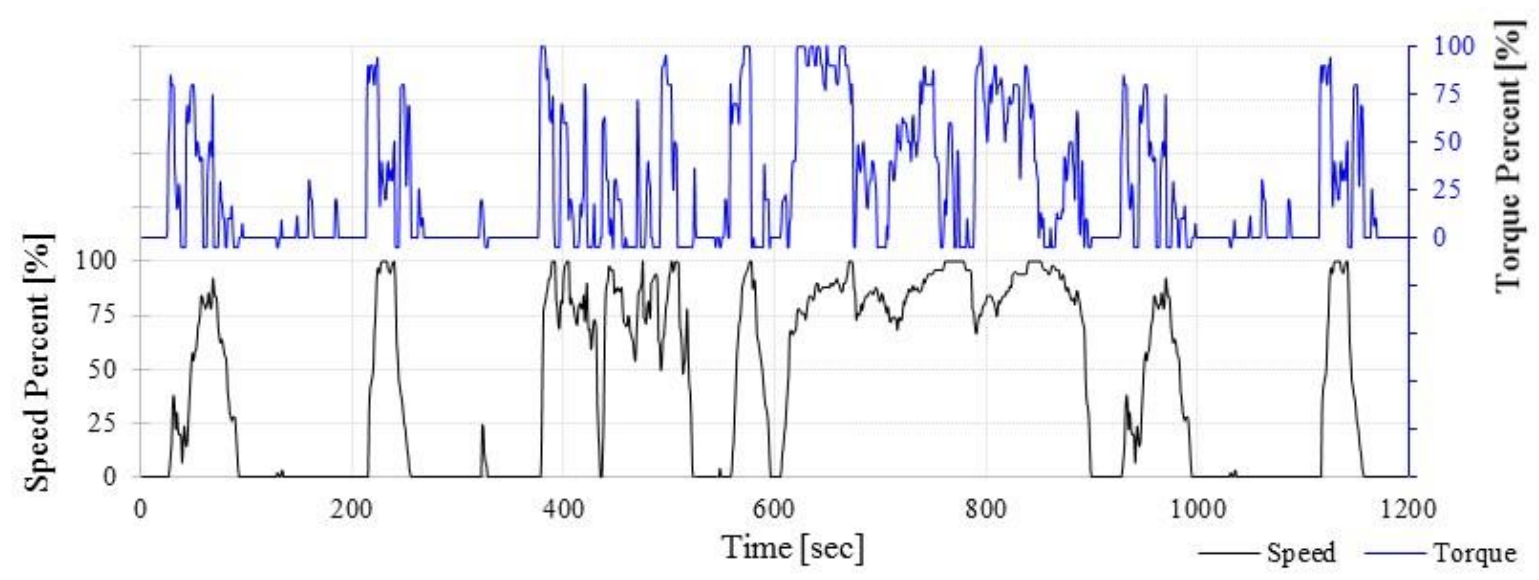

Figure 18. FTP normalized speed and torque profiles

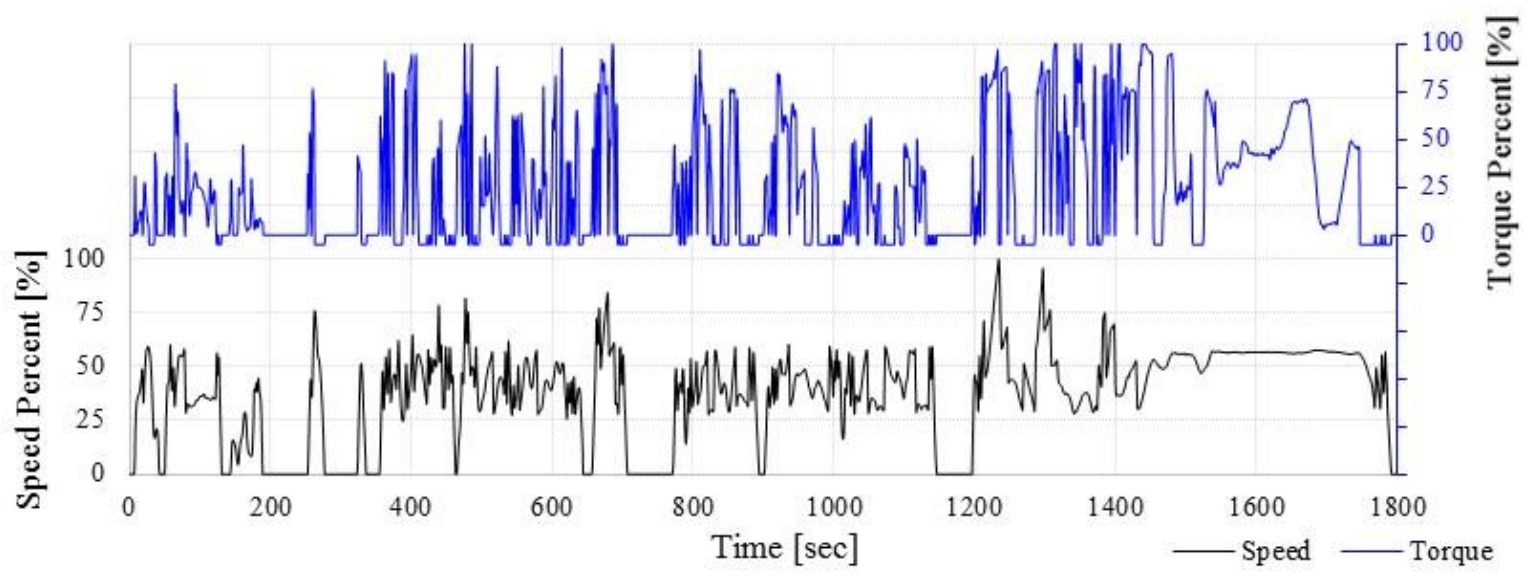

Figure 19. WHTC normalized speed and torque profiles

In the US, the FTP cycle is commonly used for regulatory emission testing of heavyduty engines, where the cycle was specifically designed to represents variety of heavy-duty truck and transit bus driving conditions, typically seen in urban city roads and highways. On the other hand, the WHTC cycles are used internationally as an engine dynamometer testing procedure, and the cycle was designed to represents world-wild pattern of real heavy commercial vehicles. 
The study selects these two cycles based on the two distinct characteristics observed between the engine speed and load combinations. In specific to engine operating conditions, majority of the FTP's points operated at a higher speed of (70-100\%) band width whereas for the WHTC lie between engine speeds of (30-60\%) band width, as depicted from the normalized points in Figure 20. The two cycles would potentially result in different output results both in fueling and emission characteristics. Additionally, also providing different range of temperature profiles which would assist further in evaluating after-treatment performance. Actual engine speed in rpm and engine torque in ft-lbs are calculated from the normalized FTP and WHTC profile using maximum torque curves obtained during the engine mapping procedure. The motoring torques were also corrected to simulate the actual engine friction torque obtained from the engine-dynamometer motoring test procedure.

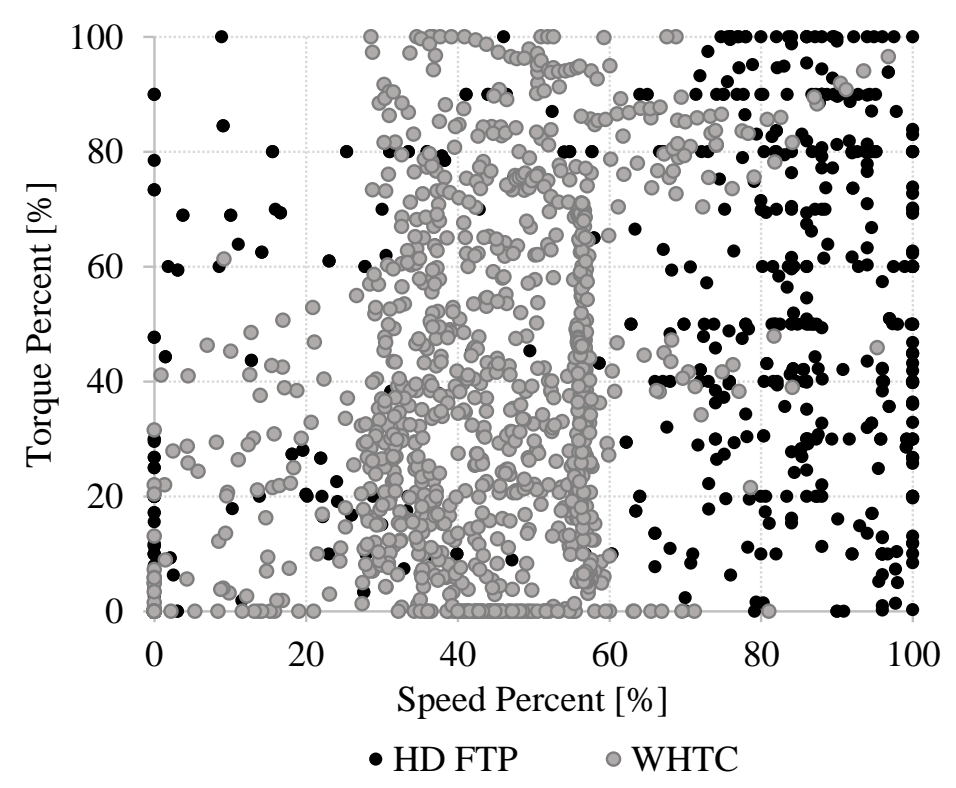

Figure 20. Normalized distribution of engines operating points for the FTP and WHTC cycles

\subsubsection{Steady-state test matrix}

For the steady-state evaluation of the engine and the after-treatment performance, the study selected twenty mode engine operating conditions, where each mode was given four minutes of stabilization time to reach a steady after-treatment state. A 20-mode test matrix was developed from using methodology based on Design of Experiment (DOE) with a space-filling design approach to obtained efficiently spaced operating points under the engines maximum 
torque curve (engines overall operating range). The space-filling design was generated and analyzed using a statistical software called $\mathrm{JMP}^{\circledR}$, where the approach efficiently characterizes operating points for a wide area of operating modes within the engines given boundaries. Engine speed and torque was used as two boundary parameters for the space-filling DOE design factor which were segmented down into multiple levels after the normalization process. The design efficiently selects a specified number of combination points (20-Mode) considering maximizing the minimum distance between points within the space of the two-normalized speed and torque boundaries. Figure 21 shows the 20-Mode points (labeled in circles) selected by the space-filling DOE design under the maximum torque curve (shown by black curve in the figure). Along with points within the engine's operating region (area in grey), the test matrix also added four more operating points (Mode 12,16,19 and 20) on the maximum torque curve.

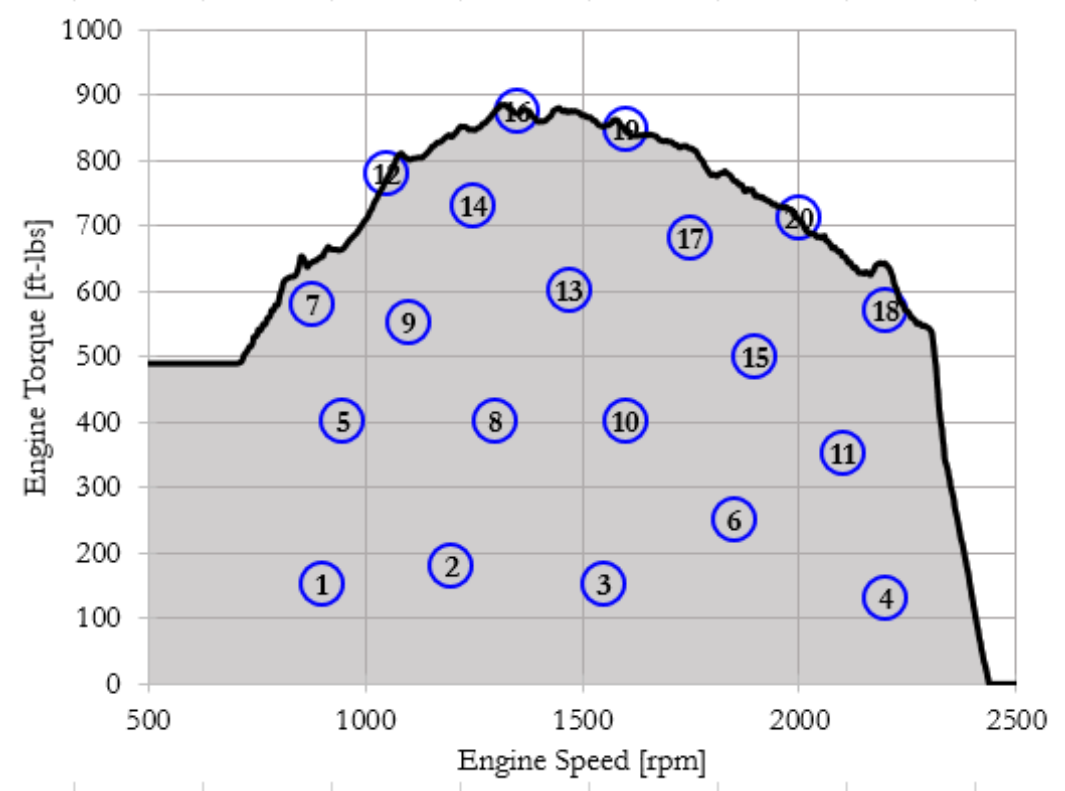

Figure 21. 20 engine operating modes for steady-state evaluation

\subsection{TWC+SCR after-treatment system evaluation}

Different SCR systems downstream of the TWC exhaust were installed for the ammonia reduction catalyst evaluation. A similar setup as described in Section 4.1 was used for the SCR evaluation but the two FTIR's were used to sample exhaust gases before and after the added SCR system. Figure 22 depicts the schematic of the test setup for passive-SCR system. The 
exhaust outlet of the SCR was routed to the CVS dilution tunnel for evaluating total emissions of the combined engine and after-treatment configurations.

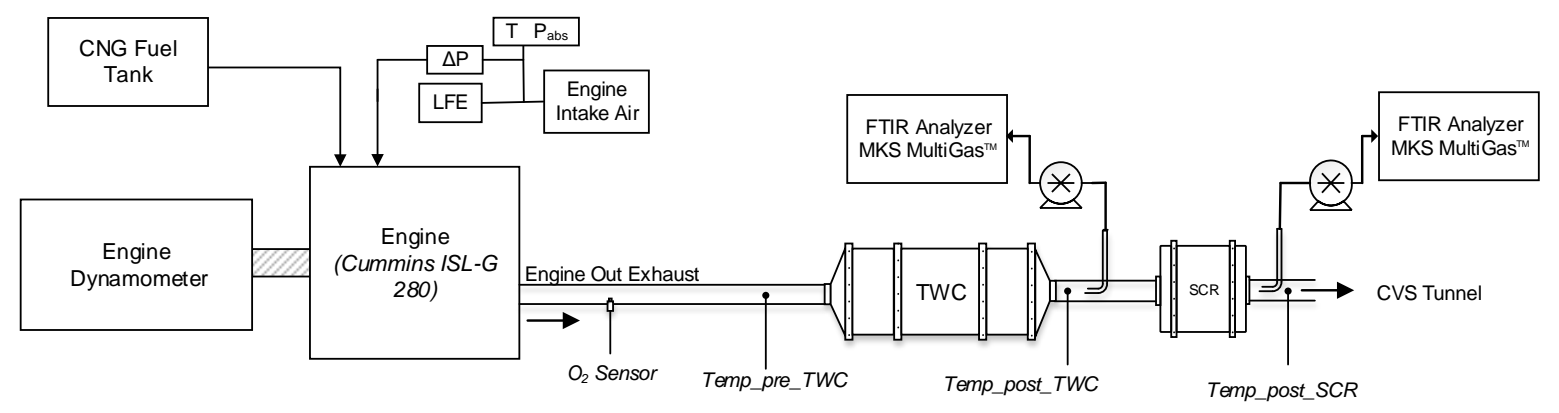

Figure 22. Schematic of the experimental setup for the (engine + TWC + SCR) evaluation

\subsubsection{Types of SCR formulation}

The study conducted five SCRs with three types of catalyst formulations that are commercially used and well-studied in the field of heavy-duty diesel applications. Four canned SCR catalysts were directly procured from two different catalyst manufacturers, i.e. Corning Inc. and AP Exhaust. Only two type of SCR catalysts (Fe-based and V-based) were available from the two manufactures. Since it was important for the study to also evaluate a widely used $\mathrm{Cu}$ zeolite based SCR catalyst by the heavy-duty OEMs, a stocked SCR system manufactured by Cummins Inc. was also included in the list of tested SCRs. Based on the manufactures specification, the Cu-zeolite based SCR catalyst which was directly procured from a heavy-duty diesel vehicle consisted of a bigger volume catalyst. The specifications of the SCR catalysts tested are provided in Table 2.

Table 2. List of five experimented SCR catalyst for ammonia reduction evaluation

\begin{tabular}{|c|c|c|c|c|}
\hline SCR Number & Manufacturer & Catalyst Type & Cell Density [cpsi] & Volume [Lt] \\
\hline SCR 1 & AP Exhaust & Fe- Zeolite & 100 & 7.07 \\
\hline SCR 2 & AP Exhaust & Fe- Zeolite & 400 & 6.39 \\
\hline SCR 3 & Corning & Fe- Zeolite & 400 & 7.83 \\
\hline SCR 4 & Cummins & Cu- Zeolite & 400 & 17.04 \\
\hline SCR 5 & AP Exhaust & V-based & 400 & 6.39 \\
\hline
\end{tabular}




\subsubsection{SCR evaluation test matrix}

The study then selected one out of the five tested SCR formulation for the development of the regeneration control logic and mode-based prediction, which are discussed in the following sections. A specific SCR was selected based on the performance in $\mathrm{NH}_{3}$ and $\mathrm{NOx}$ characteristics at different temperature ranges over the transient FTP cycle. $\mathrm{NH}_{3}$ storage adsorption and desorption characteristics were evaluated within binned temperature ranges, averaged over the three repeated FTP cycles. NOx conversion efficiencies were also evaluated over the defined temperature bins.

The study further investigated the selected SCR as the passive-ARC candidate over different steady-state operation with less number of modes as opposed to the 20-mode steadystate experiments as detailed in the TWC evaluation. The steady-state modes were selected representing the actual engine operation of the real world on-road vehicle activity. An on-road data-set collected from a similar natural gas engine equipped in transit bus application conducted for PEMS evaluation under a WVU CAFEE research program, was used to obtain specific steady-state modes. Figure 23 depicts the engine speed and torque distribution concatenated from multiple selected on-road tests which include low-to-medium vehicle speed urban-driving to high-speed highway driving activity. Eight steady-state modes representing different engine operation were picked from the engine speed-and-torque distribution based on a data clustering method where the clustering algorithms adequately groups engine speed/toque combination points to a desired number of groups. The k-mean clustering method was used for the grouping of the points where the algorithm utilizes a heuristic data classification approach to gather the data set into ' $k$ ' number of clusters based on the measure of single mean vector method. The data set are assigned into the nearest mean (centroid of clusters) using the Euclidian distance as a metric, where the sum of distances with respect to each data point in a cluster is minimized. The result from clustering the on-road engine speed and torque distribution are shown in Figure 23, where the points $(\mathrm{x})$ represent the centroid of each cluster (colored spheres), and a total of eight clusters were selected for the study. The size and shape of the colored eclipses represent the data density with $95 \%$ data coverage. The eight clustered central data points were considered as the representative steady-state modes to be conducted using the same SCR evaluation setup as discussed in the previous sections. Two-minute stabilization time was given for each mode to have an adequate stable reading of both after-treatment temperatures and overall emission constituents. 


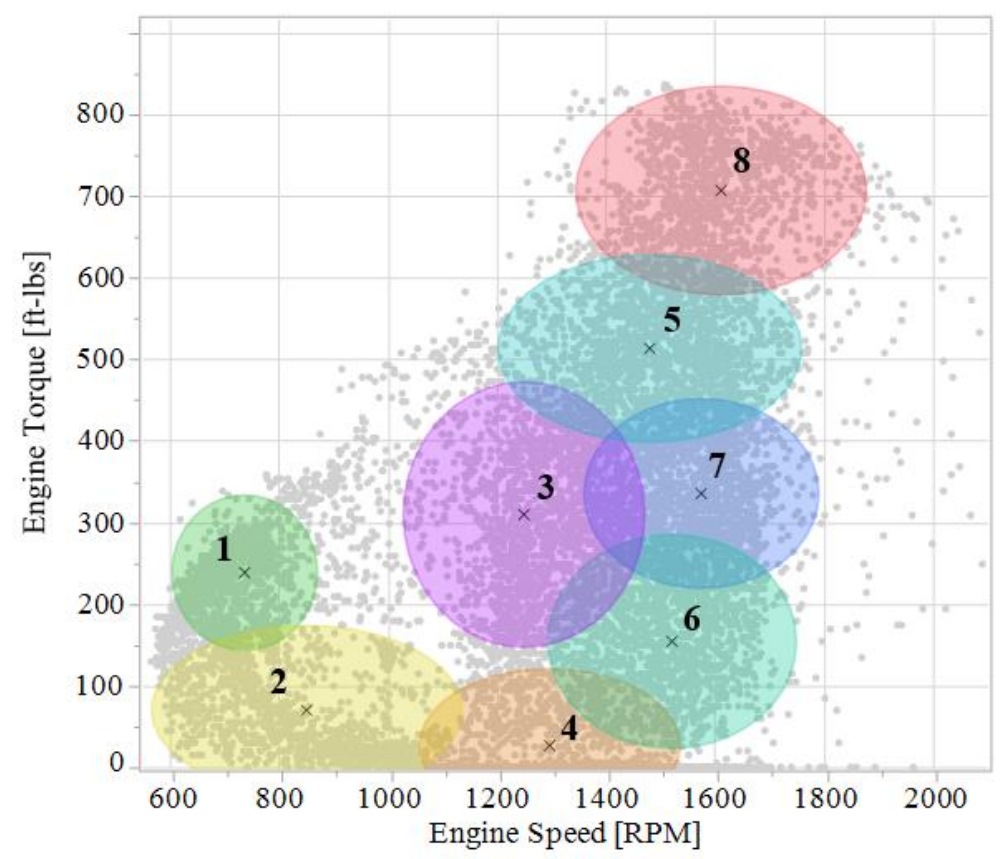

Figure 23. Transient bus real-world engine operation and 8-mode steady-state from clustering method

\subsection{Fuzzy-logic based control method}

This section provides the design overview of the engine-based control approach that works on minimizing overall $\mathrm{NOx}$ and $\mathrm{NH}_{3}$ slippage after the passive-ARC system. With the complexity of the approached configuration, a decision-making algorithm based on AI's fuzzylogic controller was designed to trigger regeneration command for lean-operation in order to meet two basic goals; (1) reduce ammonia slip from the passive-ARC system with induced NOx regeneration (lean-ON modes) intervals, and (2) maintain low to zero NOx emissions over the entire duration of operation. Hence, the controller needs to observe the SCR state in both $\mathrm{NH}_{3}$ and NOx slippage and provide a clear output for engine to operate lean air-fuel ratio. MATLAB's inbuilt fuzzy logic toolbox was used in order to design and setup the control features of the fuzzy-logic algorithm (Mathworks 2016).

Under the boundaries of the above-mentioned objectives, the fuzzy-logic controller was designed taking into consideration three input parameters; (Input-1) Ammonia Slip \{AS\}, (Input-2) Rate of Ammonia Slip $\{$ RAS $\}$, and (Input-3) NOx Slip $\{$ NOS $\}$. AS and NOS inputs are obtained from direct measurement, whereas, the ASR is computed by taking the difference of ammonia slip concentration between the $\mathrm{NH}_{3}$ concentration at $(\mathrm{t})$ second and $(\mathrm{t}-\mathrm{n})$ second 
in the continuous data. The " $n$ " would be defined from observing the data's response to avoid high sudden peaks attributed due to signal noise. The output of the developed controller will, hence, define the engine AFR status to either be on lean-ON status $\left(\mathrm{NH}_{3}\right.$ regeneration) or leanOFF status (baseline operation).

The fuzzy-logic based controller consists of three main processes; fuzzification, inferences, and de-fuzzification. Figure 24 provides the flow-chart of the fuzzy-logic's decisionmaking algorithm design for the study, and their detailed steps of the processes are discussed in the sections below. The fundamentals and the approach used in developing the fuzzy control

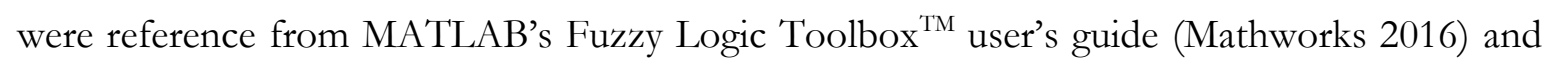
handouts provided by Dr. Mario Perhinschi's MAE565 AI Techniques in MAE class at WVU (Perhinschi 2016). Snippets of MATLAB's Fuzzy Logic Toolbox ${ }^{\mathrm{TM}}$ used in designing the fuzzy logic controller are provided in APPENDIX B.

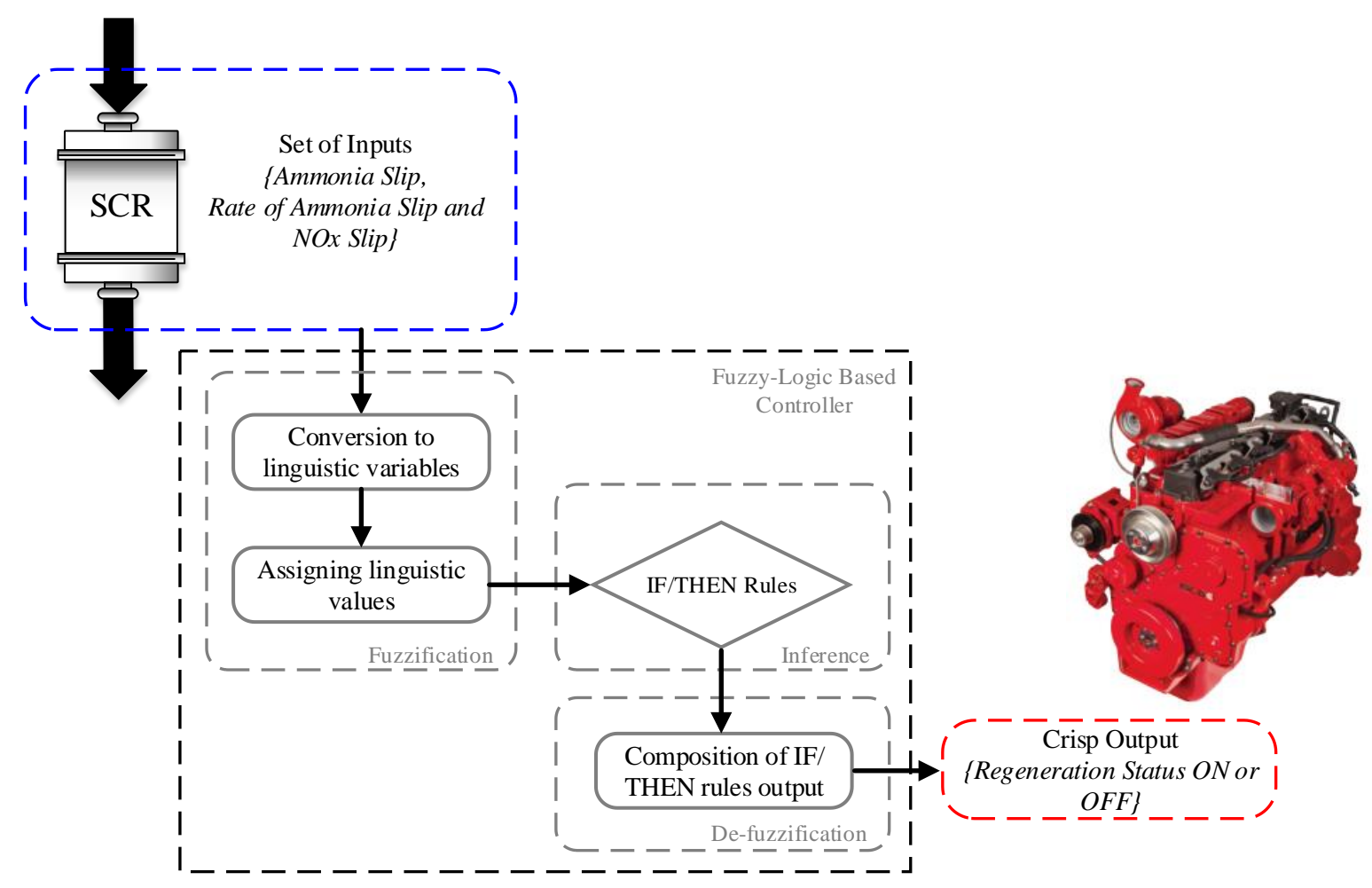

Figure 24. Flow-chart showing the fuzzy-logic based controller for controlling the passive-ARC regeneration status 


\subsubsection{Fuzzification}

Fuzzification is a process which takes crisp input from the input interface and converts it into a fuzzy input sets. The three inputs (AS, RAS and NOS), also named as linguistic variables in the fuzzy-logic terminology, are derived into three input domains having its own fuzzy sets, that is defining the linguistic values for each linguistic variable and is provided in Table 3 . The linguistic values represent the nature of the linguistic variables and degree to which the crisp measurements belong to each linguistic value is defined by membership function, as discussed in the next section.

Table 3. List of linguistic variables and linguistic values

\begin{tabular}{|c|c|c|c|}
\hline \multicolumn{2}{|l|}{ Linguistic Variables } & \multicolumn{2}{|c|}{ Linguistic Values } \\
\hline \multirow{4}{*}{ Ammonia Slip } & \multirow{4}{*}{ (AS) } & Negligible Slip & (NS) \\
\hline & & Small Slip & $(\mathrm{SS})$ \\
\hline & & Moderate Slip & (MS) \\
\hline & & Large Slip & (LS) \\
\hline \multirow{3}{*}{ Rate of Ammonia of Slip } & \multirow{3}{*}{ (RAS) } & Increasing & (IR) \\
\hline & & Constant & (CR) \\
\hline & & Decreasing & (DR) \\
\hline \multirow{4}{*}{ NOx Slip } & \multirow{4}{*}{ (NOS) } & Negligible Slip & $(\mathrm{NS})$ \\
\hline & & Small Slip & (SS) \\
\hline & & Moderate Slip & (MS) \\
\hline & & Large Slip & (LS) \\
\hline
\end{tabular}

\subsubsection{Membership function}

In fuzzy-logic control theory, the membership function is designed to represent the extent or degree to which a value of a linguistic variable belongs to a fuzzy set, i.e. linguistic values. This maps the three input variables given in its respective membership function of the linguistic values in relationship with the desired output. The shape of the membership function is designed based on arbitrary numbers which are placed based on data range of the three selected inputs. For this study, the values in approximating the linguistic values in shaping the membership function is only obtained after conducting the SCR evaluation. The study elects to utilize trapezoidal shaped membership function for the input linguistic variables. With the trapezoidal shape, the end fuzzy sets of the linguistic values are shaped based on increasing and decreasing type functions. Figure 25 depicts the shape of the membership function of the input variables, indicating the trapezoidal functions with increasing and decreasing types and the degree of overlap between the functions. On the other hand, the output function was given a 
triangular shaped membership function. The output variable determines the regeneration status, which is "Lean-ON" or "Regen-OFF", and dictates a distinct mode to be on either of these statuses. The lean-ON status was further defined to be in two separate modes, based on dithering and defined as short-dithering (SD) and long-dithering (LD) as represented in Figure 26. As an engine-based control strategy to regenerate the passive-ARC system, the lean-ON is set to a fixed dithering time instead of a feedback-loop based control strategy. For example, in such event, the engine is continuously operated for a given time and the length of lean-period is given in two types (short-dithering and long-dithering). This would potentially benefit in transient operation which consist of hard-acceleration events, causing sudden emission peaks and rise-rates. Additionally, this could also avoid potential control fluctuations in the controlling the engine operation which are known to have response delays at different load demands.

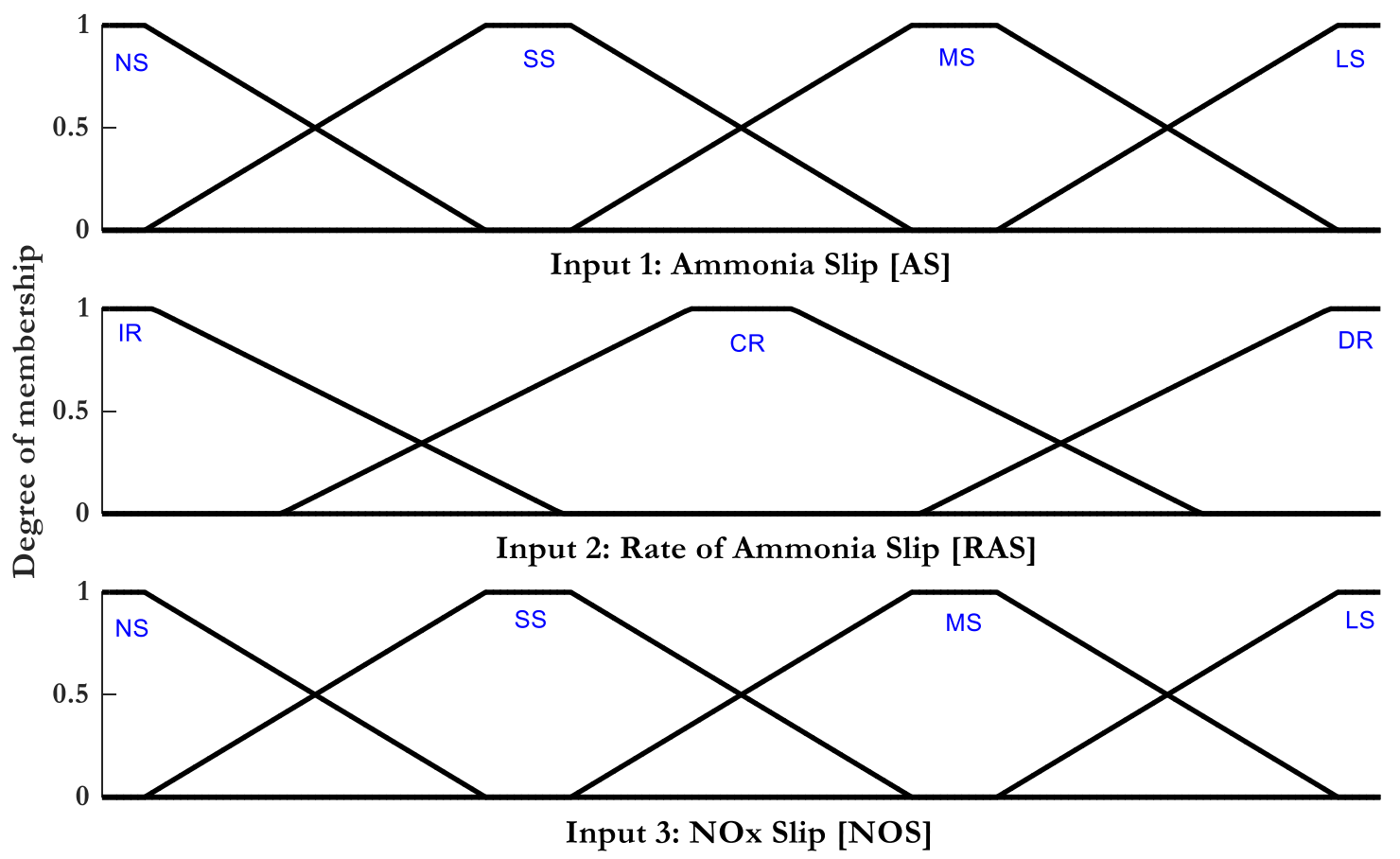

Figure 25. Membership function shapes for three input linguistic variables and its values 


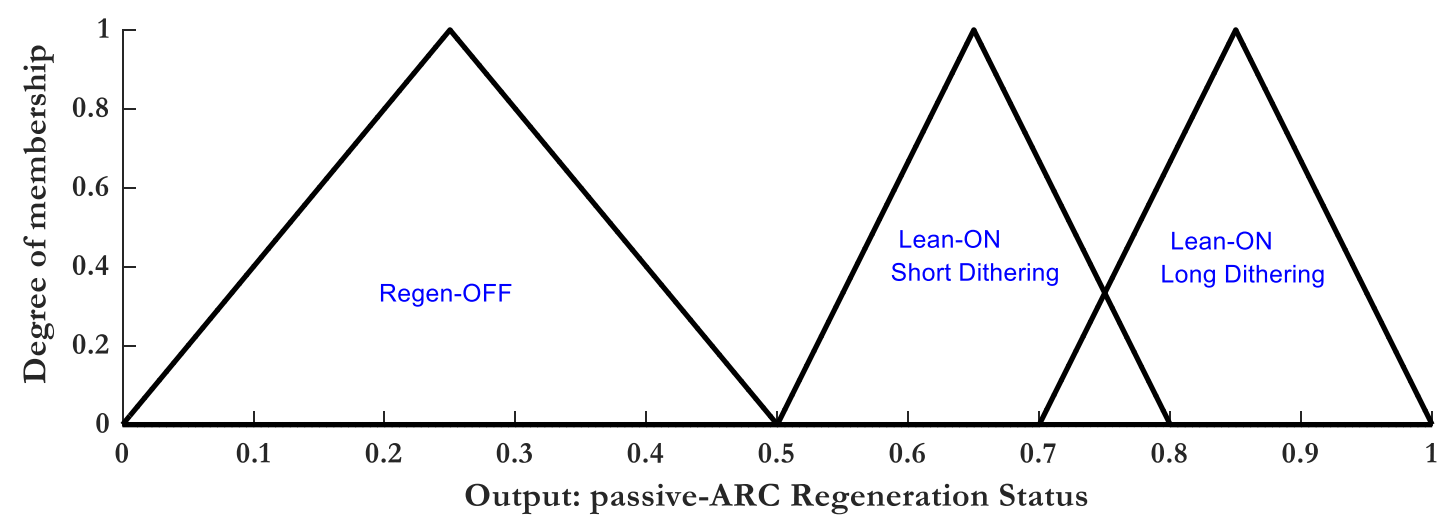

Figure 26. Membership function for output variable

\subsubsection{Inference engine}

The inference engine of the decision-making controller generates the fuzzy commands based on the inference rules defined in the design process using some understanding of the after-treatment behavior. In considering the three linguistic variables and combination of (4, 3 and 4) levels of linguistic values for each linguistic variable, resulted in a total of 48 inference rules that defined the regeneration status. All 48 input-output combinations of rules are provided in Figure 27. Below are some examples of the decision statements ("IF-THEN" rules) used in determining the conditions in the inference rules with respect to the three-linguistic variable and its linguistic values to the three the outputs:

Example Rule: If ammonia slip (AS) is negligible slip (NS) and rate of ammonia is slip (RAS) is increasing rate (IR) and NOx slip is negligible (NS), then regen status is off (Off)

Example Rule: If ammonia slip (AS) is large slip (LS) and rate of ammonia is slip (RAS) is increasing rate (IR) and NOx slip is negligible (NS), then regen status is on and large dithering (On-LD)

Example Rule: If ammonia slip (AS) is small slip (SS) and rate of ammonia is slip (RAS) is increasing rate (IR) and NOx slip is small slip (SS), then regen status is on and small dithering (On-SD) 


\begin{tabular}{|c|c|c|c|c|c|}
\hline IR & On-SD & On-LD & On-LD & On-LD & \multirow{3}{*}{ NS } \\
\hline CR & On-SD & On-SD & On-LD & On-LD & \\
\hline DR & Off & On-SD & On-LD & On-LD & \\
\hline IR & Off & On-SD & On-LD & On-LD & \multirow{3}{*}{ SS } \\
\hline CR & Off & On-SD & On-SD & On-SD & \\
\hline DR & Off & Off & On-SD & On-SD & \\
\hline IR & Off & Off & Off & Off & \multirow{3}{*}{ MS } \\
\hline CR & Off & Off & Off & Off & \\
\hline DR & Off & Off & Off & Off & \\
\hline IR & Off & Off & Off & Off & \multirow{3}{*}{ LS } \\
\hline $\mathrm{CR}$ & Off & Off & Off & Off & \\
\hline DR & Off & Off & Off & Off & \\
\hline & NS & SS & MS & LS & \\
\hline
\end{tabular}

Ammonia Slip (AS)

Figure 27. 48 inference rule based matrix for fuzzy-logic output

Individual-rule based inference method was used for rule firing, to obtain the fuzzy commands using the inference rule matrix. For given number of three inputs, we get three sets of fuzzy input and using a composition rule method will provide in obtaining the membership value associated to the intersection of the fuzzy set to the inputs. From fuzzy methodology of logical operators in fuzzy sets, three definitions were considered for computing the intersection region of the fuzzy set and are given by the relationships below:

i. Multiplicative definition:

$$
V_{1} \cap V_{2} \cap V_{3}=\left\{x \mid \mu_{V_{1} \cap V_{2} \cap V_{3}}(x)=\mu_{V_{1}}(x) \cdot \mu_{V_{2}}(x) \cdot \mu_{V_{3}}(x)\right\}
$$

ii. Minimum definition:

$$
V_{1} \cap V_{2} \cap V_{3}=\left\{x \mid \mu_{V_{1} \cap V_{2} \cap V_{3}}(x)=\min \left[\mu_{V_{1}}(x), \mu_{V_{2}}(x), \mu_{V_{3}}(x)\right]\right\}
$$

iii. Additive definition:

$$
V_{1} \cap V_{2} \cap V_{3}=\left\{x \mid \mu_{V_{1} \cap V_{2} \cap V_{3}}(x)=\mu_{V_{1}}(x)+\mu_{V_{2}}(x)+\mu_{V_{3}}(x)\right\}
$$

The multiplicative and the minimum are the two most commonly used definitions of intersection for control purposes, the minimum fuzzy set intersection method is the one utilized for this study. 


\subsubsection{De-fuzzification}

De-fuzzification, considered as the output interface, is the process of converting the fuzzy commands into user defined crisp values, i.e. the regeneration status with command basic command logic (On/Off) that can be executed by the ECU interface module. The uses the use of fuzzification of clipped or scaled membership function for the output linguistic variables. The study used "the middle of maximum" or basically the average of the maximum value of the output set as the De-fuzzification method, due to the fact the only three statuses is executed by the fuzzy output interface. The relationship of computing the states is given below by Equation (12) and Equation (13). This entails the center of area under the curve from the aggregated output of all the clipped fuzzy sets as depicted in Figure 28 and center of the maximum area is selected as the regen status (example indicating fuzzy output of 0.25 with crisp output of Regen$\mathrm{OFF})$.

$$
\begin{gathered}
u^{*}=c_{i \max , j \max } \\
d_{i \max , j \max }=\max _{i, j}\left(d_{i j}\right)
\end{gathered}
$$

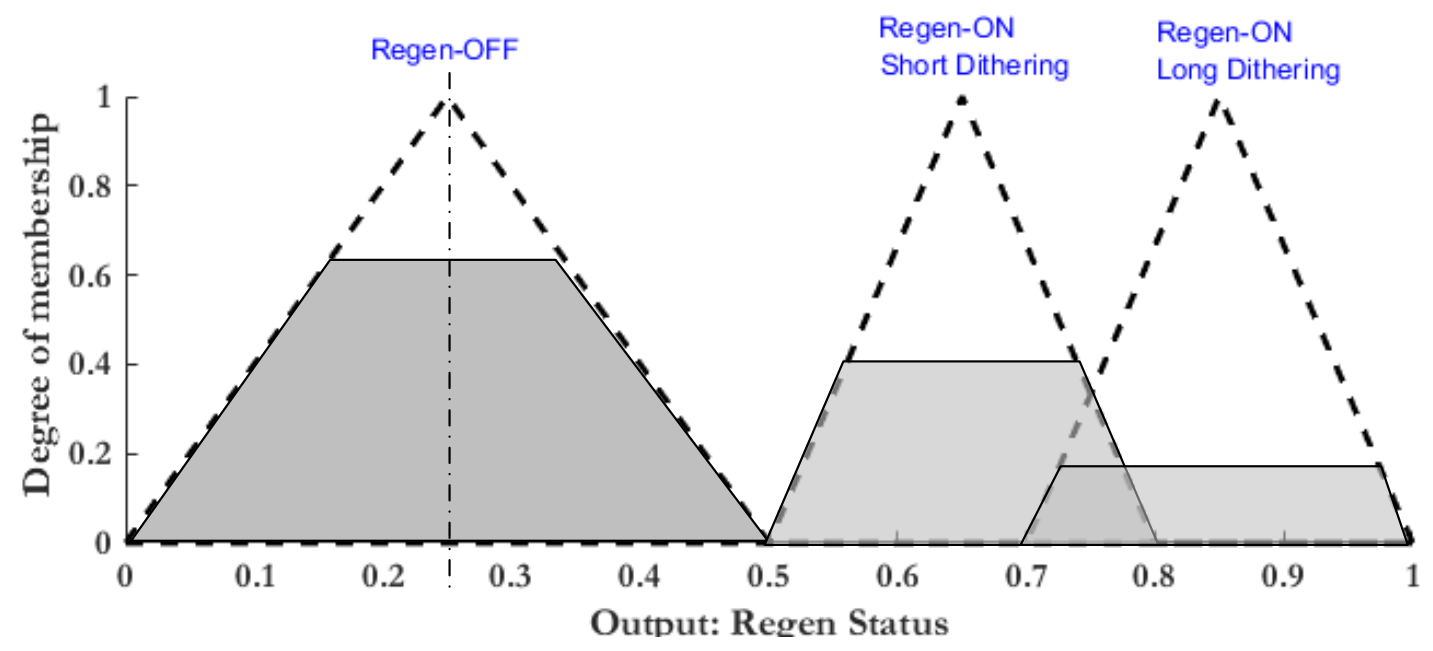

Figure 28. De-fuzzification of the fuzzy-logic output using middle of maximum method 


\subsubsection{Fuzzy logic control summary}

In summary, the control algorithm approached consists of fuzzy-logic inferenced logic features specifically designed to command engine based SCR regeneration using feedback from only two sensor outputs, i.e. $\mathrm{NOx}$ and $\mathrm{NH}_{3}$ values after the SCR. Linguistic values of the fuzzylogic sets will be based on experimental profiles obtained from transient and steady-state test conducted during the selected SCR evaluation. The analysis of this approach will focus on validating closed-loop $\mathrm{NH}_{3}$ slip control along with meeting low $\mathrm{NOx}$ emission requirement. Different regeneration duration (amount of time the engine is operated at lean AFR condition) along with transient compensations will be looked at in order to understand the engine behavior and the after-treatment response.

The fuzzy logic controller can also be designed with incorporating additional input variables such as temperature and ammonia storage ratio for wider control of the regen events even in the situation where there is no noticeable amount of ammonia slip with low levels of NOx trace. This can be performed by monitoring the SCR's state with estimation of high ammonia storage conditions and brief lean-ON trigger can be initiated further extending the control strategy under wider range of engine operation. Such approach could possibly provide feasible robust control of a real time on-board $\mathrm{NH}_{3}$ generation and regeneration strategy. Additionally, possibility of expanding the overall range of natural gas engine operation beyond its tight stoichiometric regime to more lean burn operation. This leads into the work of modebased approach as discussed in the follow sections. 


\subsection{Model-based estimator methods}

The intent of the following approach is to evaluate proposed after-treatment activity models for estimating the state of the passive-ARC system, mainly in estimating $\mathrm{NH}_{3}$ storage ratio along and $\mathrm{NH}_{3}$ concentration values outlet of the passive-ARC system. The study investigates into evaluating after-treatment activity models, which can also be used as real-time in-ECU basis for predicting $\mathrm{NH}_{3}$ concentration before and after the SCR system based on various engine and after-treatment parameters.

Figure 29 illustrates the modeling schematic, depicting the two after-treatment models (Model-1 and Model-2) with its input parameters (X1 to X7 shown by red arrows) and the output parameters (Y1, Y2 and Y3) shown by the blue arrows). The Model-1 includes engine plus the TWC as one combined system and estimates $\mathrm{NH}_{3}$ concentration post-TWC using eight different engine and after-treatment parameters as model inputs. Whereas, Model-2 includes only the SCR system model which will use pre-and-post SCR parameters, estimating post-SCR $\mathrm{NH}_{3}$ slippage as the model output (Y2). Although not considered in the fuzzy-logic control inputs, the study will also focus on estimating the $\mathrm{NH}_{3}$ storage ratio of the SCR catalyst. The estimated $\mathrm{NH}_{3}$ (Y1) from the proposed Model-1 output is also used as input variable for Model2. The focus of this approach is to develop an SCR catalyst model which can be implemented as a control-oriented model.

The study considers identifying major SCR kinetics that governs the characteristics of reactions in both gas and solid phase species for $\mathrm{NOx}$ and $\mathrm{NH}_{3}$. Higher order $\mathrm{SCR}$ models have shown to represent SCR systems dynamics accurately and has helped in the better control of the urea-injection process in diesel applications. However, such approach in the vehicle application could be computationally intensive, leading to impracticality in on-ECU real time implementation. Therefore, these studies utilize reduce order models that were necessary to identify the dynamic behavior of the SCR and was adequate to estimate model parameters. The approach towards estimating $\mathrm{NO}$ and $\mathrm{NH}_{3}$ after the SCR is carried with step-by-step identification of physical and chemical processes of species entering the SCR catalyst. From the basic principle of the SCR operation, it was necessary to formulate model that uses mechanism of ammonia of adsorption/desorption, reduction and oxidation of $\mathrm{NO}$ and $\mathrm{NH}_{3}$ over the selected SCR catalyst. 
INPUTS

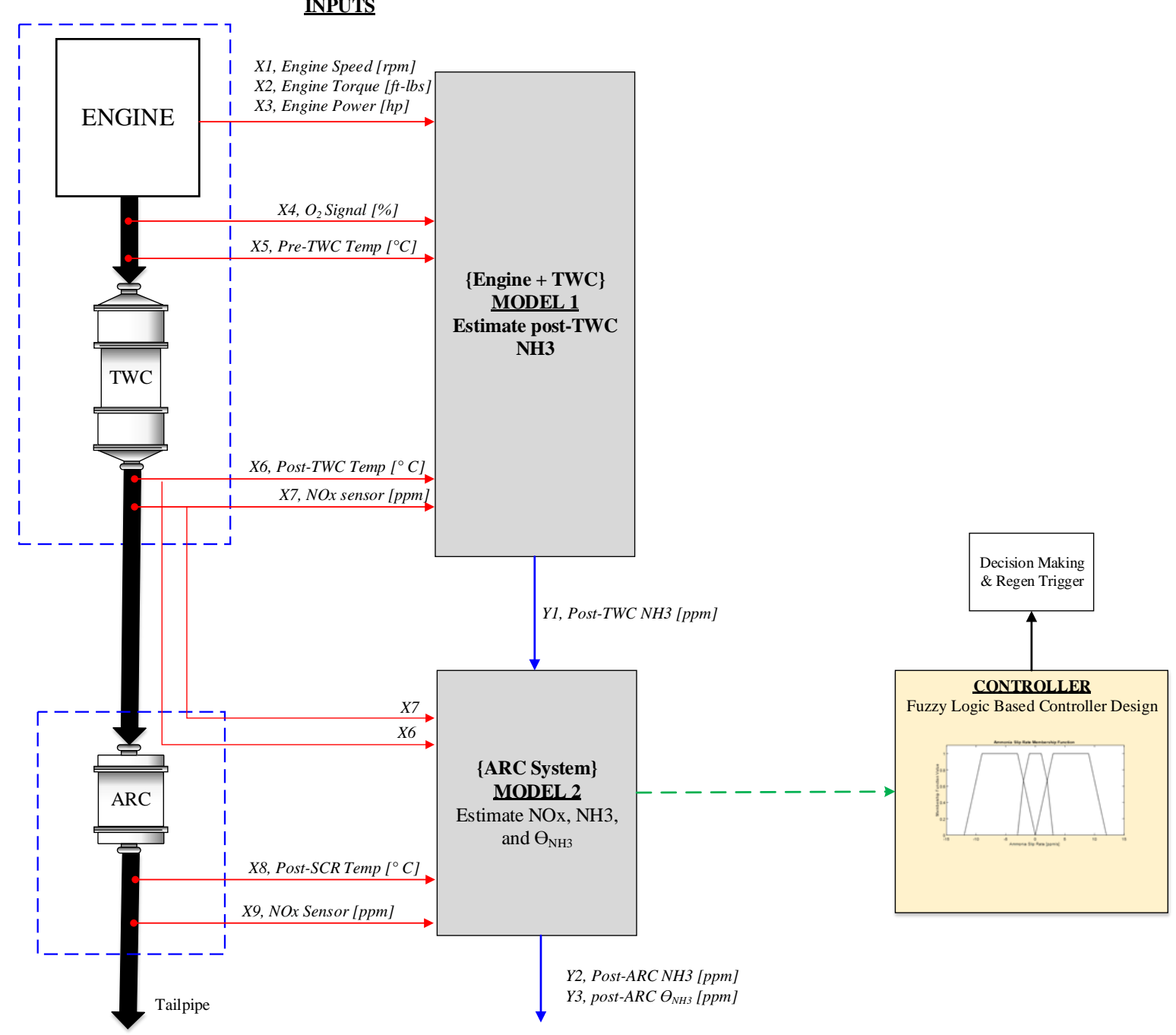

Figure 29. Schematic of model-based $\mathrm{NH}_{3}$ estimation approach for the fuzzy-logic based controller

\subsubsection{Post-TWC $\mathrm{NH}_{3}$ estimation method}

Model 1 utilizes a data driven artificial neural-network (ANN) fitting methodology to map the selected seven input parameters to estimate the $\mathrm{NH}_{3}$ concentration after the TWC system. The ANN methods are known to be a good predictor when there is not enough information to describe the functional form of a system response or the relationship between the inputs and the output (De Cesare and Covassin 2011). Therefore, ANN has been a viable tool and approach using data driven modeling in adequately predicting certain system output even without understanding the exact mathematical and physical aspects of a system in relation to factors influencing its complex behavior. Use of such techniques were formed from the notion of how human brain's networking architecture operates. Like multiple number of 
neurons in the brain transmitting information efficiently within different networks, the basic architecture of ANN does consist of similar functional layers (analog to neurons and networks) which process information with multiple inputs and outputs. Within such layers, there could be multiple nodes or neurons and each neuron receives information from respective inputs or from other neurons through networks or paths. And these paths are then modified with computed weights through leaning process to achieve desirable outputs. In similar note, it approximates arbitrary continuous functions with certain degree of accuracy, if the sets of dependent and independent variables are known with adequate information of input and output data set (Prié et al. 2015). The network of inputs-neurons-outputs or connecting input to output with hidden layers of pathway are modeled with defined activation functions and the model can be used in predicting the future behavior of the same system with certain accuracy.

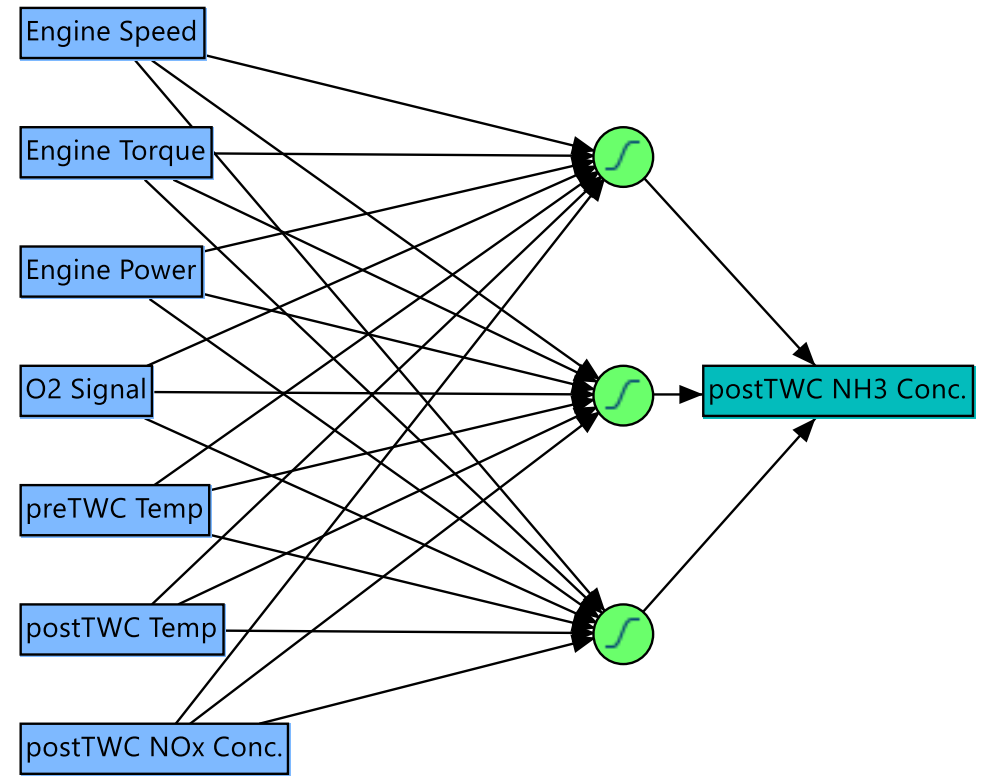

\section{Figure 30. Example flow model architecture of ANN with seven inputs, three hidden layers and one output}

The selection of the seven input parameters were founded on the basic of influential factors in characterizing the after-treatment system based on similar research studies and open literatures. Figure 30 depicts the flow model of the neural-network (N-N) setup showing an example of a network with connecting seven inputs with the single output using three hidden layers. The study does realize that there could be additional high-impacting factors as input 
parameters in the model which could potentially help in better estimating $\mathrm{NH}_{3}$ concentration, but due to limited availability in ECU parameters, the study utilizes the readily available channels and evaluates the accuracy level of prediction appropriate in using as an on-board control feedback approach. Towards on-ECU implementation standpoint, it also becomes extremely important in understanding the computational time utilizing such models and processing in realtime application.

For the purpose of this study, a two-layer feed-forward function fitting network using MATLAB $^{\circledR}$ 's inbuilt ANN toolbox was used in evaluating and identifying different training algorithms and appropriate network connection. Utilizing MATLAB's step-by-step approach helped to create, train and visualize models. The overview of the network flow obtained from MATLAB $^{\circledR}$ s s net is provided by Figure 31. The performance of the N-N architecture to fit the target with the given sets of inputs depends on different network parameters. Based on work conducted in the area of $\mathrm{N}-\mathrm{N}$, the two major parameters that tend to alter the network performance is the size of the hidden layers and pre-defined training functions used.

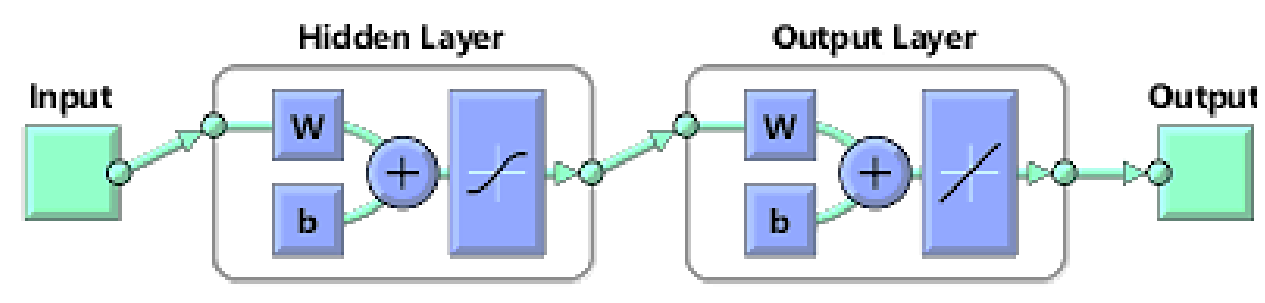

Figure 31. MATLAB's Neural-Network view

The study looks into evaluating different feed-forward based network architecture to achieve improvements in training, if any, in the output estimation i.e. concentration of $\mathrm{NH}_{3}$ being produced by the TWC during transient and steady-state operation of the engine. The two types of training algorithm used to evaluate the network are the Levenberg-Marquardt and Bayesian Regularization functions. The first method, Leavenberg-Marquardt uses a backpropagation (of errors) based algorithm to train and optimize weights and bias with minimizing sum of square error (Mathworks 2017). Similarly, the Bayesian regularization is built on using the Levenberg-Marquardt algorithm to optimize weight and bias values but additionally uses error minimization technique using linear combination of squared errors and weights. Due to the additional training computation in Bayesian Regularization backpropagation technique, 
Levenberg-Marquardt is typically considered as a faster approach in generalizing networks. The summary of the configuration chosen for evaluating the training of the $\mathrm{N}-\mathrm{N}$ are given Table 4. For the training of the models, the input-output data set is selected to be divided into three subsets, training, validation and testing as also provided in the table. The present of the training data set is used for computing the gradient and updating the network weights and biases, whereas, the validation set is used for monitoring the error during the training process. It essentially helps in understanding and avoiding potential overfitting of the data, if any during the event of training and validating the input-output relationships. These are observed from the mean-square error plots, where high deviation in the error between the training set and the validation set starts to increase in differing paths. On the other hand, the third division, which is the testing set's error is not used during the training events and can be used to evaluate against different trained models and data set. All the N-N properties in terms of training the model and input-out fitting performance and results, will be evaluated in terms of computational time.

\section{Table 4. Neural-Network Training Parameters}

\begin{tabular}{|l|l|}
\hline Number of Inputs & 7 \\
\hline Size of Hidden Layers & {$[10,20,30,40]$} \\
\hline Training Functions & {$[$ Levenberg-Marquardt, Bayesian Regularization $]$} \\
\hline Data Set Division & Training $=70 \% ;$ Validation $=15 \% ;$ Testing $=15 \%$ \\
\hline
\end{tabular}

As for the input-output data set, a transient FTP test is used to train the model initially with the seven selected input parameters to estimate the transient $\mathrm{NH}_{3}$ production after the TWC. As the computational time of neural networks are known to increase with using higher number of input parameters, the study also considers examining the cross-correlation between the input and output parameters to eliminate the number of inputs in the overall modelling approach.

\subsubsection{Post-SCR $\mathrm{NH}_{3}$ estimation method}

Model 2 utilizes a control oriented modeling approach steered more towards a modelbased online estimation application. Due to dynamics of SCR system in $\mathrm{NH}_{3}$ storage and reaction, it becomes crucial to better understand kinetics of SCR over the transient behavior of the exhaust with which it gets introduced. The study approaches $\mathrm{NH}_{3}$ estimation after the SCR catalyst from a model-based method with utilizing kinetic models over the chosen SCR catalyst. 
From the obtained literatures as discussed SCR formulation review section system, various reactions mechanism is important in order to describe the true behavior of the SCR in outputting certain levels of $\mathrm{NOx}$ and $\mathrm{NH}_{3}$. Hence, identifying the adsorption and desorption response of the $\mathrm{NH}_{3}$ over the catalyst surface is vital in capturing the dynamic behavior of the system in different operating condition. The SCR system are known to be highly non-linear in nature and the internal mechanism operates in different time scale along with its operation being strictly varied due to changing temperatures in the exhaust. The temperature strongly influences the rate of chemical reactions are known to depend exponential on temperature (Figura et al. 2016). From kinetic model approach, the follow section discusses the estimation of concentration and coverage ratio of ammonia over the added SCR system for the study.

\subsubsection{SCR kinetic analysis}

The ammonia entering the SCR system gets adsorbed and desorbed by the SCR substrate and the two-way adsorption and desorption of the ammonia reaction is given by the following expression (M. F. Hsieh and Junmin Wang 2010):

$$
\mathrm{NH}_{3}+\theta_{\text {free }} \rightarrow \mathrm{NH}_{3}^{*}
$$

where, $\theta_{\text {free }}$ represents the surface that is not covered by the $\mathrm{NH}_{3}$ and has potential for ammonia adsorption. The rate of reactions of the following above for adsorption/desorption processes can be modeled using the Arrhenius equations and presented below:

$$
\begin{gathered}
R_{a d s}=k_{a d s} \exp \left(-\frac{E_{a d s}}{R T}\right) C_{N H 3}\left(1-\theta_{N H 3}\right) \Omega \\
R_{d e s}=k_{d e s} \exp \left(-\frac{E_{d e s}}{R T}\right) \theta_{N H 3} \Omega
\end{gathered}
$$

where, $R_{a d s}$ and $R_{\text {des }}$ are the two reaction rates for adsorption and desorption reactions (in mole/s), respectively. $\mathrm{T}$ is the temperature in $\mathrm{K}, \mathrm{C}_{\mathrm{NH} 3}$ represent the model concentration of ammonia in mole $/ \mathrm{m}^{3}, E_{a d s} \& E_{\text {des }}$ are the activation energy and $\mathrm{k}_{\mathrm{ads}} \& \mathrm{k}_{\text {des }}$ are the rate constant from the Arrhenius reaction models. $\theta_{N H 3}$ are the ammonia coverage ratio which is defined by Equation (17), where $M_{N H 3}^{*}$ represents the mode number of ammonia absorbed by the catalyst 
and $\Omega$ is the ammonia storage capacity of the catalyst in moles and which tend to vary with temperature and is typically modeled by Equation (17), where $S_{1}$ and $S_{2}$ are the storage parameters and positive constants. The ammonia coverage ratio goes from 0 to 1 , where 0 stands for no ammonia stored and 1 stands for saturated SCR brick. V is the catalyst volume expressed in $\mathrm{m}^{3}$.However, these parameters needs to change accordingly to capture the effect of catalyst aging as an adaptive approach (M. F. Hsieh and Junmin Wang 2010).

$$
\begin{gathered}
\theta_{N H 3}=\frac{M_{N H 3}^{*}}{\Omega} \\
\Omega=S_{1} e^{-S_{2} T}
\end{gathered}
$$

The adsorbed ammonia is either desorbed as described from above mechanism or further reacts with NOx as by the process of reduction reaction. The standard NOx reduction reaction, as discussed in the literature reviewed Section 15, is the dominant reaction due to presence of only NO in the exhaust. Reiterating the reaction (8) with only the adsorbed ammonia $\mathrm{NH}_{3}^{*}$, Equation (19) expresses the reaction between the stored $\mathrm{NH}_{3}$ over the SCR surface and exhaust $\mathrm{NO}$ species in the presence of $\mathrm{O}_{2}$. The rate of reaction for the following NOx reaction is ones again defined from the Arrhenius reaction rates provided by Equation (20), where $C_{N O}$ and $C_{O_{2}}$ represents the mole concentration of $\mathrm{NO}$ and $\mathrm{O}_{2}$, respectively. All the reaction rates were defined as a function stored ammonia $\left(\theta_{N H 3}\right)$ and gaseous NO.

$$
\begin{array}{r}
4 \mathrm{NH}_{3}^{*}+4 \mathrm{NO}+\mathrm{O}_{2} \rightarrow 4 \mathrm{~N}_{2}+6 \mathrm{H}_{2} \mathrm{O} \\
R_{\text {red }}=k_{\text {red }} \exp \left(-\frac{E_{\text {red }}}{R T}\right) C_{\mathrm{NO}} C_{\mathrm{O}_{2}} \theta_{\mathrm{NH} 3} \Omega
\end{array}
$$

Additionally, the adsorbed $\mathrm{NH}_{3}^{*}$ also has the tendency to get oxidize in the presence of $\mathrm{O}_{2}$ as expression by reaction (21). Such reactions are typically known to occur at temperatures higher than $450^{\circ} \mathrm{C}$ (Jinbiao and Fengjun 2015). The reaction rate of the $\mathrm{NH}_{3}$ oxidation is 
provided by Equation (22), where $k_{\text {oxi }}$ is the rate constant for oxidation and $E_{\text {oxi }}$ is the activation energy for the oxidation process.

$$
\begin{array}{r}
4 \mathrm{NH}_{3}^{*}+3 \mathrm{O}_{2} \rightarrow 2 \mathrm{~N}_{2}+6 \mathrm{H}_{2} \mathrm{O} \\
R_{\text {oxi }}=k_{\text {oxi }} \exp \left(-\frac{E_{\text {oxi }}}{R T}\right) C_{O_{2}} \theta_{N H 3} \Omega
\end{array}
$$

\subsubsection{Steady-state estimator for SCR modeling}

For solving the reaction mechanisms, the study assumes the SCR catalyst to be a continuous stirred tank reactor (CSTR) in order to develop is simplified 0-D model (M. F. Hsieh and Wang 2011). This assumes the SCR to be a single unit reactor with the states being homogenous within the catalyst as depicted in Figure 32.

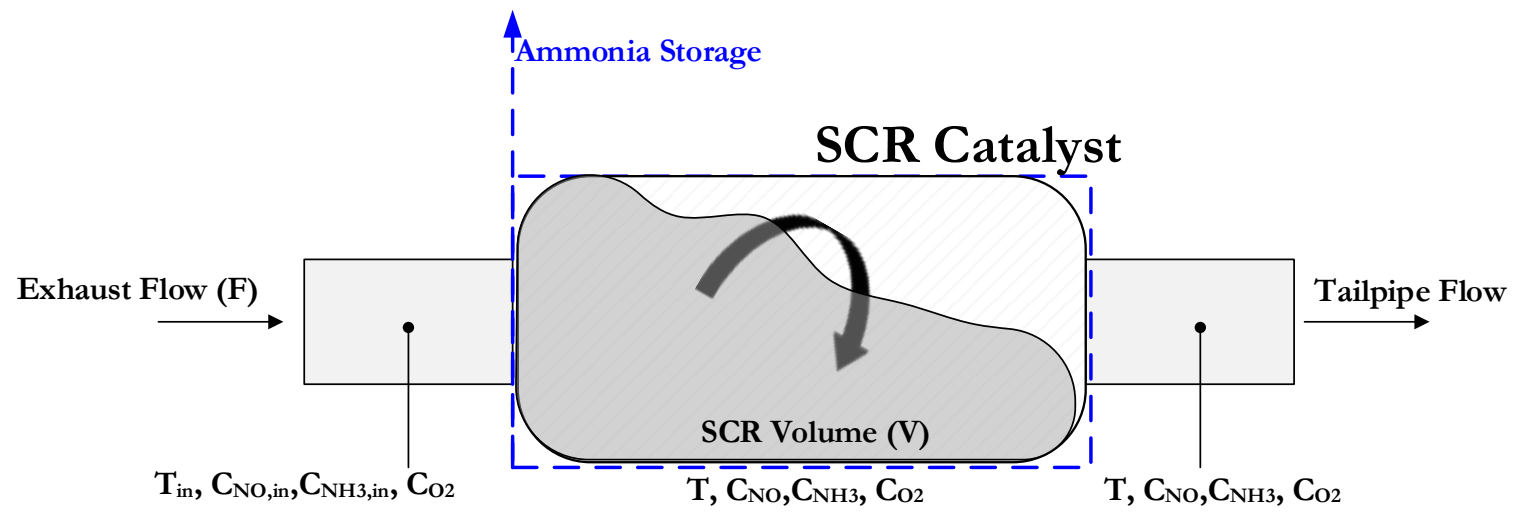

Figure 32. Schematic of ammonia storage distribution and CSTR model of SCR catalyst

In additional to the main assumption, the following assumptions were also made to formulate the SCR model and are listed below (Devarakonda, Parker, and Johnson 2008):

1. All chemical kinetics over the catalyst surface are reaction control with no mass transfer.

2. Surface phase concentration of varied species are neglected except for $\mathrm{NH}_{3}$ storage

3. NOx reaction with $\mathrm{NH}_{3}$ is assumed to be with $\mathrm{NO}$ only

4. Reaction rate are defined as a function of SCR inlet temperature, $\mathrm{NH}_{3}$ storage ratio and concentration of $\mathrm{NO}$ at the SCR inlet. 
Using the conservation of mass balance law and the above mentioned CSTR assumptions, the three state model equations are expressed by the following three equations below.

$$
\begin{gathered}
\dot{C}_{N O}=\bar{Q}\left(C_{N O, \text { in }}-C_{N O}\right)-R_{\text {red }} \\
\dot{\theta}_{N H 3}=R_{a d s}-R_{\text {des }}-R_{\text {red }}-R_{\text {oxi }} \\
\dot{C}_{N H 3}=\bar{Q}\left(C_{N H 3, \text { in }}-C_{N H 3}\right)+R_{\text {des }}-R_{\text {ads }}
\end{gathered}
$$

where $\bar{Q}$ is the normalized exhaust flow rate $(1 /$ s) obtained from volumetric exhaust flowrate (F) divided by total catalyst gas volume $(\mathrm{V})$. The $C_{N O \text {,in }}$ and $C_{N H 3, i n}$, are $\mathrm{NO}$ and $\mathrm{NH}_{3}$ concentration upstream of the catalyst. The above equations are rearranged to a state-space model form which are shown in Equation (26).

$$
\left[\begin{array}{c}
\dot{\mathrm{C}}_{\mathrm{NO}} \\
\dot{\theta}_{\mathrm{NH} 3} \\
\dot{\mathrm{C}}_{\mathrm{NH} 3}
\end{array}\right]=\left[\begin{array}{c}
-\mathrm{r}_{\mathrm{red}} \mathrm{C}_{\mathrm{NO}} \mathrm{C}_{\mathrm{O} 2} \theta_{\mathrm{NH} 3} \Omega-\bar{Q} \mathrm{C}_{\mathrm{NO}} \\
-\theta_{\mathrm{NH} 3}\left(\mathrm{r}_{\mathrm{ads}} \mathrm{C}_{\mathrm{NH} 3}+\mathrm{r}_{\mathrm{oxd}} \mathrm{C}_{\mathrm{O} 2}+\mathrm{r}_{\mathrm{des}} \mathrm{C}_{\mathrm{NO}} \mathrm{C}_{\mathrm{O} 2}\right)+\mathrm{r}_{\mathrm{ads}} \mathrm{C}_{\mathrm{NH} 3} \\
-\mathrm{C}_{\mathrm{NH} 3}\left(\Omega \mathrm{r}_{\mathrm{abs}}\left(1-\theta_{\mathrm{NH} 3}\right)+\bar{Q}\right)+\mathrm{r}_{\mathrm{des}} \Omega \theta_{\mathrm{NH} 3}
\end{array}\right]+\left[\begin{array}{c}
0 \\
0 \\
\bar{Q}
\end{array}\right] \mathrm{C}_{\mathrm{NH} 3, \mathrm{in}}+\left[\begin{array}{c}
\bar{Q} \\
0 \\
0
\end{array}\right] \mathrm{C}_{\mathrm{NO}, \mathrm{in}}
$$

where $r_{i}=k_{i} \exp \left(-\frac{E_{i}}{R T}\right), i=a d s$, des, red, oxi

The study incorporates the above mentioned three SCR mechanisms to be considered in the control-oriented SCR model. The state coupled SCR equation model is a system of firstorder Ordinary Differential Equations (ODEs) which includes rate of reaction of dependent variables $\mathrm{NO}$ and $\mathrm{NH}_{3}$ along ammonia storage ratio. The study used differential equation solvers using MATLAB's ODE15s method to solve the system of kinetic equations, simultaneously (MathWorks 2017). The solver numerically solves using numerical differentiation formulas (NDF's) of orders 1 to 5. The time derivative reaction rate was formulated as objective function in MATAB workspace to solve the ODE problem with given initial values of $\mathrm{NO}, \mathrm{NH}_{3}$ and $\dot{\theta}_{\mathrm{NH} 3}$. 
For the model to estimate the SCR reaction outcomes, the reaction parameters $\left(k_{i}\right.$ and $\mathrm{E}_{\mathrm{i}}$ ) in the reaction mechanisms needs to be given, and the values are usually obtained from conducting controlled reactor experiments and was out of the scope of the study. There exist several literature that have looked at identifying the reaction rate parameters based on lab reactor experiments conducted for similar SCR systems (Clark et al. 2009; Song 2013; Pant and Schmieg 2011). However, studies have also shown using optimization or search based techniques for parameter identification (M. F. Hsieh and Wang 2011; Devarakonda, Parker, and Johnson 2008; Song et al. 2013). The approach taken in this study is discussed in the following section by solving the ODE model with different combination of parameters and then comparing the obtained model values with the actual measured values.

\subsubsection{Model parameter identification method}

The approach uses MATLAB $^{\circledR}$ 's multi-objective Genetic Algorithm (GA) method to obtained the reaction parameters by solving the reactor model with combination of varying values of the parameters to be optimized. The multi-objective optimization technique is used in minimizing multiple objective functions which are subject to set of constraints. Likewise, the model consists of three state variables as objective function that needs to be optimized for the given set of parameters, i.e. activation energies and rate constant for each reaction. Figure 33 presents the parameter identification schematic formulated for the GA based optimization problem in MATLAB ${ }^{\circledR}$.

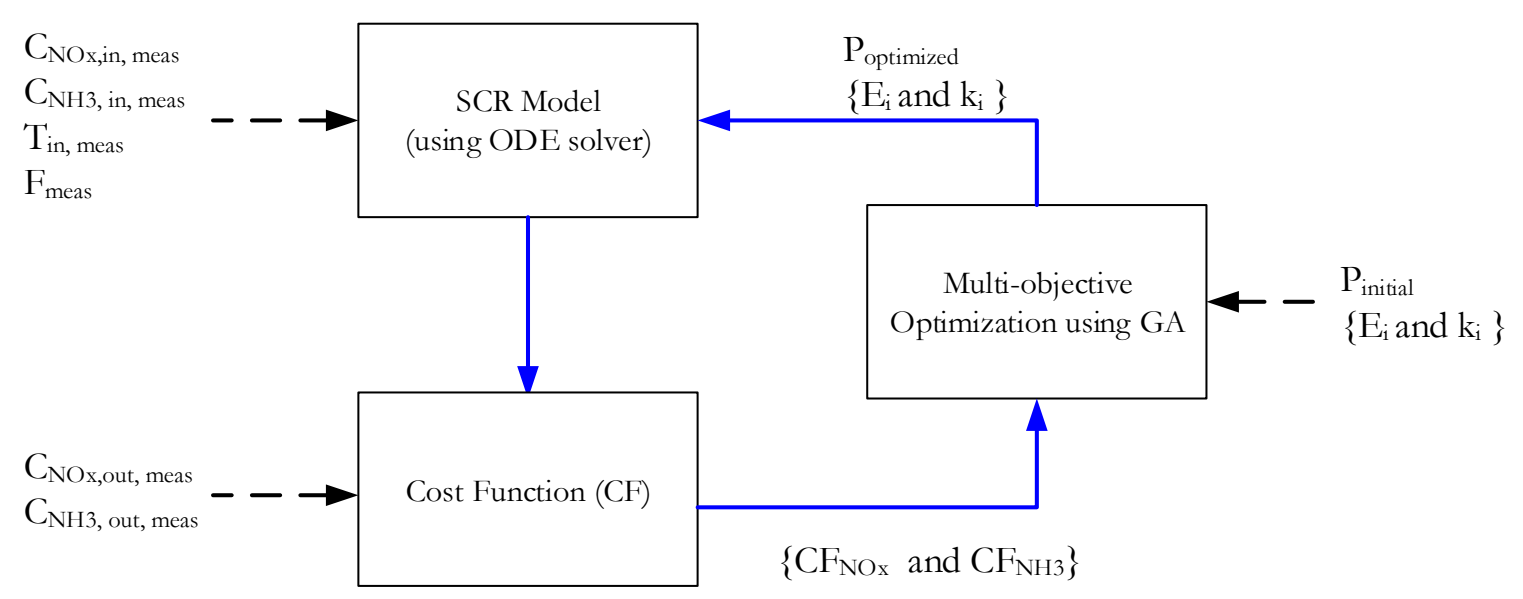

Figure 33. Schematic for model parameter identification 
The goal here is to minimize the error between the simulated value from the model and the measurement obtained from the experiment for a set of reaction parameters. The SCR model calibration was targeted to match the concentration of $\mathrm{NO}$ and $\mathrm{NH}_{3}$ to the SCR outlet values obtained in the steady-states experiments. The accuracy of the estimating the output variables evaluated by calculating the cost function, defined as sum of squared of accumulated error between the model prediction and the experimental measurement over the entire test. The equation for the considered cost function for each output variable is expressed as:

$$
\text { Cost Function }_{i}=\frac{1}{N} \sum_{t 0=1}^{N}\left(C_{i, \text { simulated }}-C_{i, \text { measured }}\right)^{2}
$$

where, $\mathrm{i}$ in the cost function is for the two species, $\mathrm{NO}$ and $\mathrm{NH}_{3} \cdot \mathrm{t}_{0}$ is the initial time and $\mathrm{N}$ is the total time of evaluation. For the GA optimization algorithm properties, the study uses the following default settings and are provided in the Table 5 below. Performance in changing optimization criterions were not examined for the study.

Table 5. GA Optimization Algorithm Criteria's

\begin{tabular}{|l|l|}
\hline \multicolumn{1}{|c|}{ GA Criteria Name } & \multicolumn{1}{c|}{ Criteria Used } \\
\hline Population Size & 100 \\
\hline Initial Range & Given (E's and k's ranges obtained from literature) \\
\hline Parameter Bounds & Given (E's and k's ranges obtained from literature) \\
\hline Parent Selection Criteria & Tournament (Size $=2$ ) \\
\hline Reproduction & Crossover fraction with 0.8 \\
\hline Mutation & Constraint dependent (Gaussian distribution) \\
\hline Crossover & Two Point \\
\hline Migration & Forward with last two individuals with 20 intervals \\
\hline Multi-objective Algorithm Setting & Distance measure with 0.35 pareto front population fraction \\
\hline Stopping Criteria & $\begin{array}{l}\text { Time Limit }=36000 \text { seconds } \\
\text { Stall Generation }=50 \\
\text { Function Tolerance }=0.0001\end{array}$ \\
\hline
\end{tabular}




\section{CHAPTER 5 Results and Discussion}

As the global objective of this study was to develop an ammonia reduction aftertreatment system for a heavy-duty natural gas engine which are subjected to stoichiometric combustion operation and consequently shown to produce high precursor ammonia tailpipe emission; the study addresses by conducting experimental evaluation on a 2008 8.9L natural gas engine which are known to have heavy market penetration in the vocational heavy-duty transport sector. The experiments and data analysis were performed in a sequential step and findings are summarized as per the studies approach towards the specific objectives.

\subsection{Baseline engine and after-treatment evaluation}

Baseline evaluation study was conducted to obtain the performance and emission levels of the procured 8.9L engine equipped with its stocked TWC system which were in-use application. Both the engine and TWC together already had approximately 13,800 hours of operating usage before the engine was installed on the engine dynamometer. The engine was tested under both transient and steady-state operating conditions in observe the dynamic characteristics of engine performance and composition of emission characteristics under such engine behavior and theirs results are provided in the following sections.

\subsubsection{Transient evaluation}

Table 6 summarizes the transient test matrix for ten experimental tests to evaluate the baseline setup. During the duration of the baseline testing, one of the FTIR system encountered sampling issues, and consequently the test was conducted with only one FTIR instrument sampling before or after the TWC system, as depicted by the test matrix in Table 6. The study conducted additional transient tests in order to obtain adequate information of the baseline engine and TWC setup evaluation. 
Table 6. Transient Test Matrix

\begin{tabular}{|c|c|c|c|}
\hline Test Cycles & FTIR [pre-TWC] & FTIR [post-TWC] & CVS System \\
\hline FTP 1 & Measured & Measured & Measured \\
\hline FTP 2 & Measured & NA & Measured \\
\hline FTP 3 & Measured & NA & Measured \\
\hline FTP 4 & Measured & NA & Measured \\
\hline FTP 5 & NA & Measured & Measured \\
\hline FTP 6 & NA & Measured & Measured \\
\hline FTP 7 & NA & Measured & Measured \\
\hline WHTC 1 & Measured & NA & Measured \\
\hline WHTC 2 & NA & Measured & Measured \\
\hline WHTC 3 & NA & Measured \\
\hline
\end{tabular}

Table 7 provides the summary results obtained from the carbon balanced iterative method with gaseous samples measured using the CVS system for the ten transient test cycles. It is to be noted that the engine was not preconditioned, and hence, each test cycle would differ in initial engine and after-treatment temperature state which could potentially affect the TWC temperature characteristics during the duration of the cycle. Additionally, some of the test cycles were not tested back-to-back, and the study considered using the initial TWC out temperature to define the test start type (i.e. cold start, warm start and hot start). In terms of total cycle work and brake specific CO2 values for the two-separate type of transient cycles (FTP and WHTC), the tests were repeatable with small variation, and potentially be due to test start type. Results indicated distinct observed in brake specific NOx emissions between cold-start, warm-start and hot-start test. Compared to cold-start test from the FTP results, the NOx emissions were on average of $38 \%$ and $71 \%$ lower than the warm-start and hot-start test, respectively. Similarly, from the WHTC results, the warm-start test showed on average 30\% lower brake specific NOx emission than the cold-start test. Results showed that the temperature activity of the aftertreatment TWC system does plays an important role in defining emission characteristics, especially for NOx. 
Table 7. Summary results from transient test cycles with baseline setup, CVS measured brake specific emission values

\begin{tabular}{|c|c|c|c|c|c|c|}
\hline \multirow{2}{*}{$\begin{array}{c}\text { Test } \\
\text { Cycles }\end{array}$} & \multirow{2}{*}{ Start Type } & $\begin{array}{c}\text { Cycle } \\
\text { Work } \\
\text { [bhp-hr] }\end{array}$ & \multicolumn{3}{|c|}{ Brake Specific Emissions [g/bhp-hr] } \\
\cline { 5 - 7 } & & $\mathrm{CO}_{2}$ & $\mathrm{NOx}$ & $\mathrm{CO}$ & THC \\
\hline FTP 1 & Warm-Start & 19.3 & 442.2 & 0.32 & 2.08 & 1.77 \\
\hline FTP 2 & Hot-Start & 19.3 & 442.2 & 0.14 & 2.08 & 1.44 \\
\hline FTP 3 & Warm-Start & 19.2 & 425.8 & 0.35 & 3.68 & 2.75 \\
\hline FTP 4 & Hot-Start & 19.0 & 427.3 & 0.17 & 1.95 & 1.92 \\
\hline FTP 5 & Warm-Start & 19.5 & 429.1 & 0.35 & 2.46 & 2.82 \\
\hline FTP 6 & Cold-Start & 19.5 & 434.4 & 0.52 & 3.35 & 2.61 \\
\hline FTP 7 & Cold-Start & 19.5 & 432.5 & 0.59 & 3.10 & 2.69 \\
\hline \multicolumn{2}{|c|}{ FTP Average } & 19.3 & 433.4 & 0.35 & 2.67 & 2.29 \\
\hline \multicolumn{2}{|c|}{ FTP Std. Deviation } & 0.2 & 6.7 & 0.16 & 0.70 & 0.56 \\
\hline WHTC 1 & Warm-Start & 26.0 & 407.2 & 0.45 & 2.86 & 2.93 \\
\hline WHTC 2 & Cold-Start & 26.1 & 404.6 & 0.70 & 2.89 & 3.39 \\
\hline WHTC 3 & Warm-Start & 26.0 & 406.9 & 0.53 & 2.51 & 3.21 \\
\hline \multicolumn{2}{|l|}{ WHTC Average } & 26.0 & 406.2 & 0.56 & 2.75 & 3.17 \\
\hline WHTC Std. Deviation & 0.02 & 1.42 & 0.13 & 0.21 & 0.23 \\
\hline
\end{tabular}

The transient experiments were also instrumented to measure concentration pre-andpost the TWC system to better understand the conversion efficiency of some of the anticipated emission species. Figure 34 shows averaged $\mathrm{NOx}, \mathrm{NH}_{3}, \mathrm{CO}$ and $\mathrm{THC}$ concentration values calculated over the entire FTP cycles and the seven FTP test repeats. For abovementioned species, the bar chart represents average concentration before and after the TWC system. In summary, the TWC reduced approximately $88 \%, 81 \%$ and $43 \%$ of $\mathrm{NOx}, \mathrm{CO}$ and THC components on average, respectively. On the other, the TWC produced approximately $39 \mathrm{ppm}$ of $\mathrm{NH}_{3}$ on average over the test cycles with standard deviation of $3 \mathrm{ppm}$ within the FTP tests.

Figure 35 includes result from the WHTC test results, showing similar trends in TWC reduction. For these cycles, the TWC reduced $89 \%, 84 \%$ and $27 \%$ of $\mathrm{NOx}, \mathrm{CO}$ and THC components, respectively. The THC conversion is observed lower than the FTP cycle which could also be due to the temperature activity of the WHTC cycle which included more low load operations than compared to FTP cycle. The cycles average $\mathrm{NH}_{3}$ concentration was observed to be at $61 \mathrm{ppm}$ with standard deviation of $4 \mathrm{ppm}$ within the WHTC tests. 

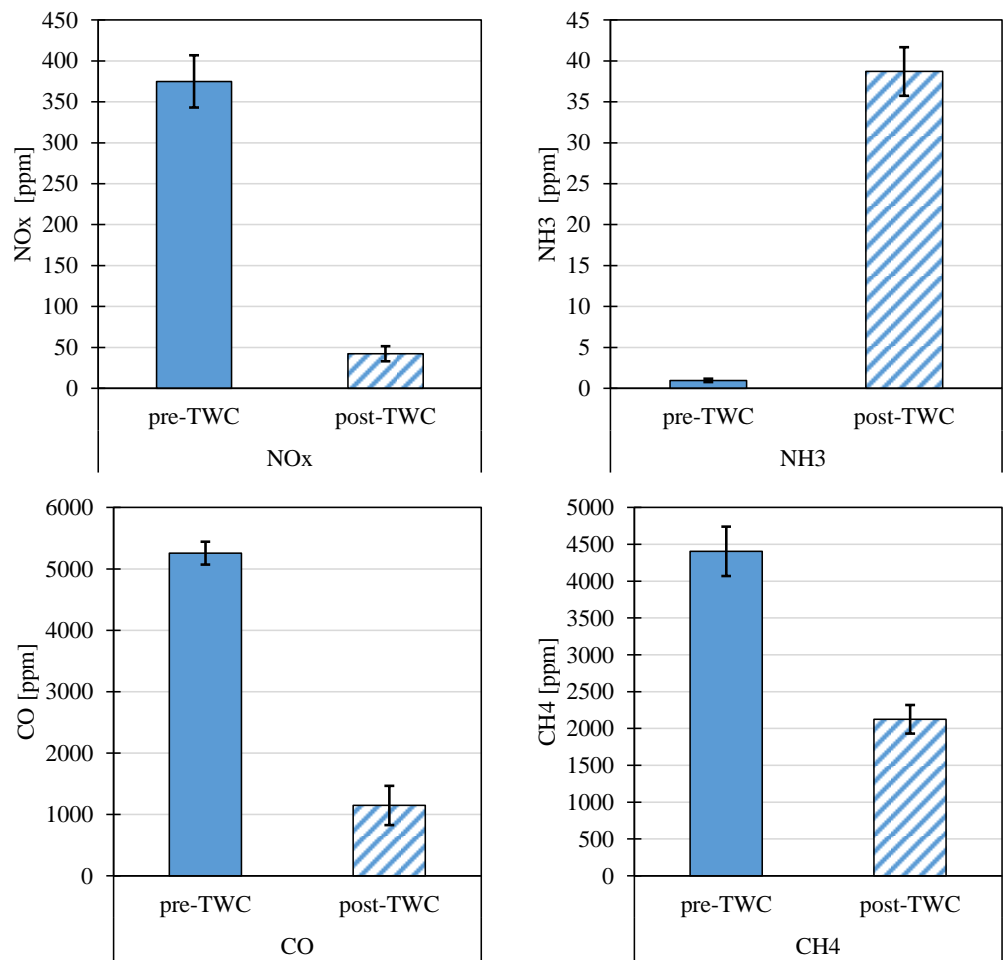

Figure 34. Averaged NOx, $\mathrm{NH}_{3}, \mathrm{CO}$ and THC emission concentration before and after the TWC system over transient (FTP cycle) tests
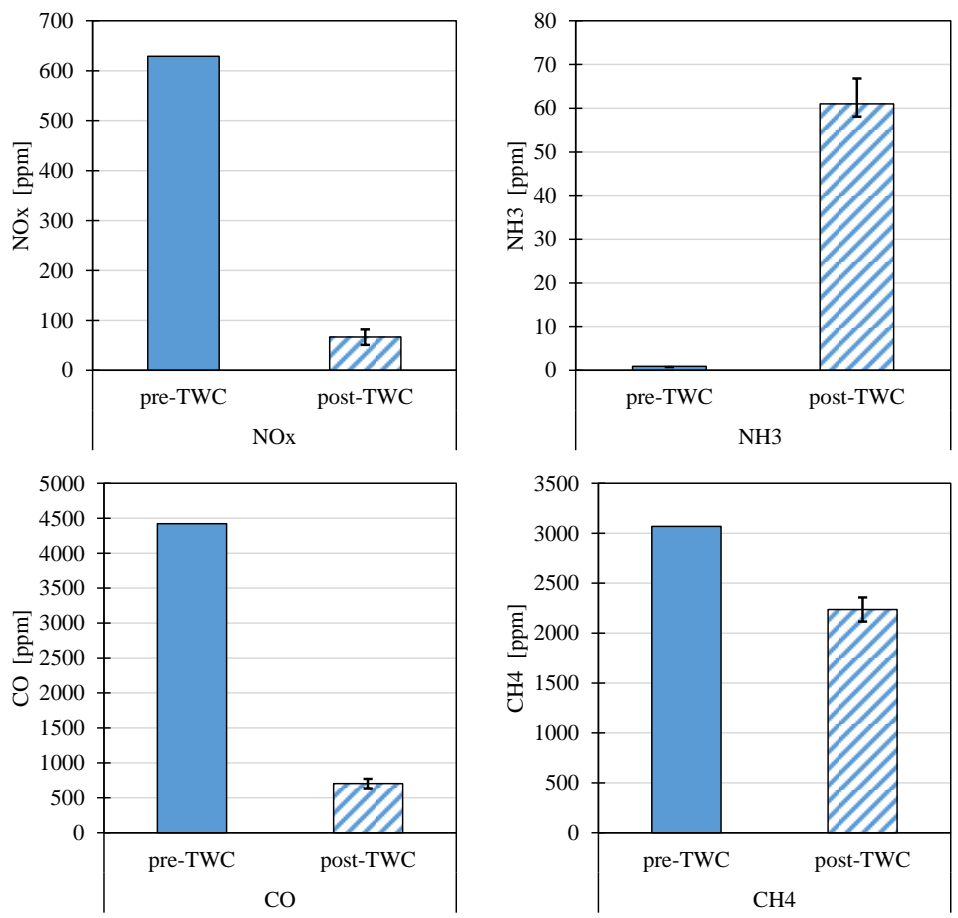

Figure 35. Averaged $\mathrm{NOx}, \mathrm{NH}_{3}, \mathrm{CO}$ and THC emission concentration before and after the TWC system over transient (WHTP cycle) tests 
The experimental study had only one FTP test (FTP-1) which measured concentration before and after the TWC using the two FTIR setup, and was hence used for the continuous evaluation of the selected species. Figure 36 shows the transient profile of the engine operation during the FTP cycle. The chart also includes the temperature profile (shown by the blue trace) averaged before and after the TWC. The maximum temperature was observed to be approximately $492^{\circ} \mathrm{C}$ for this specific cycle but did vary between different test repeats and cycle types. Results from the continuous NOx concentrations traces show that the sudden NOx peaks closely follow the transient engine behavior, and higher reductions of NOx are observed when the average temperature are at the higher thermal activity ranges, mainly above the lightoff temperatures of the catalyst. The light-off temperatures for any given species (defined as the temperature necessary to achieve $50 \%$ conversation efficiency, $T_{50}$ ) differ between catalyst formulation, and also between fresh versus aged catalyst (González-velasco et al. 2000). The traces of ammonia tend to correlate well with the transient behavior in the cycle, matching closely to change and aggressiveness of the engine power, agreeing well with relevant emission studies (Thiruvengadam et al. 2016). No inference can be made on the formation of precursor ammonia in the effect of changing temperature from the obtained charts but will be further analyzed based on grouping data into different temperatures ranges.
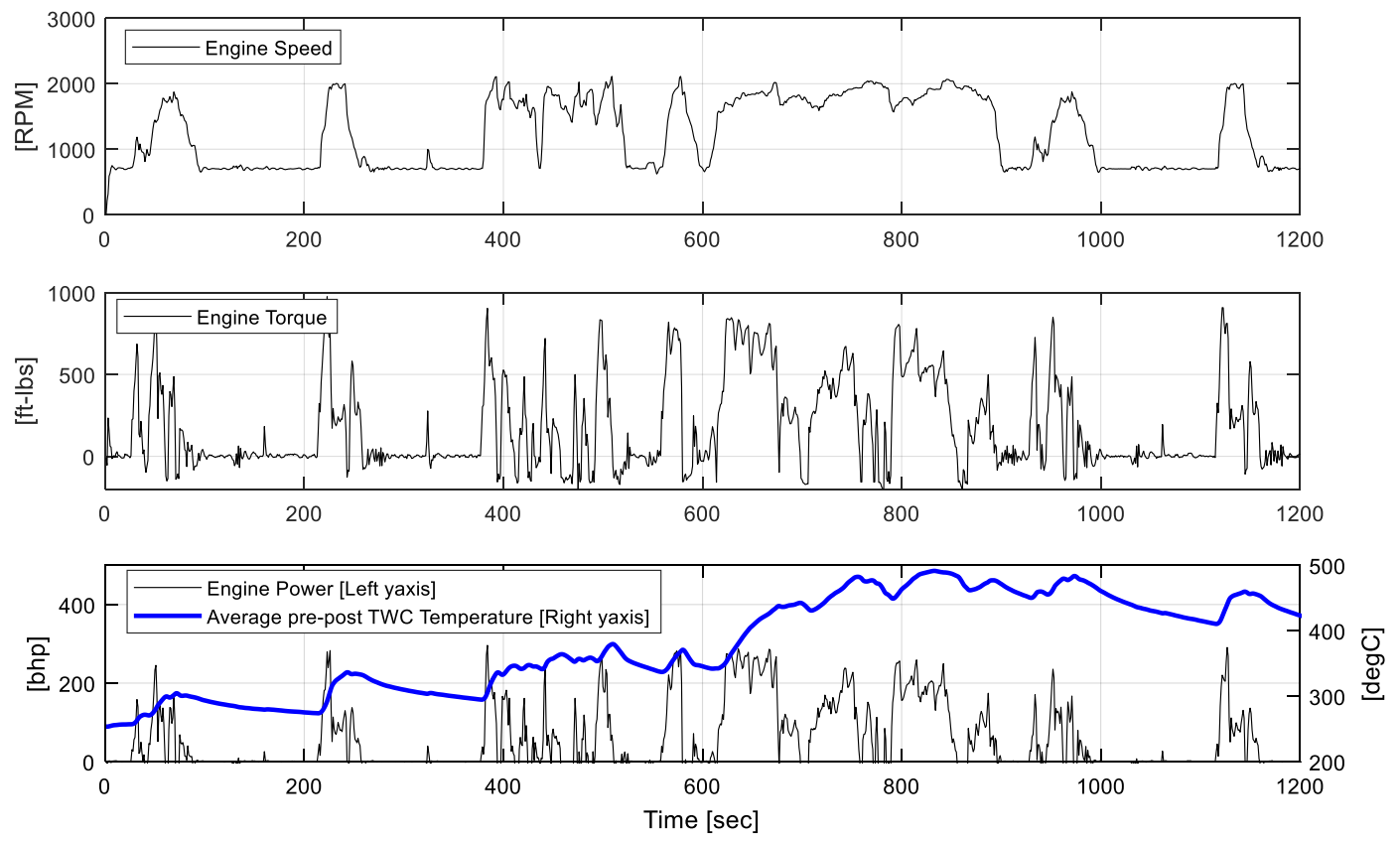

Figure 36. Engine transient operating (FTP-1 test) profiles showing engine speed (rpm), engine torque (ft-lbs), engine power (bhp) and averaged TWC temperature $\left({ }^{\circ} \mathrm{C}\right)$ 

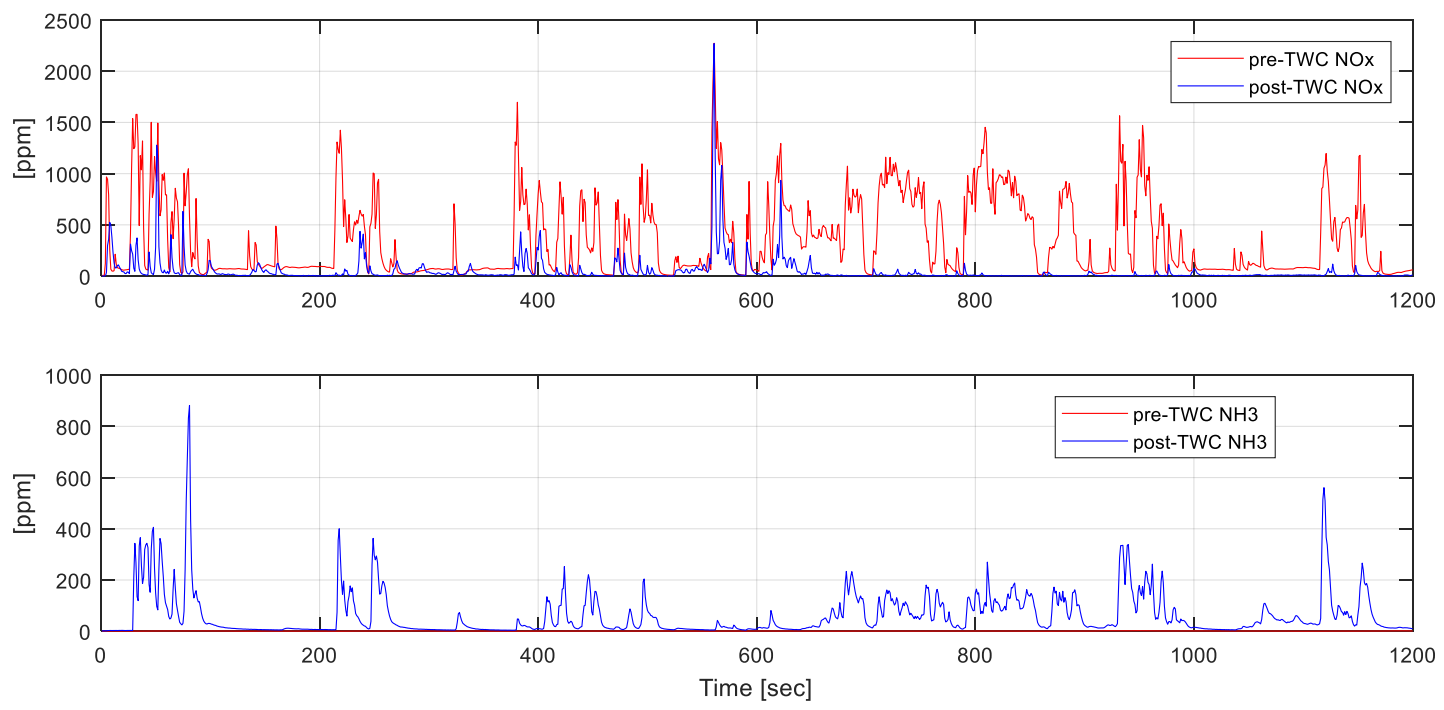

Figure 37. NOx and $\mathrm{NH}_{3}$ concentration before and after the TWC system over transient (FTP-2 test) operating condition

As the study looks into a passive-SCR system after the TWC as an ammonia reduction strategy, understanding the levels of available $\mathrm{NO}_{2} / \mathrm{NO}$ ratio before the SCR catalyst is also important. Figure 38 shows the continuous trace of $\mathrm{NO}_{2}$ and $\mathrm{N}_{2} \mathrm{O}$ concentration before and after the TWC system for the same FTP test (FTP-1). With the levels of $\mathrm{NO}_{2}$ observed at postTWC (average concentration of $1.5 \mathrm{ppm}$ ) and NO being the dominant species in total NOx, leading to a more standard-SCR reaction mechanism. Higher concentration of $\mathrm{N}_{2} \mathrm{O}$ traces were also observed after the TWC especially at lower temperatures during the first half of the cycle. Studies have shown the indication of $\mathrm{N}_{2} \mathrm{O}$ produced during operation of gasoline engine emission converters and the formation of $\mathrm{N}_{2} \mathrm{O}$ emission significantly correlates to the aggressive power demand in the cycle and are typically formed during redox reaction with $\mathrm{NO}$ by $\mathrm{CO}$ at lower temperatures $\left(200-400^{\circ} \mathrm{C}\right)$ (Mejía-Centeno and Fuentes 2009). 

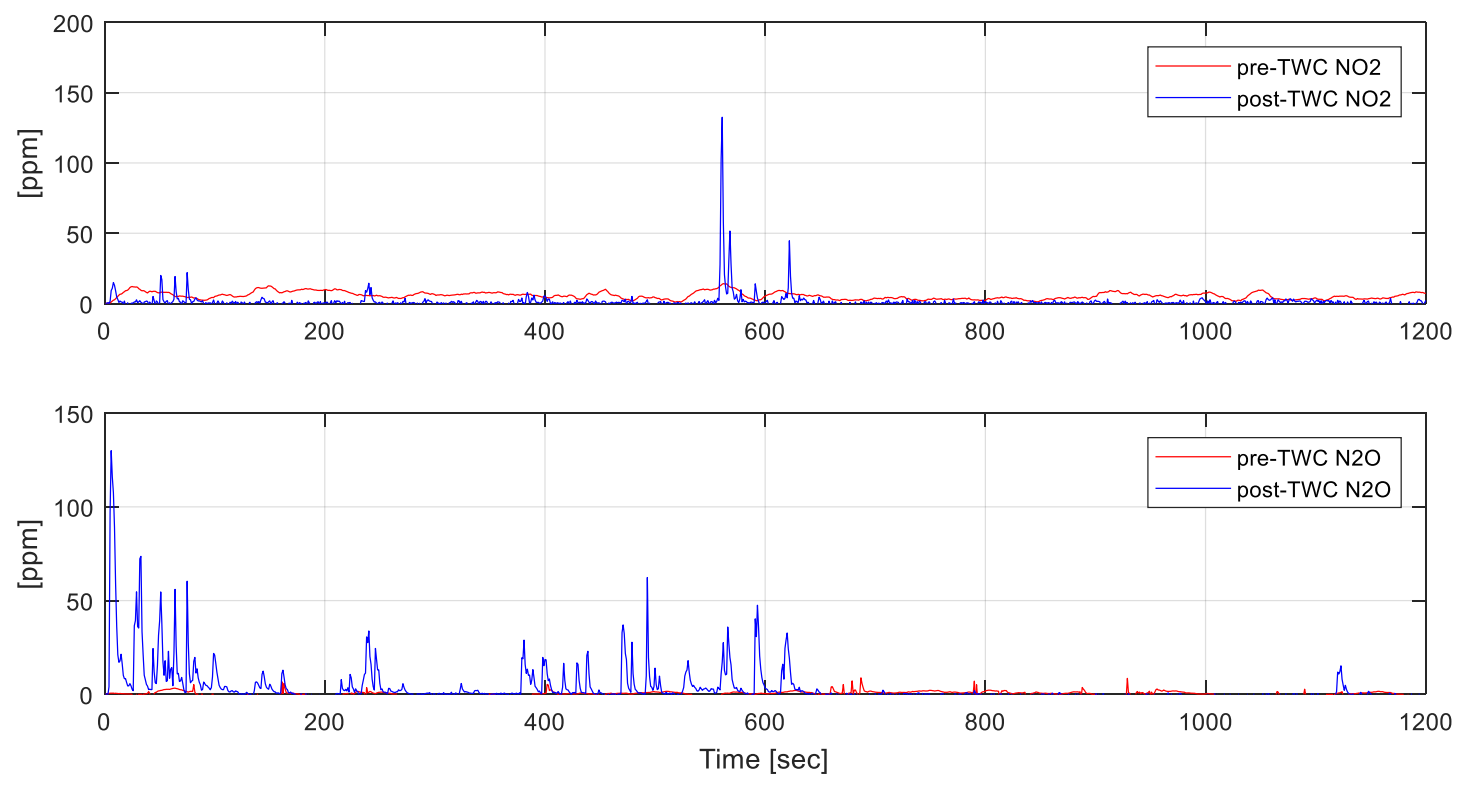

Figure 38. $\mathrm{NO}_{2}$ and $\mathrm{N}_{2} \mathrm{O}$ concentration before and after the TWC system over transient (FTP-1 test) operating condition

Figure 39 shows the average concentration of $\mathrm{NOx}$ and $\mathrm{NH}_{3}$ observed after the TWC evaluated from all the transient test cycles and grouped with respect to post-TWC temperature. The temperature consists of six bins with bin size of $50^{\circ} \mathrm{C}$. The result indicated higher NOx levels at lower temperature bins but with more variation in the values, and as observed from previous cases, a downward trend was observed with increasing temperature due to better conversion activity of the TWC at higher temperatures. On the other hand, we see a clear inclination in the rise of $\mathrm{NH}_{3}$ levels at higher temperature bins, indicating that the amount of $\mathrm{NH}_{3}$ formation in the TWC as a function of after-treatment temperature. The grey line with " $\mathrm{x}$ " markers represents the calculated $\mathrm{NH}_{3}$ to $\mathrm{NOx}$ ratio (ANR) using only the averaged $\mathrm{NOx}$ and $\mathrm{NH}_{3}$ values for each temperature bins. For three the temperature $\left(200-350^{\circ} \mathrm{C}\right)$ bin ranges, the ANR were calculated to be around 0.5 ratio with 1 (1:1 $\left.\mathrm{NH}_{3}: \mathrm{NOx}\right)$ being observed right around the $350-400^{\circ} \mathrm{C}$ temperature region, and then quickly rising with higher temperatures. 


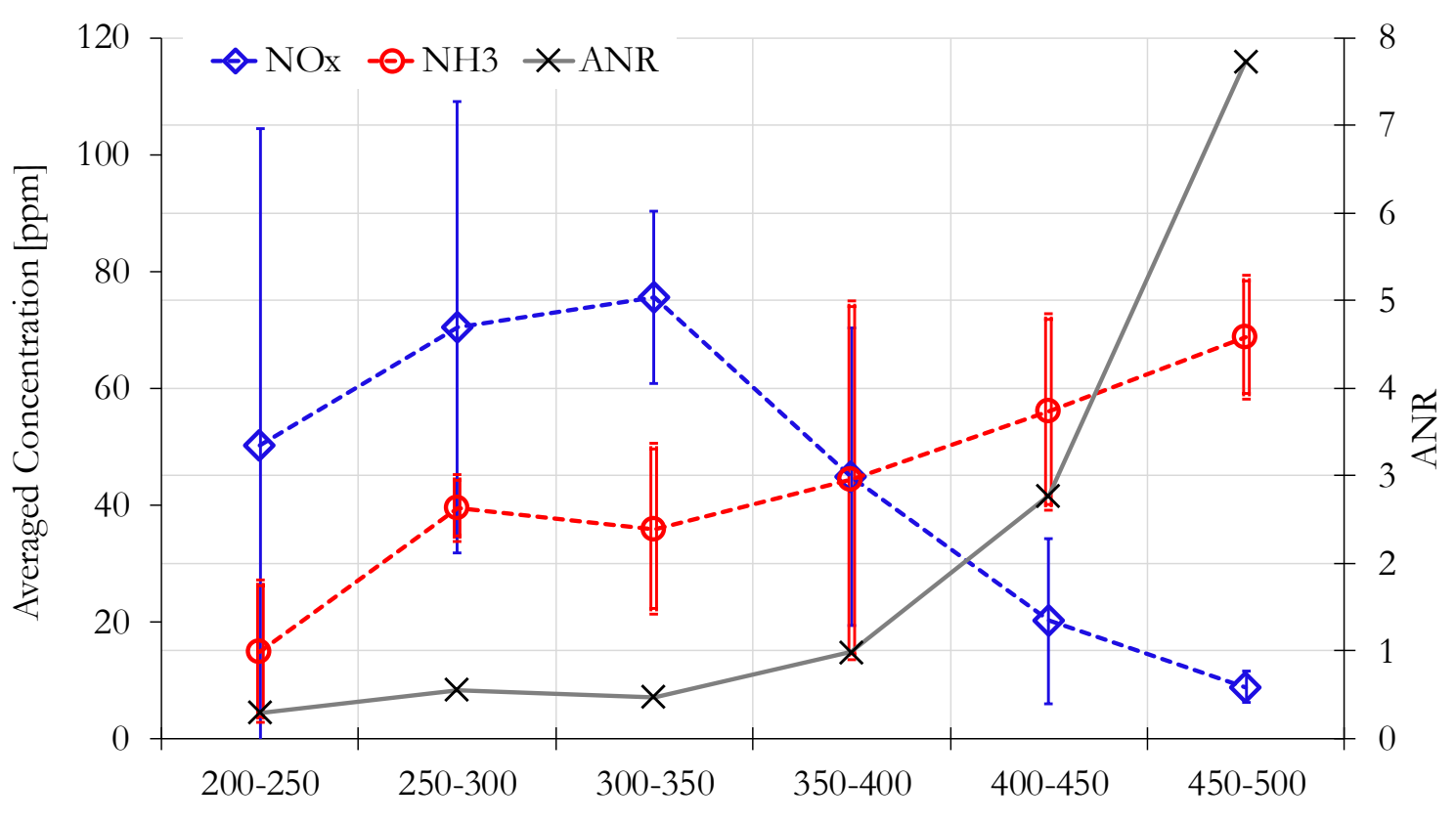

Temperature bins $\left[{ }^{\circ} \mathrm{C}\right]$

\section{Figure 39. Averaged post-TWC NOx and $\mathrm{NH}_{3}$ concentration for temperature bins from FTP and WLTC cycles}

\subsubsection{Steady-state evaluation}

The section here presents results obtained from the testing the engine under the 20 different steady-state modes. The results of $\mathrm{NOx}$ and $\mathrm{NH}_{3}$ concentration for each mode are averaged over the two-minute stabilization period of the test and the averaged concentration are plotted as a contour map under the engine speed and torque area, as shown by Figure 40. The results from the steady-state modes indicate NOx produced by the engine is distinctly higher in the upper region of low speed and high torque, and decreased at lower speed and torque modes. This engine parameters play a big role in combustion NOx formation which are closely related to combustion timings and fueling strategies, EGR rates which have shown to affect in-cylinder temperatures to promote $\mathrm{NO}_{\mathrm{x}}$ formation. The post-NOx results show mainly the TWC reduction characteristics, indicating adequate efficiency through the engine operation to quickly reach passed the light-off temperatures. On the other hand, the formation of ammonia is observed more in the region where higher NOx levels were observed, at low-speed high torque engine operations, directly relating to high temperature and richer air fuel ratio operating conditions. Further analysis showed that highest levels of $\mathrm{N}_{2} \mathrm{O}$ were observed at speed and torque operating modes during lower exhaust temperature TWC activity. 


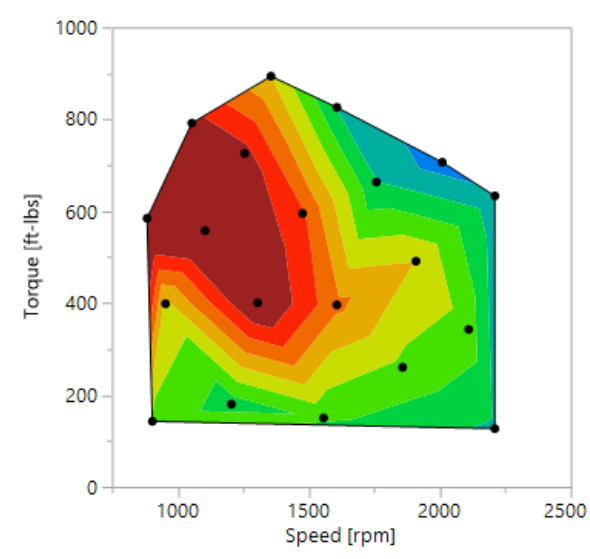

(a)

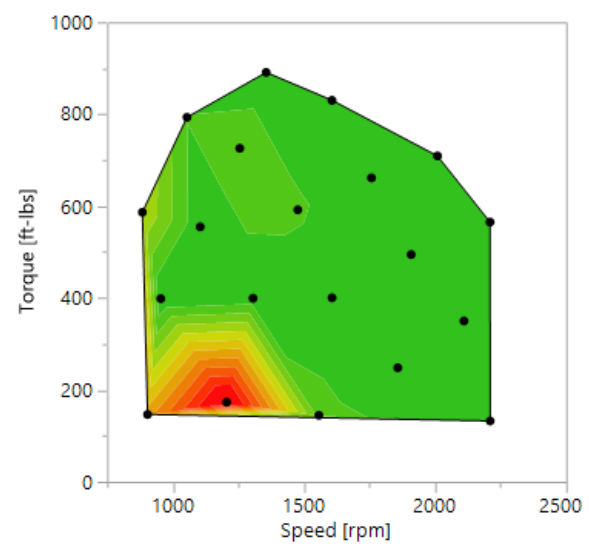

(c)
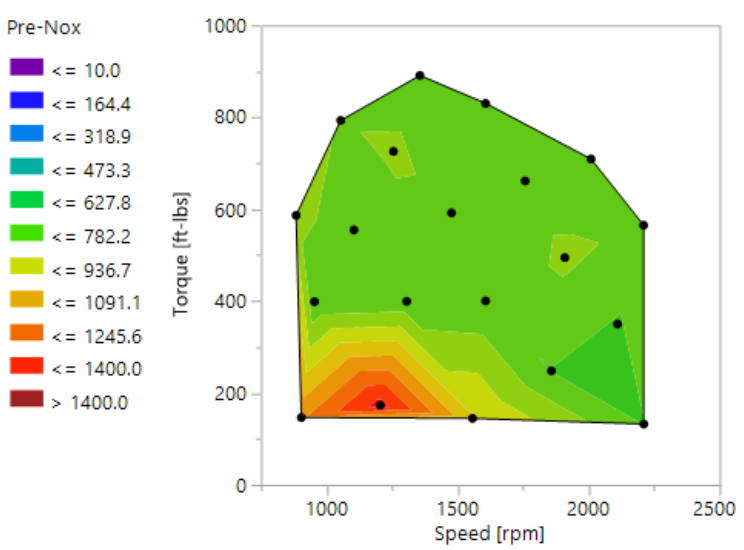

(b)
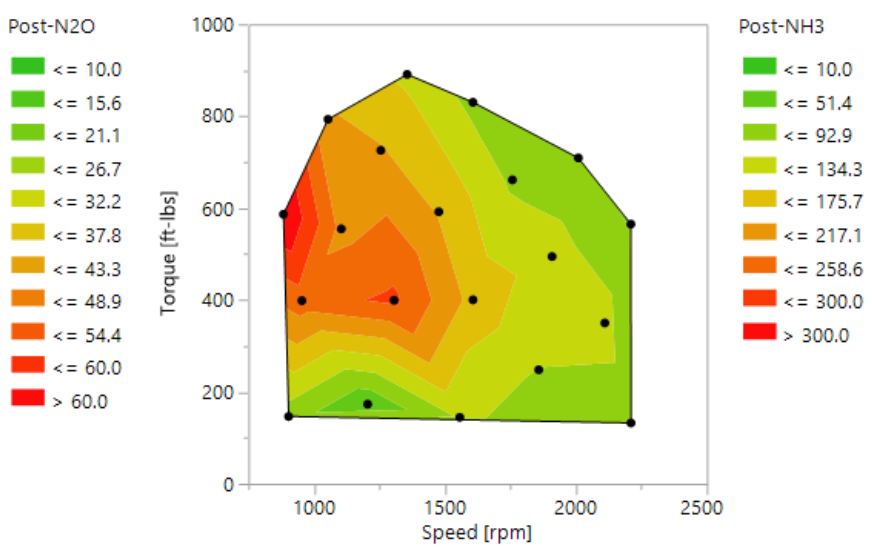

(d)

Figure 40. Concentration counter plots from 20 steady-state mode tests represented under engine speed and torque region; (a) pre-TWC NOx (b) post-TWC NOx, (c) post-N2O and (d) post-TWC $\mathrm{NH}_{3}$

\subsection{TWC+SCR after-treatment evaluation}

This section summarizes and discusses results obtained from conducting the five SCRs with three several types of catalyst formulation that were obtained from three separate catalyst manufactures. Details of the catalyst evaluation test matrix are provided by Table 8 . The study evaluated all five SCR catalyst over the FTP transient cycles with three repeats for each catalyst. Due to the size of the test matrix and availability of test time/budget, the SCRs were not tested over the WHTC cycle and the 20-mode steady-state study. Additional experiments and analysis were conducted for the SCR selected from the result obtained from the results obtained in this work. 
Table 8. Test matrix from the five SCR evaluation study

\begin{tabular}{|c|c|c|c|c|c|}
\hline $\begin{array}{c}\text { SCR } \\
\text { Number }\end{array}$ & SCR Type & Test Cycle & $\begin{array}{c}\text { FTIR } \\
\text { pre-TWC }\end{array}$ & $\begin{array}{c}\text { FTIR } \\
\text { post-TWC }\end{array}$ & $\begin{array}{c}\text { CVS } \\
\text { Measurement }\end{array}$ \\
\hline \multirow{3}{*}{ SCR 1} & \multirow{3}{*}{$\begin{array}{c}\text { Fe-Zeolite (Mfg: AP } \\
\text { Exhaust/ } 100 \text { cpsi) }\end{array}$} & FTP-1 & Measured & Measured & $\mathrm{NA}$ \\
\hline & & FTP-2 & Measured & Measured & Measured \\
\hline & & FTP-3 & Measured & Measured & Measured \\
\hline \multirow{3}{*}{ SCR 2} & \multirow{3}{*}{$\begin{array}{c}\text { Fe-Zeolite (Mfg: AP } \\
\text { Exhaust/ } 400 \text { cpsi) }\end{array}$} & FTP-4 & Measured & Measured & Measured \\
\hline & & FTP-5 & Measured & Measured & Measured \\
\hline & & FTP-6 & Measured & Measured & Measured \\
\hline \multirow{3}{*}{ SCR 3} & \multirow{3}{*}{$\begin{array}{l}\text { Fe-Zeolite (Mfg: } \\
\text { Corning/ } 400 \text { cpsi) }\end{array}$} & FTP-7 & Measured & Measured & Measured \\
\hline & & FTP-8 & Measured & Measured & Measured \\
\hline & & FTP-9 & Measured & Measured & Measured \\
\hline \multirow{3}{*}{ SCR 4} & \multirow{3}{*}{$\begin{array}{c}\text { Cu-Zeolite } \\
\text { (Cummins/400 cpsi) }\end{array}$} & FTP-10 & Measured & Measured & Measured \\
\hline & & FTP-11 & Measured & Measured & Measured \\
\hline & & FTP-12 & Measured & Measured & Measured \\
\hline \multirow{3}{*}{ SCR 5} & \multirow{3}{*}{$\begin{array}{c}V(M f g: \text { AP Exhaust/ } \\
100 \mathrm{cpsi})\end{array}$} & FTP-13 & Measured & Measured & Measured \\
\hline & & FTP-14 & Measured & Measured & Measured \\
\hline & & FTP-15 & Measured & Measured & Measured \\
\hline
\end{tabular}

The study evaluated the five SCR catalyst type based on the NOx conversion efficiency and $\mathrm{NH}_{3}$ storage characteristics over the transient FTP cycle. The SCRs activity in $\mathrm{NH}_{3}$ storage and $\mathrm{NOx}$ reduction in the presence the stored $\mathrm{NH}_{3}$ have shown to significantly depend upon the exhaust temperatures. It has been well observed from studies that the SCR substrate typically holds $\mathrm{NH}_{3}$ at lower temperatures and tend to gradually desorb $\mathrm{NH} 3$ as temperature of the SCR increases. Additionally, the efficient redox reactions are less likely to be observed at lower SCR temperatures due to catalyst inactivity below minimum activation or the light-off temperatures. And, this have shown to vary between SCR to SCR catalyst with different formulation and geometric characteristics (Colombo, Nova, and Tronconi 2010; Nagashima et al. 2000).

Figure 41 presents an example of the SCR 3 experimented over an FTP cycle, and the results are represented in terms of continuous $\mathrm{NOx}$ and $\mathrm{NH}_{3}$ concentration before and after the selected SCR. From initial visual inspection of the NOx profiles (referred to the top chart in the figure), its shows that NOx reduction tend to vary with temperature as commonly known property of SCR, especially Fe-zeolite based SCRs. We observed better NOx reduction at higher temperatures than the lower temperature seen in the first half of the FTP cycle. In addition to the temperatures, oxygen in exhaust also plays a significant role in promoting the standard-SCR reaction (Guralp et al. 2011). These can be observed from the high peak events during the instant power demands, especially during first half into the FTP cycle, where due to rich air-fuel operating conditions causes lack of lack of oxygen, and therefore inhibiting NOx reduction over 
the catalyst. In terms of $\mathrm{NH}_{3}$ slippage or storage characteristics (referred to the bottom chart in Figure 41), negligible slip in $\mathrm{NH}_{3}$ were observed after the SCR till 490 seconds into the FTP cycle, indicating complete $\mathrm{NH}_{3}$ adsorption. However, as temperature increased, low concentration of $\mathrm{NH}_{3}$ was observed, which indicates desorption, and the rate of $\mathrm{NH}_{3}$ slippage or desorption started increasing drastically with rise in temperature (around $400^{\circ} \mathrm{C}$ and above), around 650 seconds into the cycle. Further observing the profiles indicate that the $\mathrm{NH}_{3}$ releases by the SCR shows a slow cyclic response than compared to the inlet $\mathrm{NH}_{3}$ concentration profile, and these could be attributed to occurrence of ammonia desorption and part of inlet gaseous ammonia stored and slipped through the catalyst at different time periods. Additionally, the rate of adsorption and desorption are not uniform over the catalyst surface, which could vary with temperature dispersal within the SCR catalyst and differences in residence time due to change in exhaust flowrates.
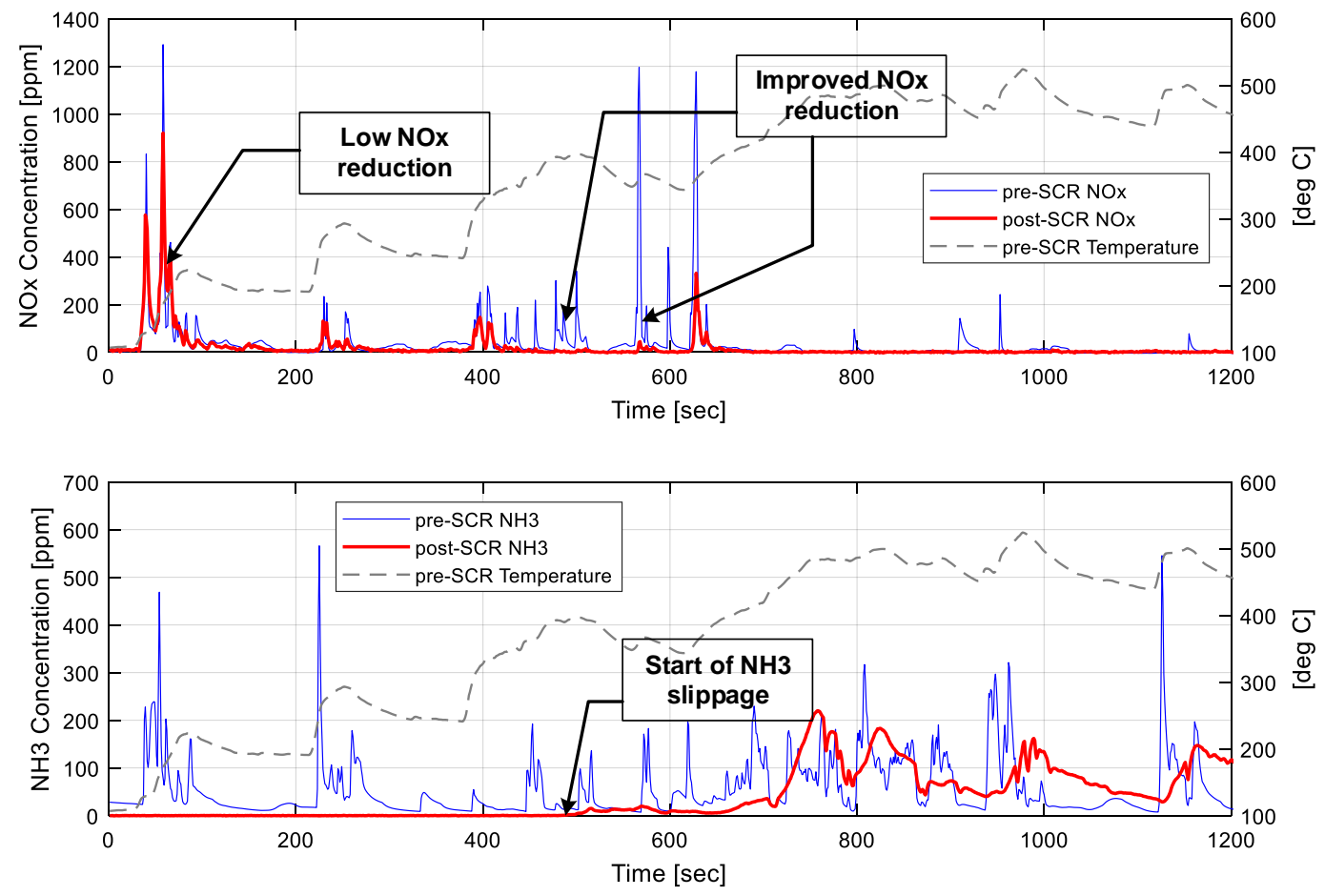

Figure 41. Profiles of NOx and $\mathrm{NH}_{3}$ concentration before and after the evaluated SCR over the FTP cycle (SCR 3)

The difference in the two curves (moles of $\mathrm{NH}_{3}$ entering and moles of $\mathrm{NH}_{3}$ leaving the SCR) provides a rough understanding of how much ammonia, in moles, are stored in the SCR catalyst at a given time and is provided by Figure 42 in moles of $\mathrm{NH}_{3}$ per catalyst volume 
$\left(\mathrm{mole} / \mathrm{m}^{3}\right)$. The analysis also shows that the ammonia storage value levels off at around 10-15 moles $/ \mathrm{m}^{3}$, indicating that the SCR can no longer absorb or store $\mathrm{NH}_{3}$ as a result of reaching the maximum ammonia storage capacity. The $\mathrm{NH}_{3}$ storage ratio at a given time cannot be directly measured and can only be estimated with prior knowledge the ammonia storage capacity. The ammonia storage ratio estimation also becomes challenging due to the uncertainty in how much ammonia had already been stored on the catalyst prior to the test and what percent of the stored ammonia has been used via different reactions. Additional the ammonia storage capacity of an SCR is not constant value and varies with temperature (Ciardelli et al. 2004).

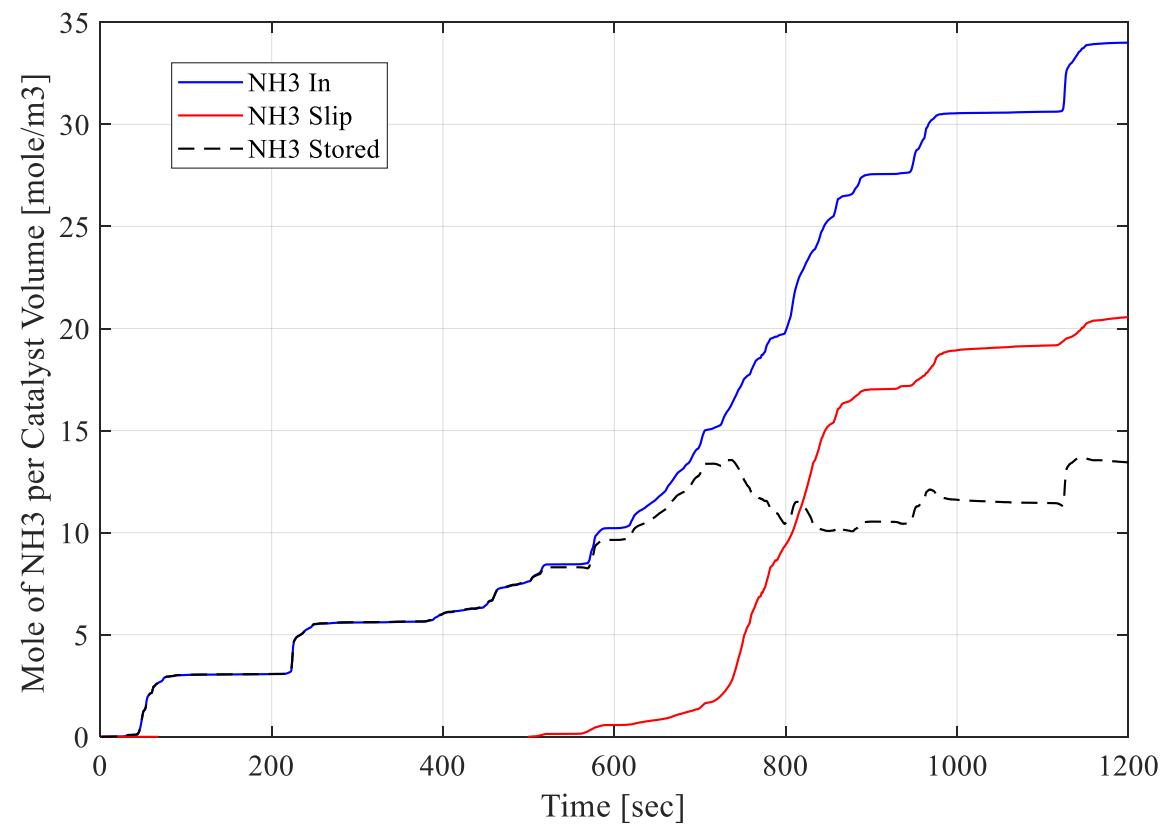

\section{Figure 42. Cumulative profile of inlet $\mathrm{NH}_{3}, \mathrm{NH}_{3}$ storage and $\mathrm{NH}_{3}$ slippage from the evaluated SCR 3 over the FTP cycle}

Based on the above-mentioned SCR characteristics, the study chooses to evaluate the five SCR for $\mathrm{NOx}$ conversion efficiency and $\mathrm{NH}_{3}$ storage over different temperature categories (bins) observed during the transient cycle from each test. The SCR inlet temperatures were categorized into six different ranges with a size range of $50^{\circ} \mathrm{C}$. The time-aligned concentration of $\mathrm{NOx}$ and $\mathrm{NH}_{3}$ before and after the SCR were subsequently averaged within the obtained temperature bins. Although, few tests did include data points below $200^{\circ} \mathrm{C}$ and above $500^{\circ} \mathrm{C}$, the chooses to evaluate the SCR performance only within the two given temperature ranges where majority of data were observed. 
Figure 43 provides the NOx conversion efficiencies obtained from the three repeated FTP tests and averaged for the six temperature ranges as represented by the horizontal axis. On comparing the five SCRs over different temperature ranges, SCR 1 (Fe-zeolite with 100cpsi) clearly indicates lowest conversion of NOx, resulting in efficiencies below $20 \%$ at both lower $(200-300)^{\circ} \mathrm{C}$ and higher $(350-500)^{\circ} \mathrm{C}$ temperature ranges. This could be attributed to the lower cell density formulation than compared to the other four SCRs, and studies have shown by increasing the cell density increases the SCR performance due to higher surface area (Chatterjee et al. 2005). This effect of higher cell density is indicative by the NOx conversion performance of SCR 2, an Fe-zeolite based SCR from the same manufacture with similar formulation but with cell density of 400 cpsi. However, both SCRs, 1 and 2 showed lower NOx conversion efficiency ay higher temperatures $(350-500)^{\circ} \mathrm{C}$ when compared to other SCRs, although, Fezeolite are known to perform well at these temperatures (Kröcher 2007). The observed performances from the two Fe-zeolite catalysts could be attributed to the wash-coat loading amount and the specific type of proprietary zeolite used to make the catalyst that was procured at the time of the study.

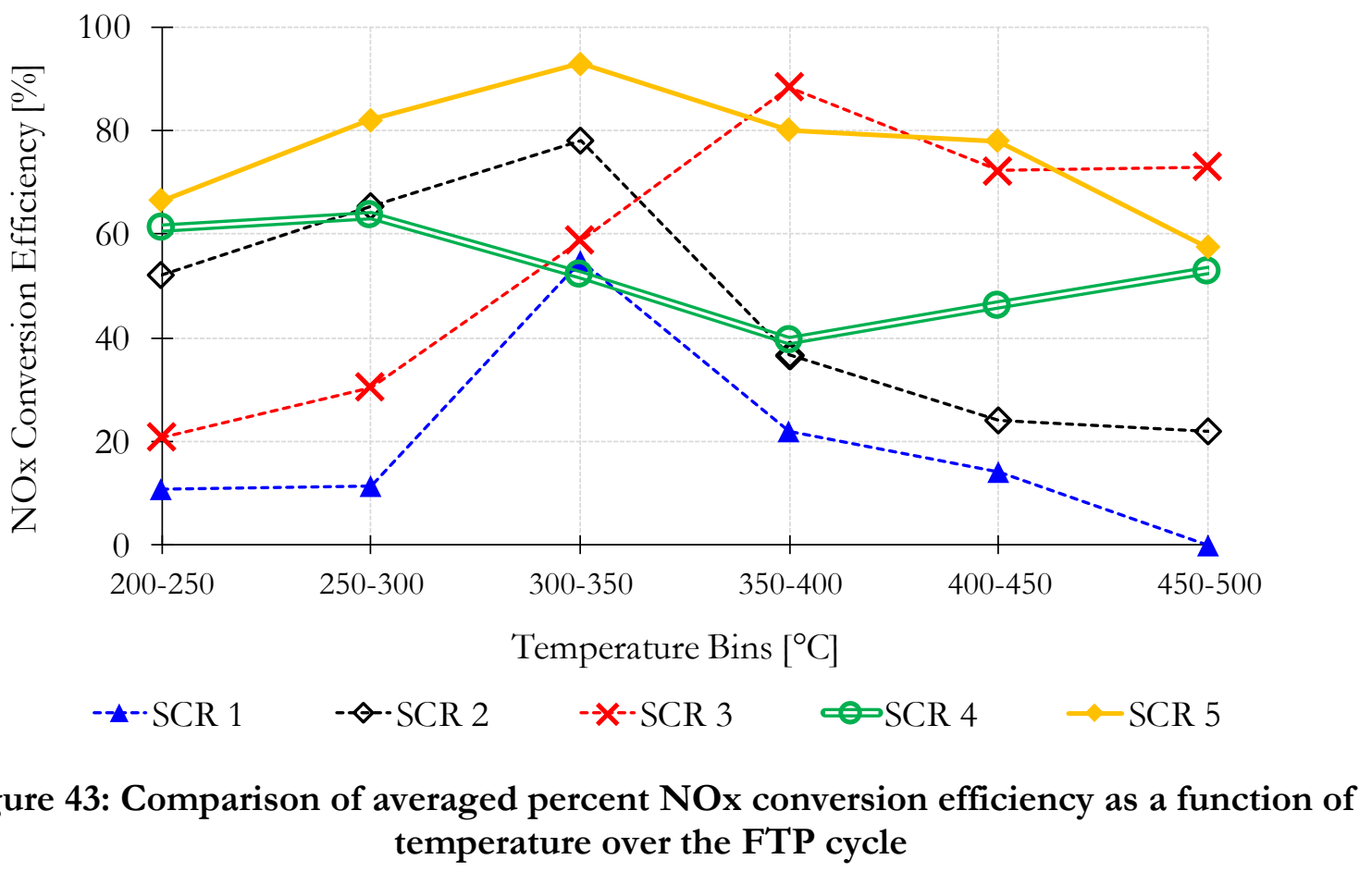

SCR 3, also a Fe-zeolite based catalyst (with 400 cpsi) but from a different manufacture, indicated improved conversion efficiency than compared to the other two Fe-zeolites based 
SCRs at higher temperature ranges. A peak of 88.5\% NOx conversion was observed at the 350$400^{\circ} \mathrm{C}$ temperature range but showed gradual decrease in conversion efficiency below these temperatures. The thermal activity of this SCR tends to agree with findings obtained in literatures.

The Cu-zeolite based (SCR 4 with 400 cpsi) catalyst, resulted in NOx conversion efficiencies within 40 to $60 \%$ over all evaluated temperatures ranges. However, most literatures have shown that $\mathrm{Cu}$-zeolite based SCRs tend to operate well than Fe- and V-based SCRs, especially at lower operating temperatures (Kröcher 2007). The lower observed Cu-zeolite performance could be significantly attributed to using a highly aged SCR catalyst obtained from a high mileage vehicle where durability studies have indicated deterioration of the SCR performance over time as a result of high thermal deactivation (Cavataio et al. 2008).

The last SCR evaluated, SCR 5 (V-based) catalyst, performed well on NOx conversion efficiency over all the temperature ranges when compared to majority of other four SCRs. It also showed the highest conversion efficiency at lower temperatures when compared against the two zeolite based SCRs. Several studies have indicated that V-based catalyst operates at best in temperature ranges between $(250-450)^{\circ} \mathrm{C}$ (Majewski 2005; Kröcher 2007), which tracks to the result obtained in this study.

In concluding the SCR evaluation on NOx conversion performance, it is to be noted that the study evaluated the SCR's performance using averaged pre-and-post SCR temperatures and concentrations over extremely dynamic behavior of the engine and after-treatment operating conditions. Additionally, due to studies experimental limitations, the SCR properties were not held at same input parameters, and therefore could have led to disparities in performance curves as observed in multiple literatures. Further investigation needs to be performed to elaborate the performance of different input variables under such temperature ranges and conditions observed in stoichiometric natural gas exhaust downstream of a TWC. 


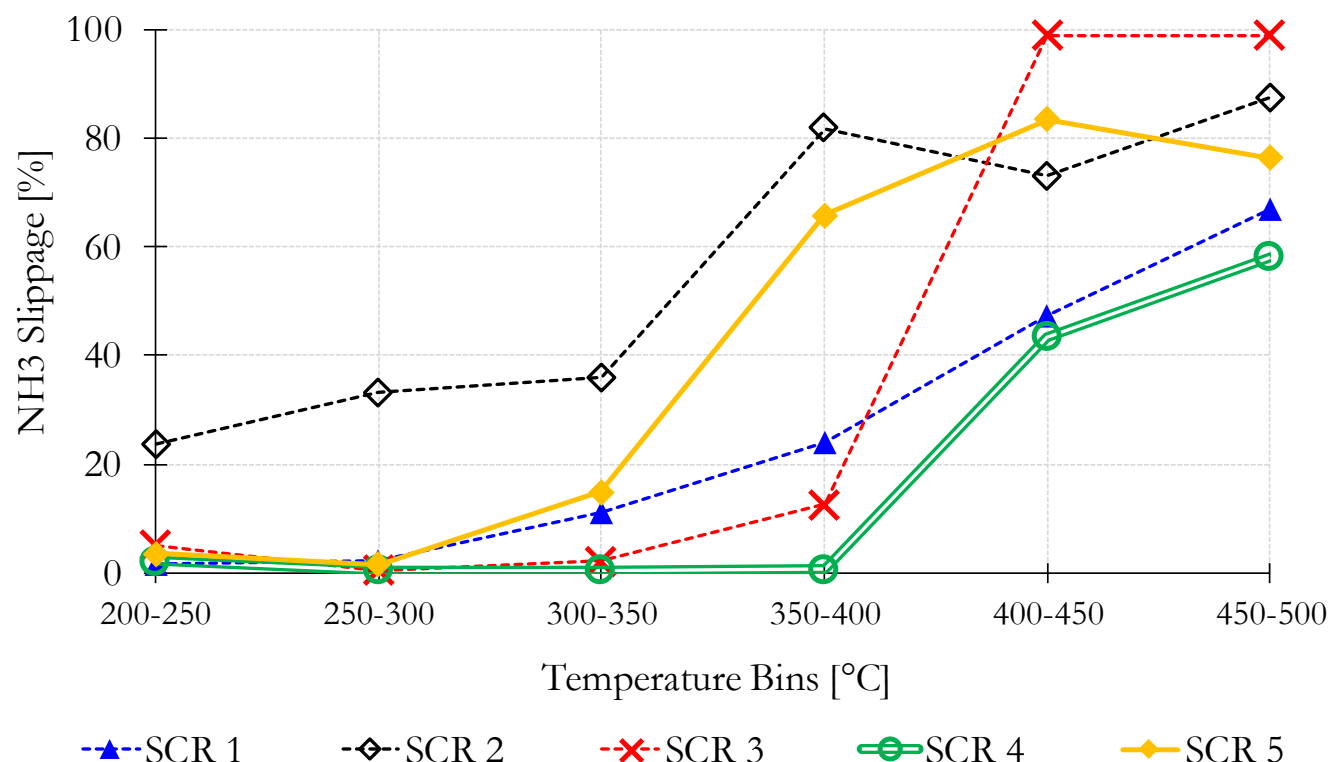

Figure 44: Comparison of averaged percent $\mathrm{NH}_{3}$ slip as a function of temperature over the FTP cycles

On the other hand, Figure 44 shows the averaged ammonia slippage in percent for all five SCR catalyst tested over the same six binned temperature ranges. Results indicate a similar trend from all five SCRs with $\mathrm{NH}_{3}$ being absorbed at lower temperature ranges, and as temperature increased the slippage of $\mathrm{NH}_{3}$ also showed to increase. Cu-zeolite based catalyst (SCR 4) indicated the least percent of $\mathrm{NH}_{3}$ slip from $(200-400)^{\circ} \mathrm{C}$ temperature ranges when compared to other four SCRs, and $\mathrm{NH}_{3}$ slippage was observed from $400{ }^{\circ} \mathrm{C}$ and above. SCR 3 which showed higher conversion efficiency for $\mathrm{NOx}$ at higher temperatures above $400^{\circ} \mathrm{C}$ resulted in slipping $100 \%$ of $\mathrm{NH}_{3}$ entering the catalyst. The Fe-zeolite based catalyst with 400 cpsi (SCR 2) resulted in the highest $\mathrm{NH}_{3}$ slippage, specifically at lower temperatures, even though it performed well on NOx conversion efficiency when compared to the other two Fezeolite based SCRs.

Figure 45 presents the overall percent result of the $\mathrm{NOx}$ conversion efficiency and $\mathrm{NH}_{3}$ slippage averaged over the entire cycle test. The chart basically provides the averaged performance of the added SCR systems to reduce NOx that wasn't reduced by the TWC, and capture the precursor $\mathrm{NH}_{3}$ emission that was formed during the TWC reactions. In summary, the V-based catalyst (SCR 5) resulted in maximum NOx conversion efficiency of $76.2 \%$ on average but with a higher $\mathrm{NH}_{3}$ slippage percent of $40.9 \%$. On the other hand, the Fe-zeolite with 100 cpsi (SCR 1) gave the lowest NOx reduction with average value of $18.8 \%$ but had a 
moderate slippage of $\mathrm{NH}_{3}(25.5 \%)$ when compared to other SCRs. SCR 4 (Cu-zeolite) catalyst with average NOx reduction of $52.7 \%$ and lowest $\mathrm{NH}_{3}$ slippage percent of $17.5 \%$ was considered for further adsorption/desorption study that was utilized for the development of the control regeneration strategy and SCR modeling. Another reason for the study to select this SCR was based on the overall higher temperature activity evident in stoichiometric natural gas engines, where ammonia storage capacity and NOx conversion efficiency becomes important factors in developing a passive-ARC system.

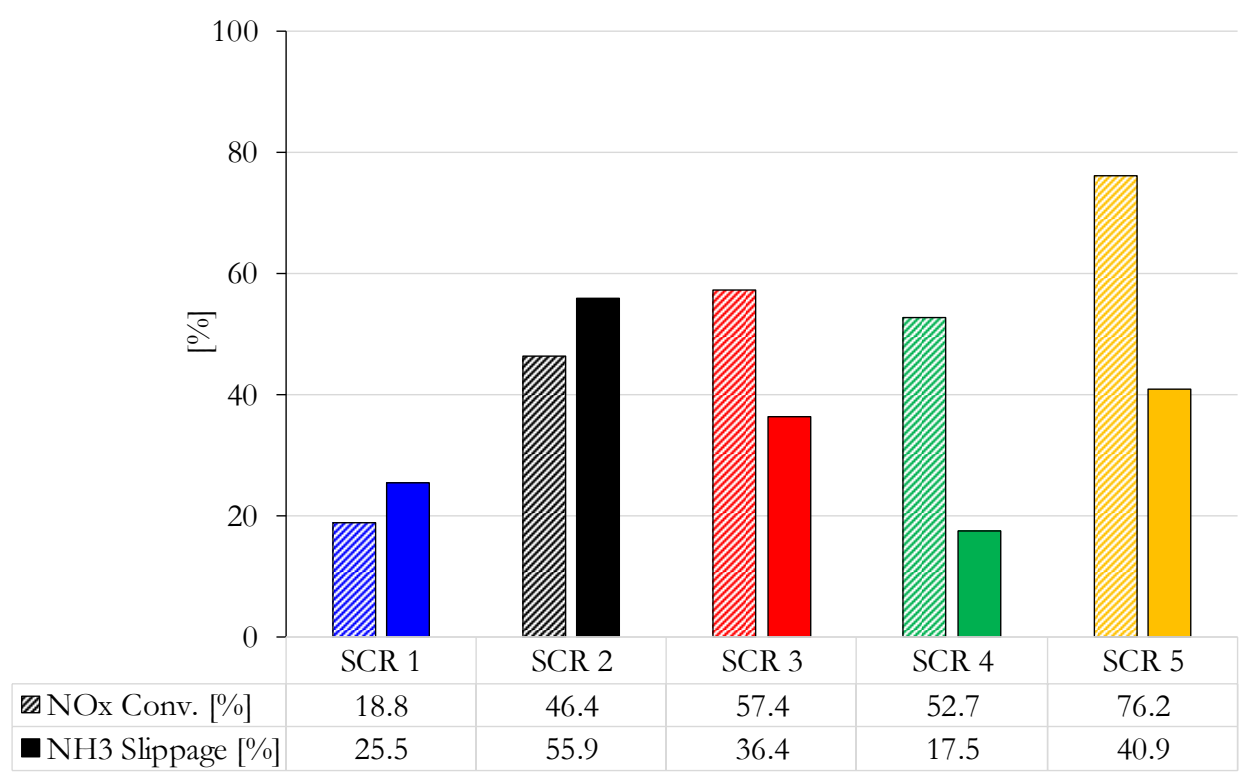

Figure 45. Average NOx conversion efficiency and $\mathrm{NH}_{3}$ slippage for all five SCR catalyst over the entire three repeated FTP cycles

\subsection{Ammonia adsorption/desorption evaluation (SCR 4)}

The $\mathrm{NH}_{3}$ storage ratio cannot be directly measured due to the process adsorption and desorption observed at different time scales over the catalyst surface. However, the storage behavior of the catalyst can be estimated based on prior knowledge catalyst ammonia storage capacity and storage ratio over the SCR surface. Therefore, any steady-state mode under a given engine operations and duration must be analyzed as time dependent rate. This section includes a comprehensive analysis of the eight steady-state mode tests results in order to evaluate the adsorption/desorption characteristics under stable engine operating conditions. Table 9 list the engine operating condition along pre-and-post SCR temperatures averaged over stable ready of each 8 -steady state modes. The duration of each mode varied in total duration based on visual 
inspection of the FTIR concentration traces and different stabilization time under different engine operating conditions. Each mode also includes manual control of lean-ON periods in order to induce variation in $\mathrm{NH}_{3}$ and $\mathrm{NOx}$ dynamics and recognize rate of stabilize time on various species based on lean-ON durations. The results and analysis from this study will be used in control and model development as presented in the follow sections.

Table 9. Steady-State mode test for SCR 4, engine and SCR temperature operating conditions

\begin{tabular}{|c|c|c|c|c|c|}
\hline Mode & $\begin{array}{c}\text { Engine Speed } \\
{[\mathrm{rpm}]}\end{array}$ & $\begin{array}{c}\text { Engine Torque } \\
{[\mathrm{ft}-\mathrm{lbs}]}\end{array}$ & $\begin{array}{c}\text { Engine } \\
\text { Power } \\
{[\mathrm{bhp}]}\end{array}$ & $\begin{array}{c}\text { Average pre-SCR } \\
\text { Temperature }\left[{ }^{\circ} \mathrm{C}\right]\end{array}$ & $\begin{array}{c}\text { Average post- } \\
\text { SCR Temperature } \\
{\left[{ }^{\circ} \mathrm{C}\right]}\end{array}$ \\
\hline 1 & 751 & 247 & 35.4 & 320.0 & 190.3 \\
\hline 2 & 851 & 84 & 13.6 & 330.8 & 218.0 \\
\hline 3 & 1249 & 316 & 75.1 & 508.8 & 403.4 \\
\hline 4 & 1296 & 37 & 9.2 & 319.9 & 222.6 \\
\hline 5 & 1480 & 597 & 168.3 & 572.6 & 473.4 \\
\hline 6 & 1521 & 162 & 46.8 & 468.2 & 275.9 \\
\hline 7 & 1575 & 337 & 101.1 & 554.2 & 433.3 \\
\hline 8 & 1611 & 683 & 209.4 & 572.7 & 486.4 \\
\hline
\end{tabular}

Figure 46 displays results from mode 1, a low speed and load engine condition operated for 1000 second duration resulting in an average pre-SCR temperature of approximately $320^{\circ} \mathrm{C}$. For the entire duration of the mode, the catalyst absorbed ammonia without any noticeable ammonia slippage after the SCR. Reduction of NOx is well observed in the beginning of the mode (20 to 290 secs), with NOx conversion decreasing as SCR inlet NOx stabilizes around $550 \mathrm{ppm}$. And, this could potentially be due to low availability of $\mathrm{NH}_{3}$ stored in the catalyst, as $\mathrm{NH}_{3}$ entering the catalyst is also low. From the stoichiometric form of $\mathrm{NOx}$ reduction, shows that 1 mole of $\mathrm{NH}_{3}$ is needed to react with 1 mole of $\mathrm{NOx}$. Hence, the number of mole of ammonia used for reduction of NOx is equal to the number of moles of NOx. In a steady-state condition where the exhaust flow rate is constant, it can be said that the different between NOx concentration before and after the SCR should account for the number of $\mathrm{NH}_{3}$ value used in the catalyst, assuming no reaction other than the reduction ( $\mathrm{NOx}$ and $\mathrm{NH}_{3}$ ) occurs. However, at the end of the steady-state value of NOx, at around 220 seconds, we notice traces of $\mathrm{NO}_{2}$ at the SCR outlet and this can be interpreted from possible oxidation reactions between $\mathrm{NH}_{3}$ and $\mathrm{O}_{2}$ or $\mathrm{NO}$ and $\mathrm{O}_{2}$ (Grossale, Nova, and Tronconi 2008; M. F. Hsieh and Wang 2011). It is to be noted that, $\mathrm{NO}_{2}$ values before the SCR was observed to be close to zero, and would have 
negligible effect in NOx reduction in the catalyst, hence, were not provided in the charts below. At 300 seconds into the mode, the lean-ON mode was manually turned on to operate the engine into a more leaner operation instead of stoichiometric baseline operation. The start of the leanON period can be noticed from the rise of NOx before the SCR due to reduced efficiency of TWC in converting NOx during high oxygen exhaust events. The pre-SCR NOx rises and stabilizes around roughly $860 \mathrm{ppm}$. We observe a noticeable difference in post-SCR NOx rate in the beginning of the lean-ON period due to NOx conversion reactions and slowly approached the SCR inlet NOx levels. In terms of NOx split at the SCR outlet, NO remained approximately stable but gradual rise in $\mathrm{NO}_{2}$ was observed. Concentration of $\mathrm{NO}_{2}$ increased and stabilized close to the NO concertation value. Higher oxygen content due to lean operation of the engine could have contributed it favor more of an oxidation reaction between $\mathrm{NO}$ and $\mathrm{O}_{2}$ than compared to the reaction between $\mathrm{NH}_{3}$ and $\mathrm{O}_{2}$. The oxidation of the absorbed $\mathrm{NH}_{3}$ reactions are observed typically at temperatures higher than $450^{\circ} \mathrm{C}$ (M. F. Hsieh and Junmin Wang 2010), and the modes average pre-and-post SCR temperature was at $255^{\circ} \mathrm{C}$. At about 596 seconds, the lean-ON period was turned off, and the experiment was operated back to baseline stoichiometric engine condition. However, only minimum to no difference in total NOx value were observed during this period of the mode 1, but with an average approximate split of $30 \%$ $\mathrm{NO}_{2}$ and $70 \% \mathrm{NO}$. In summary, the oxidation reaction was seen to be more dominant than the reduction reaction due to low $\mathrm{NH}_{3}$ availability in the catalyst. 

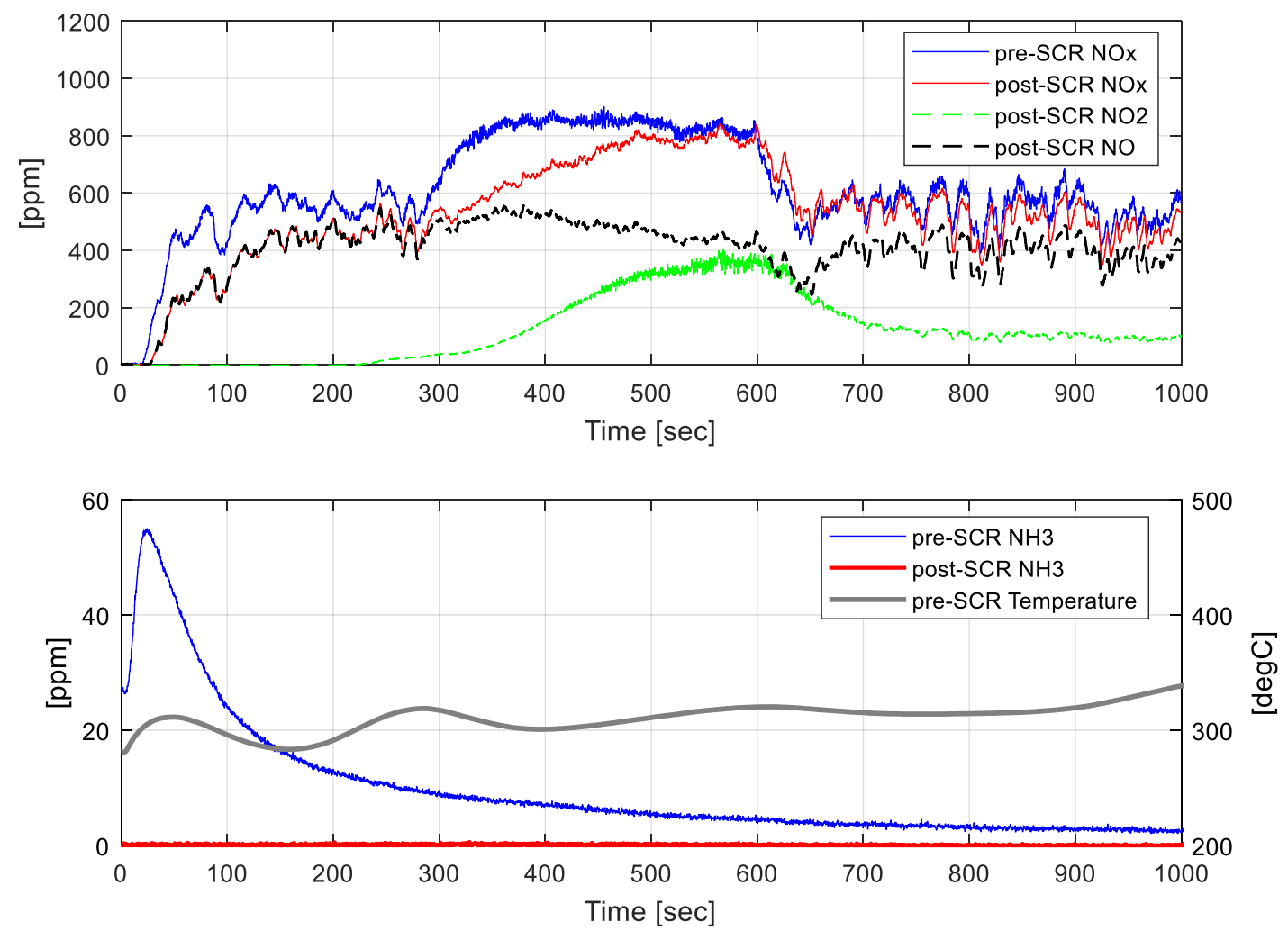

Figure 46: Mode 1 [engine speed $=751 \mathrm{rpm}$, engine torque $=247 \mathrm{ft}-1 \mathrm{bs}$ ]; comparing pre-and-post $\mathrm{NOx}$ and $\mathrm{NH}_{3}$ concentrations; includes lean-ON period (300-596 seconds)

Another similar engine operating condition, steady-state Mode 2 results are provided in Figure 47 having on average of $10^{\circ} \mathrm{C}$ higher SCR inlet temperature than the previous mode (Mode 1). Similar to the previous modes NOx observation, Mode 2 also had higher NOx conversion in the beginning of the mode and showing a decreasing trend as inlet NOx stabilized around 400 seconds into the mode. The $\mathrm{NH}_{3}$ entering the SCR was observed to be significantly low (4 ppm on average for entire 1000 second mode duration), and all $\mathrm{NH}_{3}$ produced by the TWC was stored in the SCR catalyst. The lean-ON period was switched on at 480 seconds and turned off at 700 seconds into the mode. Majority of the reaction could be contributed to $\mathrm{NH}_{3}$ and $\mathrm{NO}$ reduction reaction in presence of excess oxygen. Similar trend in rise of $\mathrm{NO}_{2}$ was observed doe to occurrence of oxidation reaction. 

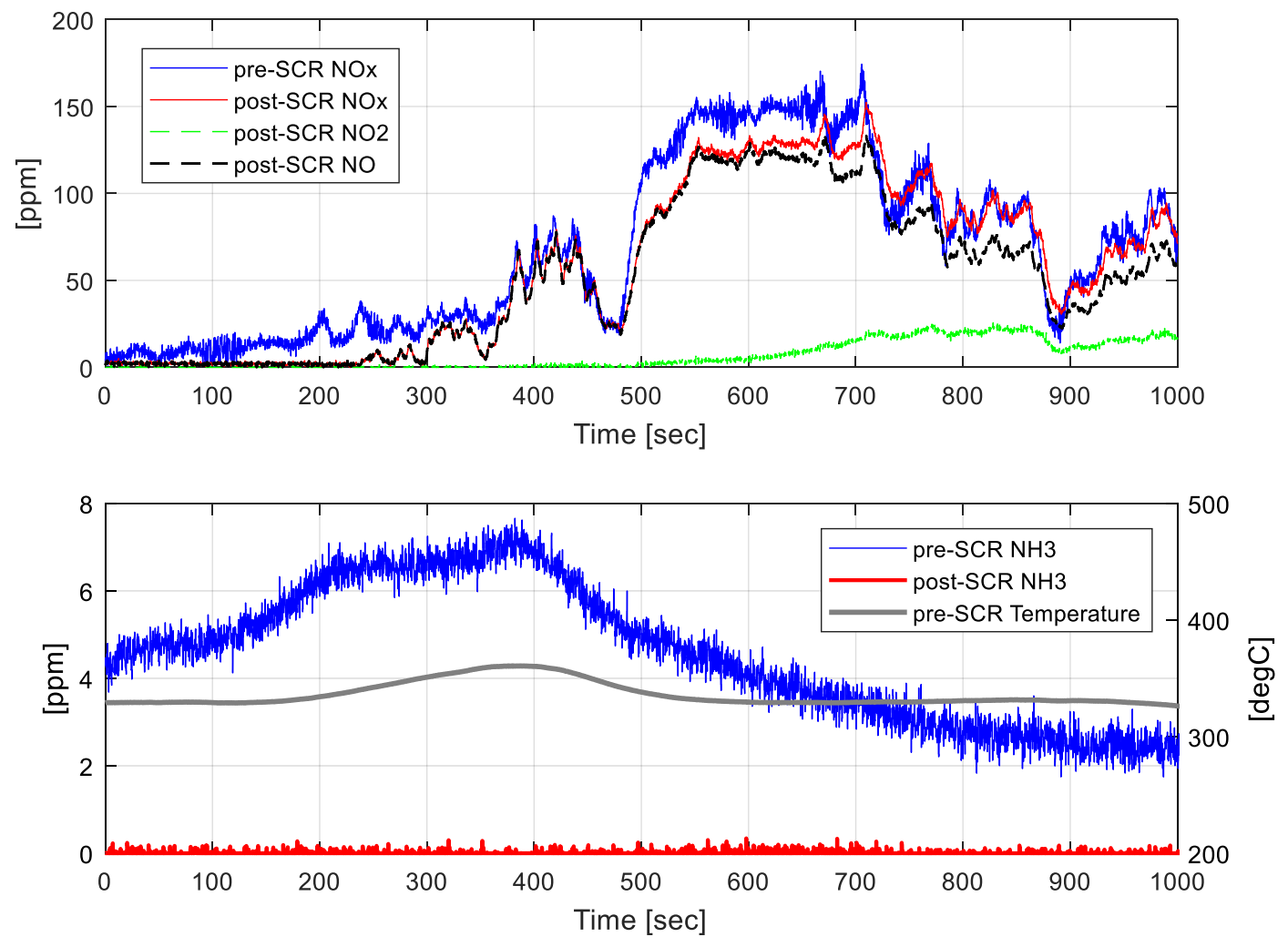

Figure 47: Mode 2 [engine speed $=851 \mathrm{rpm}$, engine torque $=84 \mathrm{ft}-\mathrm{lbs}$ ]; comparing pre-and-post $\mathrm{NOx}$ and $\mathrm{NH}_{3}$ concentrations; includes lean-ON period (480-700 seconds)

Figure 48, displays Mode 3, a mid-engine speed and high load condition resulting in higher pre-SCR temperature (on average of $509^{\circ} \mathrm{C}$ ), than compared to the previous two modes. This mode had a higher amount of $\mathrm{NH}_{3}$ entering the SCR catalyst, where the catalyst stored $\mathrm{NH}_{3}$ for about 530 seconds into the mode, and then $\mathrm{NH}_{3}$ desorption at observed based on the $\mathrm{NH}_{3}$ slippage from the SCR. It is also observed that the rate of ammonia being slipped is stable with an average value of $6 \mathrm{ppm}$ till 810 seconds into the mode, and starts rising with a faster rate to a peak of $203 \mathrm{ppm}$ in roughly 170 second duration. This release or desorption characteristics could be contributed to rise in SCR temperature, as can been depicted from the SCR inlet temperature provided by the grey line and the sudden rise is $\mathrm{NH}_{3}$ slippage could also be due to passing a certain desorption threshold which tend to depend on the catalyst surface temperature. After the $\mathrm{NH}_{3}$ peak observed at around 900 seconds, the rate of desorption drops at a slower pace till 15400 seconds and tend to stabilize in a cyclic behavior, with an average concertation of $80 \mathrm{ppm}$. NOx reduction was well observed with almost 100\% conversion by 
the catalyst during the baseline engine operation due to availability of enough stored $\mathrm{NH}_{3}$ in the catalyst. The lean-ON period was triggered at 2040 seconds and turned off at 2280 seconds into the mode. During the start of lean operating condition, the pre-SCR NOx quickly rises and stabilizes around $900 \mathrm{ppm}$, while we observe slow rise in the post-SCR NOx values due to mainly reduction reaction occurring between stored $\mathrm{NH}_{3}$ with $\mathrm{NO}$ and $\mathrm{O}_{2}$. No noticeable $\mathrm{NO}_{2}$ was seen during the entire mode 3, indicating negligible oxidation of either $\mathrm{NO}$ or $\mathrm{NH}_{3}$. The $\mathrm{NH}_{3}$ produced by the TWC also gradually drops in the beginning of the lean period to almost negligible trace of $\mathrm{NH}_{3}$. On the other hand, we also observe gradual reduction in $\mathrm{NH}_{3}$ slippage after the SCR, indicating that the NOx reduction is utilizing the stored $\mathrm{NH}_{3}$ for the conversion reactions. Similarly, the $\mathrm{NH}_{3}$ slippage goes to almost zero slip, and starts to absorb all the $\mathrm{NH}_{3}$ being produced by the TWC ones the lean operation was shut off. At around 2850 seconds, we notice another $\mathrm{NH}_{3}$ desorption and at around 2970 seconds when $\mathrm{NH}_{3}$ slippage reached roughly $27 \mathrm{ppm}$, based on visual inspection, the lean-ON was again switched on. In comparable manner, as the previous lean-ON period, NOx after the TWC increased but NOx outlet of the SCR did not increase till about 17 seconds, indicating 100\% reduction within this period. The entire mode 3 was ran for 3072 seconds at steady-state condition. Additionally, during the initial periods of the lean-ON operation, the temperature after the TWC increased with approximately $14^{\circ} \mathrm{C}$ difference, and then started dropping gradually over the period of the steady-state mode. The lean condition operating the engine in higher air-fuel ratio caused the exhaust temperature to slightly drop from the baseline condition.

Figure 49 presents mode 4, similar engine speed compared to the previous mode 3 but lower torque engine operating conditions. The mode is more comparable to mode 1 and mode 2, resulting in similar trend on $\mathrm{NOx}$ reduction., $\mathrm{NH}_{3}$ adsorption and in $\mathrm{NO}_{2}$ reaction in the SCR catalyst. No desorption of $\mathrm{NH}_{3}$ was observed for the tested steady-states duration. 

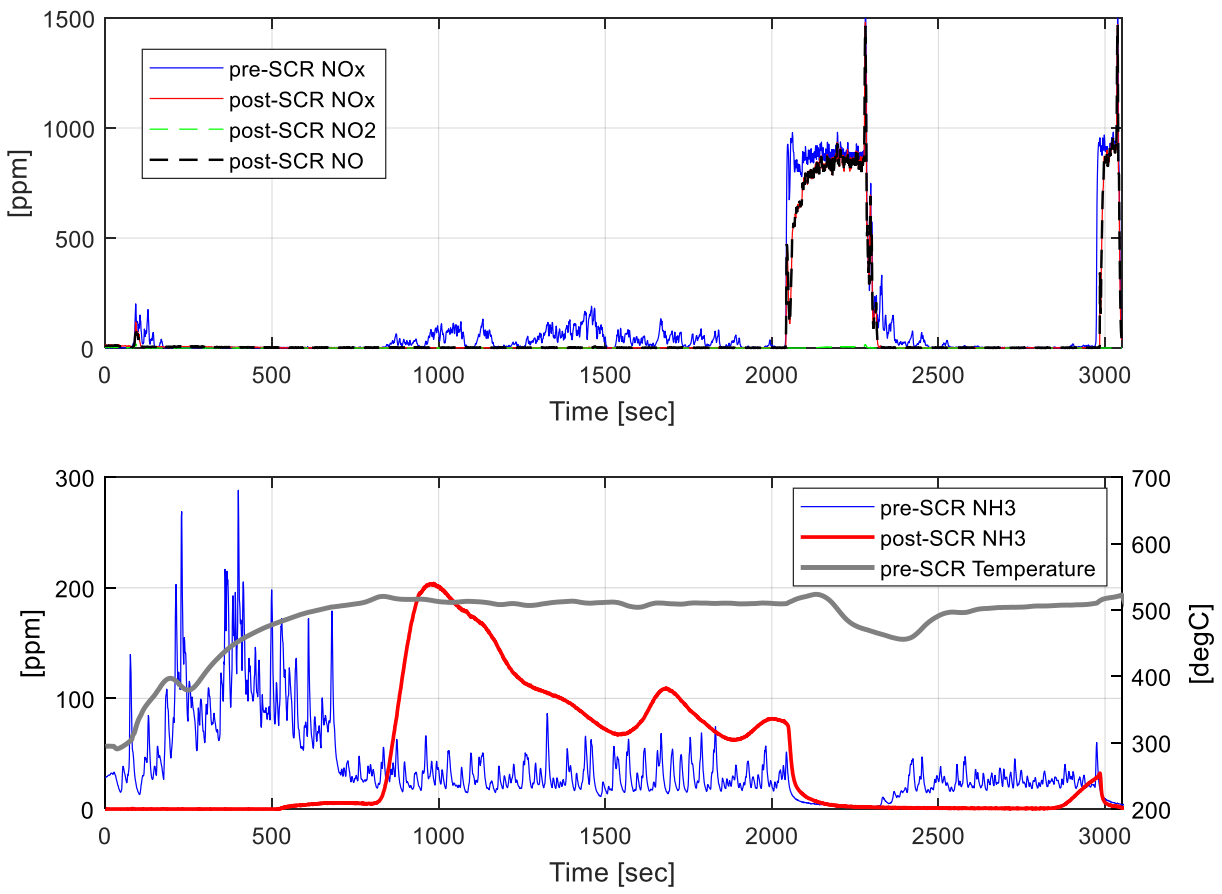

Figure 48: Mode 3 [engine speed $=1249 \mathrm{rpm}$, engine torque $=316 \mathrm{ft}-1 \mathrm{bs}$ ] $;$ comparing pre-and-post $\mathrm{NOx}$ and $\mathrm{NH}_{3}$ concentrations; includes two lean-ON periods (2040-2280 seconds \& 2970-3034 seconds)
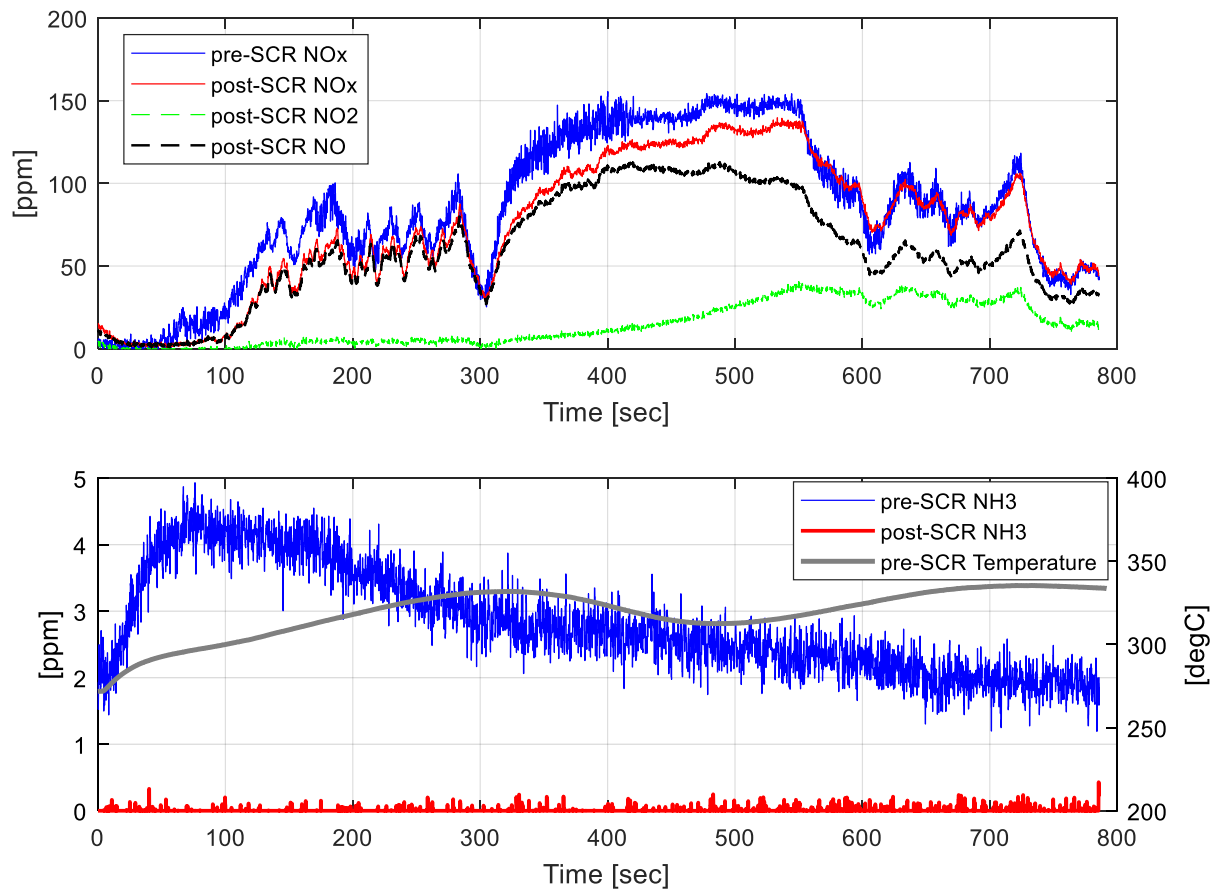

Figure 49: Mode 4 [engine speed $=1296 \mathrm{rpm}$, engine torque $=37 \mathrm{ft}-1 \mathrm{bs}$ ]; comparing pre-and-post $\mathrm{NOx}$ and $\mathrm{NH}_{3}$ concentrations; includes lean-ON period (310-550 seconds) 
Figure 50 shows mode 5 steady-state results, which consist of higher engine speed and load operating conditions. The average temperature before the SCR catalyst from the entire steady-state $572.6^{\circ} \mathrm{C}$ with a maximum temperature of $585^{\circ} \mathrm{C}$. It is to be noted that these elevated temperatures at steady conditions are usually not observed in real-world dynamic operation of the engine and only depicted under constant steady-state high load operation as performed for under this study. For this particular mode, the lean-ON period was switched on even before the observing any $\mathrm{NH}_{3}$ slippage after the SCR, in order to evaluate the NOx reduction with stored $\mathrm{NH}_{3}$ before any desorption. Right after switching the lean-ON period, the NOx after TWC indicated a sharp rise. We also observe the post-SCR NOx rise with minimum NOx reduction and quickly following the pre-SCR NOx levels. This was primarily done to understand if the SCR could be completely emptied (or regenerated) from the prior stored $\mathrm{NH}_{3}$ and initiate a condition with minimal ammonia storage ratio. Accordance to experimental reactor studies, researchers utilizes elevated temperature effect to completely desorb $\mathrm{NH}_{3}$ from the catalyst (Ciardelli et al. 2004). However, it becomes a challenge with the engine operations and experimental setup where $\mathrm{NH}_{3}$ dynamics and temperature after the TWC cannot be directly controlled or held constant.

In elaborating further for mode 5 , in Figure 50 , traces of $\mathrm{NH}_{3}$ desorption was observed at 345 seconds into the mode and increased rapidly with respect to the increasing temperature. The post-SCR $\mathrm{NH}_{3}$ slippage peaked at approximately $60 \mathrm{ppm}$ and 410 seconds into the mode, roughly taking 65 seconds from detecting $\mathrm{NH}_{3}$ slip to maximum slippage concentration. After the $\mathrm{NH}_{3}$ peak, gradual drop in $\mathrm{NH}_{3}$ slippage was observed and reached a stabilizing concentration within (20-25) ppm. The second lean-ON was switched on at 774 seconds into the mode, causing the pre-SCR $\mathrm{NH}_{3}$ to drop along with the post-SCR which could be attributed to the availability of $\mathrm{NH}_{3}$ storage instigating reduction reaction at high $\mathrm{NOx}$ and oxygen levels. The lean operation was then turned off at around 1010 seconds into the mode. From the $\mathrm{NH}_{3}$ slippage trace, $\mathrm{NH}_{3}$ started to desorb at a higher rate after 90 seconds into turning the lean operation off and stabilizing around the same level as then let $\mathrm{NH}_{3}$, approximately (20-25) ppm. The mode showed no indication of $\mathrm{NO}_{2}$ formation in the SCR catalyst, even during the leanON periods as observed in the previous modes. 

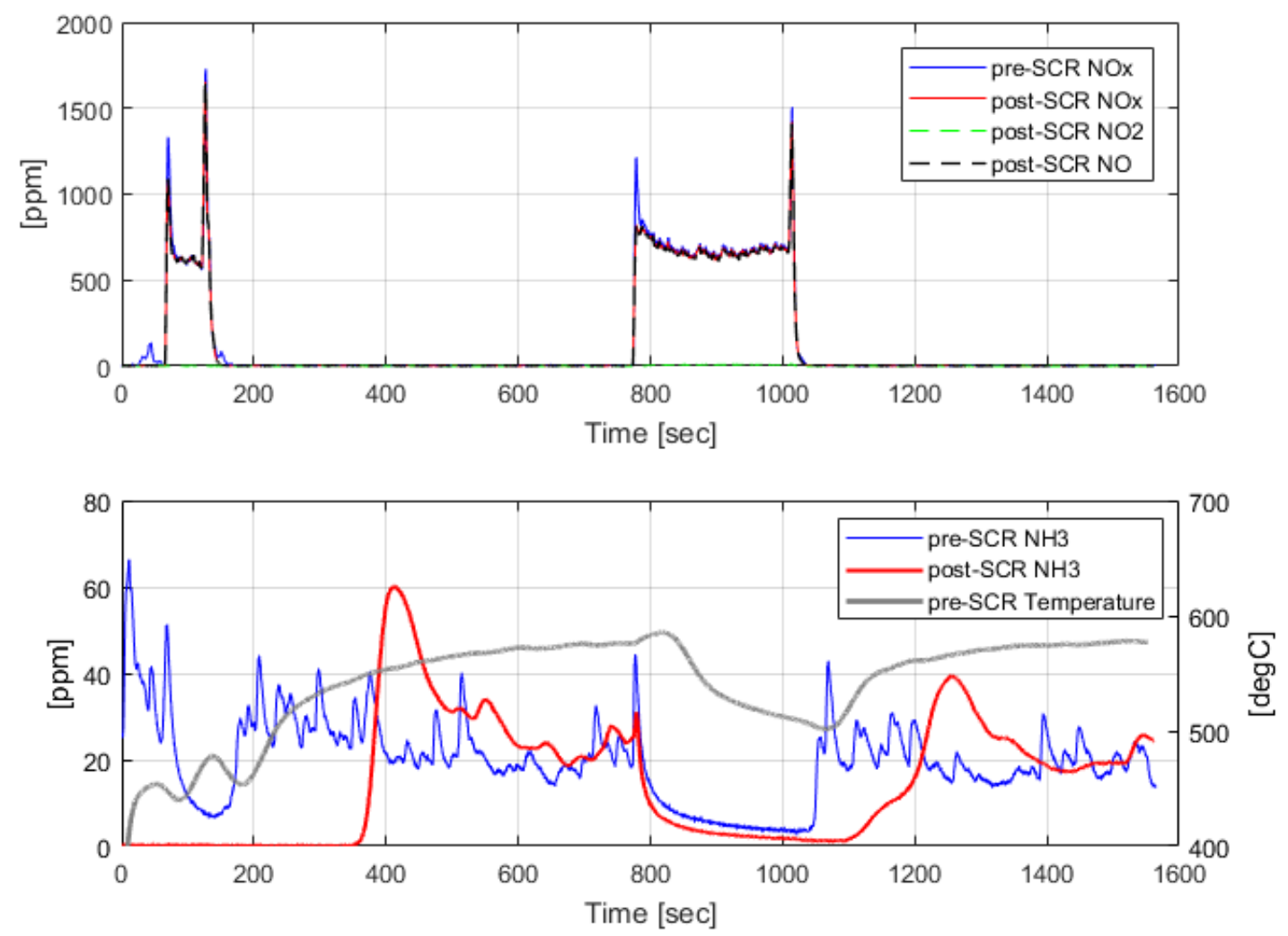

Figure 50: Mode 5 [engine speed $=1480 \mathrm{rpm}$, engine torque $=597 \mathrm{ft}-1 \mathrm{bs}$ ] $;$ comparing pre-and-post $\mathrm{NOx}$ and $\mathrm{NH}_{3}$ concentrations; includes two lean-ON periods $(65-122$ seconds \& 774-1010 seconds)

Figure 51 present mode 6, a high engine speed but low load operating condition. The average temperature for the mode was more stable at approximately $468.2^{\circ} \mathrm{C}$. It can be observed that almost all the NOx entering the SCR is being reduced by the catalyst reaction during the baseline operation period of the mode. For this mode, the lean-ON was switched on for just brief periods. The first lean-ON was switched on at 412 seconds for only 7 seconds while the second lean-ON was switched on at 460 seconds but for a longer duration, i.e. 19 seconds. Comparing the pre-and-post NOx traces for the two lean-ON periods, the first lean-ON indicated that the duration was short enough to avoid the post-SCR NOx peak as seen in the second lean-ON period. However, the second lean-ON period with 19 seconds of duration was enough to result in decreasing the $\mathrm{NH}_{3}$ slippage trend as seen by the red curve around 460 seconds on the bottom chart of Figure 51. Evaluation of different lean-ON periods on $\mathrm{NH}_{3}$ slippage rates and NOx conversion control are presented in Section 5.3.2. 

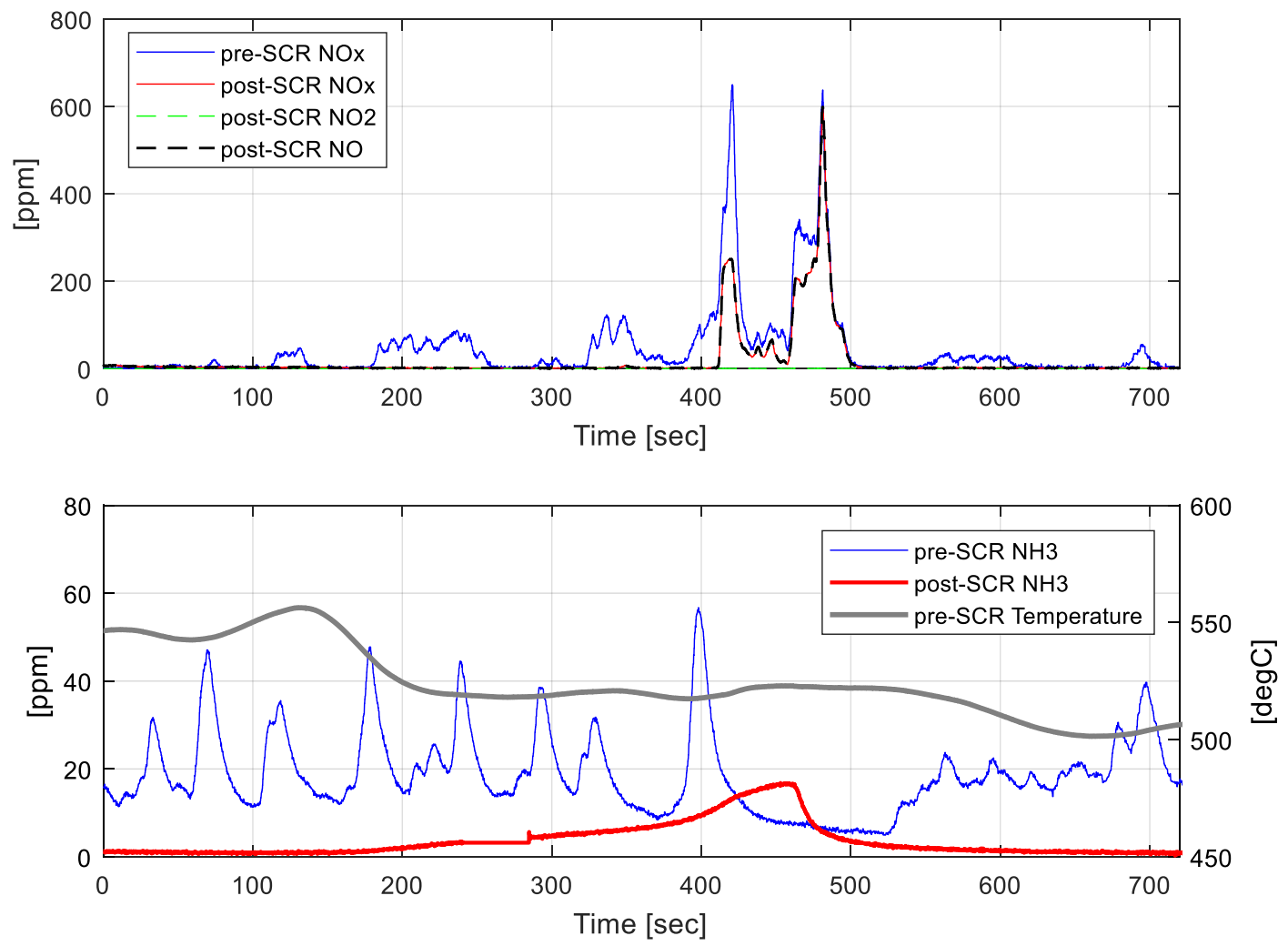

Figure 51: Mode 6 [engine speed $=1521 \mathrm{rpm}$, engine torque $=162 \mathrm{ft}-\mathrm{lbs}$ ]; comparing pre-and-post $\mathrm{NOx}$ and $\mathrm{NH}_{3}$ concentrations; includes two lean-ON periods (412-419 seconds \& 460-479)

Figure 52 and Figure 53 displays mode 7 and mode 8, with similar high engine speed operations but with two different load levels. Mode 8 operated at higher torque value producing the maximum power among all other modes. Both modes showed similar characteristics in $\mathrm{NH}_{3}$ adsorption and desorption rates during baseline and lean-ON periods. However, during the first lean-ON period in mode $7, \mathrm{NO}_{2}$ formation was also observed after SCR (shown by the green dashed lines). Investigating this further, it was observed that the temperatures of the inlet and outlet of the SCR catalyst were at lower ranges $\left(445^{\circ} \mathrm{C}\right.$ at the inlet and $264{ }^{\circ} \mathrm{C}$ at the outlet; averaged temperatures during the lean-ON periods) and additional attributed to potential oxidation reaction aiding $\mathrm{NO}_{2}$ formation in the SCR catalyst. No noticeable $\mathrm{NO}_{2}$ was observed in any of the three lean-ON periods in the mode 8 test. 

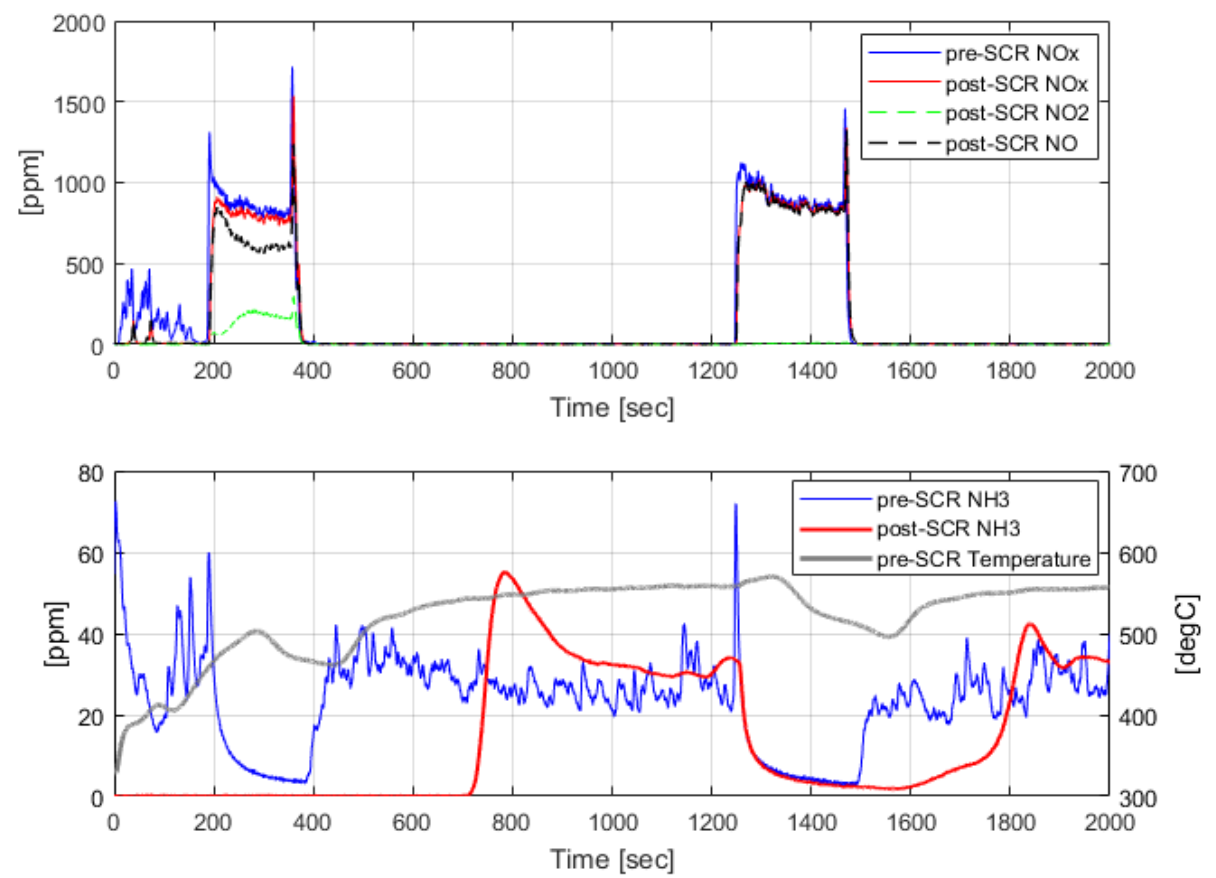

Figure 52: Mode 7 [engine speed $=1575 \mathrm{rpm}$, engine torque $=337 \mathrm{ft}-1 \mathrm{bs}$ ] ; comparing pre-and-post NOx and $\mathrm{NH}_{3}$ concentrations; includes two lean-ON periods (182-789 seconds \& 1245-1464 seconds)
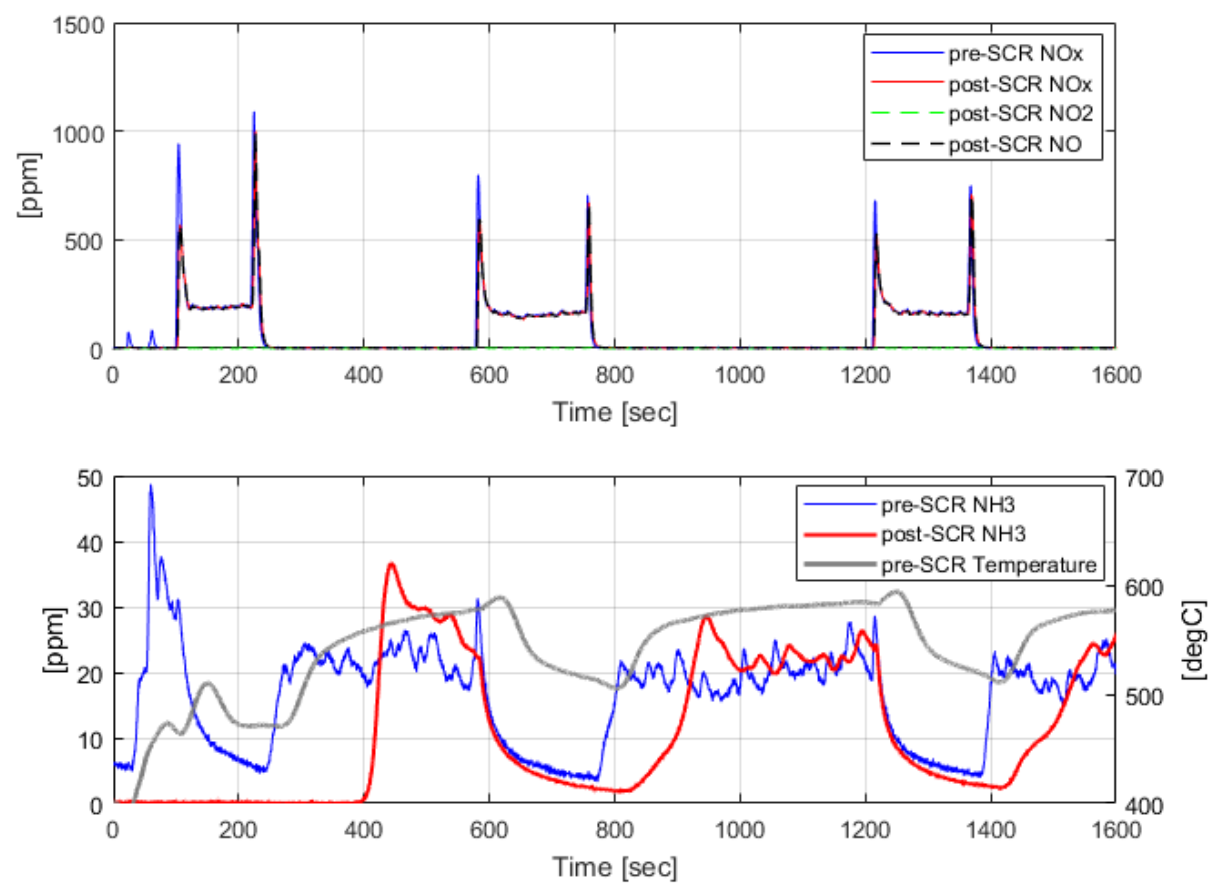

Figure 53: Mode 8 [engine speed $=1611 \mathrm{rpm}$, engine torque $=683 \mathrm{ft}-\mathrm{lbs}$ ]; comparing pre-and-post NOx and $\mathrm{NH}_{3}$ concentrations; includes three lean-ON periods (90-219 seconds, 577-752 seconds \& $1211-1362$ seconds) 


\subsubsection{Engine performance evaluation during lean-ON Modes}

This section presents results comparing the baseline versus the lean-ON engine operation obtained from previous stead-state experiments. Table 10, summarizes the difference in power and carbon-based components obtained from the CVS iterative carbon balance method averaged over two engine operating conditions for all 8 modes along.

Table 10. Summary comparison between baseline operation versus lean operation over eight steady-state modes

\begin{tabular}{|c|c|c|c|c|c|c|c|}
\hline $\begin{array}{l}\text { Steady- } \\
\text { State } \\
\text { Mode } \\
\end{array}$ & $\begin{array}{l}\text { Engine } \\
\text { Operation }\end{array}$ & $\begin{array}{c}\text { Average } \\
\text { Power } \\
\text { [bhp] }\end{array}$ & $\begin{array}{c}\text { Power } \\
\text { COV }\end{array}$ & $\begin{array}{c}\text { Fuel CB } \\
\text { [g/bhp- } \\
\text { hr] }\end{array}$ & $\begin{array}{c}\mathrm{CO}_{2} \\
{[\mathrm{~g} / \mathrm{bhp}-} \\
\mathrm{hr}]\end{array}$ & $\begin{array}{c}\mathrm{CO} \\
{[\mathrm{g} / \mathrm{bhp}-} \\
\mathrm{hr}]\end{array}$ & $\begin{array}{c}\text { THC } \\
\text { [g/bhp- } \\
\text { hr] }\end{array}$ \\
\hline \multirow{3}{*}{1} & BASE & 35.6 & 0.1 & 135.4 & 366.4 & 0.05 & 3.61 \\
\hline & Lean-ON & 33.0 & 0.3 & 133.5 & 362.1 & 0.05 & 3.23 \\
\hline & $\%$ Difference & $7.5 \%$ & & $1.4 \%$ & $1.2 \%$ & $-0.2 \%$ & $10.6 \%$ \\
\hline \multirow{3}{*}{2} & BASE & 13.2 & 0.3 & 219.6 & 602.0 & 0.00 & 3.12 \\
\hline & Lean-ON & 12.9 & 0.4 & 212.8 & 579.0 & 0.00 & 4.55 \\
\hline & $\%$ Difference & $2.3 \%$ & & $3.1 \%$ & $3.8 \%$ & $0.0 \%$ & $-45.7 \%$ \\
\hline \multirow{3}{*}{3} & BASE & 76.2 & 0.8 & 132.6 & 367.1 & 0.06 & 0.53 \\
\hline & Lean-ON & 76.6 & 1.7 & 129.1 & 355.1 & 0.04 & 1.41 \\
\hline & $\%$ Difference & $-0.5 \%$ & & $2.6 \%$ & $3.3 \%$ & $30.1 \%$ & $-167.4 \%$ \\
\hline \multirow{3}{*}{4} & BASE & 9.6 & 0.1 & 376.9 & 1028.4 & 0.00 & 7.11 \\
\hline & Lean-ON & 9.2 & 0.2 & 381.2 & 1039.5 & 0.00 & 7.43 \\
\hline & $\%$ Difference & $4.7 \%$ & & $-1.1 \%$ & $-1.1 \%$ & $0.0 \%$ & $-4.5 \%$ \\
\hline \multirow{3}{*}{5} & BASE & 168.9 & 1.1 & 127.7 & 354.0 & 0.65 & 0.06 \\
\hline & Lean-ON & 169.5 & 4.0 & 123.8 & 341.4 & 0.02 & 0.97 \\
\hline & $\%$ Difference & $-0.4 \%$ & & $3.1 \%$ & $3.5 \%$ & $96.4 \%$ & $-1395.2 \%$ \\
\hline \multirow{3}{*}{6} & BASE & 45.3 & 0.3 & 160.3 & 444.3 & 0.46 & 0.27 \\
\hline & Lean-ON & 37.4 & 0.5 & 183.1 & 505.7 & 0.04 & 1.24 \\
\hline & $\%$ Difference & $17.6 \%$ & & $-14.2 \%$ & $-13.8 \%$ & $90.3 \%$ & $-358.7 \%$ \\
\hline \multirow{3}{*}{7} & BASE & 100.8 & 0.4 & 140.8 & 390.3 & 0.18 & 0.32 \\
\hline & Lean-ON & 100.8 & 0.8 & 135.6 & 374.5 & 0.00 & 0.92 \\
\hline & $\%$ Difference & $0.1 \%$ & & $3.7 \%$ & $4.1 \%$ & $99.9 \%$ & $-186.5 \%$ \\
\hline \multirow{3}{*}{8} & BASE & 207.9 & 1.4 & 125.9 & 348.4 & 0.91 & 0.06 \\
\hline & Lean-ON & 207.4 & 6.2 & 123.7 & 340.1 & 0.01 & 1.41 \\
\hline & $\%$ Difference & $0.3 \%$ & & $1.7 \%$ & $2.4 \%$ & $99.4 \%$ & $-2252.1 \%$ \\
\hline
\end{tabular}

Comparing the average power between the two engine operations, baseline and leanON, we observe some higher difference, especially at lower engine operation modes. During the steady-state modes, the dynamometer control and the engine were manually operated via throttle controller manner to reach the desired engine power, the engine switching to leaner operation caused the power to drop for the same set throttle position. The throttle was then adjusted to match the intended power levels under stable engine conditions. For some modes, it was not possible to hold at the specific torque value due to high torque fluctuation and the 
engine's response to the throttle control resolution (or step in throttle position). An example of such mode would be mode6, where a difference of $17.6 \%$ is observed between the baseline and the lean-ON operation. On the other hand, in the event of lean-ON periods, the engine did exhibit higher torque fluctuation than compared to the stochiometric baseline operation, and the level of fluctuation can be interpreted based on observing the coefficient of variation (COV). The COV for the lean operation were observed to be higher than the baseline operation and the cause in such variation in the engine torque stabilization could depended upon different combustion operating parameters, such as, fuel quantity, dithering profiles and/or spark timings. The study did not have access or control over such engine parameters, and further work needs to be done to understand the effects of engine calibration under the lean-ON operation performed for the 8 steady-state modes. The table also provides brake specific fuel consumption during each steady state operation calculated using the carbon balance method from the CVS measurement system.

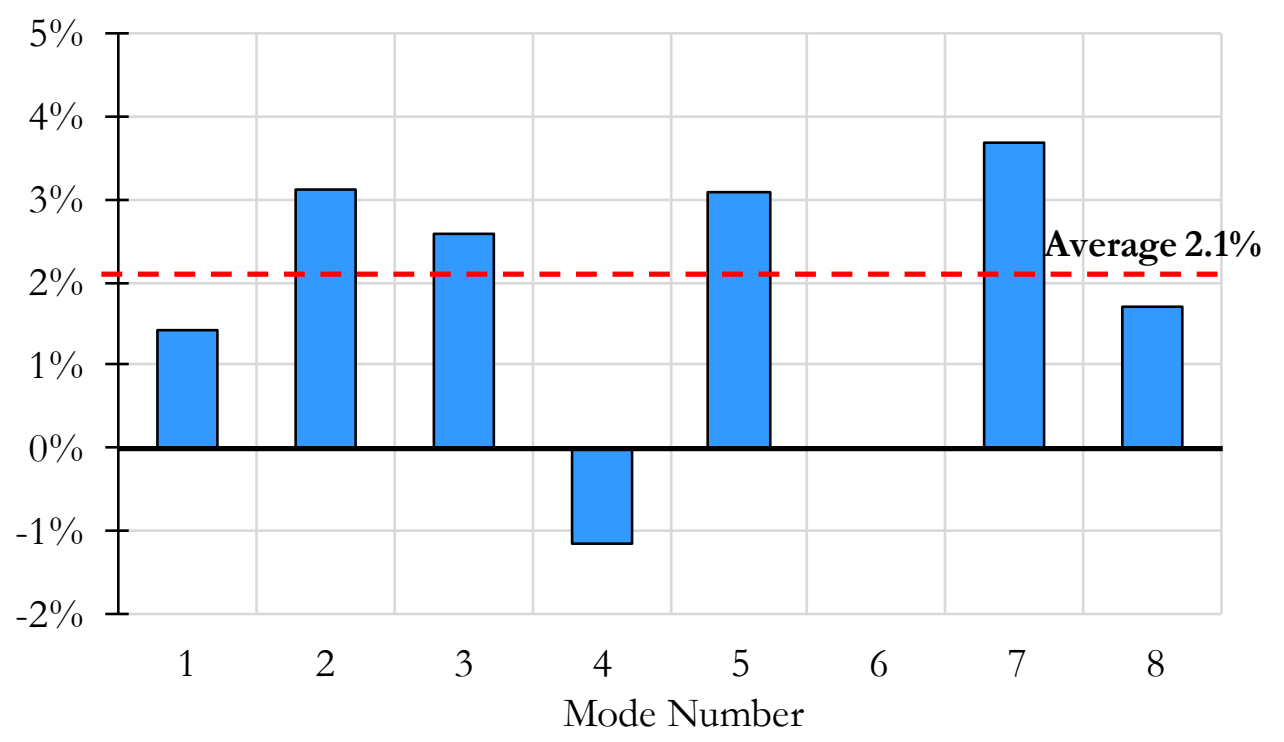

Figure 54. Comparison of brake specific fuel consumption between baseline operation versus lean-ON operation

The graphical representation of the percent difference comparison between the baseline and lean-ON operation is provided in Figure 54. Due to high power differences observed in mode 6 , the fuel consumption value was excluded in calculating the overall average percent difference between the two operating modes. By operating the engine into leaner condition 
under the same power levels resulted on average of $2.1 \%$ lower brake specific fuel consumption. A maximum fuel benefit of $3.7 \%$ was observed in mode 7 .

On the other hand, $\mathrm{CO}$ emissions were significantly lower, especially at higher engine power modes, when operating the engine in lean operation. Differences of up to $90 \%$ and above were observed for modes (5, 6, 7 and 8). However, the comparisons also indicate significantly higher THC emissions at these modes in lean-ON operations. It is to be noted that, both $\mathrm{CO}$ and THC values are direct consequence of TWC performance and engine out exhaust conditions due to operating the engine under lean combustion, and the SCR did not have any effect in the change of these two species.

\subsubsection{Lean-ON duration evaluation}

It has been observed that switching the lean-ON operation changes the TWC output $\mathrm{NOx}$ and $\mathrm{NH}_{3}$ characteristics and this simultaneously affects the dynamics of $\mathrm{NH}_{3}$ adsorption and NOx reduction characteristics of the passive-SCR system. Additionally, amount of NOx that can be reduced by the SCR catalyst depends up the stored $\mathrm{NH}_{3}$, as the surface reaction of $\mathrm{NOx}$ reduction happens over the catalyst with prior $\mathrm{NH}_{3}$ adsorbed. Hence, the duration of the lean-ON period significantly plays a big role in both $\mathrm{NOx}$ reduction and $\mathrm{NH}_{3}$ regeneration process during dynamic conditions.

Figure 55 shows results of conducting a steady-state mode (Mode-3) with different leanON duration and the effect on the SCR performance. The lean-ON periods were manually triggered to start and stop. The duration of the lean-ON period was further calculated based on the rise and fall of the oxygen sensor signal which indicated the engine's response in being operated under lean-operation. The chart depicts nine lean-ON periods and all points were triggered when there was noticeable $\mathrm{NH}_{3}$ slip after the SCR catalyst. Lean-ON with less than 2 seconds period did not gave any change in the steady-state operation in terms of $\mathrm{NOx}$ and $\mathrm{NH}_{3}$ from the TWC. However, increasing the lean-ON period for above 2 seconds showed increase in the TWC NOx, indicated by the red diamond points $(\Delta)$, with highest (1919 ppm) observed approximately 7 seconds. The blue circles indicate average NOx reduction during the lean-ON periods and higher reduction are observed between (2 to 3) seconds of lean-ON periods. It also shows that as we go higher in duration the overall NOx reduction decreases, since the maximum NOx reduction mainly occurs during the initial rise of the NOx when NOx molecules comes in direct contact with stored $\mathrm{NH}_{3}$ and unreacted $\mathrm{NOx}$ tends to slip through the catalyst. On the 
other hand, decrease in $\mathrm{NH}_{3}$ slippage response after the lean-ON trigger were only observed for higher duration events. The points with solid fills indicate the downward trend in post SCR $\mathrm{NH}_{3}$ concentration, followed by the consequences of the lean-ON respective lean-ON periods. Out of all events, the duration with 3 seconds lean-ON period resulted in 25\% NOx reduction and forced a downward trend in $\mathrm{NH}_{3}$ slip. Evaluating reactions mechanism in molar balance will better indicate the duration needed for complete regeneration. In summary, the ammonia storage ration becomes an important parameter in control-oriented model for SCR regeneration using lean operation events. For simplicity, the study selects 3 seconds for the short-duration and 4 seconds for the long-duration triggers, to be used by the fuzzy logic regen trigger output variable.

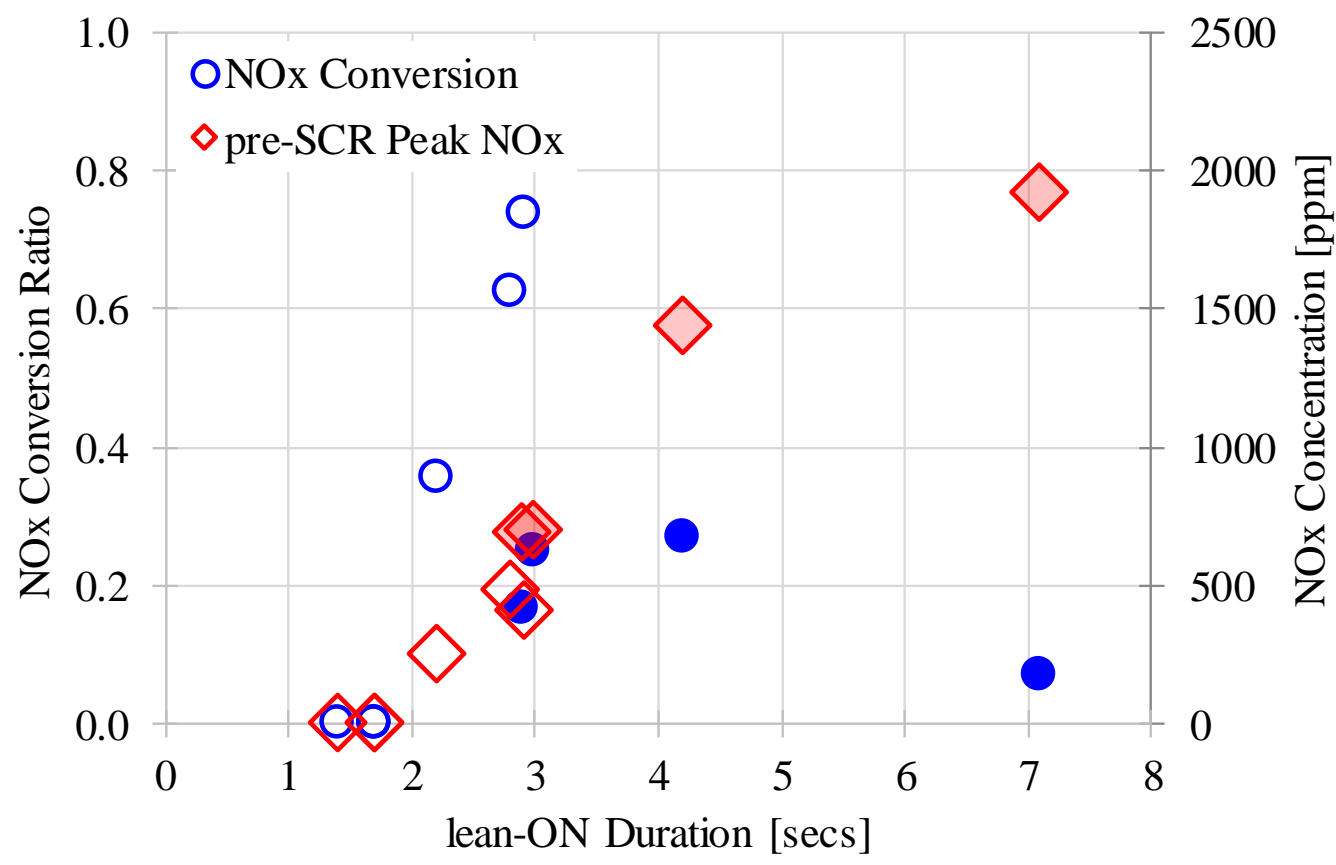

Figure 55. Comparison of varying lean-ON periods (Mode 3)

\subsection{Regeneration control using Fuzzy-Logic}

An inference rule based fuzzy logic controller which required two sensor signals as input variables in order to initiate regeneration trigger modes were developed and presented in this section. Ranges for each membership function were chosen based on experimental data for the observed values of $\mathrm{NH}_{3}$ and $\mathrm{NOx}$ after the SCR catalyst. Figure 56 presents the membership function for each three fuzzy inputs used in designing the fuzzy logic controller. The size of 
each membership function within each defined linguistic variable varies based on the desired control within the data range. Finer control of the ammonia slip is expected at lower range for observed $\mathrm{NH}_{3}$ slip and potentially regen events. The control avoids large slip of $\mathrm{NOx}$ and, hence, small slip levels of rules are given to maintain excessive NOx slip. In order to avoid large variation in calculated rate of ammonia slip (RAS) due to fluctuation in the measured concentration, it was calculated on the larger time scale observing the difference between the current and prior concertation at 5 seconds of time interval.
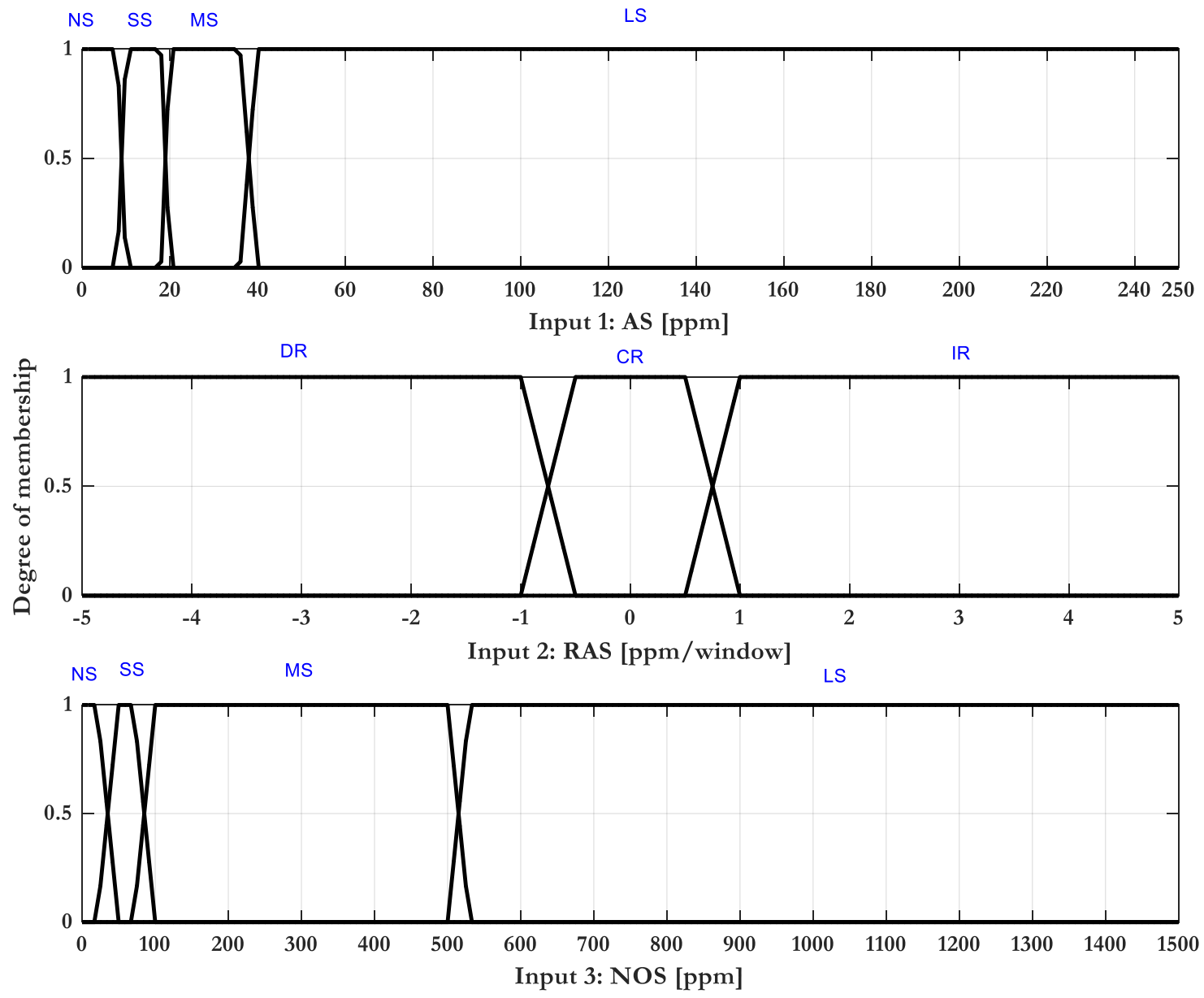

Figure 56. Ammonia Slip (AS), Rate of Ammonia Slip (RAS), and NOx Slip (NOS) Membership Function

Figure 57, Figure 58 and Figure 59 presents the controller response when making uses of membership function and inference rules developed fuzzy logic controller. Since, it was not possible to model the combined (engine + TWC + SCR) system response due to complexity of 
the system behavior, controller performance was investigated qualitatively against different obtained test data from the steady-state and transient experiments. The charts show the fuzzylogic controller making regeneration triggers smoothly over $\mathrm{NH}_{3}$ slip and turning off in the events of high $\mathrm{NOx}$ slip or low $\mathrm{NH}_{3}$ slip with decreasing rate of slip. The highlighted region shows the area where the controller commands the regeneration state to be $\mathrm{ON}$. The $\mathrm{y}$-axis on the right indicates the fuzzy output obtained from using middle of maximum method in the membership function leading to crisp output of either be at regen-OFF, regen-ON with shortdithering (0.625 fuzzy output), and on with long dithering (0.875 fuzzy output) modes. It's well observed that lean-ON periods would change the SCR behavior and the dynamic behavior of very test would completely be different if the engine was operated with the fuzzy-logic controller, based on feedback obtained from the two sensors. Additionally, the controller is a time-based looped controller, meaning the control would turn on the regen event for a fixed duration, either (SD or LD) and then operate back to a feedback loop observing the sensor values.

The fuzzy-logic control was not validated on an actual test experiment, and hence, the true nature of the controller performance on SCR regeneration would be inconclusive. The controller needs to be tested, evaluated and fine-tuned with the help of hardware-in-loop (H-IL) setup which can be powered by the Arduino-DUE board as built in this study for the manual lean-ON evaluation. The author recommends future validation for complete evaluation of the controller performance over real-world conditions. This method can be seen as a viable choice in stoichiometric natural gas engine operation to further meet fuel and emission standards with scope of lean operation regime. 

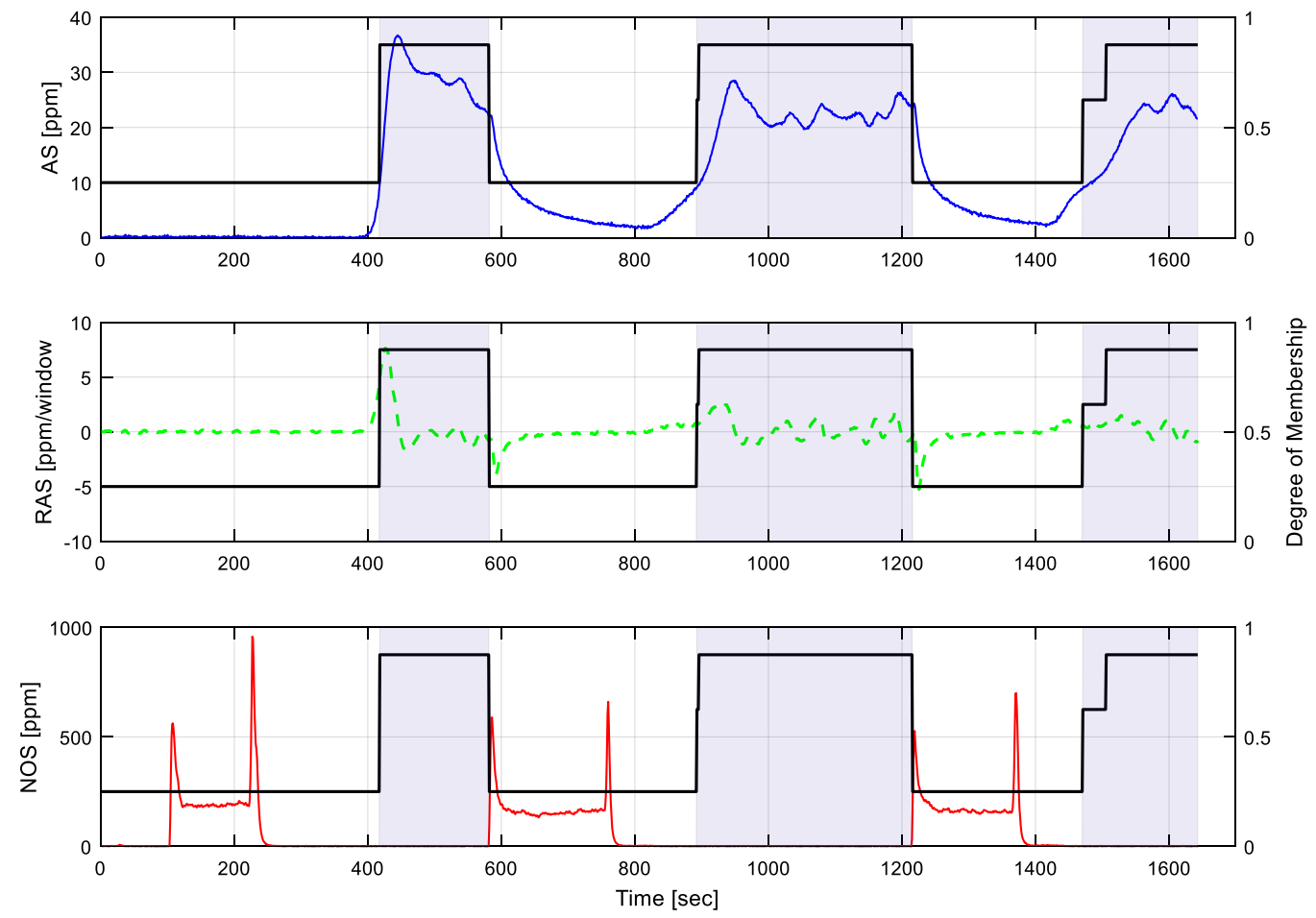

Figure 57. Fuzzy-logic controller response for steady-state Mode 8 (SCR 4)
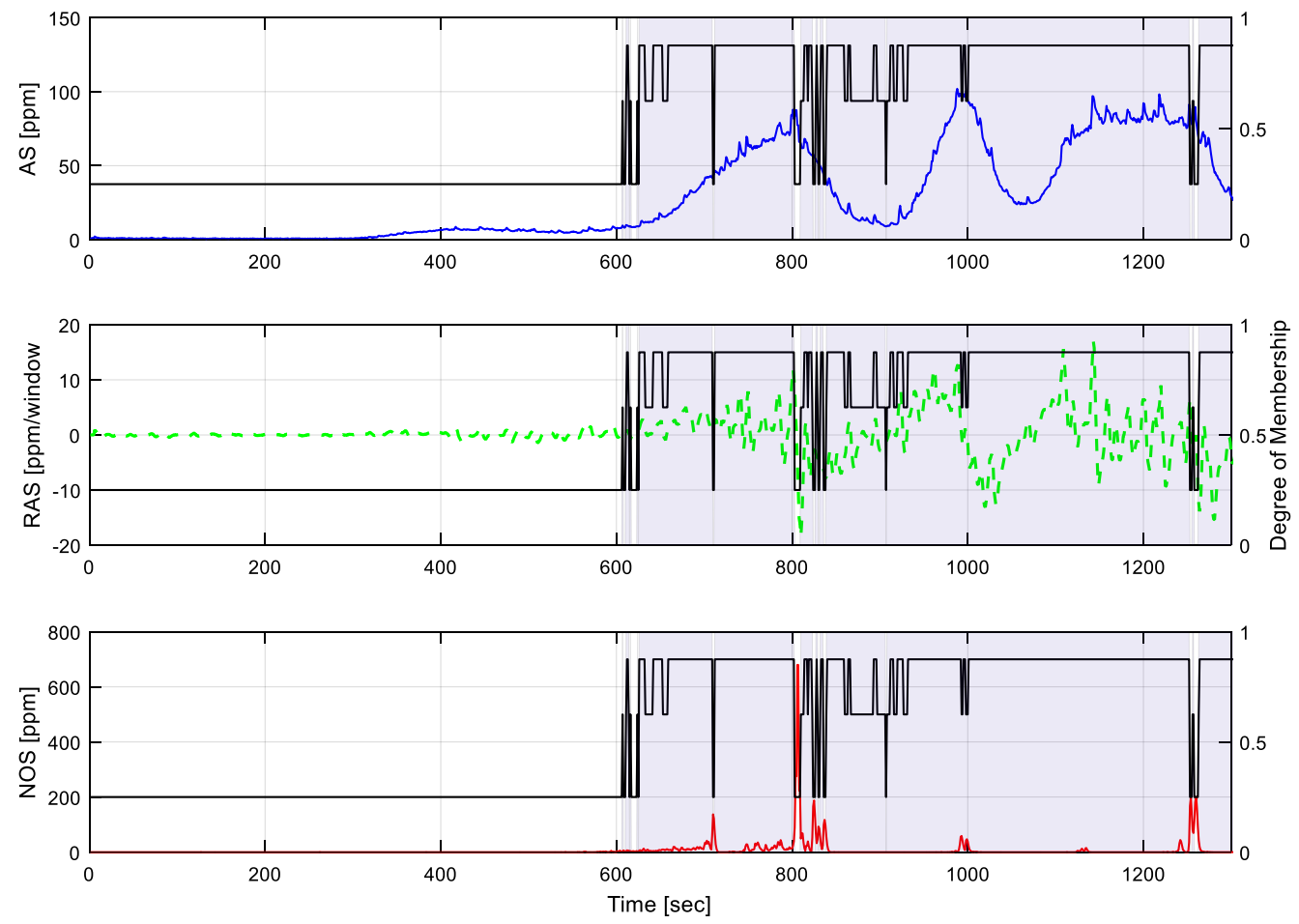

Figure 58. Fuzzy-logic controller response for steady-state Mode 3 (SCR 4) 

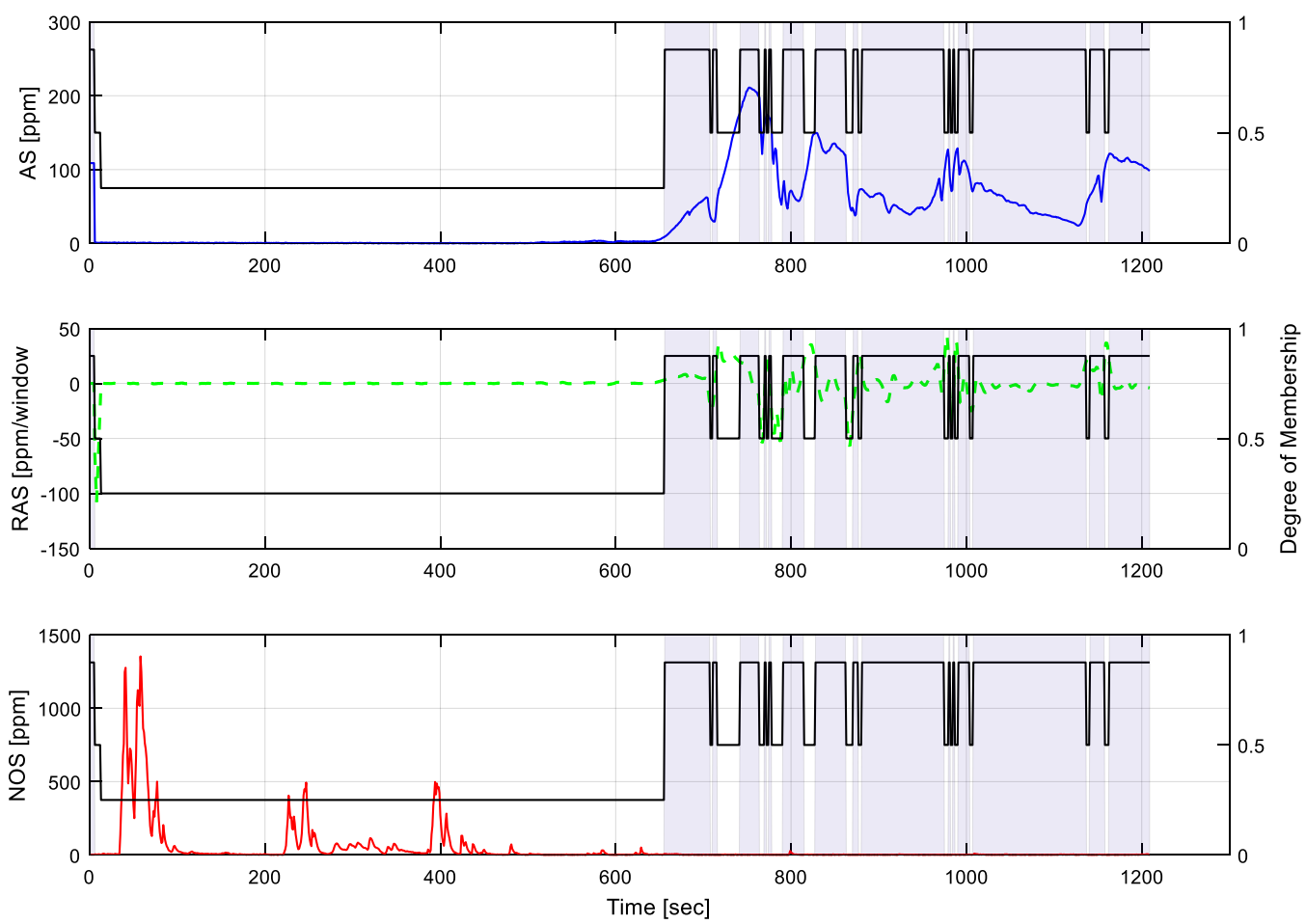

Figure 59. Fuzzy-logic controller response for transient FTP cycle (SCR 3) 


\subsection{Post-TWC $\mathrm{NH}_{3}$ estimation using ANN}

This section summarizes the results obtained from utilizing ANN technique to estimate post-TWC $\mathrm{NH}_{3}$ concentration using different engine parameters as training inputs. For the input data set, two combined transient FTP tests were used to train the model initially with seven different inputs and the measured $\mathrm{NH}_{3}$ concentration as the network output. The data set consisted of 23,980 data points which is equivalent to 23,980 seconds of the transient cycle measured. Figure 60 presents the input profile for all seven inputs used in training and evaluating the AN-N model.
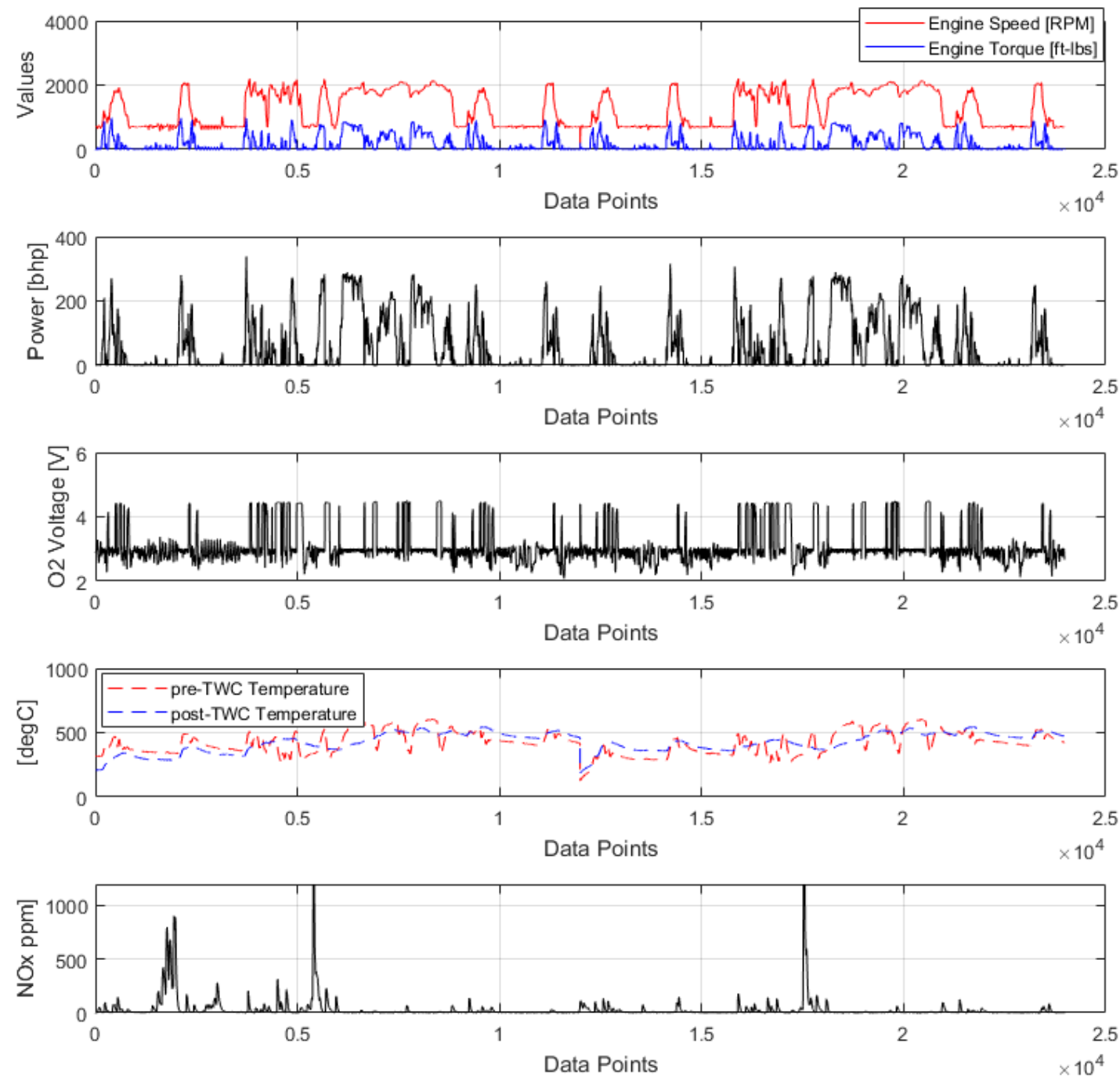

Figure 60. Transient FTP profile of the seven input variables 
The selected network was trained with different network properties and resulted were evaluated based on the estimated performance of the output parameter, i.e. $\mathrm{NH}_{3}$ concertation after the TWC. Table 11 and Table 12, presents results obtained from training the input-output network using two different training functions (Levenberg-Marquardt and Bayesian Regularization). The tables also include using these training function with different number of neurons. The results clearly show that with increasing neurons, we get better estimation of the output, provided by the R-squared number (indicates how the close the data fitted to a linear regression line) and Mean Square Error (MSE) number (indicates the quality of the estimation). However, with computational time to train the network increases due to added neurons through the network. It is also known that increasing the neurons increases the possibility of over training and over fitting the dataset, and to avoid certain circumstances, the errors were compared between the training, validation and test performances during each training iterations. Further training would stop if six consecutive errors would increase between the trained and validated errors. When comparing the performance between the two training functions, Bayesian Regularization provide slightly better performance, especially at lower number of neurons. The computational time for this function was significantly higher than LevenbergMarquardt.

Table 11. Comparison of performance using different number of neurons and Levenberg-Marquardt training function

\begin{tabular}{|r|c|c|c|c|}
\hline Number of Neurons & 10 & 20 & 30 & 40 \\
\hline R-squared & 0.799 & 0.850 & 0.899 & 0.916 \\
\hline Overall MSE & 578.4 & 422.2 & 278.3 & 235.2 \\
\hline Number of Iterations & 50 & 68 & 134 & 109 \\
\hline Training Time [sec] & 2.8 & 7.9 & 32.5 & 36.9 \\
\hline
\end{tabular}

Table 12. Comparison of performance using different number of neurons and Bayesian Regularization training function

\begin{tabular}{|r|c|c|c|c|}
\hline Number of Neurons & 10 & 20 & 30 & 40 \\
\hline R-squared & 0.844 & 0.889 & 0.902 & 0.916 \\
\hline Overall MSE & 432.6 & 301.0 & 272.9 & 231.4 \\
\hline Number of Iterations & 300 & 300 & 300 & 300 \\
\hline Training Time [sec] & 16.0 & 40.7 & 64.5 & 104.5 \\
\hline
\end{tabular}


Figure 61 and Figure 62, shows the estimated continuous traces of the $\mathrm{NH}_{3}$ concentration for Levenberg-Marquardt and Bayesian Regularization training functions, respectively. The figures also provide the residual charts for visualization differences between the actual and the measured. Examining the continuous traces, we see that the estimated $\mathrm{NH}_{3}$ concentration follows well with the actual measured curves (shown by the black line), but with observed noise. The trained network was able estimate better for the first few peaks seen from the profiles, especially when using 40 number of neurons (as depicted from the residual plots).
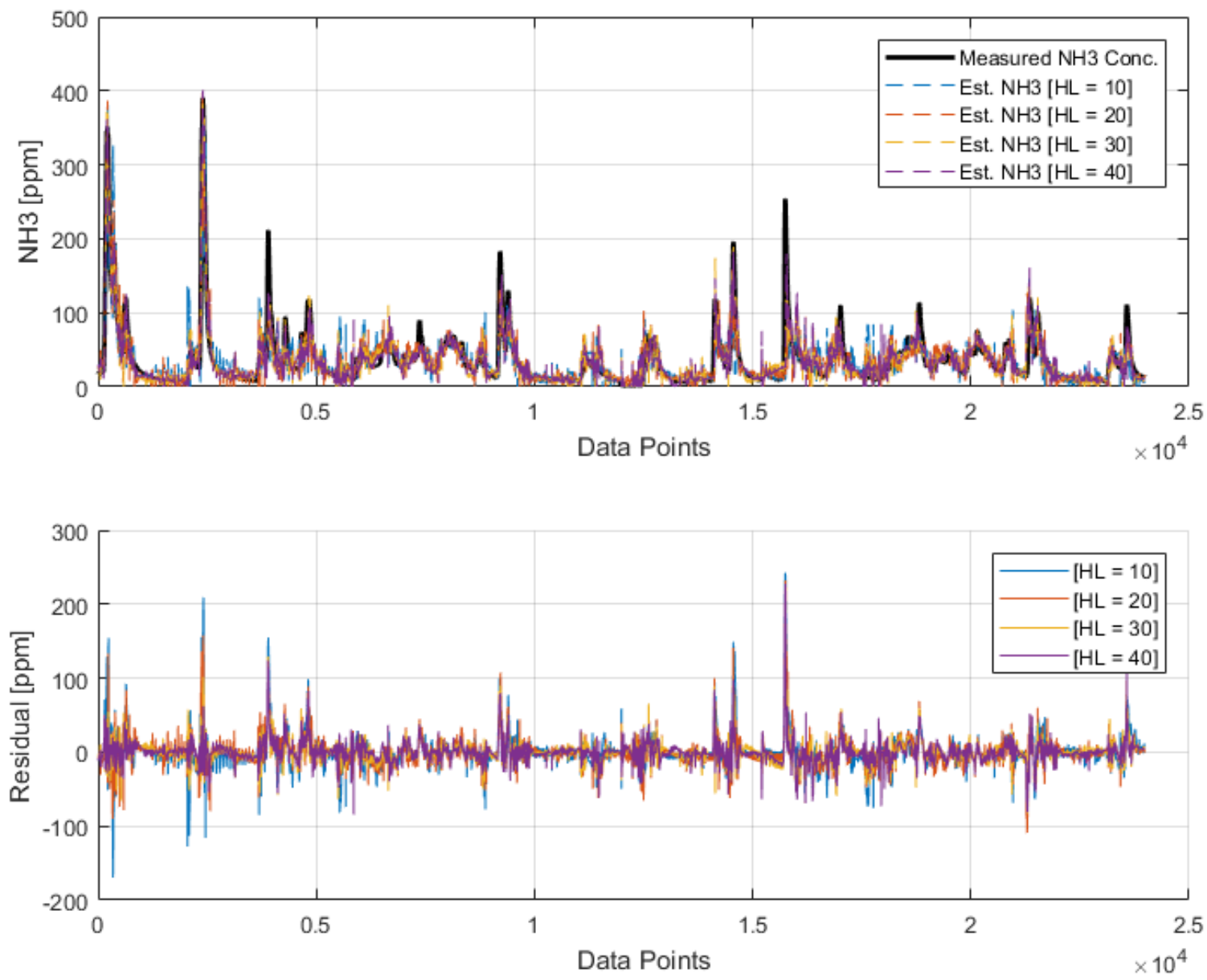

Figure 61. Results for measured versus estimated $\mathrm{NH}_{3}$ concentration using number of neurons and Levenberg-Marquardt training function 

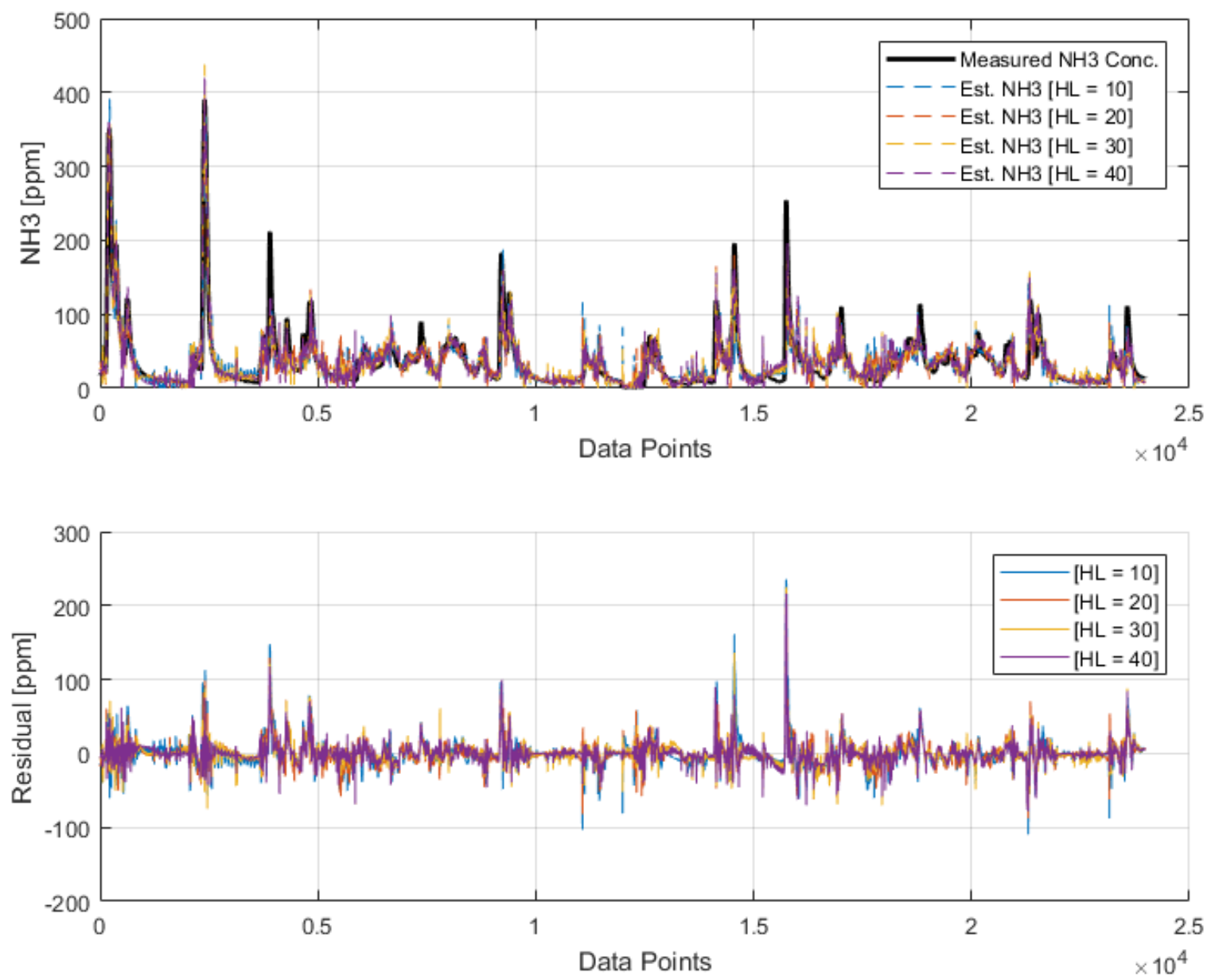

Figure 62. Comparison of measured and estimated $\mathrm{NH}_{3}$ concentration using different number of neurons and Bayesian Regularization training function

The study also evaluated performance of ANN using additional model inputs. The first derivatives of the pre-and-post TWC temperatures were calculated as using as inputs in the training a network with Bayesian Regularization and the four-same number of neurons as used earlier for the evaluation. The study uses these inputs in order to identify and estimate any additional correlation that would provide the formation of $\mathrm{NH}_{3}$ from in TWC system. Table 13 provides the performance with adding the two temperature derivatives as input to training of the model. The result indicates slight improvement in the R-squared and MSE compared to previous values. Adding the additional inputs resulted in taking more time in training the network. 
Table 13. Comparison of performance for four different number of neurons with Bayesian Regularization training function and additional two first derivative input parameters

\begin{tabular}{|r|c|c|c|c|}
\hline Number of Neurons & 10 & 20 & 30 & 40 \\
\hline R-squared & 0.858 & 0.896 & 0.919 & 0.921 \\
\hline Overall MSE & 399.1 & 276.3 & 222.7 & 221.9 \\
\hline Number of Iterations & 300 & 300 & 300 & 300 \\
\hline Training Time [sec] & 19.8 & 47.8 & 88.4 & 133.9 \\
\hline
\end{tabular}

The measured $\mathrm{NH}_{3}$ concertation were observed to be smoother, with sharp rises and slower falls. The sudden rise in $\mathrm{NH}_{3}$ peaks follows the immediate rise in power causing higher exhaust flowrate through the TWC during the cycle. On the other hand, the longer response in the $\mathrm{NH}_{3}$ drop could potentially be from the thermal and chemical inertia accompanied by the low flowrate at such conditions. The study further investigated data smoothening or filtering methods to see if certain distinct response behavior could be captured and at the same time minimize data fluctuation at lower power demand conditions. A filtering method known as exponential weighted moving average (EWMA) was evaluated for the obtained results. EWMA have been commonly used in statistical sensor estimation cases, one of its few applications in the area of on-board diagnostics for vehicle emission control systems. Unlike, other simple moving average which gives equal weighing on a period of data size provide as a time window by the user, EWMA on the other hand, applies weighting factors which decrease exponentially within the specified filtering period (FP). The filter was applied to the estimated dataset to examine if this potentially reduces the lag by applying higher weights to the estimated peaks and lower weights to the slow response of the trace. The filter performance for the best estimated data set were evaluated based on the improvement in the MSE value. Figure 63 provides the MSE results for using EWMA filter with different averaging period, and it is observed that for a period of six data points resulted in the lowest MSE value for the given data set. Figure 64, shows the comparison of the estimated $\mathrm{NH}_{3}$ concentration using between the filtered versus the unfiltered results using EWMA filtering method with period 6. Based on the residual curves, we do notice slight improvement in noise reduction. 


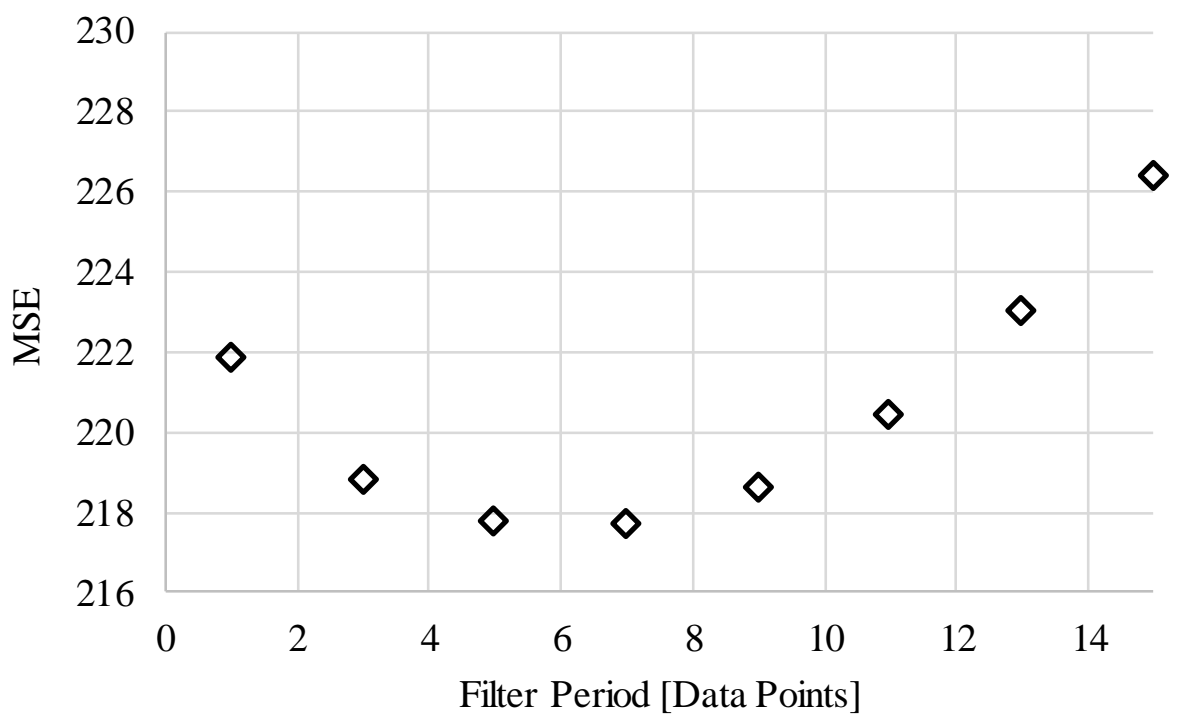

Figure 63: Comparison of MSE using EWMA filter with different filtering periods
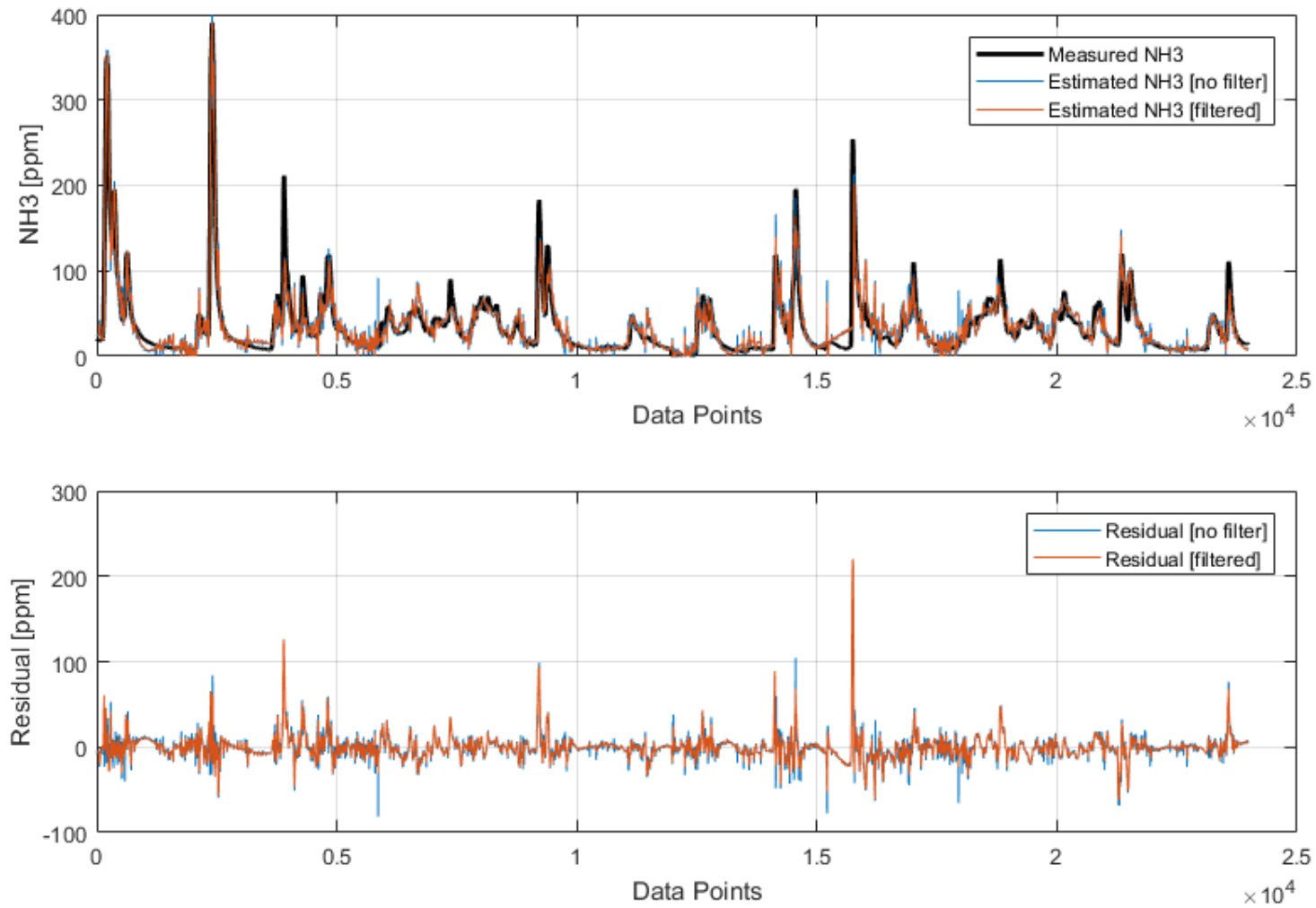

Figure 64. Comparison of measured and estimated $\mathrm{NH}_{3}$ concertation applying the EWMA filter (Filter Period = 6) 
Figure 65, shows the final estimated $\mathrm{NH}_{3}$ concentration trace using the best network properties obtained from the above parametric analysis. The following estimation resulted in having MSE equals to 218 and with a R-squared value of 0.93 . It shows that, ANN method using different engine and after-treatment parameters as in input factors can be used to capture the formation of $\mathrm{NH}_{3}$ through the catalyst with some accuracy.

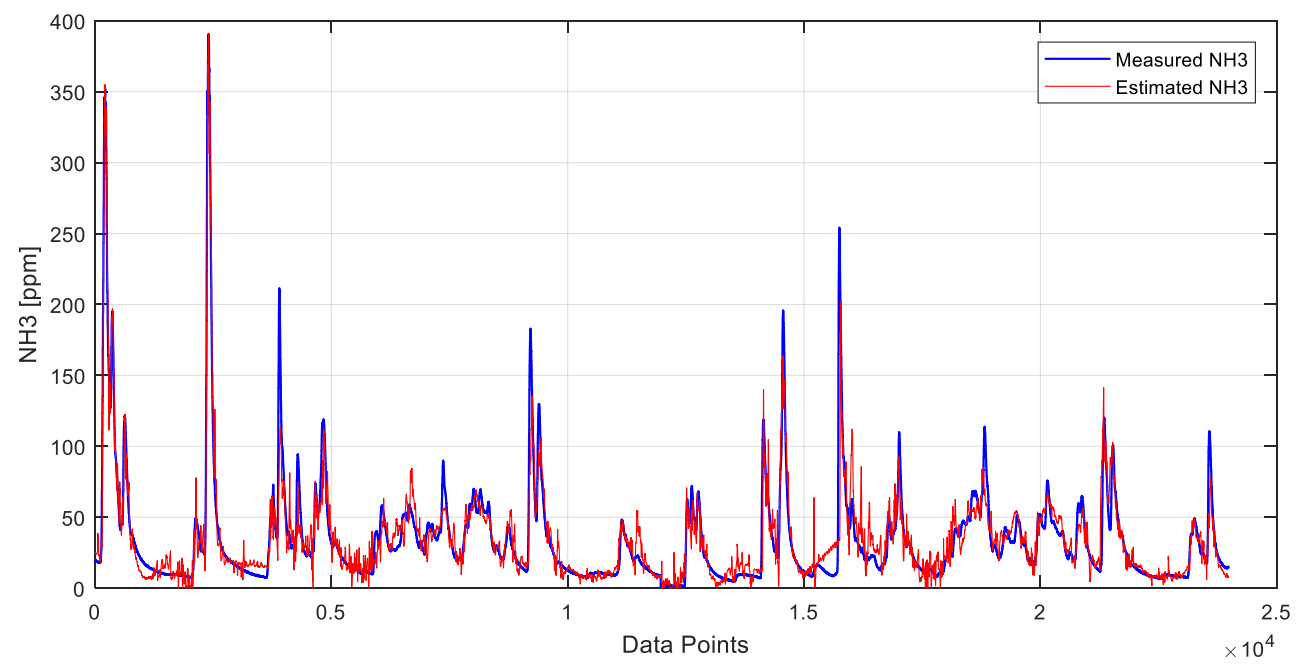

Figure 65. Comparison of measured and estimated $\mathrm{NH}_{3}$ concentration; (Number of Inputs $=9$, Number of Neurons $=40$, Fitting Function $=$ Bayesian Regularization, EWMA with Period $=6$ )

The primary objective of this specific work was to understand if precursor $\mathrm{NH}_{3}$ can be accurately predicted from the TWC using engine parameters, especially under transient conditions and then used the estimated value (instead of sensor based value) into the passiveSCR model as one of the input parameter. The dynamics of adsorption and desorption behavior is significantly influenced by the amount of $\mathrm{NH}_{3}$ entering and being stored on the surface of the SCR catalyst. As in terms of quantity, the $\mathrm{NH}_{3}$ entering the SCR can be estimated and evaluated in terms of moles per catalyst volume. The obtained estimated $\mathrm{NH}_{3}$ concertation from the ANN is converted into estimated number of moles of $\mathrm{NH}_{3}$ per SCR catalyst volume (moles/m3).

Figure 66 shows the cumulative profile of the estimated moles of $\mathrm{NH}_{3}$ per catalyst volume over the duty-cycle. This representation provides the estimation of the available $\mathrm{NH}_{3}$ that is entering the SCR and can be stored by the catalyst. From basic visual inspection, the estimated profile closely matches with the value obtained from actual measurement. The 
difference in the number of moles at a given movement into the cycle is provide by Figure 67, with a maximum value of approximately 0.28 moles $/ \mathrm{m}^{3}$ at 943 seconds into the data cycle. For the given cycle, the total moles of $\mathrm{NH}_{3}$ was calculated to be at $15.45 \mathrm{moles} / \mathrm{m}^{3}$ and 15.65 moles $/ \mathrm{m}^{3}$, using actual measurement and $\mathrm{AN}-\mathrm{N}$ estimated values, respectively.

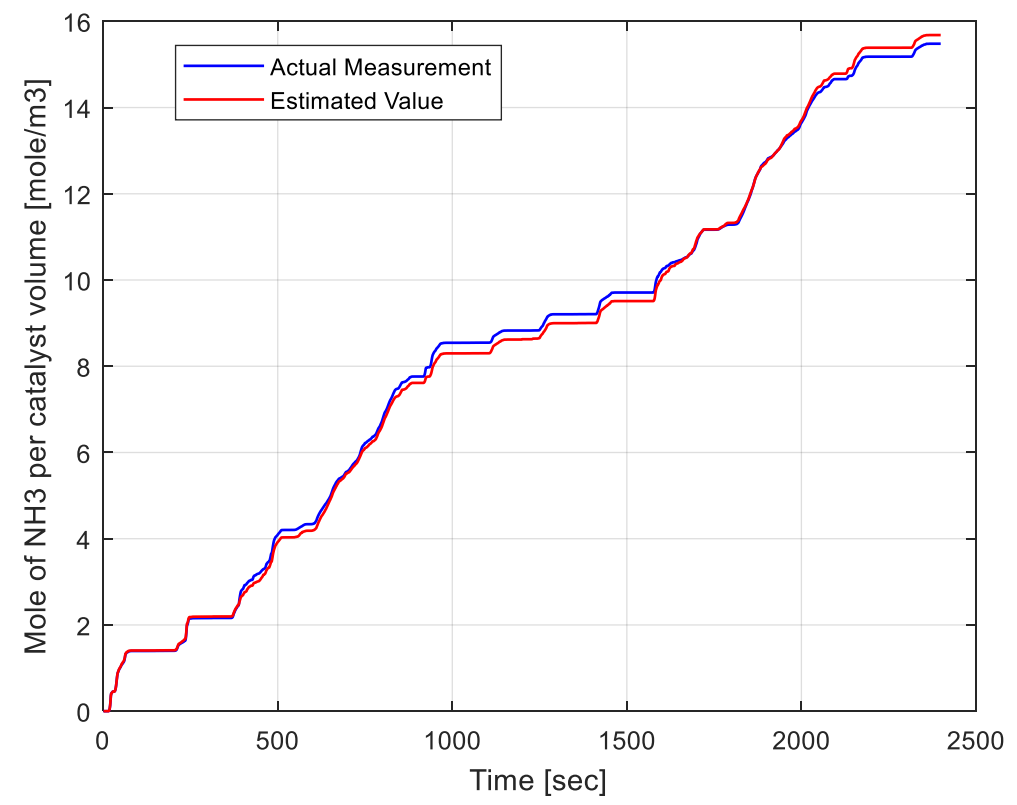

Figure 66. Comparison of number of moles of $\mathrm{NH}_{3}$ per catalyst volume $\left(\mathrm{moles} / \mathrm{m}^{3}\right)$ calculated from actual versus estimated $\mathrm{NH}_{3}$ concentration.

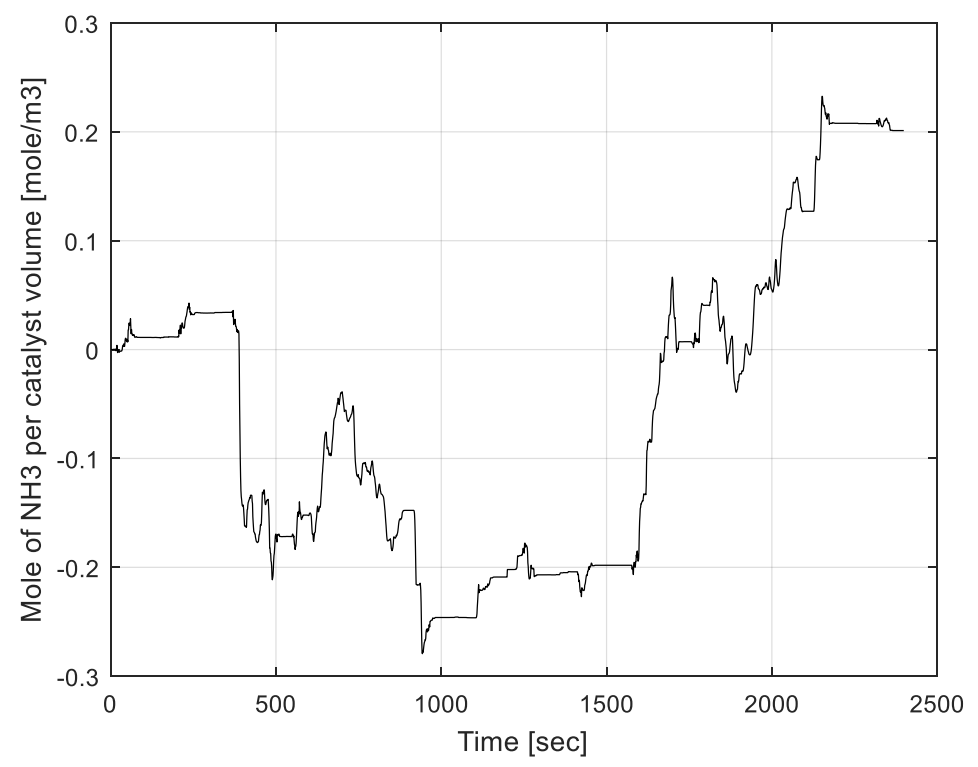

Figure 67. Difference between actual and estimated number of moles of $\mathrm{NH}_{3}$ per catalyst volume 
The best trained network obtained from the above analysis where then used to validate on a different data set. Two similar FTP tests were used for the validation process and the estimated $\mathrm{NH}_{3}$ concentration using the pre-trained network are provided by Figure 68 and Figure 70. The first data set (FTP Data Set 1) validation resulted in MSE of 330.9 and r-squared of 0.7485 , and the second set (FPT Data Set 2) resulted in MSE of 859.02 and r-squared of 0.7003. The high $\mathrm{NH}_{3}$ concentration peaks observed did vary between the two FTP cycles, and the network was able to define the higher peak but not the shorter peaks. The estimated profiles also show high data fluctuation but overall followed the trend of the measured trace (shown by the red curve). The adequacy of an AN-N trained model depend upon how well it validates a diverse set of system behavior in predicting certain response. The study does realize the shortcoming of models' limitation along with not having appropriate parameters that influence the dynamics of the TWC system in producing $\mathrm{NH}_{3}$ at various levels.

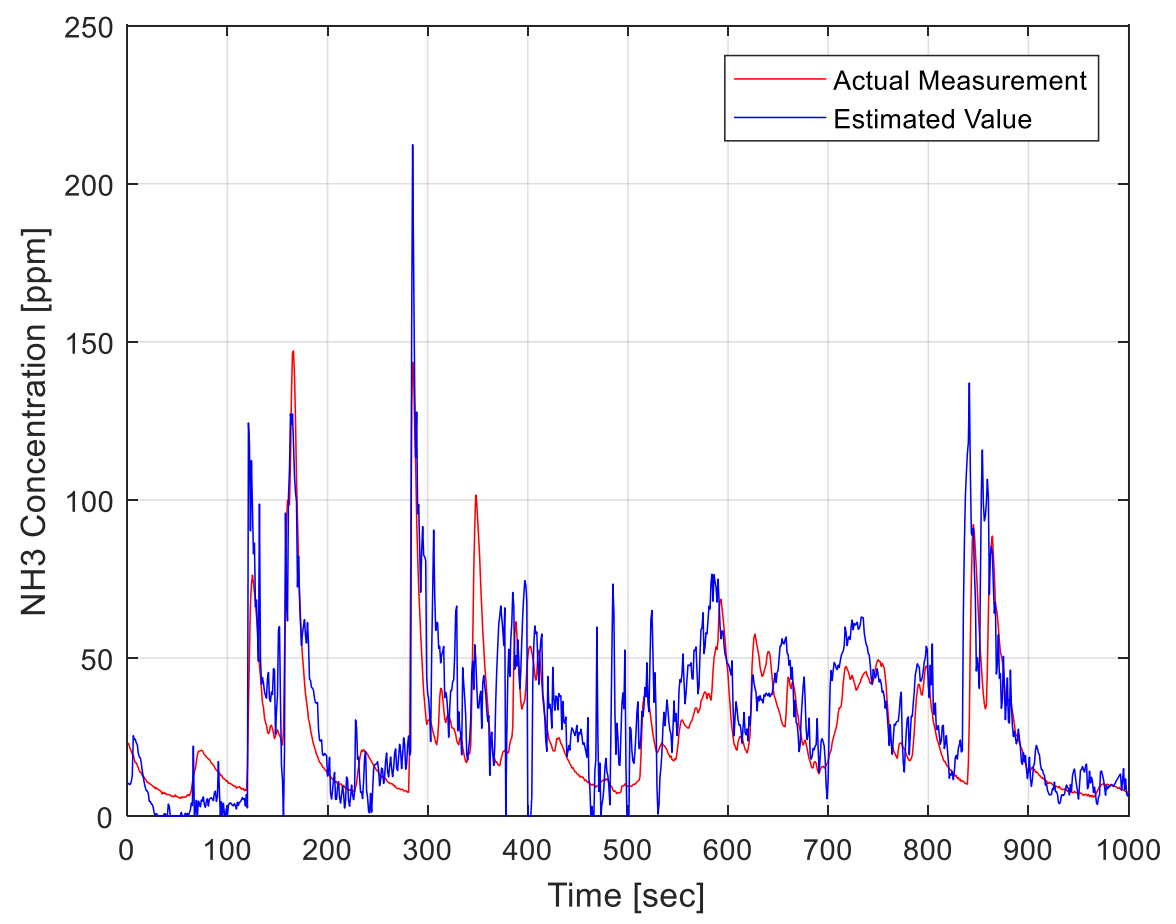

Figure 68. Comparison of actual versus estimated $\mathrm{NH}_{3}$ concertation using validation FTP Data Set 1 


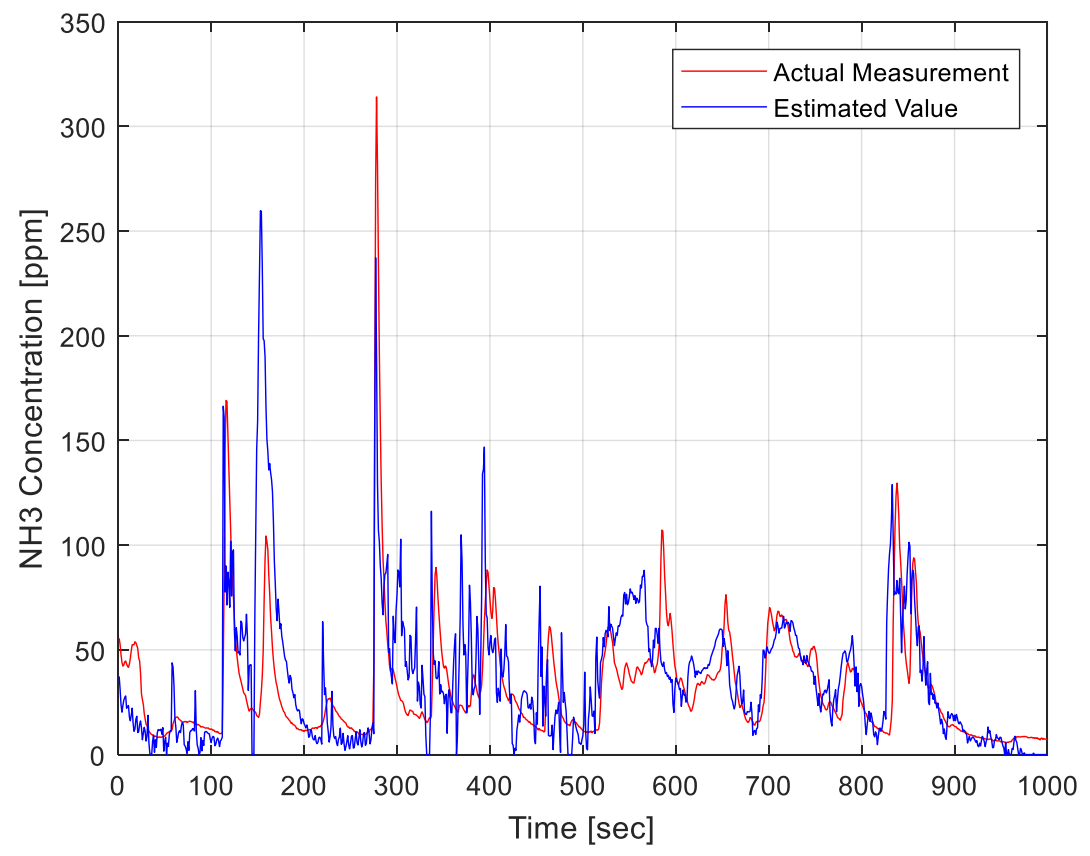

Figure 69 Comparison of actual versus estimated $\mathrm{NH}_{3}$ concertation using validation FTP Data Set 2

Figure 70 and Figure 71, represents the cumulative profile of the actual versus estimated moles of $\mathrm{NH}_{3}$ per catalyst volume over the duty-cycle. From the observation, the values using estimated concentration tend to over-predict the moles of available ammonia with a difference of $24 \%$ for Set 1 and $18.8 \%$ for the Set 2 at the end of the cycle.

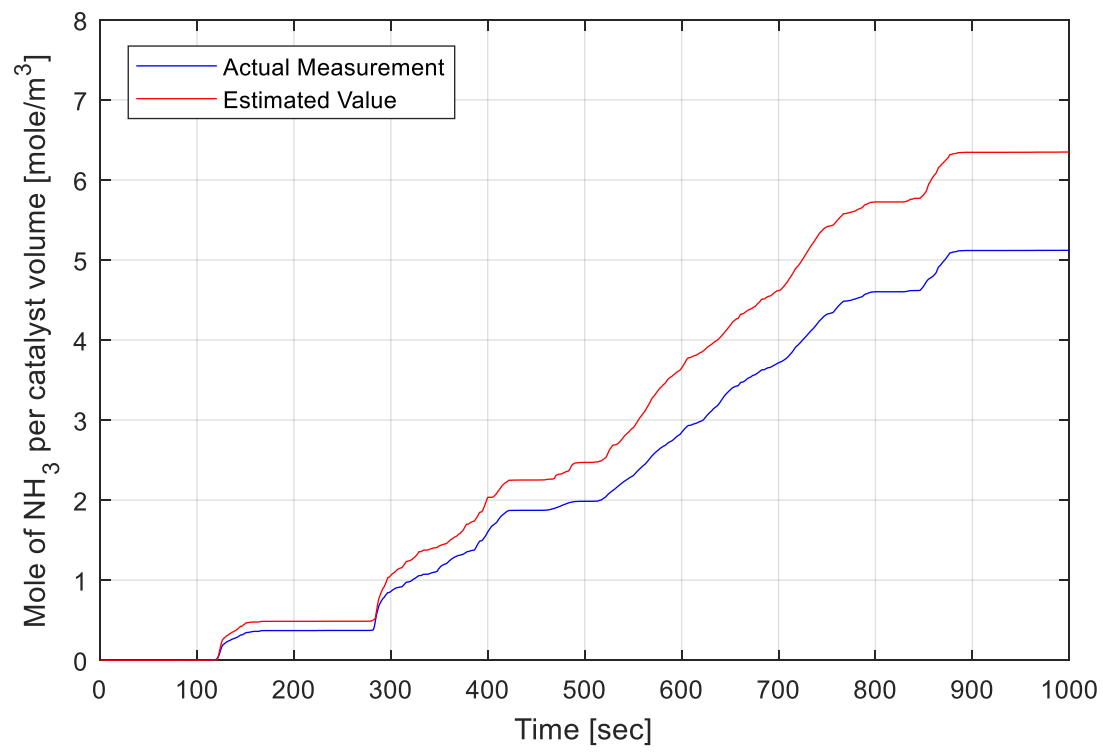

Figure 70. Comparison of actual versus estimated number of moles of $\mathrm{NH}_{3}$ per catalyst volume $\left(\mathrm{moles} / \mathrm{m}^{3}\right)$ for FTP Data Set 1 


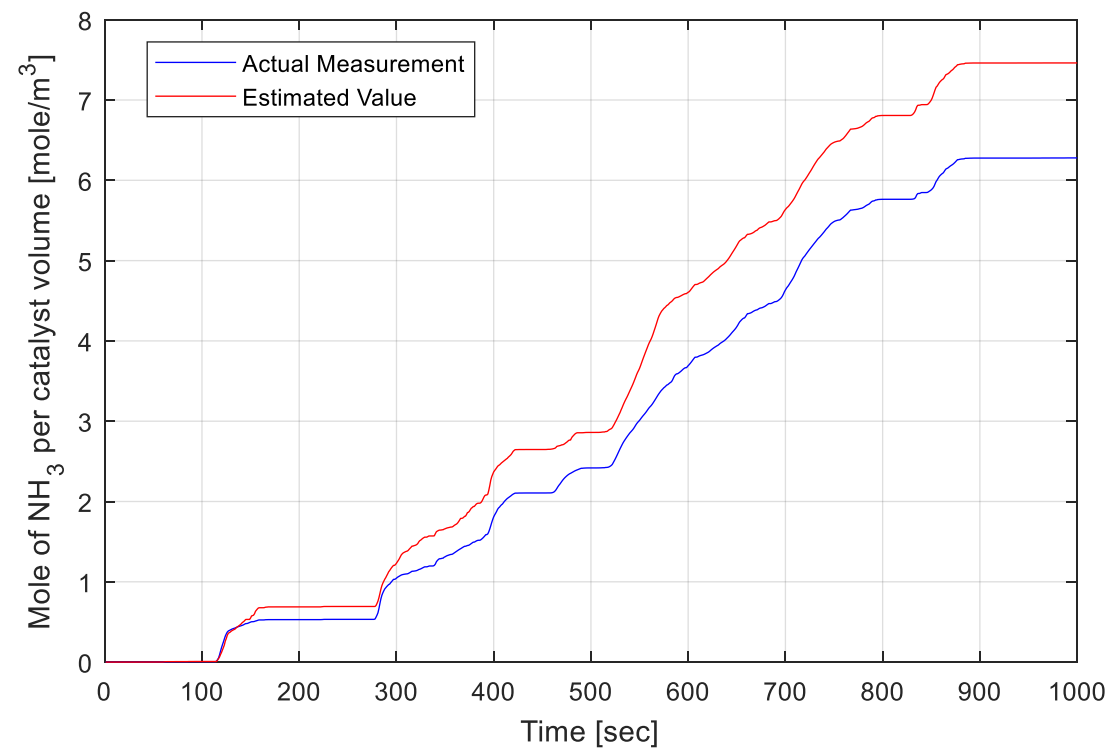

Figure 71. Comparison of actual versus estimated number of moles of $\mathrm{NH}_{3}$ per catalyst volume $\left(\mathrm{moles} / \mathrm{m}^{3}\right)$ for FTP Data Set 2

Considering the results obtained from the validation sets, the ammonia availability model can be further optimized by reducing variation in the $\mathrm{NH}_{3}$ concentration estimation. The estimated results from FTP Data Set 2 was further processed using EWMA filtering with different number of filtering periods. Although, this approach might not be practical in observing $\mathrm{NH}_{3}$ availibiltiy at a shorter resolution in the cycle but can be utilized in larger timescale observation methods.

Figure 72, shows results from applying EWMA filtering with different period numbers. There wasn't any notable change seen in $\mathrm{NH}_{3}$ availability profile when using filtering with 6 seconds and 15 seconds periods but better improvement when using a 30 second period. This can also be observed from provided Figure 73, obtained from calculating the difference between the actual and the estimated profiles. The number of moles from using 30 second settings estimation under-predicts during first half of the cycle (due to smoothening of the $\mathrm{NH}_{3}$ high peaks) and over-predicts for the other half of the cycle. This results in final estimated value of 6.91 moles $/ \mathrm{m}^{3}$, i.e. $10 \%$ higher than actual measured $6.28 \mathrm{moles} / \mathrm{m}^{3}$. 


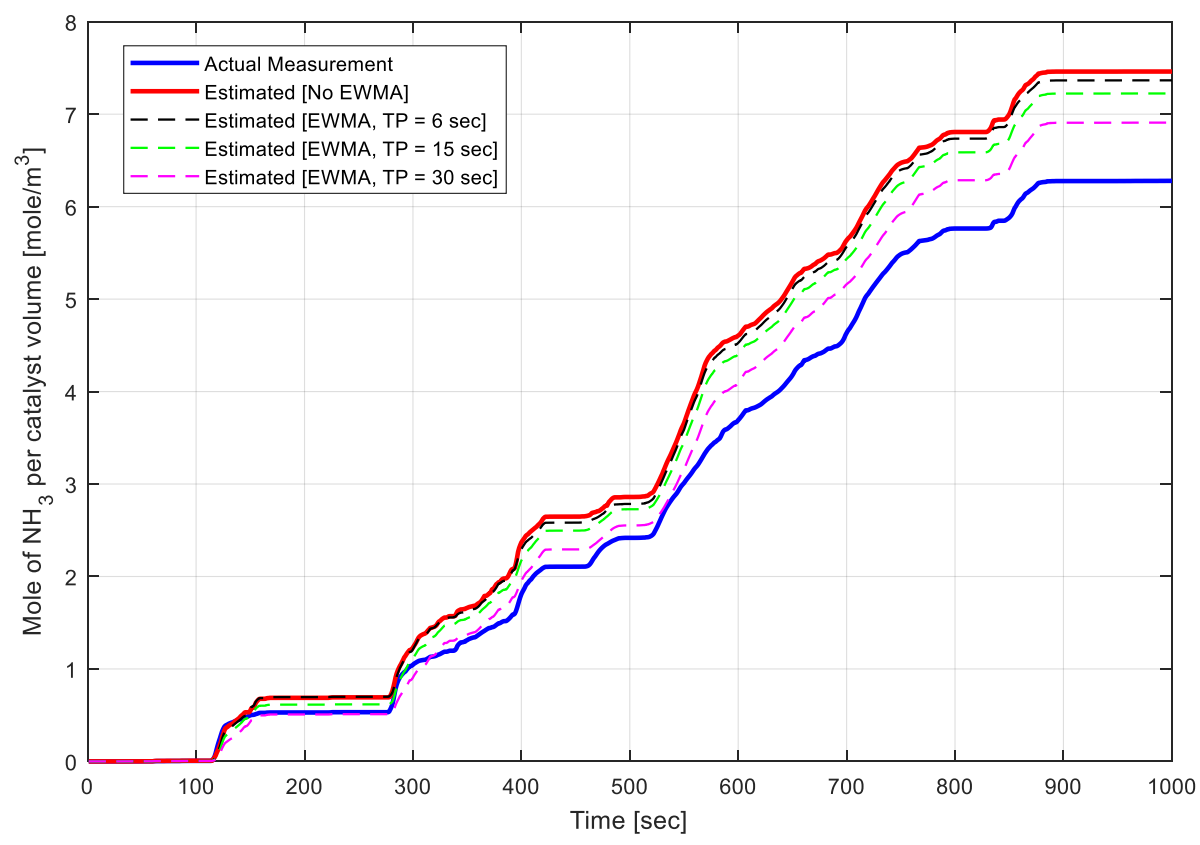

Figure 72. Comparison of actual versus estimated number of moles of $\mathrm{NH}_{3}$ per catalyst volume $\left(\right.$ moles $\left./ \mathrm{m}^{3}\right)$ periods for FTP Data Set 2 using three different EWMA filtering

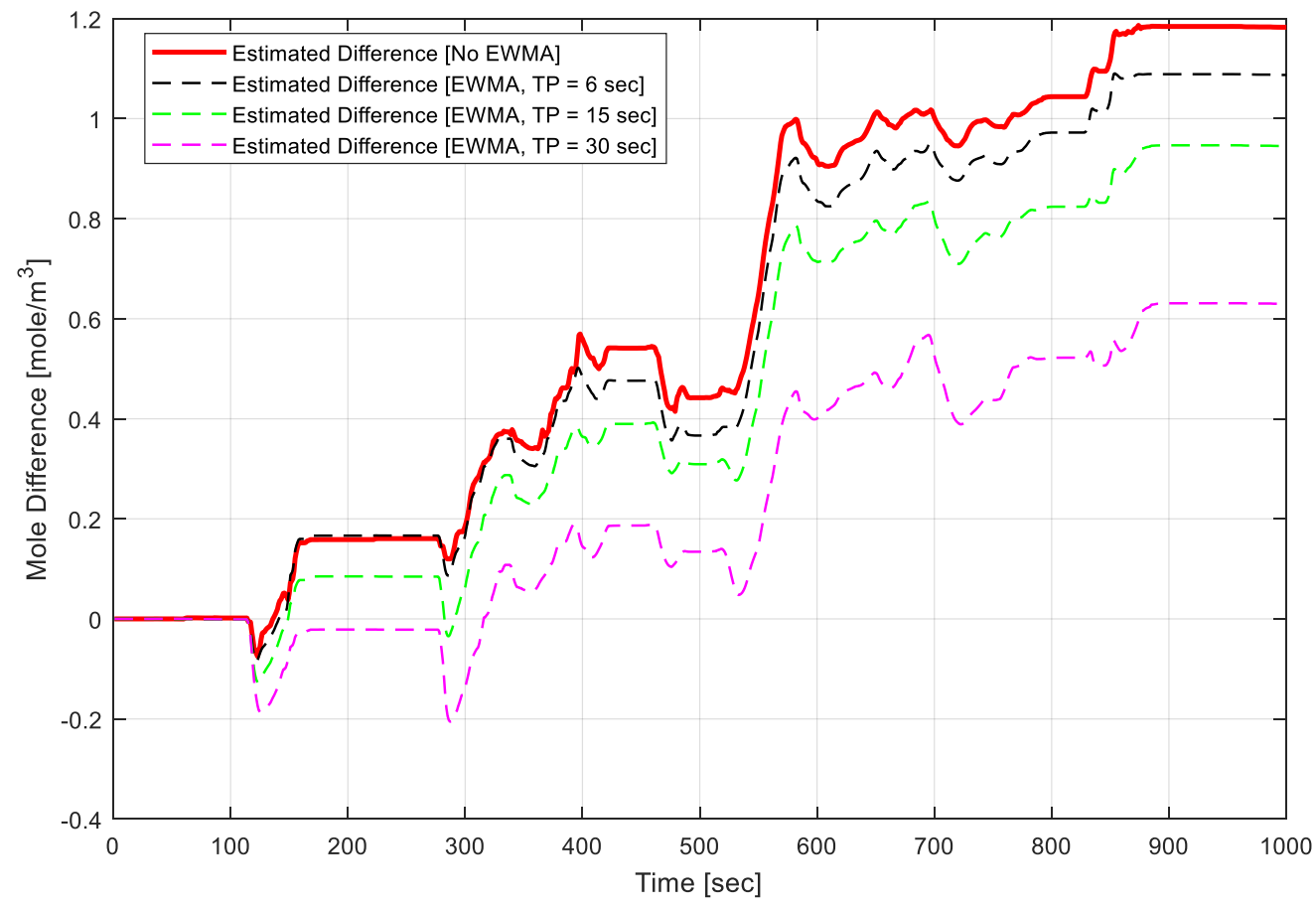

Figure 73. Difference between actual versus estimated number of moles of $\mathrm{NH}_{3}$ per catalyst volume $\left(\right.$ moles $\left./ \mathrm{m}^{3}\right)$ periods for FTP Data Set 2 using three different EWMA filtering 


\subsection{SCR model calibrated to experimental data}

Parameters of the SCR model were identified by solving the system of reaction using ODE solver and then calibrating the model against the experimental obtained data. The model calibration was aimed at matching the solved gaseous concentration to the outlet of the SCR using cost function, where the parameters are identified by minimaxing the least-square errors. The developed model results with the obtain reaction parameters was validated with the steady state mode 1 data.

Table 14 shows the reaction rate parameters identifies in the SCR state model based on the experimental data. The values obtained here closely matches with the values seen in similar literatures (Song 2013; Clark et al. 2009). The ammonia storage capacity $(\Omega)$ was assumed constant at 100 moles $/ \mathrm{m} 3$ in the calculation. The initial value for the ammonia storage ratio was assumed to be at 0.15 .

Table 14. Reaction rate identified parameters in the three state SCR model

\begin{tabular}{|c|c|c|c|}
\hline $\begin{array}{c}\text { Parameter } \\
\text { Number }\end{array}$ & Parameter & $\begin{array}{c}\text { Optimized } \\
\text { Values }\end{array}$ & Unit \\
\hline 1 & $\mathrm{k}_{\text {reduction }}$ & $3.63 \mathrm{E}+06$ & $\mathrm{~m} 3 / \mathrm{mol}-\mathrm{s}$ \\
\hline 2 & $\mathrm{E}_{\text {reduction }}$ & 65.2 & $\mathrm{~kJ} / \mathrm{mol}$ \\
\hline 3 & $\mathrm{k}_{\text {adsorption }}$ & $5.48 \mathrm{E}+06$ & $\mathrm{~m} 3 / \mathrm{mol}-\mathrm{s}$ \\
\hline 4 & $\mathrm{E}_{\text {adsorption }}$ & 47.6 & $\mathrm{~kJ} / \mathrm{mol}$ \\
\hline 5 & $\mathrm{k}_{\text {desorption }}$ & $3.36 \mathrm{E}+06$ & $1 / 2 \mathrm{~s}$ \\
\hline 6 & $\mathrm{E}_{\text {desorption }}$ & 131 & $\mathrm{~kJ} / \mathrm{mol}$ \\
\hline
\end{tabular}

Figure 74 displays comparison of model predicted NOx values with the measured concentration using FTIR pre-and-post SCR. It can be seen the simulated NOx after the SCR matches well before the $40^{\text {th }}$ second but then overpredicts for the rest of the part. The initial value of the ammonia storage ratio plays a key role due to the difference of the initial guess of the ammonia storage for the model and the true storage ratio inside the SCR catalyst. This can also be observed from the simulated ammonia storage ratio curve (shown by the dashed green line in the figure) where ammonia storage ratio rapidly drops to zero indicating no $\mathrm{NH}_{3}$ stored for NOx reduction reaction. Additionally, the reaction rates of the SCR reactions are highly dependent on the $\mathrm{NH}_{3}$ storage capacity and changed the model outcome since the value participates in all three state reactions. The simulation was rerun for a higher initial ammonia storage value (0.7) using the same reaction parameters and comparison is shown inn Figure 
75. The result indicates higher ammonia storage ratio till the 250 second with closer NOx prediction than compared to the previous results.
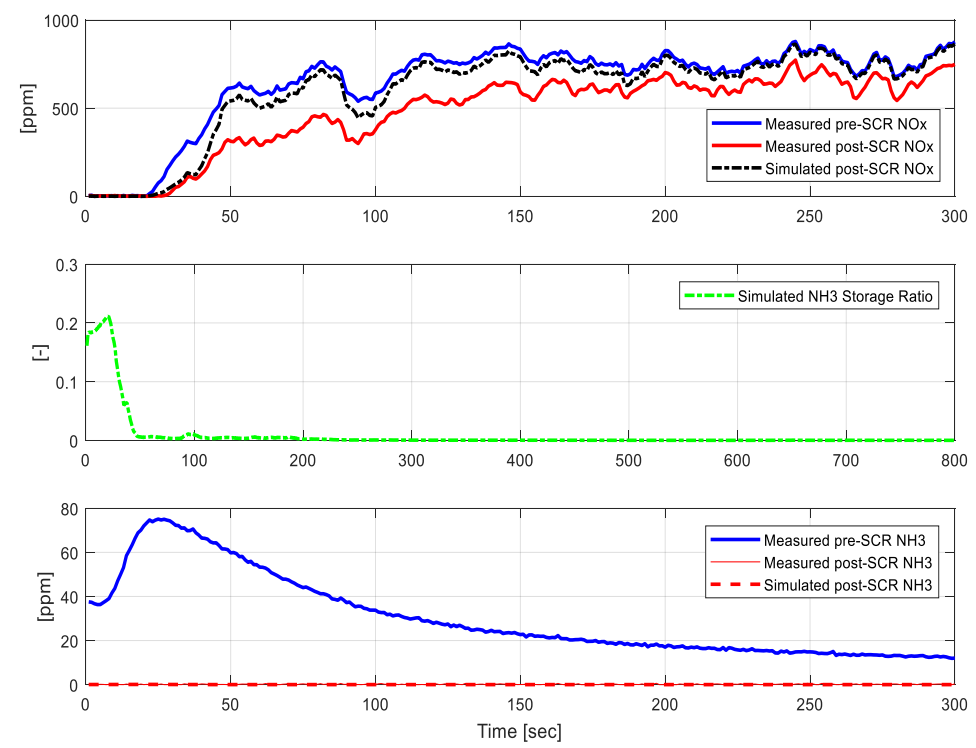

Figure 74. Comparison of post-SCR NOx, post-SCR $\mathrm{NH}_{3}$ and $\mathrm{NH}_{3}$ storage ratio predicted by 3-state SCR model storage ratio for steady-state Mode 1 (baseline operation); Initial ammonia storage ratio $=0.15$
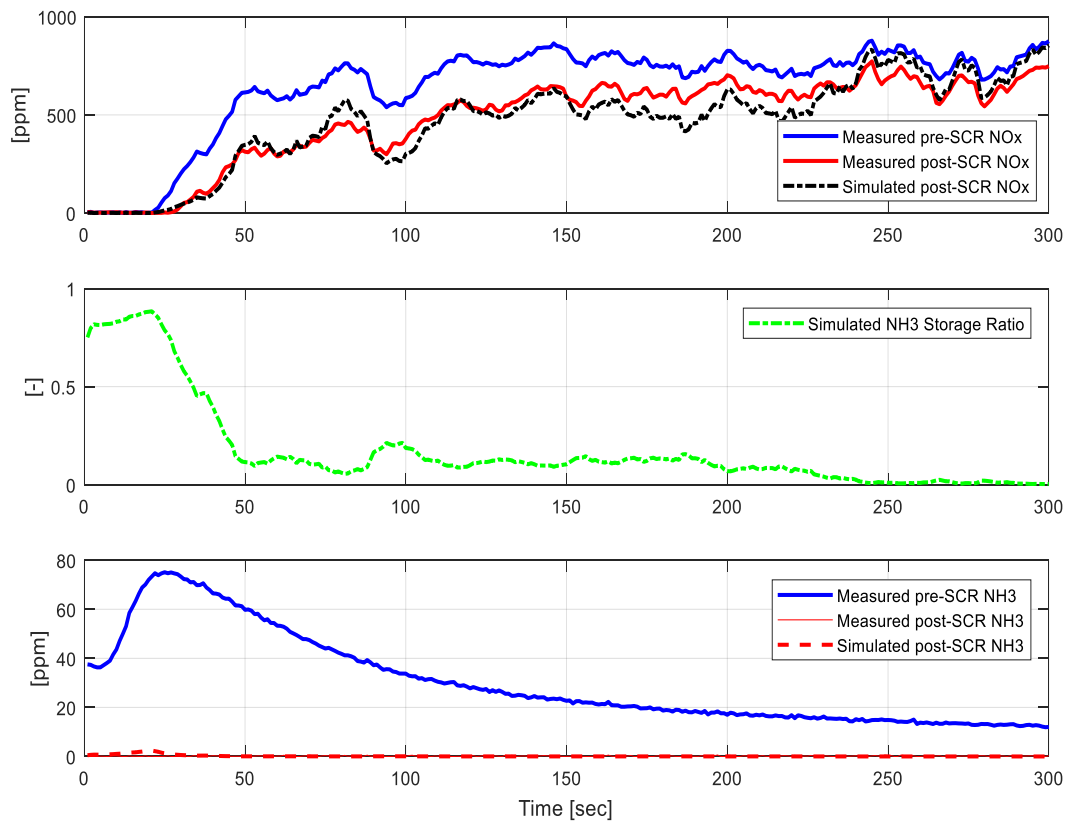

Figure 75. Comparison of post-SCR NOx, post-SCR $\mathrm{NH}_{3}$ and $\mathrm{NH}_{3}$ storage ratio predicted by 3-state SCR model for steady-state Mode 1 (baseline operation); Initial ammonia storage ratio $=0.7$; 
Figure 76 provides results simulated from considering the $\mathrm{NH}_{3}$ oxidation mechanism and adding three more parameters $(\mathrm{E}$ and $\mathrm{k}$ for oxidation, and $\Omega$ ) as in the identification process. The initial $\mathrm{NH}_{3}$ storage ratio was set at 0.15 for this simulation. The comparison of the simulated NOx profile and the measured show good agreement till $150^{\text {th }}$ second and then tend to underestimate into rest of the cycle duration, except between (305-420) seconds. And, these disparities could be due to $\mathrm{NO}_{2}$ formation that was seen in the actual measurement. The study did not include $\mathrm{NO}_{2}$ formation mechanism in the SCR model.
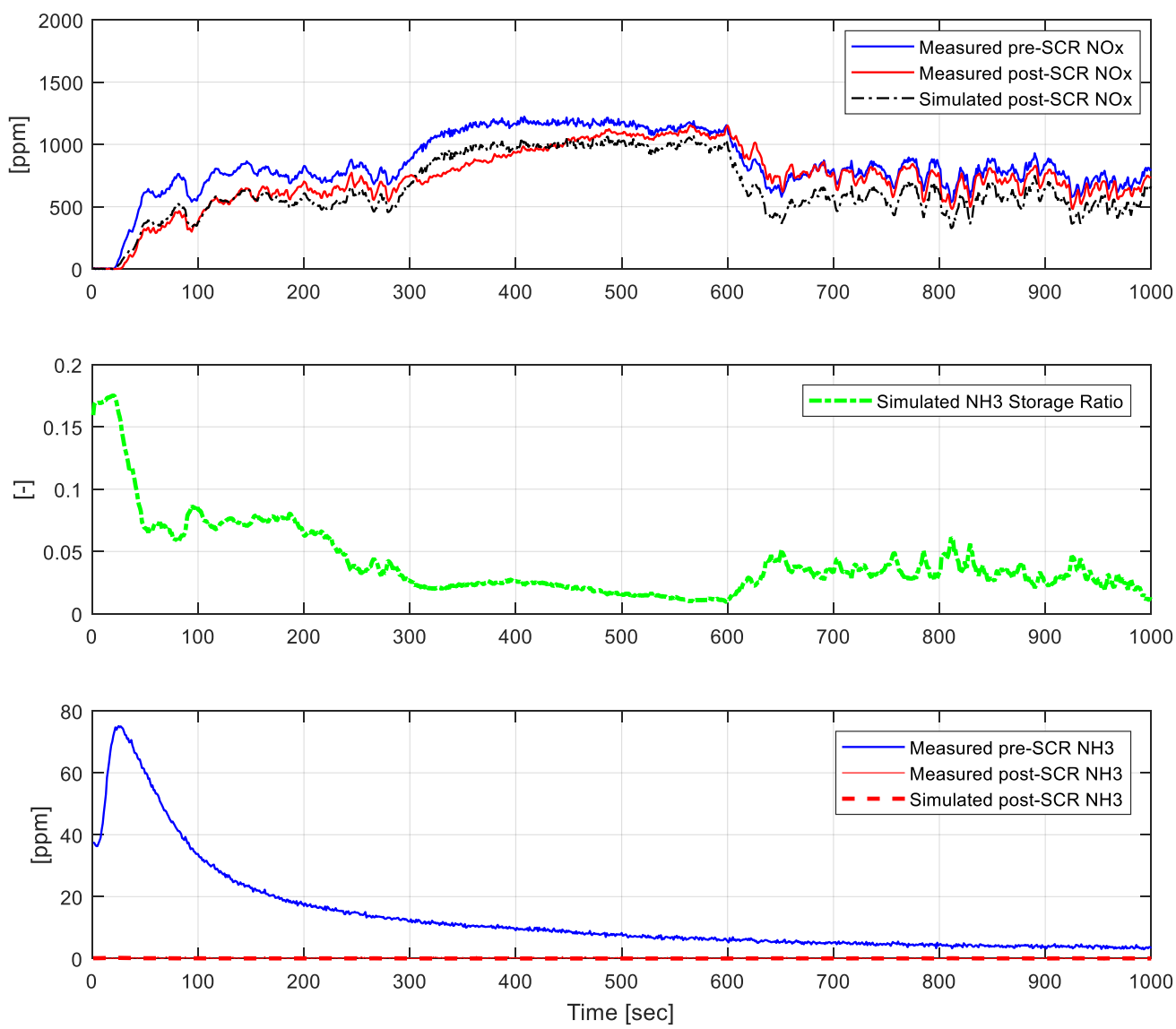

Figure 76. Comparison of post-SCR NOx, post-SCR $\mathrm{NH}_{3}$ and $\mathrm{NH}_{3}$ storage ratio predicted by 3-state SCR model for steady-state Mode 1 (baseline operation); Initial ammonia storage ratio $=0.15 ; \Omega$ as identified parameter

Table 15 summarizes the identified values of 9 model parameters using GA's optimization tool. Ones again the obtained parameter correlates well with the ones provided in literatures. 
Table 15. Reaction rate identified parameters in the three state SCR model

\begin{tabular}{|c|l|c|r|}
\hline $\begin{array}{c}\text { Parameter } \\
\text { Number }\end{array}$ & \multicolumn{1}{|c|}{ Parameter } & Optimized Values & \multicolumn{1}{c|}{ Units } \\
\hline 1 & $\mathrm{k}_{\text {reduction }}$ & $2.84 \mathrm{E}+05$ & $\mathrm{~m}^{3} / \mathrm{mol}-\mathrm{s}$ \\
\hline 2 & $\mathrm{E}_{\text {reduction }}$ & 70.92 & $\mathrm{~kJ} / \mathrm{mol}$ \\
\hline 3 & $\mathrm{k}_{\text {adsorption }}$ & $5.45 \mathrm{E}+06$ & $\mathrm{~m} 3 / \mathrm{mol}-\mathrm{s}$ \\
\hline 4 & $\mathrm{E}_{\text {adsorption }}$ & 46.6 & $\mathrm{~kJ} / \mathrm{mol}$ \\
\hline 5 & $\mathrm{k}_{\text {desorption }}$ & $3.33 \mathrm{E}+09$ & $1 / \mathrm{s}$ \\
\hline 6 & $E_{\text {desorption }}$ & 125.1 & $\mathrm{~kJ} / \mathrm{mol}$ \\
\hline 7 & $\mathrm{k}_{\text {oxidation }}$ & $6.57 \mathrm{E}+07$ & $1 / \mathrm{s}$ \\
\hline 8 & $E_{\text {oxidation }}$ & 129.8 & $\mathrm{~kJ} / \mathrm{mol}$ \\
\hline 9 & $\Omega$ & 76.63 & $\mathrm{moles} / \mathrm{m}^{3}$ \\
\hline
\end{tabular}




\section{CHAPTER 6 Conclusions}

The study presents evaluation of a pathway in mitigating secondary $\mathrm{NH}_{3}$ emission produced by the TWC during stoichiometric operation of natural gas engines. The study approaches the development of a passive ammonia reduction system by conducting experiments on a heavy-duty natural gas engine and utilizing selective catalytic reduction units typically used in diesel engines. The MY08 ISL-G Cummins engine equipped with the TWC after-treatment system was evaluated for baseline performance and emission characteristics and tested over transient and steady-state operating conditions. The study further evaluates an engine-based control strategy to minimize both $\mathrm{NH}_{3}$ and NOx control over the TWC+SCR configuration. The following sections highlights the major findings, provides recommendations, and contextualizes studies contribution to relevant areas of interest.

\subsection{Experimental Findings}

The results obtained from the baseline evaluation indicated elevated levels of tailpipe $\mathrm{NH}_{3}$ emission, with an average concentration of $37 \mathrm{ppm}$ and $61 \mathrm{ppm}$ for the FTP and WHTC transient cycles, respectively. The high peaks of $\mathrm{NH}_{3}$ observed were caused by isolated rich AFR conditions due to aggressive power demands within the tested cycles. Additionally, exhaust temperatures were a crucial factor in $\mathrm{NOx}$ reduction and $\mathrm{NH}_{3}$ formation, where improved $\mathrm{NOx}$ conversions were observed at higher TWC inlet temperatures but also resulted in increased $\mathrm{NH}_{3}$ formation over the TWC.

Correspondingly, the results obtained from evaluating five different formulated SCRs showed that SCR 3 (Fe-zeolite with $400 \mathrm{cpsi}$ ) and SCR 4 (Cu-zeolite) absorbed above 90\% of the TWC's generated $\mathrm{NH}_{3}$ below $375^{\circ} \mathrm{C}$ of averaged SCR temperatures. However, the Fe-zeolite performed with 60-85\% efficiency in $\mathrm{NO}_{\mathrm{x}}$ conversion above $300^{\circ} \mathrm{C}$, while the Cu-zeolite performed moderately between (40-65\%) at all temperature ranges. Performance-based on NOx conversion efficiency and storage capacity at both low and higher temperature ranges, observed under dynamic natural gas engine operations, the study considered the Cu-zeolite SCR for an adsorption and desorption study over eight steady-state modes. From these steady-state mode experiments, it was observed that $\mathrm{NH}_{3}$ storage ratio and SCR catalyst temperature were the two main factors in initiating desorption. Furthermore, from switching the engine to lean-burn operation, the high NOx activity after the TWC could regenerate the stored $\mathrm{NH}_{3}$, however, the 
levels in $\mathrm{NH}_{3}$ regeneration and $\mathrm{NOx}$ conversion depended upon different lean-ON duration. The study compared different lean-ON periods evaluated for a medium speed/load operation mode (Mode 6). Lean-ON duration between (2.5-3.5) seconds resulted in low moderate NOx slip after the TWC, improved NOx conversion by the SCR and initiated $\mathrm{NH}_{3}$ regeneration process. The study also evaluated fuel consumption differences, and the results indicated on average a $2.1 \%$ brake specific fuel consumption improvement for the lean-ON operation when compared to baseline stoichiometric operation over the entire 8 steady-state modes.

The study further investigated model-based estimator methods from a stand-point of control-oriented passive-SCR regeneration approach. Results obtained from Model 1 indicated that the ANN fitting method estimated accumulated pre-SCR $\mathrm{NH}_{3}$ in moles per SCR volume to be $1.3 \%$ higher than the actual measured when using 9 different inputs form two combined FTP data sets for training. However, during the validation process, the ANN estimated significantly higher differences of $25.6 \%$ and $16.8 \%$ for two different individual FTP data sets. The study further evaluated using an EWMA filter with different filtering windows, and showed that the accumulation of $\mathrm{NH}_{3}$ at the SCR inlet could be estimated more accurately at a larger time scale.

On the other hand, results obtained from Model 2 adequately estimated post-SCR NOx and $\mathrm{NH}_{3}$ adsorption for lower speed/load steady-state modes but the model was not able to estimate $\mathrm{NH}_{3}$ desorption rise at higher steady-state modes. The differences observed in postSCR NOx and $\mathrm{NH}_{3}$ could be attributed to assuming an initial $\mathrm{NH}_{3}$ storage ratio that could have been different from the actual $\mathrm{NH}_{3}$ storage of the SCR in the beginning of each steady-state test. Additionally, the ammonia storage capacity, which have shown to vary with SCR surface temperature from other related studies, was kept constant during the parameter identification process.

\subsection{Recommendations}

The current study was limited by the SCR design parameters, and the performance of the SCR in $\mathrm{NH}_{3}$ storage and NOx reduction characteristics could be improved with specifically designed catalyst to meet the natural gas engine operation's ranges. In addition, the study observed $\mathrm{NO}_{2}$ being formed over the Cu-zeolite SCR catalyst with $\mathrm{NO}_{2} / \mathrm{NO}$ ratios as close to 1:1 under certain conditions. As a result, a two stage SCR system could potentially allow improvements that considers the fast-SCR reactions. 
Beside from the adequate SCR system downstream of the TWC, the engine based control strategy that was developed using fuzzy logic method, can be improved in an experimental study using a H-I-L setup to fine-tune possible input-output parameters. For example, the size of membership functions and the inference rule matrix could be increased for better regeneration status control. Along with the size, other shapes in defining the membership functions could also be considered.

Ammonia storage ratio has shown to be a good indicator in estimating the state of the SCR, and utilizing this as one of the fuzzy input parameters would allow wider range of SCR regeneration control. For example, the regen status could be switched to ON mode by monitoring the ammonia storage ratio which indicates saturation level of the SCR catalyst over a duty cycle, even when there is no $\mathrm{NH}_{3}$ or $\mathrm{NOx}$ being slipped after the SCR. This would allow improved fuel consumptions due to more frequent lean-ON operations.

The study was limited with engine and after-treatment parameters that were used in the ANN Model-1. However, the estimation could be improved by conducting further analysis with other influential factors that links to formation of $\mathrm{NH}_{3}$ over the TWC. For example, using engine parameters such as actual measured air fuel ratio, mass rate of EGR, in-cylinder combustion parameters, and more. Using additional emission sensors at the inlet of the TWC and instrumenting mid-brick temperatures would potentially help in improving the estimation accuracy but could lead to adding hardware cost and increasing computational time.

On the other hand, Model-2 could also be improved by assuming more higher order reaction processes and adding potential SCR mechanisms that were not considered in this study. Although, the study used only the inlet SCR temperatures, using catalyst temperature models through the catalyst could potentially help in determining desorption characteristics as observed during the transient and steady-state tests.

\subsection{Contributions}

This dissertation contributes to the area of alternative fueled vehicles and their emission reduction challenges. Few researchers have addressed the concerns over high emission levels of ammonia observed from stoichiometric operated natural gas vehicles equipped with a TWC system, and previous work primarily focused on gasoline fueled engines only. As a preliminary part of this work, the study investigated a heavy-duty natural gas engine with a comprehensive evaluation of ammonia formation over the three-way catalyst in relation to dynamic engine 
operating parameters. The results obtained in this study pose a critical need to monitor $\mathrm{NH}_{3}$ emission from stoichiometric natural gas vehicles which in the near future could be regulated at tailpipe levels as seen in diesel vehicles with urea-SCR technology.

In addition, the passive ammonia reduction pathway utilizing an SCR system that was evaluated in this study could also lead to meeting the low-NOx emission standards. The USEPA has initiated rulemaking for low-NOx emission standards, and if in force, the new standard would require all new heavy-duty vehicles to meet $0.02 \mathrm{~g} / \mathrm{bhp}-\mathrm{hr} \mathrm{NOx}$ regulation starting from the year 2024. This would involve vehicle manufacturers to reduce NOx emission by almost $90 \%$ from the current standards, and would require significant after-treatment development strategies.

As a final remark, this study specifically introduces techniques in operating dedicated natural gas engines at or near stoichiometric point to more lean-burn combustion regimes. Engines deigned for more lean-burn operation can make use of higher compression ratios, and thus provide improved performance and more efficient fuel use than those in conventional spark ignited engines. The engine-based air-fuel ratio dithering strategy using a fuzzy-logic controller was designed to lower both $\mathrm{NH}_{3}$ and $\mathrm{NOx}$ emissions, however, has also shown to provide fuel consumption improvements. The fuzzy-logic controller which needs only two sensor values as inputs can further be expanded into using ammonia storage ratios as one of the linguistic variables. In addition, with the two-regeneration outputs, short-dithering and longdithering modes, would further expand the operation of lean-burn activity to a much larger scale. This would be a promising pathway for off-road and stationary dedicated natural gas engines since the air-fuel ratio could be precisely controlled over steady-state duty cycles, also from a model-based ammonia storage and control development standpoint. 


\section{REFERENCES}

Barbier, Jacques, and Daniel Duprez. 1994. "Steam Effects in Three-Way Catalysis." Applied Catalysis B: Environmental 4 (2): 105-40. doi:http://dx.doi.org/10.1016/0926-3373(94)80046-4.

Battye Viney P., Roelle, Paul A., William; Aneja. 2003. "Evaluation and Improvement of Ammonia Emissions Inventories." Atmospheric Environment 37 (2003): 3873-83.

Blanco-Rodriguez, Dr-Ing David. 2014. Modelling and Observation of Exhaust Gas Concentrations for Diesel Engine Control. doi:10.1007/978-3-319-06737-7.

Bosch, Hans, and Frans Janssen. 1988. "Catalytic Reduction of Nitrogen Oxides.” Catalysis Today 2 (4): V.

Cavataio, Giovanni, Hung-wen Jen, James R Warner, James W Girard, Jeong Y Kim, and Christine K Lambert. 2008. "Enhanced Durability of a Cu / Zeolite Based SCR Catalyst." SAE International Journal of Fuels and Lubricants 1 (1): 477-87. doi:10.4271/2008-01-1025.

Cesare, Matteo De, and Federico Covassin. 2011. "Neural Network Based Models for Virtual NOx Sensing of Compression Ignition Engines.” SAE International. doi:10.4271/2011-24-0157.

CFR40/1065/SubB. 2017. "\$1065.140 Dilution for Gaseous and PM Constituents." Accessed November 21. https://www.ecfr.gov/.

CFR40/1065/SubD. 2017. "Subpart D - Calibrations and Verifications." Accessed November 21. https://www.ecfr.gov/.

Chatterjee, Daniel, Thomas Burkhardt, Brigitte Bandl-Konrad, Tillmann Braun, Enrico Tronconi, Isabella Nova, and Cristian Ciardelli. 2005. "Numerical Simulation of Ammonia SCR-Catalytic Converters : Model Development and Application." SAE Transactions 114 (4): 437-48. doi:10.4271/2005-01-0965.

Chavannavar, Praveen. 2014. "Development and Implementation of a Mapless, Model Based SCR Control System.” SAE International. doi:10.4271/2014-01-9050.

Chen, Pingen, and Junmin Wang. 2014. "Sliding-Mode Observers for Urea Selective Catalytic Reduction System State Estimations Based on Nitrogen Oxide Sensor Measurements." Proceedings of the Institution of Mechanical Engineers, Part D: Journal of Automobile Engineering 229 (7): 954407014548052. doi:10.1177/0954407014548052.

Chen, Pingen, and Junmin Wang. 2015. "A Novel Cost-Effective Robust Approach for Selective Catalytic Reduction State Estimations Using Dual Nitrogen Oxide Sensors." Proceedings of the Institution of Mechanical Engineers, Part D: Journal of Automobile Engineering 229 (1): 83-96. doi:10.1177/0954407014526570.

Chi, John N. 2009. "Control Challenges for Optimal NOx Conversion Efficiency from SCR Aftertreatment Systems.” SAE International. doi:10.4271/2009-01-0905.

Ciardelli, Cristian, Isabella Nova, Enrico Tronconi, Brigitte Konrad, Daniel Chatterjee, Karlheinz Ecke, and Michel Weibel. 2004. "SCR-DeNOx for Diesel Engine Exhaust Aftertreatment: UnsteadyState Kinetic Study and Monolith Reactor Modelling." Chemical Engineering Science 59 (22-23): 5301-9. doi:10.1016/j.ces.2004.07.016.

Clark, Nigel, D Ph, Gregory Thompson, D Ph, Mridul Gautam, D Ph, Benjamin Shade, et al. 2009. "Optimization of a Retrofit Urea-SCR System Clinton R . Bedick Dissertation Submitted to the College of Engineering and Mineral Resources at West Virginia University in Partial Fulfillment of 
the Requirements for the Degree of Doctor of Philosophy in Mecha."

Colombo, Massimo, Grigorios Koltsakis, Isabella Nova, and Enrico Tronconi. 2012. "Modelling the Ammonia Adsorption-desorption Process over an Fe-zeolite Catalyst for SCR Automotive Applications." Catalysis Today 188 (1): 42-52. doi:http://dx.doi.org/10.1016/j.cattod.2011.09.002.

Colombo, Massimo, Isabella Nova, and Enrico Tronconi. 2010. "A Comparative Study of the NH3SCR Reactions over a Cu-Zeolite and a Fe-Zeolite Catalyst." Catalysis Today 151 (3-4): 223-30. doi:http://dx.doi.org/10.1016/j.cattod.2010.01.010.

Defoort, M, D Olsen, and B Willson. 2004. "The Effect of Air-Fuel Ratio Control Strategies on Nitrogen Compound Formation in Three-Way Catalysts." International Journal of Engine Research 5 (1): 115-22. doi:10.1243/146808704772914291.

Delgado, Oscar, and Rachel Muncrief. 2015. “Assessment of Heavy-Duty Natural Gas Vehicle Emissions: Implications and Policy Recommendations." http://www.theicct.org/sites/default/files/publications/ICCT_NG-HDV-emissionsassessmnt_20150730.pdf.

Devarakonda, M, J Johnson, and G Parker. 2012. "NOx Control Systems and Methods for Controlling NOx Emissions" US20120260.

Devarakonda, M, G Parker, and J H Johnson. 2008. "Adequacy of Reduced Order Models for ModelBased Control in a Urea-SCR Aftertreatment System.” SAE Technical Paper Series 2008 (724): 776-90. doi:10.4271/2008-01-0617.

Eijnden, Edwin v d, Robert Cloudt, Frank Willems, and Peter v d Heijden. 2009. "Automated Model Fit Tool for SCR Control and OBD Development.” SAE International. doi:10.4271/2009-01-1285.

Figura, Jiri, Dejan Kihas, Jaroslav Pekar, Michael Uchanski, Nassim Khaled, and Sriram Srinivasan. 2016. "Automotive Selective Catalytic Reduction System Model-Based Estimators for On-ECU Implementation: A Brief Overview.” SAE International. doi:10.4271/2016-01-0972.

Franco S. Francisco; German, John; Mock, Peter, Vicente; Posada. 2014. "Real-World Exhaust Emissions from Modern Diesel Cars Part 1: Aggregated Results.” The International Council on Clean Transportation.

Frobert, Arnaud, Yann Creff, Stéphane Raux, Christophe Charial, Arnaud Audouin, and Laurent Gagnepain. 2009. "SCR for Passenger Car: The Ammonia-Storage Issue on a Fe-ZSM5 Catalyst." SAE International. doi:10.4271/2009-01-1929.

González-velasco, Juan R, Juan A Botas, Raquel Ferret, M Pilar González-marcos, Jean-louis Marc, and Miguel A Gutiérrez-ortiz. 2000. "Thermal Aging of Pd / Pt / Rh Automotive Catalysts under a Cycled Oxidizing - Reducing Environment” 59: 395-402.

Grossale, Antonio, Isabella Nova, and Enrico Tronconi. 2008. "Study of a Fe-Zeolite-Based System as NH3-SCR Catalyst for Diesel Exhaust Aftertreatment." Catalysis Today 136 (1-2): 18-27. doi:10.1016/j.cattod.2007.10.117.

Guralp, Orgun, Gongshin Qi, Wei Li, and Paul Najt. 2011. "Experimental Study of NO X Reduction by Passive Ammonia-SCR for Stoichiometric SIDI Engines." Society of Automotive Engineers (SAE). SAE International. doi:10.4271/2011-01-0307.

Haga, Hisao, Hiroyuki Kojima, Naoko Fukushi, Naoki Ohya, and Takuya Mito. 2015. "Optimized NH3 Storage Control for Next Generation Urea-SCR System.” SAE International. doi:10.4271/201501-1024. 
Herman, Andrew, Ming-Cheng Wu, David Cabush, and Mark Shost. 2009. "Model Based Control of SCR Dosing and OBD Strategies with Feedback from NH3 Sensors." SAE International. doi:10.4271/2009-01-0911.

Hsieh, M.-F., and J Wang. 2011. "Sliding-Mode Observer for Urea-Selective Catalytic Reduction (SCR) Mid-Catalyst Ammonia Concentration Estimation." International Journal of Automotive Technology 12 (3): 321. doi:10.1007/s12239-011-0038-4.

Hsieh, Ming Feng, and Junmin Wang. 2010. “An Extended Kalman Filter for Ammonia Coverage Ratio and Capacity Estimations in the Application of Diesel Engine SCR Control and Onboard Diagnosis." In Proceedings of the 2010 American Control Conference, 5874-79. doi:10.1109/ACC.2010.5530516.

Hsieh, Ming Feng, and Junmin Wang. 2011. "Development and Experimental Studies of a ControlOriented SCR Model for a Two-Catalyst Urea-SCR System.” Control Engineering Practice 19 (4). Elsevier: 409-22. doi:10.1016/j.conengprac.2011.01.004.

Huai, T, T D Durbin, J W Miller, J T Pisano, C G Sauer, S H Rhee, and J M Norbeck. 2003. "Investigation of NH3 Emissions from New Technology Vehicles as a Function of Vehicle Operating Conditions." Environ Sci Technol 37 (21): 4841-47.

I. Nova, M Colombo and E Tronconi. 2011. "Kinetic Modeling of Dynamic Aspects of the Standard NH3-SCR Reaction Over V2O5-WO3_TiO2 and Fe-Zeolite Commercial Catalysts for the Aftertreatment of Diesel Engines Exhausts." Oil \& Gas Science and Technology 66 (4): 681-91. doi:10.2516/ogst/2011132.

IARC, International Agency for Research on Cancer. 2013. "IARC: Outdoor Air Pollution a Leading Environmental Cause of Cancer Deaths." World Health Organization.

Jääskeläinen, Hannu, and W Addy Majewski. 2016. "Urea Dosing Control," 1-20.

Jinbiao, Ning, and Yan Fengjun. 2015. "Nonlinear Disturbance Observer Design for Estimation of Ammonia Storage Ratio in Selected Catalytic Reduction Systems.” In 2015 American Control Conference (ACC), 495-500. doi:10.1109/ACC.2015.7170784.

Johnson, Timothy V. 2009. "Diesel Emission Control in Review.” In SAE. Vol. 2012-01-03.

Kamasamudram, Krishna, Neal Currier, Tamas Szailer, and Aleksey Yezerets. 2010. "Why Cu- and FeZeolite SCR Catalysts Behave Differently At Low Temperatures." SAE International. doi:10.4271/2010-01-1182.

Karavalakis, Georgios, Maryam Hajbabaei, Yu Jiang, Jiacheng Yang, Kent C. Johnson, David R. Cocker, and Thomas D. Durbin. 2016. "Regulated, Greenhouse Gas, and Particulate Emissions from LeanBurn and Stoichiometric Natural Gas Heavy-Duty Vehicles on Different Fuel Compositions." Fuel 175. Elsevier Ltd: 146-56. doi:10.1016/j.fuel.2016.02.034.

Keuper, Andreas, Hiie-Mai Ida Unger, Jia Huang, Harald Bressler, and Wolfgang Albrecht. 2011. "Investigations to Achieve Highest Efficiencies in Exhaust Gas After-Treatment for Commercial Vehicles Using an SCR System.” SAE 2011-01-22.

Kim, Chang Hwan, Kevin Perry, Michael Viola, Wei Li, and Kushal Narayanaswamy. 2011. "Three-Way Catalyst Design for Urealess Passive Ammonia SCR: Lean-Burn SIDI Aftertreatment System." SAE Technical Paper. SAE International. doi:10.4271/2011-01-0306.

Koebel, Manfred, Martin Elsener, and Giuseppe Madia. 2001. "Recent Advances in the Development 
of Urea-SCR for Automotive Applications." SAE 2001-01-36.

Kröcher, O. 2007. "Chapter 9 Aspects of Catalyst Development for Mobile Urea-SCR Systems - From Vanadia-Titania Catalysts to Metal-Exchanged Zeolites." Studies in Surface Science and Catalysis 171 (January). Elsevier: 261-89. doi:10.1016/S0167-2991(07)80210-2.

Krupa, S V. 2003. "Effects of Atmospheric Ammonia (NH3) on Terrestrial Vegetation: A Review." Environ Pollut 124 (2): 179-221.

Li, Wei, Kevin L. Perry, Kushal Narayanaswamy, Chang Hwan Kim, and Paul Najt. 2010. "Passive Ammonia SCR System for Lean-Burn SIDI Engines." SAE International Journal of Fuels and Lubricants 3 (1). SAE International: 2010-01-0366. doi:10.4271/2010-01-0366.

Livingston, Cody, Paul Rieger, and Arthur Winer. 2009. "Ammonia Emissions from a Representative in-Use Fleet of Light and Medium-Duty Vehicles in the California South Coast Air Basin." Atmospheric Environment 43 (21): 3326-33. doi:10.1016/j.atmosenv.2009.04.009.

Majewski, W Addy. 2005. "Selective Catalytic Reduction." https://www.dieselnet.com/tech/cat_scr.php.

Marins, J. L. 2000. “An Extended Kalman Filter for Quaternion-Based Attitude Estimation.” Thesis Master, no. x: 3033-38.

http:/ oai.dtic.mil/oai/oai?verb=getRecord\&amp;metadataPrefix =html\&amp;identifier=ADA3 84973.

Matam, Santhosh Kumar, E H Otal, M H Aguirre, A Winkler, A Ulrich, D Rentsch, A Weidenkaff, and D Ferri. 2012. "Thermal and Chemical Aging of Model Three-Way Catalyst Pd/Al2O3 and Its Impact on the Conversion of CNG Vehicle Exhaust." Catalysis Today 184 (1): 237-44. doi:http://dx.doi.org/10.1016/j.cattod.2011.09.030.

Mathworks. 2016. "Fuzzy Logic Toolbox TM User Guide,” 1-285.

Mathworks. 2017. "Neural Network Toolbox User's Guide.”

MathWorks. 2017. "Solve Stiff Differential Equations and DAEs; Variable Order Method - MATLAB ode15s." Accessed November 22. http://www.mathworks.com/help/matlab/ref/ode15s.html.

McKinley, Thomas L, and Andrew G Alleyne. 2009. "A Switched, Controls-Oriented SCR Catalyst Model Using On-Line Eigenvalue Estimation.” SAE International. doi:10.4271/2009-01-1284.

Mejía-Centeno, Isidro, and Gustavo A. Fuentes. 2009. "Nitrous Oxide Formation during Light-off over a Commercial Pd-Containing Three-Way Catalytic Converter: The Effect of Low-Sulfur Gasoline." Chemical Engineering Communications 196 (10): 1140-51. doi:10.1080/00986440902831664.

Moos, Ralf, Ralf Müller, Carsten Plog, Aleksandar Knezevic, Holger Leye, Eckard Irion, Tillmann Braun, Klaus-Jürgen Marquardt, and Klaus Binder. 2002. "Selective Ammonia Exhaust Gas Sensor for Automotive Applications." Sensors and Actuators B: Chemical 83 (1-3): 181-89. doi:http://dx.doi.org/10.1016/S0925-4005(01)01038-3.

Nagashima, K, G Zhang, T Hirota, and H Muraki. 2000. "The Effect of Aging Temperature on Catalyst Performance of Pt/Rh and Pd/Rh TWCs.” SAE International. doi:10.4271/2000-01-1954.

NGK. 2017. "Primary Uses Sample Measurements."

NGV Global. 2017. “Current Natural Gas Vehicle Statistics.” http://www.iangv.org/current-ngvstats/.

Nova Enrico, Isabella; Tronconi. 2014. Urea-SCR Technology for deNOx After Treatment of Diesel 
Exhausts. Fundamental and Applied Catalysis. Springer: Springer New York.

Ohtsuka, Hirofumi. 2015. "Pt-Rh/CeO2-Al2O3 for Controlling Emissions from Natural Gas Engines: Three-Way Catalytic Activity at Low Temperatures and Effects of SO2 Aging." Emission Control Science and Technology 1 (1): 108-16. doi:10.1007/s40825-014-0009-0.

Ong, Chun, Anuradha Annaswamy, Ilya V Kolmanovsky, Paul Laing, and Dennis Reed. 2010. “An Adaptive Proportional Integral Control of a Urea Selective Catalytic Reduction System Based on System Identification Models." SAE International. doi:10.4271/2010-01-1174.

Pant, Atul, and Steven J. Schmieg. 2011. "Kinetic Model of NOx SCR Using Urea on Commercial CuZeolite Catalyst." Industrial and Engineering Chemistry Research 50 (9): 5490-98. doi:10.1021/ie200060s.

Perhinschi, Mario. 2016. Artificial Intelligence Techniques in MAE Chapter 3 Part 1,2,3 and 4.

Prié, Yannick, B E N Abdallah, B E N Abdallah, Mark Hudson Beale Martin T. Hagan Howard B. Demuth, Etudes Technologiques, Bacha Amira Date, Craig Warren, et al. 2015. "Neural Network Toolbox User â€TM S Guide.” MathWorks, no. June: 2-3. doi:10.1002/0471221546.

Prikhodko, Jim Parks and Vitaly. 2009. "Ammonia Production and Utilization in a Hybrid LNT+SCR System.” SAE Techinical Paper 4970 (November 2009): 2009-2739. doi:10.4271/2009-01-2739.

Prikhodko, Vitaly Y., James E. Parks, Josh A. Pihl, and Todd J. Toops. 2016. "Ammonia Generation and Utilization in a Passive SCR (TWC+SCR) System on Lean Gasoline Engine." SAE International Journal of Engines 9 (2). SAE International: 2016-01-0934. doi:10.4271/2016-010934 .

Quiros, David C, Arvind Thiruvengadam, Saroj Pradhan, Marc Besch, Pragalath Thiruvengadam, Berk Demirgok, Daniel Carder, Adewale Oshinuga, Tao Huai, and Shaohua Hu. 2016. "Real-World Emissions from Modern Heavy-Duty Diesel, Natural Gas, and Hybrid Diesel Trucks Operating Along Major California Freight Corridors." Emission Control Science and Technology 2 (3): 15672. doi:10.1007/s40825-016-0044-0.

Renner, Eberhard, and Ralf Wolke. 2008. "Formation of Secondary Inorganic Aerosols by High Ammonia Emissions Simulated by LM/MUSCAT.” In Air Pollution Modeling and Its Application XIX, edited by Carlos Borrego and Ana Isabel Miranda, 522-29. Dordrecht: Springer Netherlands. doi:10.1007/978-1-4020-8453-9_57.

Sabatini, Stefano, Irfan Kil, Travis Hamilton, Jeff Wuttke, Luis Del Rio, Michael Smith, Zoran Filipi, Mark A Hoffman, and Simona Onori. 2016. "Characterization of Aging Effect on Three-Way Catalyst Oxygen Storage Dynamics.” SAE International. doi:10.4271/2016-01-0971.

Sanchonx. 2017. "Interferometer for FTIR.” Accessed November 28. https://commons.wikimedia.org/wiki/File:FTIR_Interferometer.png.

Shi, Xian, Reinhard Seiser, Jyh-Yuan Chen, Robert Dibble, and Robert Cattolica. 2015. "Fuel-Dithering Optimization of Efficiency of TWC on Natural Gas IC Engine." SAE International. doi:10.4271/2015-01-1043.

Skaf, Zakwan, Timur Aliyev, Leo Shead, and Thomas Steffen. 2014. "The State of the Art in Selective Catalytic Reduction Control.” SAE International. doi:10.4271/2014-01-1533.

Song, Xiaobo. 2013. "A SCR Model Based on Reactor and Engine Experimental Studies for a Cu-Zeolite Catalyst.” Mechanical Engineering. Michigan Technological University.

Song, Xiaobo, Gordon Parker, John Johnson, Jeffrey Naber, and Josh Pihl. 2013. “A Modeling Study of 
SCR Reaction Kinetics from Reactor Experiments.” Sae Technincal Paper Series, no. 2013-011576. doi:10.4271/2013-01-1576.

Stanton, Donald, Stephen Charlton, and Phani Vajapeyazula. 2013. "Diesel Engine Technologies Enabling Powertrain Optimization to Meet U.S. Greenhouse Gas Emissions.” SAE International. doi:10.4271/2013-24-0094.

Stanton, Donald W. 2013. "Systematic Development of Highly Efficient and Clean Engines to Meet Future Commercial Vehicle Greenhouse Gas Regulations.” SAE International. doi:10.4271/201301-2421.

Suarez-Bertoa, R, A A Zardini, and C Astorga. 2014. "Ammonia Exhaust Emissions from Spark Ignition Vehicles over the New European Driving Cycle." Atmospheric Environment 97: 43-53. doi:http://dx.doi.org/10.1016/j.atmosenv.2014.07.050.

Sun, Kang, Lei Tao, David J Miller, Da Pan, Levi M Golston, Mark A Zondlo, Robert J Griffin, et al. 2016. "Vehicle Emissions as an Important Urban Ammonia Source in the United States and China." Environ Sci Technol. American Chemical Society. doi:10.1021/acs.est.6b02805.

Tengyu Liu Boguang Wang, Xiang Ding, Wei Deng, Sujun Lü, Yanli Zhang, Xinming Wang. 2014. "Emission Factor of Ammonia (NH3) from on-Road Vehicles in China: Tunnel Tests in Urban Guangzhou." Environment Research Letters 9 (6).

Tennison, Paul, Christine Lambert, and Michael Levin. 2004. "NOx Control Development with Urea SCR on a Diesel Passenger Car.” SAE International. doi:10.4271/2004-01-1291.

Theis, Joseph R., Jeong Kim, and Giovanni Cavataio. 2015. "Passive TWC+SCR Systems for Satisfying Tier 2, Bin 2 Emission Standards on Lean-Burn Gasoline Engines." SAE International Journal of Fuels and Lubricants 8 (2): 2015-01-1004. doi:10.4271/2015-01-1004.

Thiruvengadam, Arvind, Marc Besch, Daniel Carder, Adewale Oshinuga, Randall Pasek, Henry Hogo, and Mridul Gautam. 2016. "Unregulated Greenhouse Gas and Ammonia Emissions from Current Technology Heavy-Duty Vehicles." Journal of the Air \& Waste Management Association 66 (11): 1045-60. doi:10.1080/10962247.2016.1158751.

Upadhyay, Devesh, and Michiel Van Nieuwstadt. 2006. "Model Based Analysis and Control Design of a Urea-SCR deNOx Aftertreatment System.” Journal of Dynamic Systems, Measurement, and Control 128 (3): 737. doi:10.1115/1.2234494.

USEPA. 2011. “Department of Transportation.” Federal Register 76 (179). https://www.gpo.gov/fdsys/pkg/FR-2011-09-15/pdf/2011-20740.pdf.

USEPA. 2016. "Engine Certification and Compliance Testing." USEPA. https://www.epa.gov/vehicleand-fuel-emissions-testing/engine-certification-and-compliance-testing.

Wang, D, S Yao, D Racine, D Cabush, and M Shost. 2007. “Ammonia Sensor for SCR NOx Reduction.” In DEER Conf.

Wang, Dongliang, Kaisheng Huang, Xiaozhong Wu, and Yinhui Wang. 2011. "The Impact of Oxygen Sensor Degradation on Air-Fuel Ratio and Emissions." 2011 International Conference on Electric Information and Control Engineering, ICEICE 2011 - Proceedings, no. 2007: 2773-76. doi:10.1109/ICEICE.2011.5776926.

Willems, Frank, Robert Cloudt, Edwin van den Eijnden, Marcel van Genderen, Ruud Verbeek, Bram de Jager, Wiebe Boomsma, and Ignace van den Heuvel. 2007. "Is Closed-Loop SCR Control Required to Meet Future Emission Targets?” SAE Technical Papers 2007 (x). doi:10.4271/2007-01-1574. 
Zhang, Hui, Junmin Wang, and Yue Yun Wang. 2015. "Removal of NO Xsensor Ammonia Cross Sensitivity from Contaminated Measurements in Diesel-Engine Selective Catalytic Reduction Systems.” Fuel 150 (x): 448-56. doi:10.1016/j.fuel.2015.02.053.

Zhao, Yanguang, Jing Hu, Lun Hua, Shijin Shuai, and Jianxin Wang. 2011. "Ammonia Storage and Slip in a Urea Selective Catalytic Reduction Catalyst under Steady and Transient Conditions." Industrial \& Engineering Chemistry Research 50 (21). American Chemical Society: 11863-71. doi:10.1021/ie201045w. 


\section{APPENDIX A FTIR Specification}

\section{Table 16. FTIR Specification from Manufacturer}

\begin{tabular}{|c|c|}
\hline \multicolumn{2}{|l|}{ Analyzer } \\
\hline Model & MultiGas ${ }^{\text {TM }} 2030-\mathrm{HS}$ \\
\hline Measurement Technique & FTIR Spectrometry \\
\hline Gases and Vapors Measurable & Most molecules except for $\mathrm{N}_{2}, \mathrm{H}_{2}$, and $\mathrm{O}_{2}$ \\
\hline Ranges & Concentration setting between $100 \mathrm{ppb}$ and $100 \%$ full scale \\
\hline FTIR & 2102 Process FTIR \\
\hline Spectral Resolution & $0.5-128 \mathrm{~cm}^{-1}$ \\
\hline Scan Speed & 5 scans $/ \mathrm{sec} @ 0.5 \mathrm{~cm}^{-1}$ \\
\hline Scan Time & $0.2 \mathrm{sec}$ or longer \\
\hline Infrared Source & Silicon Carbide@ $1200^{\circ} \mathrm{C}$ \\
\hline Reference Laser & Helium Neon $\left(15798.2 \mathrm{~cm}^{-1}\right)$ \\
\hline Detector & $\mathrm{LN}_{2}$-cooled MCT; TE -cooled MCT \\
\hline \begin{tabular}{|l|} 
Purge Pressure \\
\end{tabular} & 20 psig (1.5 bar) max. \\
\hline Spectrometer Purge Flow & $0.2 \mathrm{~L} / \mathrm{min}$ of dry nitrogen or $\mathrm{CO}_{2}$ free clean dry air with dewpoints below $-70^{\circ} \mathrm{C}$ \\
\hline Optics Purge Flow & $0.2 \mathrm{~L} / \mathrm{min}$ of dry nitrogen or $\mathrm{CO}_{2}$ free clean dry air with dewpoints below $-70^{\circ} \mathrm{C}$ \\
\hline Pressure Transducer & MKS Baratron ${ }^{\circledR}$ \\
\hline Purge Connection & Swagelok $\AA_{\text {quick connect }}$ \\
\hline Computer & High speed xeon ${ }^{\mathrm{TM}}$ processor supplied with analyzer \\
\hline Communications & RJ-45 cross-over Ethernet \\
\hline Output Options & $\mathrm{xML}$, analog, AK, others (please inquire) \\
\hline Dimensions & $17.5 " \mathrm{~W} \times 12.5 " \mathrm{H}$ x $25.5 \mathrm{D} \mathrm{D}$ \\
\hline Installation & 19" rack mount chassis \\
\hline Power & 120 or $240 \mathrm{VAC}, 50 / 60 \mathrm{~Hz}, 3 \mathrm{amps}$ \\
\hline Weight & 110 lbs. $(50 \mathrm{~kg})$ \\
\hline \multicolumn{2}{|l|}{ Sampling Parameters } \\
\hline Sample Temperature & Ambient to $200^{\circ} \mathrm{C}$ (calibration temperature dependant) \\
\hline Sample Flow & $1-100+\mathrm{L} / \mathrm{min}$ \\
\hline \multirow{2}{*}{ Sample Pressure } & $0.0-1.3 \mathrm{~atm}$ (calibration pressure dependant) \\
\hline & $0.95-1.05 \mathrm{~atm}$ (nominal) \\
\hline \multicolumn{2}{|l|}{ Gas Cell } \\
\hline Construction & Nickel coated Al; Welded 316 stainless steel optional \\
\hline Fittings & $1 / 2 "$ Swagelok $®$ \\
\hline Tubing & Heated $1 / 2 "$ stainless steel \\
\hline Mirrors & Nickel plated aluminum substrate, with rugged gold coating \\
\hline Windows & $\mathrm{KBr} ; \mathrm{CaF}_{2}$ (others available) \\
\hline O-rings & Viton ${ }^{\circledR}$ (others available) \\
\hline \multicolumn{2}{|l|}{ Detection Limits } \\
\hline \multicolumn{2}{|c|}{ Low-level detection limits for the 5.11 meter gas cell and a mercury-cadmium-telluride (MCT) detector at $0.5 \mathrm{~cm}^{-1}$ resolution for typical } \\
\hline Name (formula) & Lowest Detectable Limit with $20 / 20^{\mathrm{TM}}$ Cell and $0.2 \mathrm{sec}$ Measurement \\
\hline Ammonia $\left(\mathrm{NH}_{3}\right)$ & $0.5 \mathrm{ppm}$ \\
\hline Carbon Dioxide $\left(\mathrm{CO}_{2}\right)$ & $0.2 \mathrm{ppm}$ \\
\hline Carbon Monoxide (CO) & $1.0 \mathrm{ppm}$ \\
\hline Formaldehyde $\left(\mathrm{H}_{2} \mathrm{CO}\right)$ & $0.6 \mathrm{ppm}$ \\
\hline Methane $\left(\mathrm{CH}_{4}\right)$ & $1.0 \mathrm{ppm}$ \\
\hline Nitrogen Dioxide $\left(\mathrm{NO}_{2}\right)$ & $0.5 \mathrm{ppm}$ \\
\hline Nitric Oxide (NO) & $1.0 \mathrm{ppm}$ \\
\hline Sulfur Dioxide $\left(\mathrm{SO}_{2}\right)$ & $1.0 \mathrm{ppm}$ \\
\hline xylenes $\left(\mathrm{C}_{8} \mathrm{H}_{10}\right)$ & $1.0 \mathrm{ppm}$ \\
\hline
\end{tabular}




\section{APPENDIX B Fuzzy Logic Controller Design and Program Code}

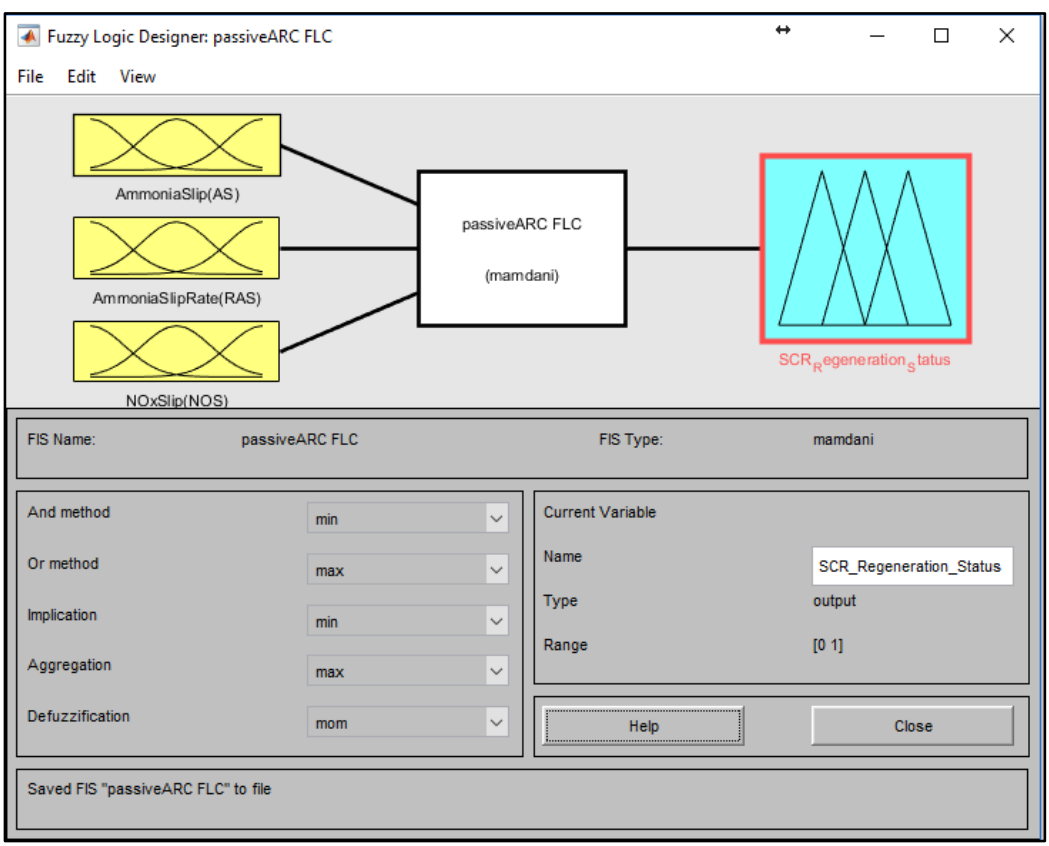

Figure 77. MATLAB ${ }^{\circledR}$ 's Fuzzy-Logic Toolbox (FL Main Design Interface)

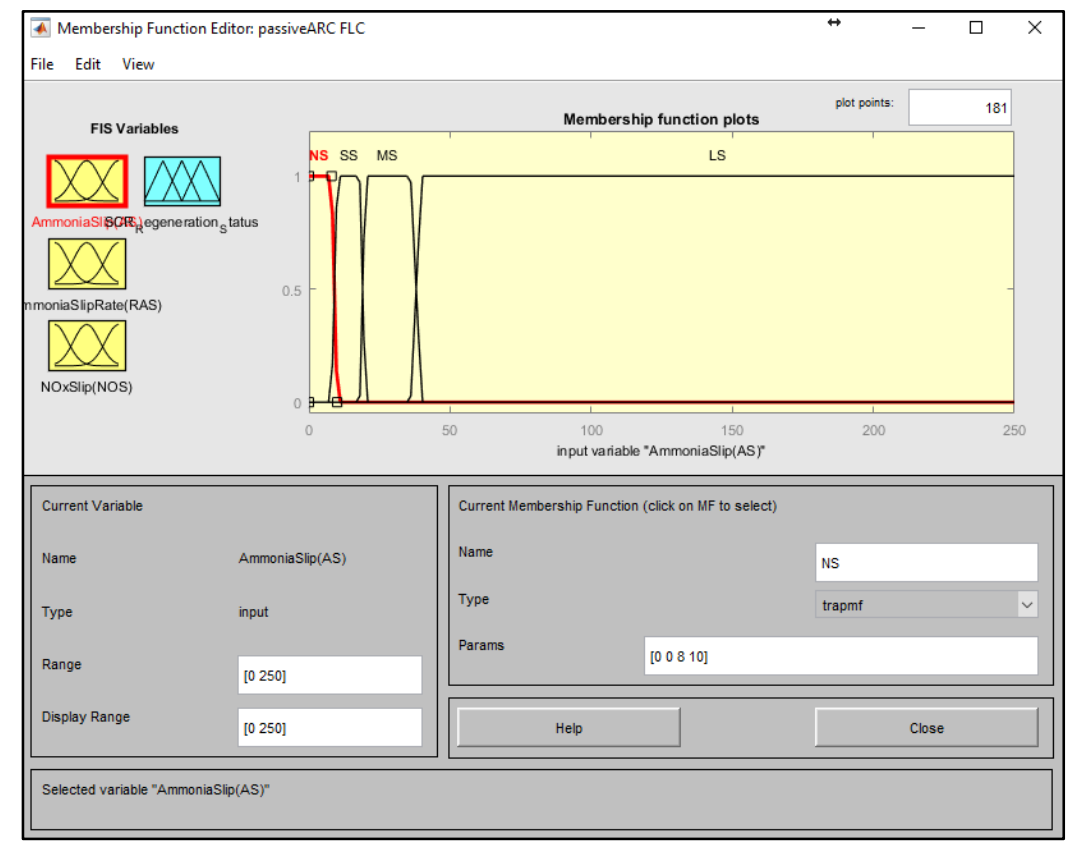

Figure 78. MATLAB ${ }^{\circledR}$ 's Fuzzy-Logic Toolbox (FL Membership Function Design Interface) 


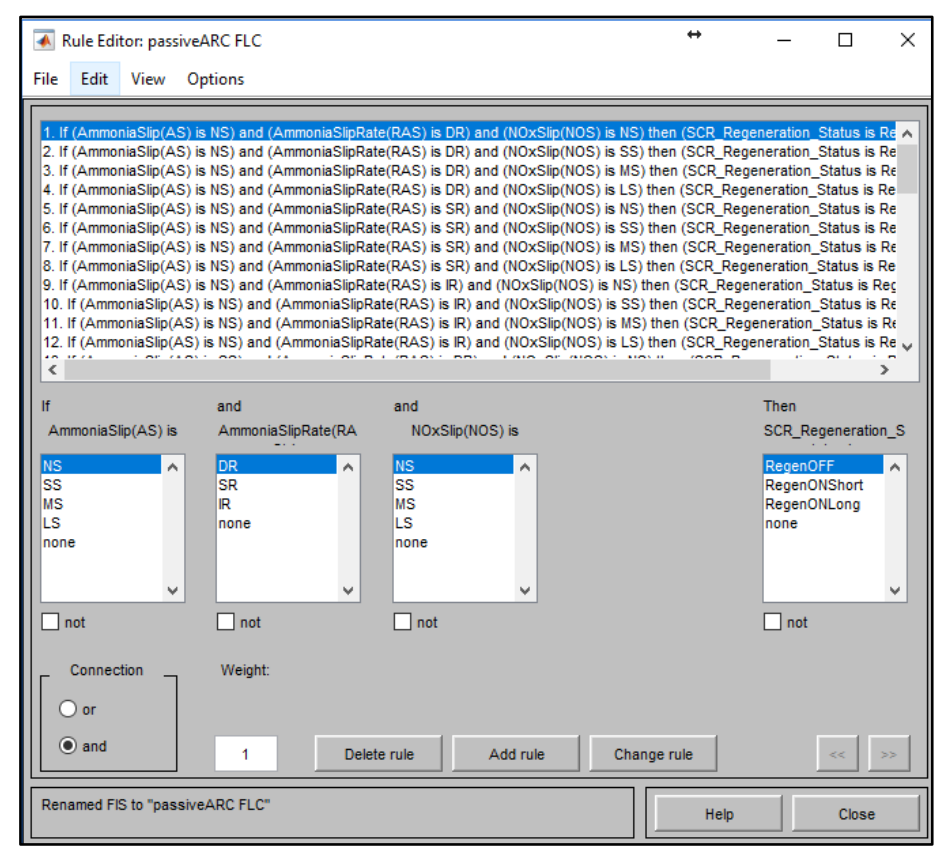

Figure 79. MATLAB ${ }^{\circledR}$ s Fuzzy-Logic Toolbox (FL Inference Rule Design Interface)

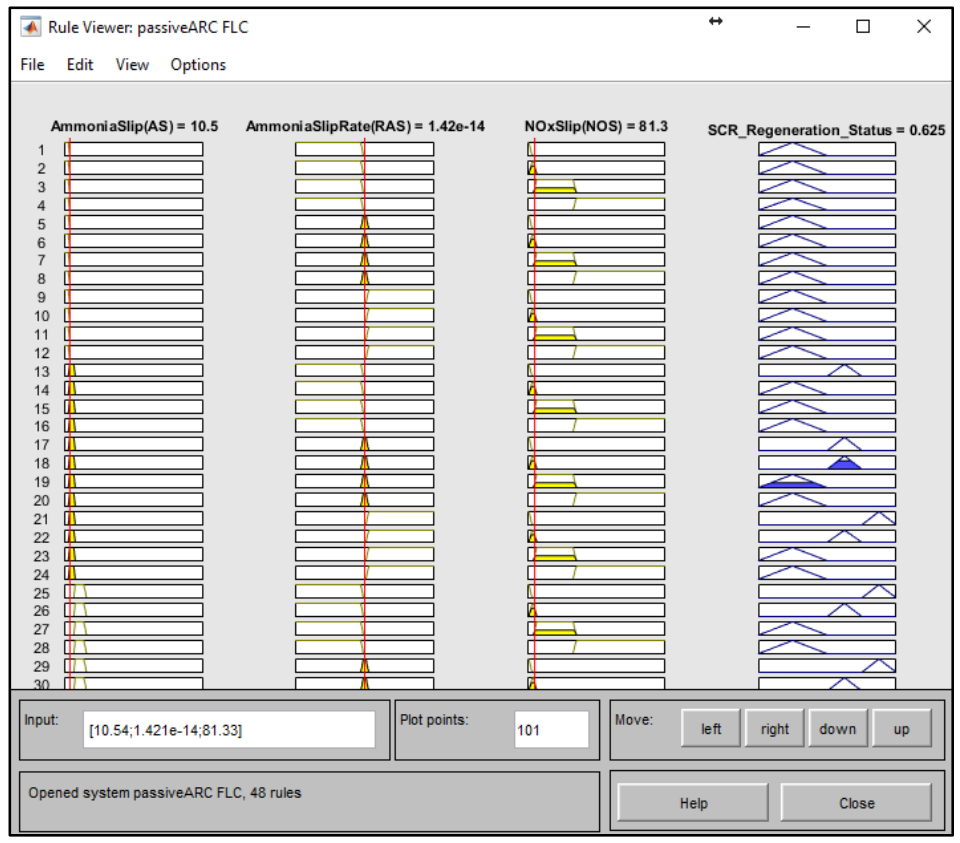

Figure 80. MATLAB ${ }^{\circledR}$ 's Fuzzy-Logic Toolbox (FL Rule Viewer) 
clc

clear all

o Import FL Controller: :

FLController = 'passiveARC FLC.fis';

$\mathrm{FLC}=$ readfis (FLName);

o Import Input Data: :

ExpData $\quad=$ 'FTP_1.mat';

NOS = Data.Sensor $\bar{N} O x(:, 1) ; \div$ [Units: ppm]

AS $=$ Data.SensorNH3 $(:, 1) ;$ 은 $[$ Units: ppm $]$

load (ExpData)

o Evaluate Inputs: :

\% Linguistic Variable-1 [Ammonia Slip, post SCR NH3] AS $($ AS $<0)=0$;

o Linguistic Variable-2 [Rate of Ammonia Slip]

RateDelay $=5$; $\%$ [Units: sec]

for $i=1$ :length (AS)

if $i>$ RateDelay

$\operatorname{RAS}(i)=$ AS (i) -AS (i-RateDelay);

else

RAS (i) $=0$; $\%$ [Units: ppm/period in secs]

end

end

o Linguistic Variable-3 [NOx slip]

$\operatorname{NOS}(\operatorname{NOS}<0)=0$;

\% Evaluate Output [Regeneration Status]

RegenStatus = evalfis $([A S, R A S, N O S], F L C)$;

FLC.fis

[System ]

name ='passiveARC FLC'

Type ='mamdani'

Version $\quad=2.0$

NumInputs $=3$

NumOutputs $=1$

NumRules $=48$

AndMethod ='min'

OrMethod ='max'

ImpMethod ='min'

AggMethod ='max'

DefuzzMethod=' mom'

[ Input 1]

Name ='Ammoniaslip (AS)' 


$$
\begin{aligned}
& \text { Range }=\left[\begin{array}{ll}
0 & 250
\end{array}\right] \\
& \text { NumMFs }=4 \\
& \text { MF1 ='NS': 'trapmf', [ }\left[\begin{array}{llll}
0 & 0 & 8 & 10
\end{array}\right] \\
& \text { MF2 ='SS':'trapmf', [ [ } \left.\begin{array}{llll}
8 & 10 & 18 & 20
\end{array}\right] \\
& \text { MF3 ='MS':'trapmf', [18 } 20 \text { 36 40 }] \\
& \text { MF4 ='LS':'trapmf', [ [ [ } \left.\begin{array}{llll}
36 & 40 & 250 & 500
\end{array}\right]
\end{aligned}
$$

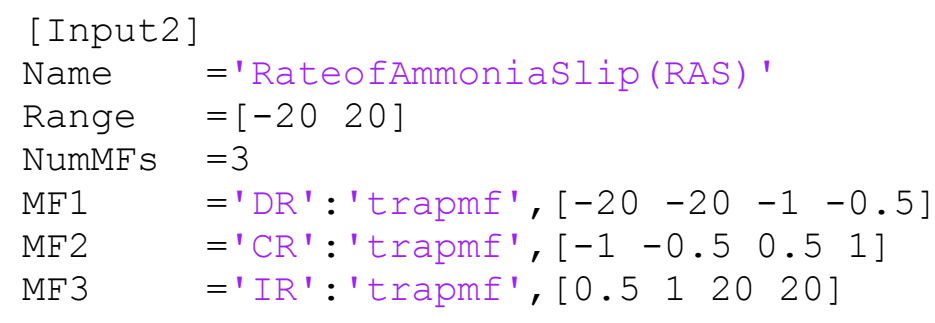

\section{[ Input 3]}

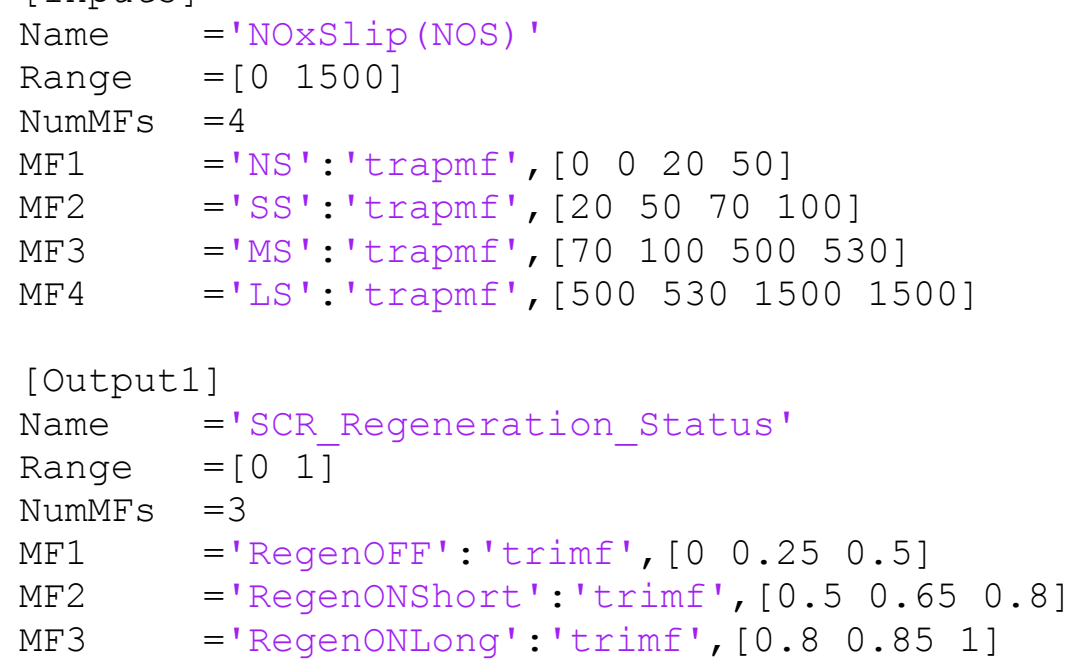

[Rules]

$\begin{array}{lllllll}1 & 1 & 1, & 1 & (1) & : & 1 \\ 1 & 1 & 2, & 1 & (1) & : & 1 \\ 1 & 1 & 3, & 1 & (1) & : & 1 \\ 1 & 1 & 4, & 1 & (1) & : & 1 \\ 1 & 2 & 1, & 1 & (1) & : & 1 \\ 1 & 2 & 2, & 1 & (1) & : & 1 \\ 1 & 2 & 3, & 1 & (1) & : & 1 \\ 1 & 2 & 4, & 1 & (1) & : & 1 \\ 1 & 3 & 1, & 1 & (1) & : & 1 \\ 1 & 3 & 2, & 1 & (1) & : & 1 \\ 1 & 3 & 3, & 1 & (1) & : & 1 \\ 1 & 3 & 4, & 1 & (1) & : & 1 \\ 2 & 1 & 1, & 2 & (1) & : & 1 \\ 2 & 1 & 2, & 1 & (1) & : & 1 \\ 2 & 1 & 3, & 1 & (1) & : & 1 \\ 2 & 1 & 4, & 1 & (1) & : & 1 \\ 2 & 2 & 1, & 2 & (1) & : & 1 \\ 2 & 2 & 2, & 2 & (1) & : & 1 \\ 2 & 2 & 3, & 1 & (1) & : & 1\end{array}$




$\begin{array}{lllllll}2 & 2 & 4, & 1 & (1) & : & 1 \\ 2 & 3 & 1, & 3 & (1) & : & 1 \\ 2 & 3 & 2, & 2 & (1) & : & 1 \\ 2 & 3 & 3, & 1 & (1) & : & 1 \\ 2 & 3 & 4, & 1 & (1) & : & 1 \\ 3 & 1 & 1, & 3 & (1) & : & 1 \\ 3 & 1 & 2, & 2 & (1) & : & 1 \\ 3 & 1 & 3, & 1 & (1) & : & 1 \\ 3 & 1 & 4, & 1 & (1) & : & 1 \\ 3 & 2 & 1, & 3 & (1) & : & 1 \\ 3 & 2 & 2, & 2 & (1) & : & 1 \\ 3 & 2 & 3, & 1 & (1) & : & 1 \\ 3 & 2 & 4, & 1 & (1) & : & 1 \\ 3 & 3 & 1, & 3 & (1) & : & 1 \\ 3 & 3 & 2, & 3 & (1) & : & 1 \\ 3 & 3 & 3, & 1 & (1) & : & 1 \\ 3 & 3 & 4, & 1 & (1) & : & 1 \\ 4 & 1 & 1, & 3 & (1) & : & 1 \\ 4 & 1 & 2, & 2 & (1) & : & 1 \\ 4 & 1 & 3, & 1 & (1) & : & 1 \\ 4 & 1 & 4, & 1 & (1) & : & 1 \\ 4 & 2 & 1, & 3 & (1) & : & 1 \\ 4 & 2 & 2, & 2 & (1) & : & 1 \\ 4 & 2 & 3, & 1 & (1) & : & 1 \\ 4 & 2 & 4 & 1 & (1) & : & 1 \\ 4 & 3 & 1, & 3 & (1) & : & 1 \\ 4 & 3 & 2, & 3 & (1) & : & 1 \\ 4 & 3 & 3, & 1 & (1) & : & 1 \\ 4 & 3 & 4 & 1 & (1) & : & 1\end{array}$

